Pacific Northwest

National Laboratory

Operated by Battelle for the

U.S. Department of Energy

\section{Predicting Peak Hydrogen Concentrations from Spontaneous Gas Releases in Hanford Waste Tanks}

\author{
CW Stewart \\ SA Hartley \\ PA Meyer \\ BE Wells
}

June 2005

Prepared for the U.S. Department of Energy under Contract DE-AC05-76RL01830 


\section{DISCLAIMER}

This report was prepared as an account of work sponsored by an agency of the United States Government. Neither the United States Government nor any agency thereof, nor Battelle Memorial Institute, nor any of their employees, makes any warranty, express or implied, or assumes any legal liability or responsibility for the accuracy, completeness, or usefulness of any information, apparatus, product, or process disclosed, or represents that its use would not infringe privately owned rights. Reference herein to any specific commercial product, process, or service by trade name, trademark, manufacturer, or otherwise does not necessarily constitute or imply its endorsement, recommendation, or favoring by the United States Government or any agency thereof, or Battelle Memorial Institute. The views and opinions of authors expressed herein do not necessarily state or reflect those of the United States Government or any agency thereof.

\section{PACIFIC NORTHWEST NATIONAL LABORATORY}

operated by

BATTELLE

for the

UNITED STATES DEPARTMENT OF ENERGY

under Contract DE-AC05-76RL01830

Printed in the United States of America

Available to DOE and DOE contractors from the

Office of Scientific and Technical Information,

P.O. Box 62, Oak Ridge, TN 37831-0062;

ph: (865) 576-8401

fax: (865) 576-5728

email: reports@adonis.osti.gov

Available to the public from the National Technical Information Service, U.S. Department of Commerce, 5285 Port Royal Rd., Springfield, VA 22161 ph: (800) 553-6847 fax: (703) 605-6900

email: orders@ntis.fedworld.gov

online ordering: http://www.ntis.gov/ordering.htm

This document was printed on recycled paper.

$$
06 / 05
$$




\section{Predicting Peak Hydrogen Concentrations from Spontaneous Gas Releases in Hanford Waste Tanks}

CW Stewart

SA Hartley

PA Meyer

BE Wells

June 2005

Pacific Northwest National Laboratory

Richland, WA 99352 


\begin{abstract}
Buoyant displacement gas release events (BDGRE) are spontaneous gas releases that occur in a few of the Hanford radioactive waste storage tanks when gas accumulation makes the sediment layer buoyant with respect to the liquid. BDGREs are assumed to be likely if the ratio of the predicted sediment gas fraction and neutral buoyancy gas fraction, or buoyancy ratio, exceeds unity. Based on the observation that the buoyancy ratio is also an empirical indicator of BDGRE size, a new methodology is derived that formally correlates the buoyancy ratio and the peak headspace hydrogen concentration resulting from BDGREs. The available data on the six historic BDGRE tanks, AN-103, AN-104, AN-105, AW-101, SY-103, and SY-101, are studied in detail to describe both the waste state and the corresponding distribution of BDGREs. The range of applicability of the buoyancy ratio-based models is assessed based on the modeling assumptions and availability of tank data. Recommendations are given for extending the range of the models' applicability.
\end{abstract}




\section{Summary}

Buoyant displacement gas release events (BDGRE) are spontaneous gas releases that occur in a few of the Hanford radioactive waste storage tanks with deep layers of liquid overlying a settled solids or sediment layer. BDGREs occur when sufficient gas generated by radioactivity and chemical reactions accumulates to make the sediment layer buoyant with respect to the liquid. BDGREs are the primary spontaneous flammable gas release hazard at Hanford, and methods have been developed over the last decade to classify waste tanks on their propensity for BDGREs.

The BDGRE criterion is based on the ratio of the average retained gas volume fraction in the sediment predicted for steady-state conditions and the gas volume fraction at neutral buoyancy, which is a function of the liquid and sediment densities. If the predicted gas fraction exceeds that required for neutral buoyancy, a BDGRE is possible. In this condition, the ratio of the predicted gas fraction and neutral buoyancy gas fraction, or buoyancy ratio, exceeds unity. Therefore, the primary safety criterion applied to tank farm operations is to maintain a buoyancy ratio of less than one.

However, it has been recognized for some time that the actual hydrogen concentrations induced by BDGREs in tanks with buoyancy ratios slightly greater than one are inconsequential. Tanks that have experienced more significant peak hydrogen concentrations during BDGREs have much higher buoyancy ratios. The buoyancy ratio can, in fact, serve as an empirical indicator of BDGRE size.

This report describes the derivation of a new methodology that formally correlates the buoyancy ratio and the peak headspace hydrogen concentration resulting from BDGREs. This flammability model can relate the buoyancy ratio criterion to a limiting headspace hydrogen concentration.

To provide a sound basis for the flammability model, the current understanding of gas retention and buoyant displacement gas release process is described and the history of its development reviewed. The flammability model relates tank behavior to tank conditions, both of which are described by a variety of data and observations. The available data on the six historic BDGRE tanks were studied in great detail to provide the best possible description of their waste state and the distribution of BDGREs that the tanks produced in that state.

The six tanks on which the flammability model is based are AN-103, AN-104, AN-105, AW-101, SY-103, and SY-101. Usable data describing BDGREs for these tanks begin in 19941995 and extend until 2002, when headspace gas monitoring was discontinued. SY-101 is the exception, having well-quantified BDGREs from December 1989 to July 1993, when BDGREs were stopped by operation of a jet mixer pump. Ventilation rates were increased in the three AN tanks and AW-101 in 1996. This additional cooling reduced their average sediment temperature 
and apparently the size of their BDGREs. Thus, the data for these tanks are divided into a "hot" period up through 1996 and a "cold" period from 1997 to the present, producing effectively ten "tanks" with identifiably different conditions and corresponding behaviors.

The variability in the BDGRE sizes and the uncertainty in the tank data were included in deriving the model through a Monte Carlo simulation. Uncertainty distributions were developed for each of the important variables used in calculating the buoyancy ratio, and the BDGRE history for each tank was fit with a distribution from which the $95^{\text {th }}$ percentile value was extracted to represent the maximum expected gas release. A function of the buoyancy ratio involving the headspace volume and hydrogen fraction of the retained gas was fit to the $95^{\text {th }}$ percentile BDGREs using a simple quadratic model. The buoyancy ratio criterion was then derived from the upper bound of the $95 \%$ confidence limit for the model fit.

The result relates the expected peak headspace hydrogen concentration in a tank to the calculated buoyancy ratio, tank headspace volume, and fraction of hydrogen in the retained gas. For example, the model would set a limiting buoyancy ratio of 2.6 for a tank with a headspace of $1,000 \mathrm{~m}^{3}$ and $50 \mathrm{vol} \%$ hydrogen fraction to prevent a BDGRE from creating flammable conditions in the tank headspace.

A detailed study of the range of applicability of the flammability model and of the previous buoyancy ratio criterion for preventing BDGREs was also performed. The major assumptions used in deriving the buoyancy ratio-based models were based on data from the five BDGRE tanks. Those with significantly stronger sediments were found to require some adjustment to inputs for calculating the buoyancy ratio, and brittle materials were determined to be outside the technical basis for the models in all respects.

It was also noted that, other than the five BDGRE tanks, there is little information available to describe the properties of the sediments (e.g., strong, brittle, etc.) as they now exist in the double-shell tanks or to anticipate the properties that might result from transfers or other waste management operations. Data on sediment properties and gas release behavior of the BDGRE tanks are also becoming outdated. Resumption of gas monitoring and in situ property measurements are recommended in selected tanks.

The enabling assumption for the flammability model is the acknowledgment that the BDGREs now occurring in five tanks are not hazardous. This realization allows specification of a limiting buoyancy ratio that is greater than one, allowing for the occurrence of inconsequential BDGREs but preventing the truly hazardous events. It provides a better definition of the safety margin of tanks that exhibit, or are predicted to exhibit, these BDGREs. 


\section{Contents}

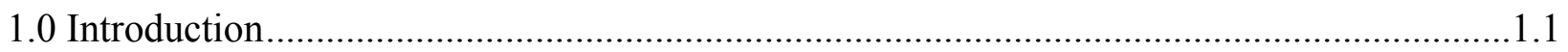

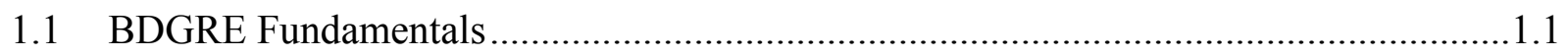

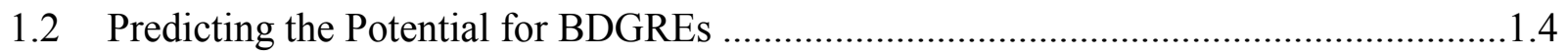

1.2.1 Early Observations, Insights, and Models ..............................................................

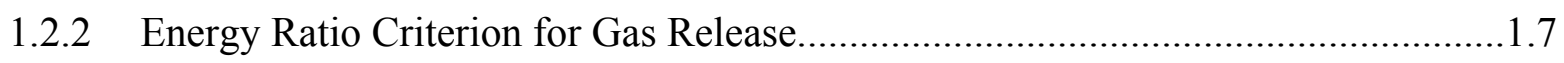

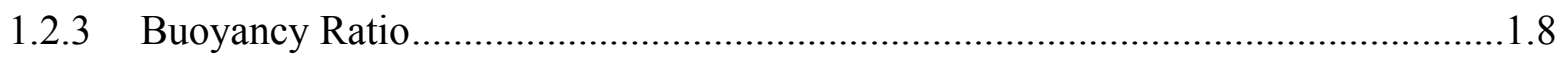

1.3 Application to Current Safety Authorization Basis...................................................1.11

1.3.1 Development of Flammable Gas Evaluation Methods ………..............................1.12

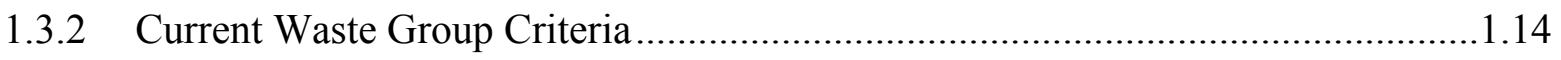

1.3.3 Problems in Applying the Waste Group Criteria .................................................1.15

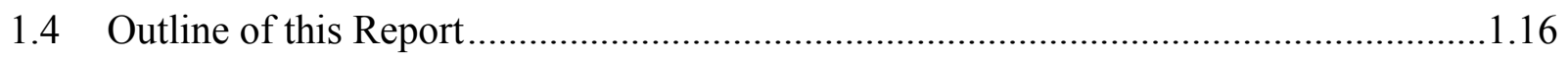

2.0 Data Sources for Waste Conditions and Behavior...............................................................

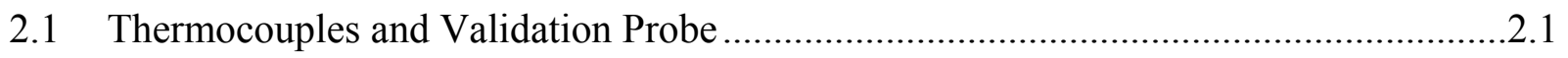

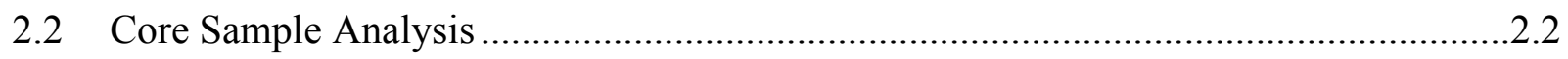

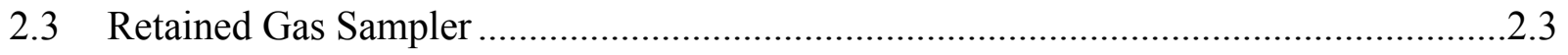

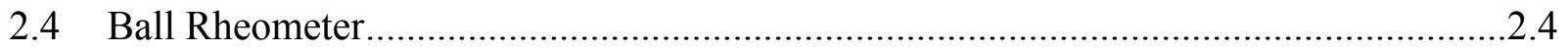

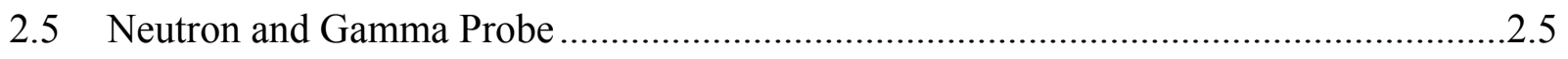

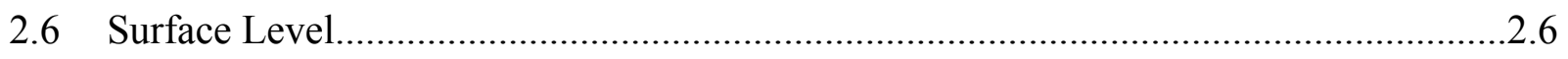

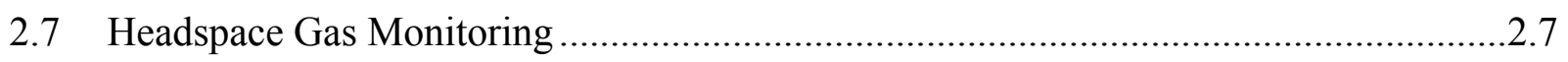

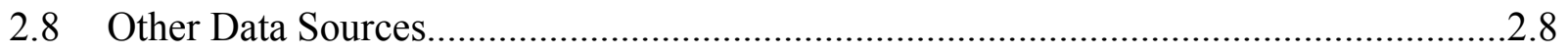

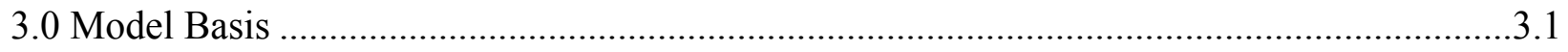

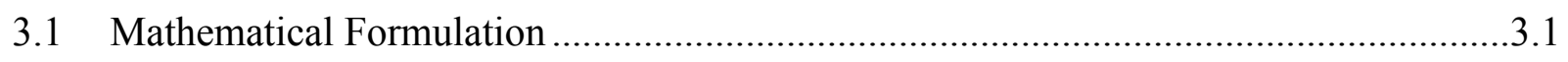

3.1.1 Tank Gas Release Data; Hot and Cold Periods .....................................................

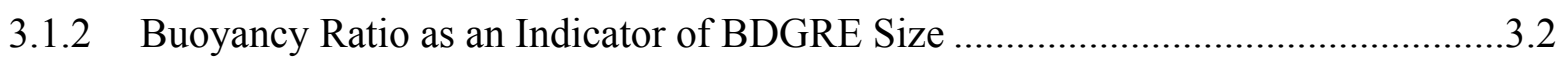

3.1.3 Physical Model Based on Rayleigh-Taylor Instability .............................................

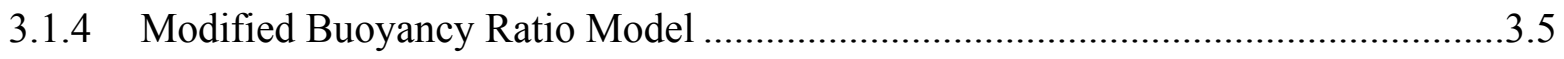

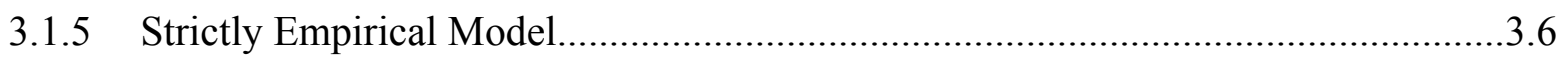

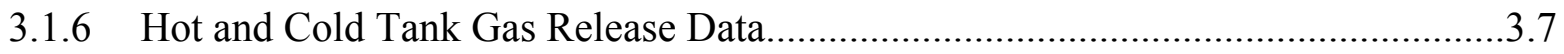

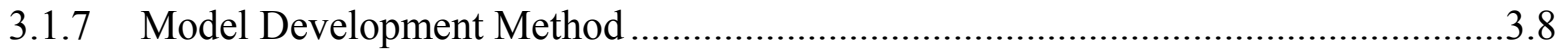

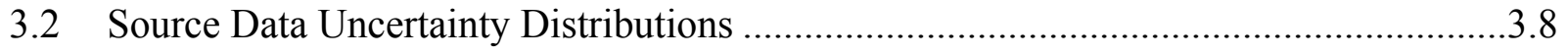

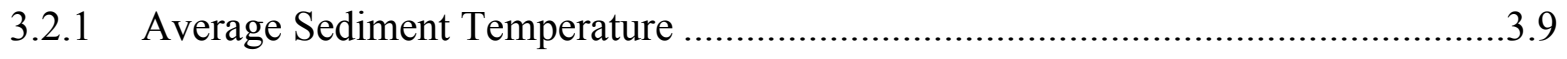

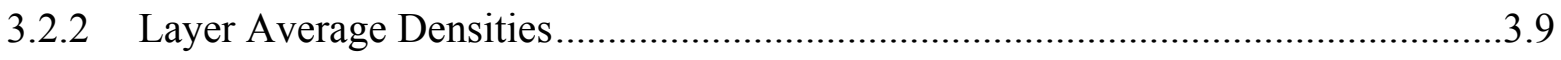

3.2.3 Waste Surface Level and Headspace Volume .........................................................23 


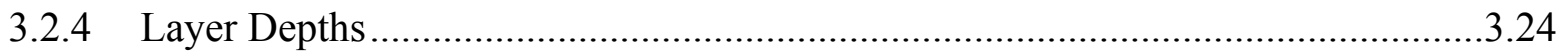

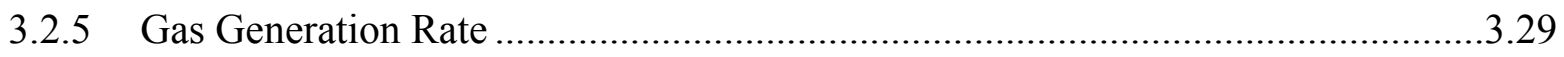

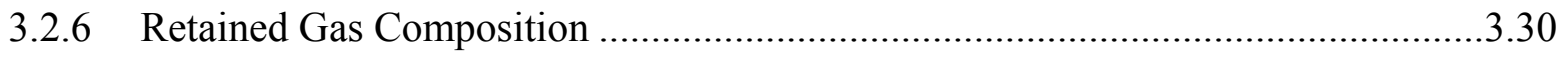

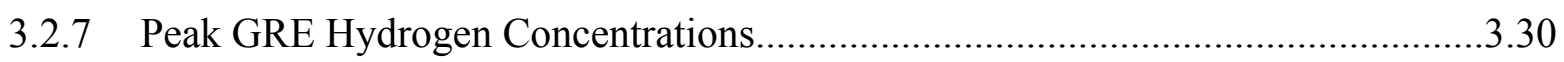

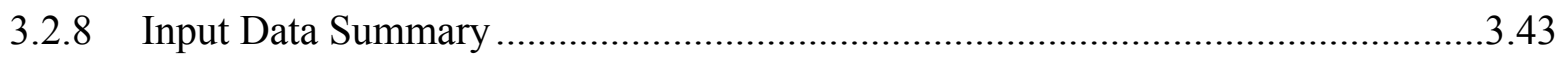

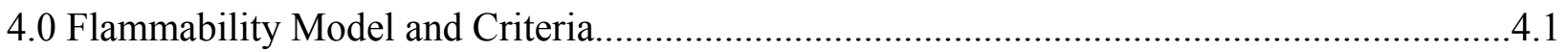

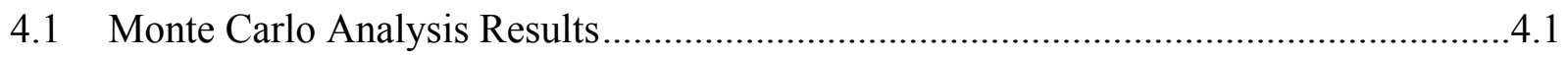

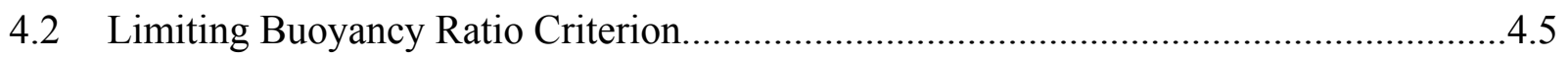

4.3 Recommendations for Applying the Flammability Model ............................................ 4.6

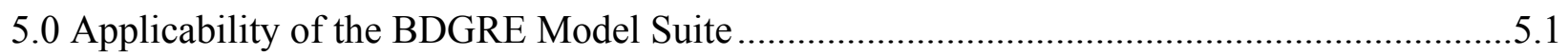

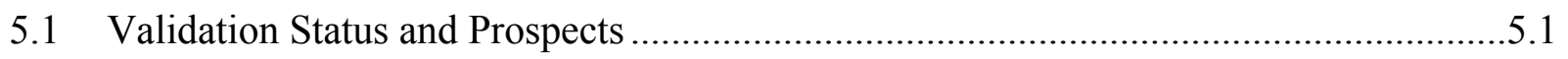

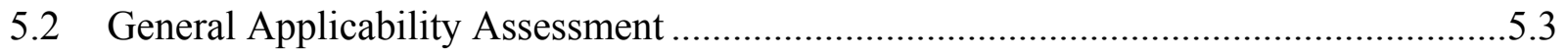

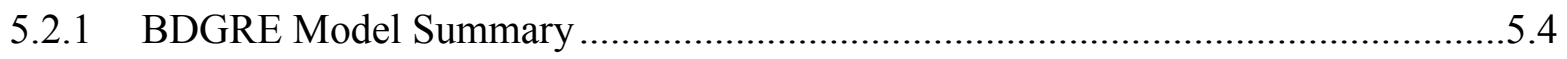

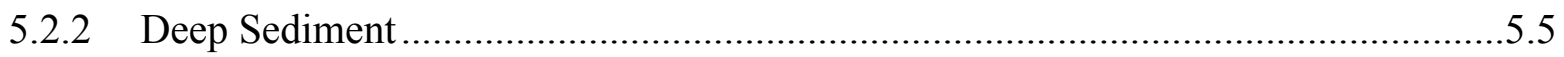

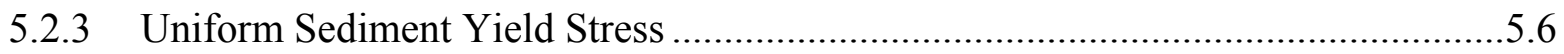

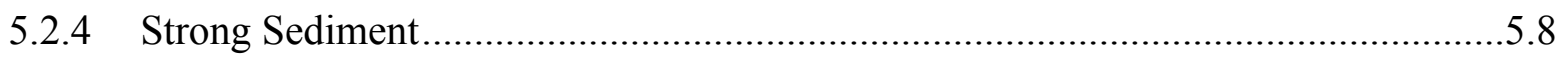

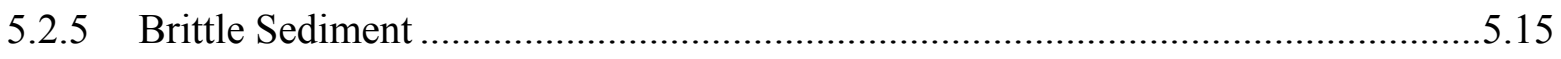

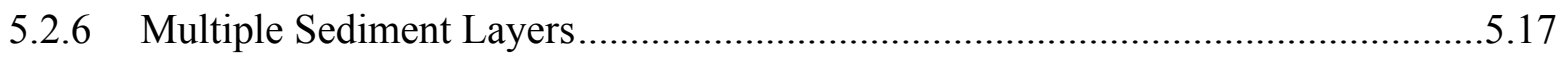

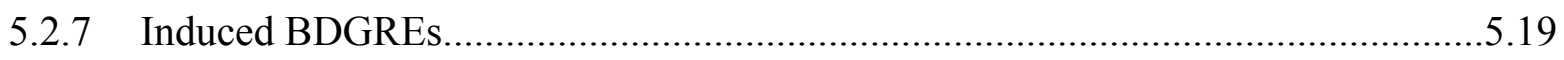

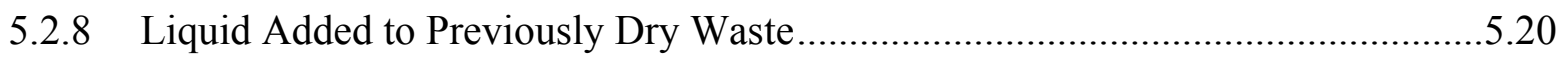

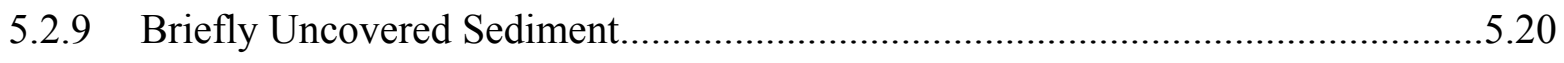

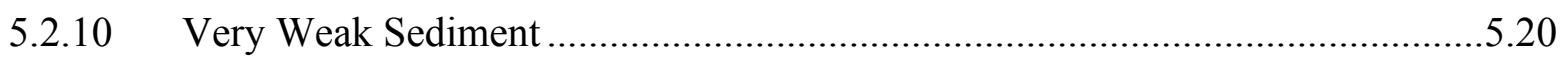

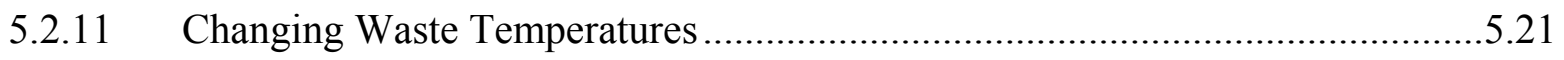

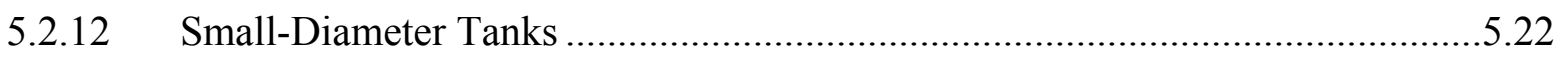

5.3 Determining Model Applicability from Waste Data.....................................................2.

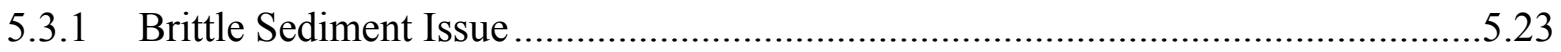

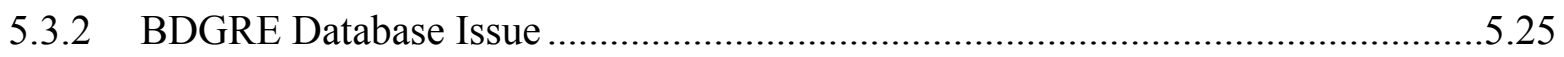

6.0 Conclusion and Recommendations on Model Applicability ................................................... 6.1

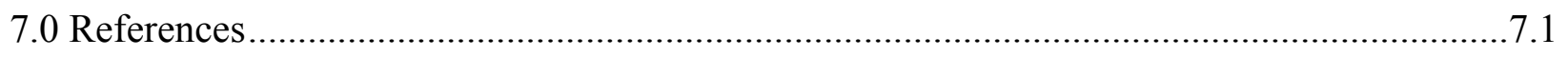

Appendix A - Gas Release from Buoyant Sediment Layers...................................................... 1

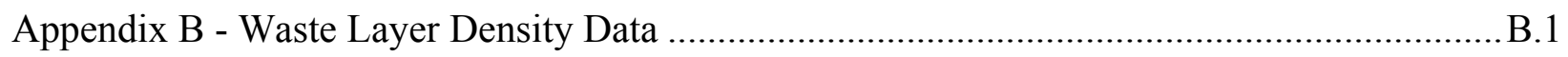

Appendix C - Cumulative Distribution Plots for Sediment Density Comparisons ...................... C.1

Appendix D - Combination, Uncertainty and Data for Sediment Layer Depth............................ 1

Appendix E - AN-107 Layer Density Consideration....................................................................

Appendix F - Statistical Model Methodology .................................................................... F.1 


\section{Figures}

1.1 Waste Configuration Susceptible to BDGREs ......................................................... 1.1

1.2 The Buoyant Displacement Gas Release Process .................................................... 1.2

3.1 Calculated Gas Release Volume Versus BR-1 ........................................................ 3.3

3.2 Measured Peak Hydrogen Concentration Versus (BR-1) $\left[\mathrm{H}_{2}\right]_{\text {gas }} / \mathrm{V}_{\mathrm{HS}}$.......................... 3.3

3.3 Performance of Rayleigh-Taylor Gas Release Model ............................................ 3.5

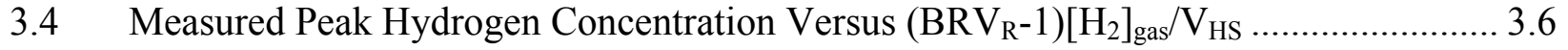

3.5 Measured Peak Hydrogen Concentration Versus $\left(\mathrm{V}_{\mathrm{R}}\right)_{\mathrm{E}}\left[\mathrm{H}_{2}\right]_{\text {gas }} / \mathrm{V}_{\mathrm{HS}}$........................ 3.7

3.6 Mass Fraction of Solids in Liquid Core Segments from AN-103 ............................. 3.13

3.7 Mass Fraction of Solids in Liquid Segments from AW-101 and SY-103 .................. 3.14

3.8 Liquid Density as a Function of Temperature ................................................. 3.17

3.9 Sediment Density in Sediment Core Segments from AN-103 ................................. 3.18

3.10 Sediment Density in Sediment Core Segments from AN-104.................................. 3.19

3.11 Sediment Density in Sediment Core Segments from AN-105 .................................. 3.19

3.12 Sediment Density in Sediment Core Segments from AW-101 ................................. 3.20

3.13 Example Neutral Buoyant Gas Fractions in AN-103 ................................................ 3.23

3.14 AN-103 Sediment-Liquid Interface Validation Probe Temperature Profile Example .. 3.26

3.15 Sediment Level Depth from Validation Probe Data as a Function of Time ................. 3.27

3.16 Crust Layer Thickness from Validation Probe Data as a Function of Time................. 3.27

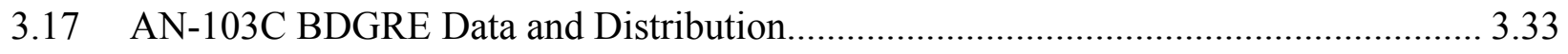

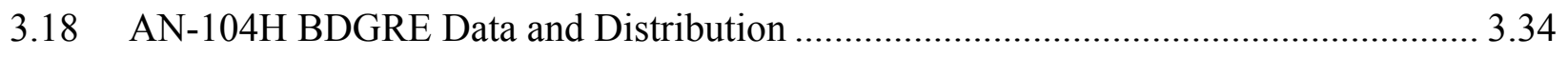

3.19 AN-104C BDGRE Data and Distribution......................................................... 3.34

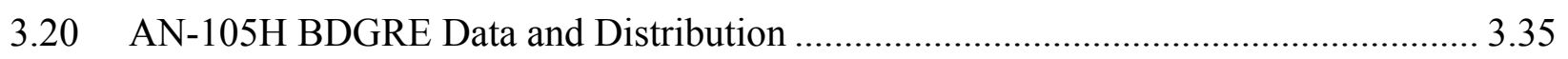

3.21 AN-105C BDGRE Data and Distribution............................................................ 3.35

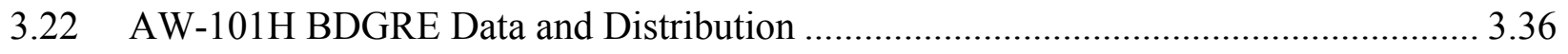

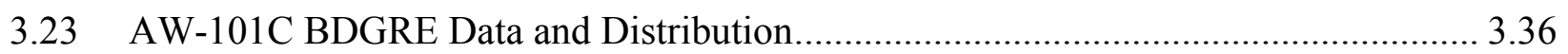

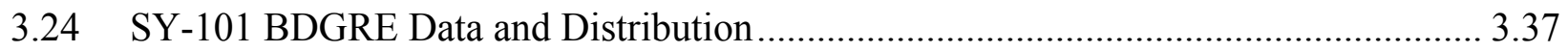

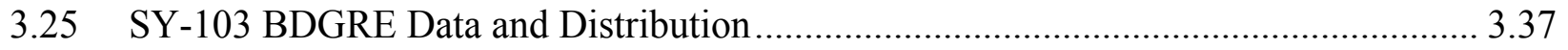

3.26 AN-103 Surface Level and GRE History, 1/95-1/00 …....................................... 3.38

3.27 AN-103 Surface Level and GRE History, 1/00-4/05 ….......................................... 3.39

3.28 AN-104 Surface Level and GRE History, 1/95-1/00 ............................................. 3.39

3.29 AN-104 Surface Level and GRE History, 1/00-4/05 …........................................ 3.40

3.30 AN-105 Surface Level and GRE History, 1/95-1/00 ............................................ 3.41

3.31 AN-105 Surface Level and GRE History, 1/00-4/05 …........................................ 3.41

3.32 AW-101 Surface Level and GRE History, 1/95-1/00 ............................................. 3.42 
3.33 AW-101 Surface Level and GRE History, 1/00-4/05 .......................................... 3.42

3.34 SY-103 Surface Level and GRE History, 1/95-1/00 ............................................. 3.43

3.35 SY-103 Surface Level and GRE History, 1/00-4/05 .............................................. 3.44

4.1 Cumulative Distributions of BR-1 ….............................................................. 4.2

$4.295^{\text {th }}$ Percentile BDGREs Compared to BDGRE Data ................................................ 4.3

4.3 Quadratic and Linear Models for $95^{\text {th }}$ Percentile Peak ................................................ 4.4

4.4 Range of Quadratic Models at Upper Bound of the 95\% Confidence Interval .............. 4.4

4.5 Limiting Buoyancy Ratio for $\left[\mathrm{H}_{2}\right]_{\text {peak }}$ of 40,000 ppm........................................... 4.6

5.1 Comparison of Gas Fraction Profiles.................................................................... 5.7

$5.2 \quad$ Bubble Shape and Retention Criteria Versus Yield Stress ...................................... 5.11

5.3 Gas Volume Fraction Profile in AN-103 .......................................................... 5.14

$5.4 \quad$ Stress-Strain Relationships for Bentonite Clay.................................................... 5.17 


\section{Tables}

3.1 Layer Density Results for AN-103 ............................................................ 3.11

3.2 Supernatant Liquid Density Distributions ............................................................. 3.17

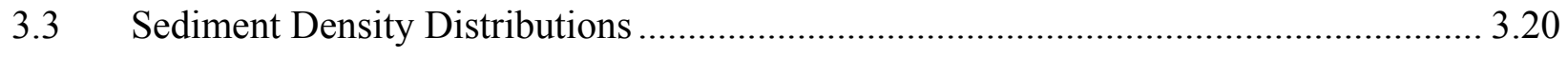

3.4 Comparison of Sediment Density Distributions ................................................... 3.23

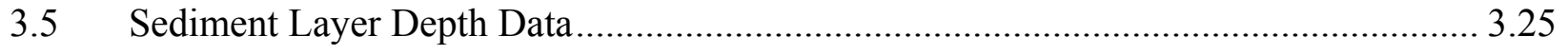

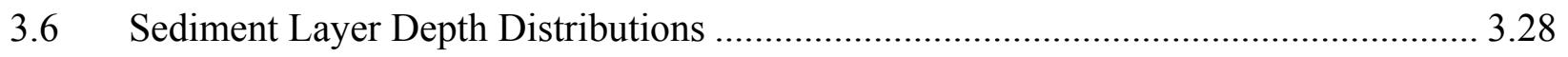

3.7 GRE Peak Hydrogen Concentrations.................................................................. 3.32

3.8 Peak Headspace Hydrogen Concentration Distributions ........................................... 3.33

3.9 Liquid Layer Density Uncertainty Distributions ............................................... 3.44

3.10 Sediment Layer Depth Uncertainty Distributions.................................................... 3.44

3.11 Hydrogen Generation Rate Uncertainty Distributions.............................................. 3.45

3.12 Hydrogen Fraction in the Retained Gas Uncertainty Distributions ............................ 3.45

3.13 Peak Headspace Hydrogen Concentration Distributions ........................................... 3.45

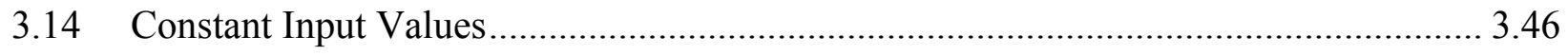

4.1 Statistics of BR-1 Output Distributions from the Monte Carlo Simulation................... 4.2

5.1 Evidence for Applying Buoyancy Ratio to Strong Sediment ................................... 5.12

5.2 Evidence for Applying the Flammability Model to Strong Sediment ....................... 5.15

5.3 Basis for Applying Buoyancy Ratio, Flammability and Energy Ratio Models............ 5.23 


\subsection{Introduction}

This report presents a method for predicting the potential flammability resulting from a specific kind of spontaneous gas release that occurs in a few of the Hanford radioactive waste storage tanks. These buoyant displacement gas release events (BDGRE) have been studied since the 1980s, when they became a concern in Tank SY-101. Since then, a considerable body of knowledge and several models have been developed that predict the conditions under which BDGREs may occur. These models have been incorporated into the tank farm safety authorization basis as described by Barker and Hedengren (2004).

The methodology presented in this report extends these models to better define the safety margin of tanks that exhibit, or are predicted to exhibit, these BDGREs. This section provides background information on the mechanisms operating during BDGREs, the history and results of modeling work, and how they are used in the current authorization basis.

\subsection{BDGRE Fundamentals}

The radioactive wastes in Hanford's underground tanks are a byproduct of plutonium production from 1945 to the late 1980s. The many processes involved in half a century of managing these wastes have formed a waste configuration in some tanks consisting of a layer of supernatant liquid overlying a layer of settled solids or sediment. Because the liquid is mixed by natural convection, it is called the convective layer. The strength of the sediment inhibits convection, so it is called the nonconvective layer. There may also be a floating layer of solids and trapped gas bubbles called the "crust." This waste configuration is sketched in Figure 1.1.

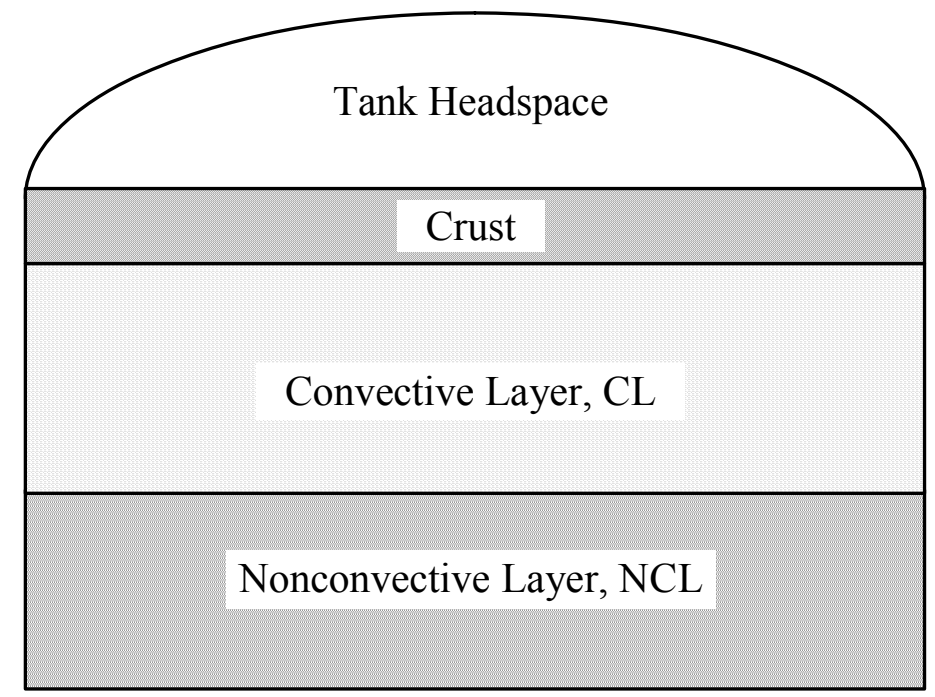

Figure 1.1. Waste Configuration Susceptible to BDGREs 
All radioactive wastes generate a flammable gas mixture, both by radiolysis of water and thermal and radiolytic decomposition of organic solvents consisting of hydrogen (fuel), nitrous oxide (oxidizer), and nitrogen (inert) and small amounts of ammonia, methane, and other hydrocarbons. These gases accumulate as bubbles that impart their buoyant force to the surrounding sediment as they grow. This is illustrated by object (1) in Figure 1.2. When the bubbles grow so that a portion, or "gob," of the sediment becomes sufficiently buoyant to overcome its weight and the strength of the surrounding material restraining it, the gob breaks away and rises through the liquid layer above it (object 2). The trapped gas bubbles expand as the gob rises, failing the surrounding material and allowing some of the bubbles to escape (object 3). The gob releases gas at the waste surface until it is no longer buoyant and sinks back to the sediment layer (object 4).

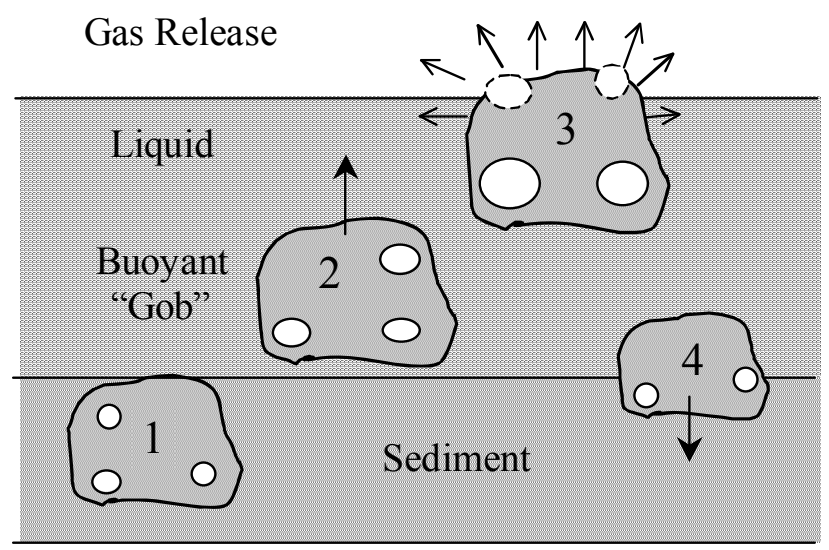

Figure 1.2. The Buoyant Displacement Gas Release Process

The retained gas volume required for buoyancy is determined by the ratio of the bulk densities of the convective and nonconvective layers. (a) The gas volume fraction or void fraction in the nonconvective layer at neutral buoyancy (bulk density of nonconvective layer equal to the convective layer density) is given by

$$
\alpha_{\mathrm{NB}}=1-\frac{\rho_{\mathrm{L}}}{\rho_{\mathrm{S}}}
$$

where $\rho_{\mathrm{L}}$ and $\rho_{\mathrm{S}}$ are the bulk degassed densities of the convective liquid and nonconvective sediment layers, respectively.

Individual gobs are believed to reach buoyancy and undergo buoyant displacement more or less independently, so different regions of the tank are in different stages of evolution toward the

(a) A BDGRE requires a slightly higher gas volume fraction than simple neutral buoyancy to counteract the restraining effect of waste strength. However, for typical 4-m-deep sediment with yield stress of $200 \mathrm{~Pa}$, the difference is only 0.4 vol\%. At 1,000 Pa the difference increases to 2 vol\% (see Appendix A). 
event with a timing that is almost random. The effect is similar to popcorn, with each grain heating up and popping independently of the rest. Variability in BDGRE size and period comes from both randomness of gas accumulation and the specific condition left by the preceding gob.

The larger gas releases require participation of multiple gobs. In SY-101, the in-tank video showed that large gas releases involved four or five gobs of various sizes. Though the basic model assumes the gobs behave independently, the buoyant displacement process obviously causes a relatively strong disturbance and temporarily suspends a large mass of solid particles in the supernatant liquid. This increases the effective density of the convective layer and reduces the neutral buoyancy gas fraction via Eq. (1.1). If another gob is nearly buoyant, the reduction in $\alpha_{N B}$ could be enough to break it free. A higher gas generation rate would bring more gobs closer to neutral buoyancy, making multiple gob releases by this mechanism more likely.

The hydrogen concentration in the tank headspace resulting from a BDGRE depends on the size of the release, the headspace volume, the fraction of hydrogen in the retained gas, and the average hydrostatic pressure from which the gas is released. The major portion of a BDGRE occurs rapidly relative to the headspace ventilation rate and can be considered instantaneous, though the entire process may last for several days. This "prompt release," which creates the peak hydrogen concentration, is the primary flammability hazard. (a) The peak headspace hydrogen concentration, $\left[\mathrm{H}_{2}\right]_{\text {peak }}(\mathrm{ppm})$, resulting from a prompt gas release of volume $\mathrm{V}_{\mathrm{R}}$ (at the headspace pressure) can be expressed as

$$
\left[\mathrm{H}_{2}\right]_{\text {peak }}=\frac{\mathrm{V}_{\mathrm{R}}\left[\mathrm{H}_{2}\right]_{\text {gas }} 10^{6}}{\mathrm{~V}_{\mathrm{HS}}}
$$

where $\mathrm{V}_{\mathrm{HS}}$ is the tank headspace volume and $\left[\mathrm{H}_{2}\right]_{\text {gas }}$ is the hydrogen mole fraction of the retained gas. Eq. (1.2) can be used to calculate the peak headspace hydrogen concentration resulting from a predicted gas release volume or to determine the prompt gas release volume corresponding to a measured headspace hydrogen concentration.

Following the model illustrated in Figure 1.2, the gas in the participating gob expands in proportion to the ratio of its in situ pressure to the headspace pressure and releases gas only until it becomes nonbuoyant at the surface. Thus the total gas release volume from a BDGRE, $V_{R, t o t a l}$, is approximately (see Appendix A):

$$
\mathrm{V}_{\mathrm{R}, \text { total }}=\alpha_{\mathrm{NB}} \mathrm{V}_{\text {gob }}\left(\frac{\mathrm{p}_{\text {gas }}}{\mathrm{p}_{\mathrm{HS}}}-1\right)
$$

(a) Other fuel gases, including ammonia, methane, and other hydrocarbons, also contribute to headspace flammability. However, hydrogen is dominant in BDGREs, and the error in neglecting these gases is smaller than uncertainties in the hydrogen fraction itself. 
where $\mathrm{V}_{\mathrm{gob}}$ is the total (in situ) volume of the participating gobs, $\mathrm{p}_{\mathrm{HS}}$ is the headspace pressure, and $p_{\text {gas }}$ is the average hydrostatic pressure at which the gas is held in the gob. Eq. (1.3) represents an upper bound to the prompt release volume.

Assuming that the gas bubbles support the bulk density of both solids and liquid in the nonconvective layer, the pressure is approximated by

$$
p_{\text {gas }}=p_{H S}+g\left[\left(H_{W}-H_{S}\right) \rho_{L}+\frac{H_{S}}{2} \rho_{S}\right]
$$

where HW is the waste surface level, and HA is the sediment layer depth. Alternatively, assuming the solid particles support themselves in lithostatic contact while the accumulating gas displaces liquid, the gas pressure is

$$
\mathrm{p}_{\text {gas }}=\mathrm{p}_{\mathrm{HS}}+\rho_{\mathrm{L}} \mathrm{g}\left(\mathrm{H}_{\mathrm{W}}-\frac{\mathrm{H}_{\mathrm{S}}}{2}\right)
$$

The few in situ pressure measurements within the nonconvective layer(a) support Eq. (1.5). Further, comparing the bulk densities of core samples with in situ retained gas measurements clearly shows that gas has displaced liquid.(b) However, the difference in calculated pressure between Eq. (1.4) and (1.5) is small for tanks of concern.

\subsection{Predicting the Potential for BDGREs}

A method to predict which tanks and what conditions could exhibit BDGREs is needed to ensure flammable gas safety for operation of the waste tanks. The search for such a method began almost as soon as the magnitude of the hazard was recognized in early 1990. A physically based model became available in 1997 (Meyer and Wells 2000) and was incorporated into the tank farm safety authorization basis in 2003 (Barker and Hedengren 2003).

\subsubsection{Early Observations, Insights, and Models}

Concern about BDGREs first involved the behavior of tank SY-101. Between April 1977 and November 1980, the tank was filled with double shell slurry, the most concentrated waste that the 242-A evaporator could produce, and complexed concentrate, a moderately concentrated waste rich in organic complexants that fuel gas generation. By the time the tank was filled, it

(a) The pressure sensors on the velocity-density-temperature trees (VDTT) installed in SY-101 in early 1993 provided data for several months before the VDTTs failed.

(b) The density profile in tanks AN-103 and AN-105, for example, closely follow the profile of gas volume fraction measured by the void fraction instrument (VFI) (Hedengren et al. 2000). 
had experienced a "slurry growth," a rise in surface level attributed to gas buildup, of 23 inches out of a total depth of 408 inches. In January 1981 the waste level inexplicably dropped, indicating SY-101's first BDGRE, though it was not recognized as such at the time. The waste level rose dropped three inches in March 1981, initiating a regular rise-drop cycle with a period of about three months.

The size of these level drops, which indicated the volume of gas released, kept increasing. On July 4, 1983 a large BDGRE pressurized the tank headspace for the first time. After that, headspace pressurization became a regular occurrence.(a) Concern over the apparently increasing size of gas releases in SY-101 precipitated a temporary gas monitoring effort that measured hydrogen concentrations above the lower flammability limit during a BDGRE on July $16,1985$.

This discovery called for immediate remedial action. Accordingly, the nonconvective layer was lanced periodically with water jets and later with air jets in attempts to release gas in a semicontrolled manner to prevent the largest "burps." Water lancing was discontinued in 1986 because it was slowly filling up the tank, and air lancing ceased in 1989 because it was also increasing the waste level without materially mitigating gas releases. However, the now known potential for a flammable headspace in SY-101 was not considered a critical safety hazard because the headspace was flammable for only a few hours at most and no viable ignition source was present.

A major policy change occurred in early 1990, when a technical reviewer noted that nitrous oxide was present at about the same concentration as hydrogen in SY-101's retained gas. Because nitrous oxide is an oxidizer, it was possible for the retained gas to burn without being released into the headspace. This theoretically put the retained gas at risk of deflagration continuously, not just for the few hours it was flammable in air after a release. When this hazard was announced to the public in March 1990, research on SY-101's gas releases began in earnest.

Beginning with the event of December 29, 1989, each BDGRE was analyzed to the extent that recorded data allowed. Initially these data comprised only waste level, waste temperature at several locations, headspace pressure, and ventilation flow rate. After the April 19 and August 5, 1990 gas release events, it became clear from comparisons of the calculated gas volume necessary to account for the measured headspace pressure rise and that associated with the measured surface level drop that the gas was coming from near the bottom of the tank. The sudden change in waste temperature profile from hot near the bottom to hot near the top corroborated this and clearly indicated that material was physically moving from bottom to top at the same time. This was, in fact, the very picture of a BDGRE, but it would be at least a year before these observations were connected with buoyancy.

(a) Induced ventilation maintains the tank headspace at a slight vacuum to force all released gas through exhaust HEPA filters. A gas release large enough to defeat the existing vacuum can cause an "unfiltered release" and automatically becomes a safety issue. 
In the meantime, on November 5, 1990, Public Law 101-510, Section 3137, "Safety Measures for Waste Tanks at Hanford Nuclear Reservation," was signed into law. Also known as the "Wyden Amendment" after its author, Senator Ron Wyden of Oregon, the law required identification of tanks with "a serious potential for release of high-level waste" due to uncontrolled increases in temperature or pressure, including flammable gas ignition. The list of tanks identified as having a flammable gas problem became known as the "Flammable Gas Watch List."

The original flammable gas watch list was published in February 1991. SY-101 was on the original list. The criteria for placing tanks on the watch list could be considered the first "BDGRE criteria." Flammable gas watch list tanks were those that exhibited:

- waste level increases without liquid addition

- unexplained headspace pressurization

- unexplained waste temperature changes

- a surface crust

- total organic carbon (TOC) concentrations above $3 \mathrm{~g} / \mathrm{L}$

- waste from B Plant (these wastes contained organic complexants).

The first three criteria were evidence of actual BDGREs while the last three might be considered "predictive" for tanks that may have the potential for BDGREs. These criteria relate mainly to gas generation. Waste with high organic carbon, particularly organic complexants, have potentially high gas generation rates. A surface crust would occur in a highly concentrated waste where the densities of the convective and nonconvective layers are similar and the neutral buoyancy gas fraction is low. From the discussion in Section 1.1, a tank with a high gas generation rate and low neutral buoyancy gas fraction would have a high potential for BDGREs.

Based on observations of the results of evaporator runs compared with the ensuing tank behavior, it was found that BDGREs occurred only in tanks that had been highly concentrated, i.e., those with high average specific gravity. In 1995, a criterion for waste transfers was set to limit the resulting average waste specific gravity to below 1.41 (Fowler 1995). While the average specific gravity represents the physics of buoyant displacements only in the broadest sense, it was easy to apply and separated BDGRE tanks from non-BDGRE tanks reasonably well.

Because the average specific gravity appeared to be a good indicator of BDGRE behavior, Estey and Guthrie (1996) looked at other variables and combinations of variables to see if a more precise empirical criterion could be developed. They found that the product of the convective layer specific gravity and the sediment depth was the best discriminator of those they evaluated. Based on the tank data at that time, a specific gravity-sediment depth product greater than 150 inches was the criterion for BDGRE tanks. 
A much more complicated set of criteria based on the concentrations of dissolved aluminum, sodium, nitrite, and TOC in the liquid were proposed (Slezak et al. 1998). These species were indirect indicators of the degree of concentration or evaporation (sodium and nitrite) and the gas generation rate (TOC, aluminum and nitrite). However, in practice they proved to be poor discriminators of BDGRE behavior and saw little use.

All of these early attempts at criteria for predicting BDGRE behavior were purely empirical and were related in only the most general way to the actual physics of the process. However, the physics of BDGREs were beginning to be described in more detail, and the accumulating body of knowledge and experience eventually led to the development of the buoyancy ratio model.

\subsubsection{Energy Ratio Criterion for Gas Release}

With the BDGRE process reasonably well defined for tanks with similarly deep convective and nonconvective layers, investigation began in 1996 into the possible gas release mechanisms in tanks with other waste configurations. Because BDGREs had been observed to be large and potentially hazardous, an obvious concern was whether they could happen in other tanks. Because the buoyancy of sediment with respect to an overlying liquid is a fundamental requirement of a BDGRE, the presence of both sediment and liquid layers is necessary. Also, because gas expansion in a rising gob is the primary gas release mechanism, the liquid depth must provide for sufficient expansion. A balance of the energy required to liquefy the material in the gob with the energy liberated by bubble expansion provided an expression for the liquid depth requirement (Stewart et al. 1996a).

The potential energy released by the buoyant gob $\mathrm{E}_{\mathrm{B}}$ rising a distance $\mathrm{L}$ from the surface to the sediment layer is calculated by integrating the net buoyant force exerted by the gob over this distance. Neglecting the small increase in gas volume fraction required for the gob to break free from the surrounding sediment, the integration results in

$$
\mathrm{E}_{\mathrm{B}}=\alpha_{\mathrm{NB}} \mathrm{V}_{\mathrm{gob}} \mathrm{p}_{\mathrm{HS}} \gamma[(1+1 / \gamma) \ln (1+\gamma)-1]
$$

where

$\mathrm{V}_{\text {gob }}=$ volume of the gob, including gas

$\mathrm{p}_{\mathrm{HS}}=$ ambient tank headspace pressure

$\gamma \quad=$ normalized change in hydrostatic pressure from the top of the sediment to the waste surface, $\gamma=\rho_{\mathrm{L}} \mathrm{g}\left(\mathrm{H}_{\mathrm{W}}-\mathrm{H}_{\mathrm{S}}\right) / \mathrm{p}_{\mathrm{HS}}$

With simplifying assumptions about the stress-strain relationship of the sediment, and assuming that liquefaction occurs at a strain of 1 (see subsection 4.2.2.3), the energy required to fluidize the gob is expressed as 


$$
\mathrm{E}_{\mathrm{L}}=\left(1-\alpha_{\mathrm{NB}}\right) \mathrm{V}_{\mathrm{gob}} \tau_{\mathrm{y}}
$$

where $\tau_{\mathrm{y}}$ is the sediment material yield stress. ${ }^{(a)}$

A gas release requires that the buoyant energy released by a gob, $E_{B}$, be greater than the energy required to yield the gob, $\mathrm{E}_{\mathrm{L}}$. That is, $\mathrm{E}_{\mathrm{B}} / \mathrm{E}_{\mathrm{L}}>1$. Forming the ratio from Eq. (1.6) and (1.7),

$$
\mathrm{ER}=\frac{\mathrm{E}_{\mathrm{B}}}{\mathrm{E}_{\mathrm{L}}}=\frac{\alpha_{\mathrm{NB}} \mathrm{p}_{\mathrm{HS}} \gamma}{\left(1-\alpha_{\mathrm{NB}}\right) \tau_{\mathrm{y}}}[(1+1 / \gamma) \ln (1+\gamma)-1]>1
$$

Realizing that some of the energy released is dissipated in turbulent flow around the rising gob, and in small-scale slurry flows within the disintegrating gob, the energy ratio required for gas release should be considerably greater than unity. A series of bench-top experiments was conducted with bentonite clay with gas generated by decomposition of hydrogen peroxide to quantify the required ratio (Stewart et al. 1996a). These tests showed that a vigorous gas release occurred at an energy ratio of 5 but little or no gas release at an energy ratio of 2.6. Based on these results, and the calculated energy ratios of Tank SY-101, a reasonable criterion for gas release from a BDGRE is ER $>3$. (b)

\subsubsection{Buoyancy Ratio}

During 1996 and 1997, waste sampling and characterization activities were greatly expanded and accelerated in response to Recommendation 93-5 of the Defense Nuclear Facilities Safety Board (DNFSB), which mandated a better understanding of tank behavior. This work employed the ball rheometer to measure the in situ waste rheology, the void fraction instrument (VFI) to measure the gas volume fractions, and the retained gas sampler to obtain the retained gas composition and volume fraction (Stewart et al. 1996b, Mahoney et al. 1999). At the same time, laboratory studies were performed on gas generation and gas retention using a wide variety of simulants as well as real waste samples. The enormous amount of new data from these measurements and experiments, added to the experience and monitoring data from the large BDGREs in SY-101 prior to 1993 and the expanded tank headspace gas monitoring campaign, provided a broad foundation from which to make a quantum leap in understanding of the gas retention and release processes in the waste tanks.

(a) "Yield stress" is used for yield stress in shear, commonly referred to as shear strength. The quantity may be defined as the point at which a material ceases to deform like a solid under applied stress but instead flows like a truly viscous material with a finite viscosity.

(b) Gas release attributable to other mechanisms besides a BDGRE was observed at lower energy ratios (Stewart et al. 1996a). 
Three key insights from these data were 1) the gas volume fraction generally increased downward from essentially zero at the top of the sediment and sometimes followed a parabolic profile with a maximum below the sediment midplane, 2) the sediment yield stress increased linearly downward from zero at the top of the sediment, and 3) the gas fraction profiles were often very dissimilar at different locations in the tank. The causes of all three observations were interrelated. At the same time it was obvious that, because gas is generated continuously and uniformly throughout the waste but only a few tanks retain enough gas to have BDGREs, there must be a slow, almost imperceptible bubble migration up through the sediment that releases the generated gas in most tanks before it becomes buoyant. The balance of gas generation and bubble migration determined the steady-state gas volume fraction in the sediment (Bohl 1997).

The mechanism for this background bubble release is not known, and it has not been observed in the laboratory. However, it must exist or all tanks (with sufficient supernatant liquid) would have buoyant displacements. To illustrate the phenomenon, assume a flux of gas occurs from the waste surface and apply the ideal gas law to mass conservation on the gas in the sediment layer assuming a constant density. The result is

$$
\frac{\mathrm{d} \alpha}{\mathrm{dt}}=\mathrm{G} \frac{\mathrm{RT}_{\mathrm{S}}}{\mathrm{p}_{\text {gas }}}-\frac{\mathrm{J}_{\alpha}}{\mathrm{H}_{\mathrm{S}}}
$$

where $\mathrm{G}$ is the average gas generation rate (moles $\left./ \mathrm{m}^{3}-\mathrm{s}\right), \mathrm{p}_{\text {gas }}$ and $\mathrm{T}_{\mathrm{S}}$ are the average gas pressure $(\mathrm{Pa})$ and temperature $(\mathrm{K})$, respectively, $\mathrm{R}$ is the gas constant $(8314 \mathrm{~J} / \mathrm{mole}-\mathrm{K}), \mathrm{H}_{\mathrm{S}}$ is the height of the sediment layer (m), and $\mathrm{J}_{\alpha}$ is the average gas volume flux across the sediment layer surface $\left(\mathrm{m}^{3} / \mathrm{m}^{2}-\mathrm{s}\right)$.

Eq. (1.9) illustrates the simple balance between source and outflow that determines whether the gas volume fraction in the sediment increases. If the volumetric gas generation exceeds the rate at which gas leaves the sediment layer, gas must accumulate. The gas volume flux can be expressed as the product of the number density, $n\left(1 / \mathrm{m}^{3}\right)$, volume, $v_{B}\left(\mathrm{~m}^{3}\right)$, and velocity, $\mathrm{u}_{B}$ $(\mathrm{m} / \mathrm{s})$, of bubbles crossing the upper surface of the sediment layer:

$$
\mathrm{J}_{\alpha}=\mathrm{nv}_{\mathrm{B}} \mathrm{u}_{\mathrm{B}}
$$

Substituting Eq. (1.10) into Eq. (1.9) and replacing the product of the bubble volume and number density with the volume gas volume fraction gives

$$
\frac{\mathrm{d} \alpha}{\mathrm{dt}}+\alpha \frac{\mathrm{u}_{\mathrm{B}}}{\mathrm{H}_{\mathrm{S}}}=\mathrm{G} \frac{\mathrm{RT}_{\mathrm{S}}}{\mathrm{p}_{\text {gas }}}
$$

In the steady state, the gas fraction in Eq. (1.11) reduces to 


$$
\alpha_{\mathrm{SS}}=\mathrm{G} \frac{\mathrm{RT}_{\mathrm{S}}}{\mathrm{p}_{\mathrm{gas}}} \frac{\mathrm{H}_{\mathrm{S}}}{\mathrm{u}_{\mathrm{B}}}
$$

Eq. (1.12) shows that the average sediment layer gas volume fraction at steady state depends on the average volumetric gas generation rate, the depth of the sediment layer, and the bubble migration velocity. The greater the gas generation rate is and the deeper the sediment, the higher the average gas volume fraction is. However, if the steady-state gas volume fraction is greater than the neutral buoyancy gas fraction (Eq. 1.1), a buoyant displacement will occur before the tank arrives at its steady state. Therefore, if the bubble rise velocity and gas generation rate were known, the potential for a buoyant displacement could be evaluated.

It was postulated that the unknown slow bubble migration mechanism followed the trend of Stokes' equation for creeping flow around a sphere. Stokes flow can be expressed as:

$$
u_{B}(z)=S \frac{\rho_{S} g v_{B}(z)^{2 / 3}}{\mu(z)}
$$

where $\mathrm{S}$ is a proportionality constant $(\mathrm{a})$ and $\mu$ is an effective bulk viscosity $(\mathrm{kg} / \mathrm{m}-\mathrm{s})$ that describes the retarding force on the bubble. The linear variation in the yield stress, $\tau_{\mathrm{y}}$, as determined from in situ measurements with the ball rheometer, can be used as a proportional indicator for the effective viscosity in Eq. (1.13). The resulting bubble migration velocity equation is

$$
\mathrm{u}_{\mathrm{B}}(\mathrm{z})=\frac{\mathrm{SK}}{\mathrm{m}_{\tau}} \frac{\rho_{\mathrm{S}} \mathrm{gv}_{\mathrm{B}}(\mathrm{z})^{2 / 3}}{\left(\mathrm{H}_{\mathrm{S}}-\mathrm{z}\right)}
$$

where $\mathrm{K}$ is the unknown proportionality constant between the unknown effective viscosity and the yield stress, and $\mathrm{m}_{\tau}$ is the slope of the yield stress versus depth curve representing the ball rheometer data (Meyer and Wells 2000).

The variation of the gas volume fraction with elevation, $\mathrm{z}$, in the steady state can be described by one-dimensional conservation equations for the bubble mass and number. These are expressed, respectively by

and

$$
\frac{\mathrm{d}\left(\mathrm{mu}_{\mathrm{B}}\right)}{\mathrm{dz}}=\mathrm{G}(\mathrm{z})
$$

(a) For true Stokes flow, $u_{B}=\left(\rho_{S} g d^{2}\right) / 18 \mu$ (Fan and Tsuchiya 1990) so that replacing $d$ with $v_{B}=\pi d^{2} / 6$ requires $\mathrm{S}=\frac{2}{9}\left(\frac{3}{4 \pi}\right)^{2 / 3}$ 


$$
\frac{\mathrm{d}\left(\mathrm{nu}_{\mathrm{B}}\right)}{\mathrm{dz}}=\mathrm{N}(\mathrm{z})
$$

where $\mathrm{m}$ is the number of moles of gas in bubbles per unit total volume, and $\mathrm{N}$ is the bubble nucleation rate per unit volume.

Combining Eq. (1.15) and (1.16), assuming the gas generation rate and bubble nucleation rate are uniform, defining the dimensionless elevation $\eta=\mathrm{z} / \mathrm{H}_{\mathrm{S}}$, and substituting $\mathrm{u}_{\mathrm{B}}$ from Eq. (1.14) gives the following equation for the gas volume fraction profile (Stewart and Meyer 2005):

$$
\alpha(\eta)=\frac{m_{\tau}}{S K \rho_{S} g} N^{2 / 3}\left(\frac{R T_{S}}{p_{\text {gas }}}\right)^{1 / 3} G^{1 / 3} H_{S}^{2} \eta(1-\eta)
$$

Eq. (1.17) describes a parabola with a maximum at $\eta=0.5$, similar to the gas volume fraction profiles measured with the VFI. The upper portion of a layer of sediment with this profile has a low gas volume fraction that must be balanced by the higher gas fractions below before the layer can be buoyant. This means that only the portion of the layer whose average gas volume fraction, computed down from the top, equals the neutral buoyancy gas fraction described by Eq. (1.1) will participate in a BDGRE. Performing this integration and dividing by the righthand side of Eq. (1.1) forms the buoyancy ratio, BR:

$$
\begin{aligned}
\mathrm{BR} & =\frac{\mathrm{C}}{\rho_{\mathrm{S}}-\rho_{\mathrm{L}}}\left(\frac{\mathrm{GT}_{\mathrm{S}}}{\mathrm{p}_{\text {gas }}}\right)^{1 / 3} \mathrm{H}_{\mathrm{S}}^{2} \\
\mathrm{C} & =\left\lfloor\frac{3}{16} \frac{\mathrm{N}^{2 / 3} \mathrm{R}^{1 / 3} \mathrm{~m}_{\tau}}{\mathrm{SKg}}\right\rfloor
\end{aligned}
$$

where

The coefficient $\mathrm{C}$ in Eq. (1.18) contains all the constants along with unknowns that must be determined empirically from tank data. To do this, the coefficient $\mathrm{C}$ is adjusted so that the minimum buoyancy ratio of the five tanks actually exhibiting BDGREs is exactly one. Thus, $\mathrm{BR} \geq 1$ indicates that a tank may have BDGREs, and $\mathrm{BR}<1$ indicates that a tank will not have BDGREs.

\subsection{Application to Current Safety Authorization Basis}

While the energy ratio and buoyancy ratio models were being developed, the body of tank data and experience generated in response to DNFSB Recommendation 93-5 was organized and documented for the double-shell tanks (Hedengren et al. 2000) and single-shell tanks (Hedengren 
et al. 2001) on the flammable gas watch list. These reports described the current understanding of gas generation, retention, and release mechanisms and provided data on the waste configuration, properties, retained gas volume and composition, and gas release history. The successful remediation of BDGREs by diluting the waste in Tank SY-101 also provided a case study in applying this body of knowledge (Johnson et al. 2000). These summary documents formed the basis for closure of the flammable gas safety issue (Johnson et al. 2001) and the removal of all tanks from the flammable gas watch list in 2001.

With the safety issue closed, attention turned to day-to-day tank farm operations, looking ahead to the eventual retrieval of waste from the single-shell tanks into the 28 double-shell tanks for staging into the future waste vitrification plant. The emphasis thus turned from evaluating the flammable gas hazard in existing tanks to controlling transfer, mixing, and concentrating operations to manage the available tank space safely. Methods were needed to evaluate the potential flammable gas hazards of an operation based on predicted waste conditions and to adjust or control the operation as necessary to avoid hazards.

\subsubsection{Development of Flammable Gas Evaluation Methods}

The grouping of tanks by flammable gas hazard is based on the volume of flammable gas a tank can hold and how readily it can be released by a waste disturbance or spontaneous release. These behaviors are evaluated based on the waste type, configuration, and properties. However, experience showed by the mid-1990s that the traditional safety analysis approach of using bounding values of each data item in a calculation could not be reasonably applied to flammable gas problems. The compounding of conservatisms and the large uncertainties in the data typically created unacceptable consequences (e.g., Van Vleet 1996). Though the consequences were highly improbable, the traditional bounding analyses could not quantify the probability, so the actual risk was unknown.

To quantify the risk, the uncertainty in the data and model assumptions had to be propagated through the analysis. Monte Carlo simulation was accepted as the most useful method to accomplish this. In a Monte Carlo analysis, the important input data for a model calculation are described as probability distributions rather than point values. Many calculations are performed (5,000-10,000 or more), each using a selection or "realization" of values chosen at random from the input probability distributions. The resulting large number of results forms an output probability distribution that represents the full range of uncertainty of all the inputs. This allows the level of conservatism of the answer to be chosen with some precision (e.g., 95\% confidence, $99 \%$ confidence, etc.).

The first application of this technique evaluated all 177 existing waste tanks (Hopkins 1995, Hodgson et al. 1996). The evaluation calculated the potential for the tank headspace to exceed the lower flammability limit based on estimates of the retained gas volume determined from the barometric pressure effect on surface level (Whitney 1995) and from surface level growth, 
assuming that a fixed fraction of this gas would be released. Even though the analysis correctly propagated the major uncertainties through the calculation, many inputs and assumptions were still deliberately conservative or even bounding. This made the evaluation over conservative and resulted in a recommendation to add 37 more tanks to the flammable gas watch list. The ensuing review (Johnson 1996) recommended the elimination of over-conservatism and development of a more mechanistic approach with less dependence on surface level measurements for evaluating the flammable gas hazard. No tanks were added to the watch list.

A much more ambitious, strictly probabilistic approach to estimating the overall risk of a release of radioactivity from the tanks was initiated in 1997. The "refined safety analysis" considered the whole series of postulated events from gas release through ignition, containment failure, ejection of radioactive material, and dispersion to the environment (Slezak et al. 1998). While the risk predicted with this model was very low, its complexity and the lack of knowledge or data on the processes of tank rupture and material ejection cast doubt on the conclusions. The extensive use of probability distributions derived by expert elicitation (i.e., opinion and "gut feel" based on accumulated experience) to quantify the breadth of uncertainty about some of the processes involved was suspect. Though the refined safety analysis methodology was eventually used in specific investigations, it was never incorporated into the safety authorization basis.

Though the concept of Monte Carlo simulation for flammable gas safety analysis had been accepted as a necessary replacement to traditional bounding calculations, the two attempts at applying it were not successful. The solution to this impasse demanded a clear, straightforward methodology and soundly based input distributions based as much as possible on tank data. The tank database also had to be carefully refined to include only what was reasonably accurate and applicable.

Appropriate sources of data for carefully defined waste types were established and documented in a watershed DOE workshop of flammable gas experts in 1999 (Barker et al. 1999). This compilation remains the best summary of available flammable gas source data to date. The general gas retention and release behavior of a tank was related to the waste types distinguished by physical configuration and the broadest possible characterization of the waste composition. Seven possible waste forms were identified: liquid-only, which cannot retain gas, and six more depending on whether the waste consisted of sludge, saltcake, or a mixture of the two and whether a deep supernatant liquid layer was present over the sediment. The workshop participants determined that, for the purposes of evaluating flammable gas retention and release, sludge and mixed sludge/saltcake waste generally behave like saltcake. This left only three functionally different waste types, liquid, liquid-over-solid ( $>1 \mathrm{~m}$ of liquid over sediment), and solid ( $<1 \mathrm{~m}$ of liquid over sediment). Probability distributions of retained gas volume fraction and gas release fraction were developed from tank data for the liquid-over-solid and solid waste types. These classifications and distributions form the basis for the current flammable gas safety evaluation method. 


\subsubsection{Current Waste Group Criteria}

The tank farm safety authorization basis is designed to apply administrative controls based on the potential flammable gas hazard. More stringent controls are applied to tanks with greater potential for the tank headspace to become flammable due to a gas release. The fundamental assumption, supported by a decade of experience and observation, is that actual or predicted waste conditions determine current or future tank behavior. The energy ratio (Eq. 1.8) and the buoyancy ratio (Eq. 1.18), along with other waste-based criteria, are used to classify tanks into waste groups according their flammable gas release potential, as outlined below (Barker and Hedengren 2004).

In the first step, the flammable gas waste group criteria first separate tanks into two large groups: Waste Group $\mathrm{C}$ for tanks that do not retain enough gas to make the headspace flammable even if all of it were released instantaneously, and Waste Groups A and B for tanks that do. Waste Group A tanks are then separated from Waste Group B because the A tanks exhibit spontaneous BDGREs. The concept is that Waste Group C tanks pose no hazard of either induced or spontaneous releases, Waste Group B tanks have the potential for a hazardous gas release induced by a large disturbance, and Waste Group A tanks may be subject to not only hazardous induced releases but spontaneous or induced BDGREs as well (Wells et al. 2002, Stewart et al. 2003).

The waste group classification calculation for a tank is performed in two steps. First, the flammability potential is calculated from the tank headspace volume, total sediment volume, retained gas volume fraction, and concentration of fuel gases (hydrogen, methane and ammonia) in the retained gas. Tanks without sufficient retained gas to be flammable are assigned to Waste Group C. Those remaining are checked for BDGRE potential, first with the energy ratio (Eq. 1.8) to determine whether a BDGRE could release gas, and second with the buoyancy ratio (Eq. 1.18) to see whether BDGREs will occur at all. Tanks with BDGRE gas release potential are assigned to Waste Group A and the rest to Waste Group B. A tank with insufficient retained gas to make the headspace flammable remains in Waste Group C even if it exhibits BDGREs.

The calculations separating the tanks into the three groups are performed as a Monte Carlo simulation wherein probability distributions describe the waste layer dimensions, liquid and sediment density, gas volume fraction, retained gas composition, and gas generation rate. A large number (typically 5,000) of classification calculations are performed, each with a different set of realizations of the input probability distributions. The resulting waste group classification is made at the $95^{\text {th }}$ percentile. If the tank classifies as Waste Group C in $95 \%$ or more of the trials, the tank is classified as Waste Group C. Tanks that fall into Waste Group A in more than $5 \%$ of the trials are placed in that group. All other tanks are classified as Waste Group B. 


\subsubsection{Problems in Applying the Waste Group Criteria}

The methodology described above is quite conservative in that it includes all important sources of uncertainty in the data, some of which can be quite large, and classifies tanks at the $95 \%$ confidence level. While this conservatism ensures safe waste storage, the single-shell tank retrieval mission requires concentration of liquid waste to the maximum permissible specific gravity stored at the maximum permissible waste level and sediment layer depth in a doubleshell tank. A higher specific gravity and deeper sediment increases the buoyancy ratio and brings the double-shell tanks closer to the Waste Group A criteria. At the same time, creation of new Waste Group A tanks is prohibited. This creates a conflict between keeping tanks out of Waste Group A and performing the operations necessary to implement the retrieval mission.

To effectively balance safety and operational necessity, it is important that the process for evaluating and assigning the tanks to waste groups be defensible and usable. But there are shortcomings in both the process and the data it uses. CH2M HILL performed a specialty assessment in 2004 to assess the process used to assign tanks to waste groups based on BDGRE hazard and to determine whether the process is defensible, whether input data are used appropriately, and how the process can be improved to enhance its usability in supporting the single-shell tank retrieval mission (Kirch and Meacham 2004). The observations of the specialty assessment that relate specifically to the BDGRE criterion in preventing creation of Waste Group A tanks are summarized below.

The problem that may have caused the most difficulty is the lack of margin in the application of the buoyancy ratio criterion. A tank operation such as a waste transfer or caustic addition is planned so that the buoyancy ratio, based on predicted post-operation tank conditions, does not exceed, but may approach, unity. No margin for error is included. When tank conditions are actually measured, some time after the operation, small differences from predicted values of supernatant liquid density or sediment depth, for example, can make the buoyancy ratio exceed unity. This indicates that a new Waste Group A tank has been created, violating the current operating agreement. Adjusting the leading coefficient of the buoyancy ratio equation so that the lowest value for a BDGRE tank is exactly one can cause a similar problem when conditions in the pivotal tank change slightly.(a) On the other hand, if a reasonable margin, say a factor of 2 , were applied, the operational buoyancy ratio criterion (now 0.5 instead of 1.0) would be prohibitively restrictive. This constriction makes the buoyancy ratio criterion seem overly sensitive and unusable.

A related problem is the lack of recent tank data. The ball rheometer, void fraction instrument, and retained gas sampler were last deployed in 1996-1997 (and in SY-101 in 1998). Data from continuous tank headspace gas monitoring ceased by 2002. There have been no new

(a) In Section 4 we recommend that the leading coefficient NOT be adjusted with changing conditions but be held constant to represent those conditions for which the BDGRE behavior was documented. 
waste core samples since about that same time. Only waste temperatures and surface levels continue to be recorded. This means that predictions of the effects of an operation often must be based on waste configuration and properties data almost a decade old. This almost ensures that new measurements will differ from predictions.

A structural problem in the Monte Carlo calculation amplifies the conservatism in the buoyancy ratio at the $95 \%$ confidence level. The denominator of the buoyancy ratio expressed by Eq. (1.18) contains the difference between the convective layer and sediment densities. When each of these densities is assigned values from independent probability distributions, the difference may become very small (i.e., high liquid density and low sediment density) and the buoyancy ratio very large. Because the sediment layer actually is 75 to 85 percent liquid by volume, it is not physically reasonable to use independent distributions. The sediment density ought to be a function of the liquid density so that the liquid density probability distribution determines the uncertainty of the sediment density. This will also reduce the perceived sensitivity of the buoyancy ratio to small changes in density.

The most far-reaching recommendation of the specialty assessment was to incorporate in the waste group criteria the fact that the current Waste Group A tanks are safe. This has already been demonstrated by the extensive study and documentation that resolved the Flammable Gas Safety Issue (Johnson et al. 2001). Therefore, the BDGRE criteria should be designed to avoid flammable headspace conditions, not simply to avoid a phenomenon (e.g., creation of another tank that behaves like AN-103). The objective of this report is to document revised BDGRE criteria that accomplish this recommendation.

\subsection{Outline of this Report}

This part of the report has summarized the history of flammable gas retention and release understanding and the development and application of safety analysis methodology. This report recommends a revision to the waste group classification criteria that cures some of the shortcomings of the current analysis system. Section 2 describes the relation of tank conditions to tank gas retention and release behavior, the sources of data available, and how they should be used. Section 3 describes the modeling basis and the input data and uncertainty distributions selected. The development of the final model and the BDGRE flammability criteria are discussed in Section 4, model validation and its range of applicability are discussed in Section 5, and cited references are listed in Section 6. Appendixes contain supplementary details. 


\subsection{Data Sources for Waste Conditions and Behavior}

Modeling waste tank gas release behavior is based on the fundamental assumption that the behavior observed in each tank, i.e., headspace hydrogen concentrations during gas releases, is related in some mechanistic way to measured waste conditions in each tank, e.g., layer depth and density. This assumption appears to be generally true but is complicated by the large range of gas release sizes that describe the behavior. Uncertainties are also large in the data describing the tank conditions. Nevertheless, both the tank behavior and the waste conditions must be described with as much precision as possible with the data available.

As described in Section 1, the available tank waste data are sufficient to develop simple physically based models to understand and predict BDGRE behavior. However, data that would allow for a more complete understanding of the BDGRE phenomena are lacking. For example, as discussed above, the bubble migration mechanism for the background gas release is not known and has not been observed in the laboratory. Specific waste parameters that would assist in understanding this phenomenon include sediment layer physical properties such as particle/agglomeration size and rheology, which may be extremely susceptible to sample handling effects (mixing/agitation, temperature changes, etc.). Waste rheology also plays a role in determining whether gas release will actually occur from a buoyant displacement as described by Eq. (1.8). This section describes the available data sources, application, and applicability.

\subsection{Thermocouples and Validation Probe}

Temperature histories in the tank waste provide heating and cooling information that is pertinent to waste chemistry changes, gas generation rates, and waste behavior. Temperature profiles in the tanks provide layer depth information. In addition, changes or lack of change in the temperature profile sometimes indicate where GREs originate.

The temperature of the waste is affected by the seasonal variations (typically on the order of a few degrees Fahrenheit in the waste, greater at the top and bottom of the waste). Waste temperature is not expected to be radially uniform at a given level in the waste except in the convective supernatant liquid, but the significant gradients are in all cases axial rather than radial. There is some heat transfer radially at the tank wall to the air in the annulus, but most of the heat transfer is in the axial direction, at the waste surface and at the tank bottom. At the waste surface, evaporative cooling and forced convection heat transfer due to the ventilation air flow are the primary means of heat removal. Beneath the tank, forced convection in the ventilation channels within the concrete pad on which the tank sits provides an efficient path for heat removal through the bottom of the tank.

The top-and-bottom cooling results in "camel hump" axial temperature profiles in the

sediment. Generally, the coldest temperature is indicated by the thermocouple near the tank 
bottom, and the hottest temperature is found near the middle of the sediment layer. The liquid supernatant layer is generally cooler than the sediment.

Temperature profiles are available from the multifunction instrument trees (MITs) (Drawing \# H-2-85122, Rev. 6) and the original thermocouple trees (Tran 1993). Tank SY-101 has two MITs. MITs have 22 thermocouples spaced 30-60 cm (12-24 in.) apart starting $10 \mathrm{~cm}$ (4 in.) from the tank bottom. These are usually read manually except in SY-101, where the readings are made automatically every 12 seconds. A validation probe that measures temperature every 10 $\mathrm{cm}$ (4 in.) with a high-precision resistance temperature detector (RTD) is operated occasionally to confirm thermocouple readings. The higher resolution of the validation probe profile gives an accurate sediment and crust layer thickness.

Readings from the old thermocouple trees are made manually. The thermocouples are spaced approximately every $125 \mathrm{~cm}$ (50 in.) vertically with the first reading near the tank bottom. But, because only one-half to one-third of the 20 thermocouples may be recorded, a large uncertainty exists in waste layer dimensions derived from these temperature profiles.

The temperature of the tank bottom is also measured by 24 thermocouples welded to the outer surface of the tank bottom plate and embedded in the concrete pad that supports the inner shell. While these thermocouples are not exposed to the waste and have a very slow response time, they can provide some information about the thermal state of the tank. They reveal a "donut" radial temperature profile with the highest temperature a little over halfway to the wall. This profile is a result of the annulus cooling flow entering at the bottom center of the tank. The bottom thermocouples are read manually approximately every week. Several of the thermocouples in each tank are inoperative.

The uncertainty in the temperature read by a thermocouple, whether on an MIT or thermocouple tree, is estimated at $\pm 1.8^{\circ} \mathrm{C}\left(3^{\circ} \mathrm{F}\right)$ (Brewster et al. 1995). However, experience with SY-101 data (Stewart et al. 1994, 1995) has shown that MITs are easily able to register temperature changes of $0.06^{\circ} \mathrm{C}\left(0.1^{\circ} \mathrm{F}\right)$. The uncertainty in the absolute temperature reading of the MIT validation probe is $\pm 0.2^{\circ} \mathrm{C}\left(0.3^{\circ} \mathrm{F}\right)$.

Temperature data is available for all of the double-shell tanks. However, the more definitive validation probe data are most typically available for the BDGRE tanks.

\subsection{Core Sample Analysis}

A major part of tank waste characterization is core sampling, which is used to obtain waste samples. Core sampling has been conducted in numerous Hanford tanks. The level of analysis of the samples varies from tank to tank and waste core to waste core. There are numerous core samples from the BDGRE tanks, some of which have been analyzed extensively. 
During core sampling, a stainless steel sampler is lowered down into the tank through a drill string, locked into a core barrel, and the drill string is then advanced (typically 19 inches). A spring-loaded piston is used to create negative pressure, which helps draw the sample into the waste column. Upon removal from the tank, the sample is placed in a cask and made available for extrusion and analysis.

Normally, the waste core samples are extruded horizontally in a hot cell, and varying degrees of analysis are conducted. These analyses provide crucial chemical and physical waste parameters that are pertinent to the current analysis, including liquid and sediment densities. Waste chemistry data are used to evaluate the undissolved solid content of the sediment and to compute gas generation rates. Core sample profiles also assist in determining waste layer configuration, although segment length adversely affects the accuracy of this method compared with the validation probe temperature profiles or the ball rheometer (Section 2.4). In addition to issues such as a sample from a single radial tank location representing a waste strata or analysis uncertainties, sample handling may have a significant effect on the results. For example, as presented in Section 3.2.2, temperature changes can affect liquid samples. Parameters fundamental to the understanding of waste behavior, such as particle size and rheology, have been shown to be affected as well.

As discussed in Section 1, the unknown slow bubble migration mechanism is described by assuming that the effective bulk viscosity in the sediment is proportionally indicated by the variation of shear strength, and that the energy required to yield a gob and release a significant fraction of its gas is proportional to the sediment's average shear strength. Shear strength data from the ball rheometer (Section 2.4) are used to represent the waste instead of those from waste core samples for essentially two reasons. First, and not as significant given the empirical correlation of the buoyancy ratio results to tank data, data pertaining to the variation of shear strength in the sediment with depth are simply not available from ex-tank core sample analyses. The available data are only for the BDGRE tanks. Second, direct-method ex-tank shear strength data for the BDGRE tanks are significantly different than those determined from in situ measurements and core sample extrusion analyses. (a)

\subsection{Retained Gas Sampler}

The retained gas sampler (RGS) system, which measured the in situ composition of gases retained in the waste, was a version of the universal core sampler (Section 2.2) that was designed specifically for sampling waste that contains gas. The complete details of the sample extraction process and results and uncertainties for tanks sampled with the RGS are presented in Mahoney et al. (1999). RGS data are arguably the definitive source for retained gas composition data. The only double-shell tanks sampled with the RGS were AN-103, AN-104, AN-105, AW-101, and

(a) Wells BE and SA Barker. 2003. Summary of Yield Stress in Shear Data for Hanford Waste. Letter Report TWS03.044, Pacific Northwest National Laboratory, Richland, Washington. 
SY-101. The sampling in SY-101 was conducted after the tank had been agitated with a mixer pump for approximately five years. (a)

\subsection{Ball Rheometer}

The ball rheometer concept originated from the need to measure in situ rheological properties in Hanford DSTs. It has been deployed only in the BDGRE DSTs. As with the RGS, deployment in SY-101 occurred after operation of the mixer pump. Ex-tank waste sample handling can alter its rheological characteristics, as can the temperature changes that occur after the sample is removed from tank. In contrast, the rheology of the waste material can be estimated in situ directly from the drag force on a ball as it moves through the waste at various speeds. Liquid density measurements and layer interface identification may also be made with ball rheometer data.

The ball rheometer system consists of a 71-N (16-lb), 9.12-cm (3.6-inch) diameter tungsten alloy ball tethered to a steel cable that is wound onto a spool. The cable runs through a guide that distributes the line across the width of the spool, so only a single layer of cable is needed on the spool. Cable deployment and retrieval are performed with electric motors, clutches, reduction gears, and a driveline. The pulley rests on a load cell that measures the tension in the cable plus the pulley weight and any friction in the pulley when it is in motion. The ball may be moved at varied speeds through the waste, or static measurements can be made.

Cable tension data are measured with a nominal 445-N (100-1b) full-scale load cell. Because the cable runs over a pulley, the effective load limit is actually $222 \mathrm{~N}(50 \mathrm{lb})$, half the load cell value. The published error for this load cell is $0.1 \%$ of the full-scale reading, or $0.2 \mathrm{~N}$, regardless of load. Indeed, the data show that the standard deviation of measurements made under static and dynamic conditions is, in almost all cases, $0.2 \mathrm{~N}$.

The liquid density measurement depends on both ball volume and buoyancy force. To determine density, a reference measurement of the ball in air is required. This value is subtracted in all density determinations. The reference measurement is also uncertain by $0.2 \mathrm{~N}$, but because it is used in all density determinations it represents a systematic error. The uncertainty in the ball volume is $1 \%$, also a systematic error.

Ball position is determined with a multi-turn absolute rotary optical encoder that rotates with the cable spool. The encoder has 4,096 steps per revolution; thus, the positional accuracy is within $0.01 \mathrm{~cm}$, a negligible error, if the cable spool diameter is known exactly. Uncertainty in the cable spool diameter is the largest source of error in position. The spool diameter, including the cable, was measured at $16.71 \mathrm{~cm}$ and is believed to be accurate to within $0.03 \mathrm{~cm}$. With

(a) BDGRE behavior in SY-101 was halted due to operation of the mixer pump. 
$1,000 \mathrm{~cm}$ of deployed cable, a positional error of about $2 \mathrm{~cm}$ is possible due to this uncertainty. Errors in the ball velocity are less than $1 \%$ and are negligible in their effect on determining rheological parameters, where force measurement errors and corrections to the load cell data dominate the uncertainties.

Additional uncertainty exists in the elevation of home position above the tank bottom. The elevations of the top of the riser and tank bottom are taken from design drawings that represent conditions before the tanks were filled. Attempts have been made to reference the ball position to the indicated waste level, but, because the ball buoyancy transition indicates the free liquid level and the waste level device senses the top of the crust layer, a difference can be expected. For the six tanks tested, the liquid level indicated by the ball averages $2 \mathrm{~cm}$ higher than the surface level, which is opposite what would be expected for a floating crust. In SY-101, the ball indicated the liquid surface to be $2.4 \mathrm{~cm}$ below the level measured by the Enraf ${ }^{\mathrm{TM}}$ gauge. Considering all sources, we ascribe an uncertainty of $\pm 4.5 \mathrm{~cm}(1.8 \mathrm{in}$.), or one ball radius, to all waste elevations indicated by the ball. Finally, waste disturbance as a result of the ball rheometer deployment may also affect results. For example, density gradients in the liquid are attributed to crust layer disturbance during the sampling event causing solids to settle (Hedengren et al. 2000).

With consideration of the discussed uncertainties, ball rheometer liquid density and rheological data are expected to be the most representative of in situ conditions given that the data is collected in-tank. Waste layer boundaries identified with the ball rheometer are also more definitive.

\subsection{Neutron and Gamma Probe}

A small-bore neutron and gamma probe was developed to operate inside the tube designed for the MIT validation probe. Neutron and gamma probes have been deployed in the majority of the single shell tanks and the historic BDGRE double-shell tanks. The neutron count measured by the probe indicates the concentration of hydrogen atoms surrounding the probe. Thus, the neutron $\log$ is essentially a measure of the relative fraction of water, which is a surrogate for liquid waste. A low neutron count may indicate the presence of gas or a high solids volume fraction. A high neutron count would indicate a dilute region. The neutron probe is quite sensitive at high gas fractions (20 to $50 \mathrm{vol} \%$ ) but does not discriminate low gas fractions $(<10$ vol\%) very well.

The gamma count indicates the relative amount of cesium-137, which is the main gamma source in the waste. Because cesium is quite soluble, the gamma count is to some extent a surrogate for liquid with a low gamma count indicating either gas or a high solids fraction. However, unlike the neutron log, a dilute solution containing less dissolved cesium also produces a low gamma count. The gamma response is more linear than that of the neutron probe, but a sharp definition of layer boundaries still requires a large difference in liquid content. 
In SY-101 prior to mitigation, the high void fraction at the base of the crust layer and moderate void fraction elsewhere in the crust, and the neutron and gamma logs provided very good information about the detailed structure of the crust. The neutron/gamma probe has not been so useful in the other tanks. Except for AN-103 and AW-101, the crust layers are generally about $0.5 \mathrm{~m}$ thick and have a relatively low void fraction compared with 101 . The nonconvective layers of all the tanks have a much lower void fraction that is difficult to detect with these devices. Nevertheless, in combination with temperature profiles and other data, the neutron/ gamma data provide confirmatory evidence of the location and characteristics of the crust and nonconvective layers of these tanks.

\subsection{Surface Level}

The elevation of the waste surface above the tank bottom is a key indicator of tank status and waste behavior and is measured in all tanks. Tanks with a high waste level are generally more hazardous because they store gas at a higher pressure, and less headspace is available to dilute episodic gas releases. A gradual increase in waste level may indicate accumulation of stored gas, while a quick drop may be a sign of a sudden gas release. A gradual drop is usually associated with evaporation of liquid. The total stored gas volume in a tank can be computed from the correlation of waste level changes with barometric pressure fluctuations.

Waste surface levels have historically been measured with three different devices: the Food Instrument Corporation (FIC) contact probe, a manual tape, and the Enraf ${ }^{\text {TM }}$ buoyancy gauge. The FIC and manual tape level readings are available in each double-shell tank essentially from the time they started operation in the late 1970s. But only the period since the mid-1980s, after the last fill, is of interest as evidence of current and future gas release behavior. Enraf level gauges have been installed only since about 1995, so their history is limited. Level measurements may be either manual or automatic. Early data were weekly; daily or once-per-shift readings have been available since the late 1980s.

The various instruments report different values and trends, mainly because of the irregularity of the crust surface but also because of the peculiarities of the measurement methods. The FIC and manual tape must be flushed occasionally to remove accumulated waste deposits. The Enraf buoyancy gauge is not subject to buildup of waste deposits, nor does it disturb the surface on which it rests. This makes the Enraf potentially the most reliable level indicator.

The absolute accuracy of any of the waste level instruments depends on the degree of surface irregularity and the uncertainty in the reference position. Combined, these could produce a systematic error of 2 to $20 \mathrm{~cm}$ ( 1 in. to 8 in.) depending on the tank. Sensing level changes is far more important than absolute accuracy, however. A study of SY-101 data (Brewster et al. 1995) concluded that the Enraf correlated most closely with gas releases and was the most precise level instrument. It records level in $0.025-\mathrm{cm}(0.01-\mathrm{in}$.) increments and is easily able to resolve level changes to within $\pm 0.25 \mathrm{~cm}(0.1 \mathrm{in})$. The FIC usually correlates closely with the Enraf and 
reasonably well with gas release in SY-101. It records level in 0.25-cm (0.1-in.) increments, and its sensitivity is estimated at $\pm 0.6 \mathrm{~cm}(0.25 \mathrm{in}$.) for nearly consecutive readings, but waste deposit growth and periodic flushing adds an uncertainty of $\pm 5 \mathrm{~cm}(2$ in.) in long-term values.

The manual tape is typically read in $0.64-\mathrm{cm}(0.25$-in.) increments; therefore, it senses changes only to within $\pm 2 \mathrm{~cm}$ ( 0.75 in.) at best. Because of this low sensitivity, the manual tape was not correlated with any of the other instruments or with gas release in the SY-101 study.

\subsection{Headspace Gas Monitoring}

The concentrations of hydrogen and other flammable gases in the tank headspace historically have been monitored to ensure that the headspace is safe and to increase our understanding of the mechanisms for gas release and dilution. Headspace gas monitoring systems have been installed on most of the tanks that were on the flammable gas watch list.

Headspace gas monitoring is the primary means of identifying and quantifying gas release events. In conjunction with waste level histories, headspace ventilation rates, and retained gas concentrations, gas release events may be quantified by their peak hydrogen concentration, total release volume, gas release rate, and time to peak gas release rate. Headspace gas monitoring and associated data may also be used to quantify gas generation. The ability to determine these gas generation and release parameters and their accuracy depends on the level of instrumentation in the tank.

Several different gas monitoring systems have been used, including various configurations of the standard hydrogen monitoring system (SHMS) and gas monitoring system (GMS). The more advanced SHMS may be outfitted with two Whittaker ${ }^{\mathrm{TM}}$ cells, ${ }^{\text {(a) }}$ two gas chromatograph thermal conductivity detectors (GC-TCDs) and an IR monitor. The Whittaker cells and GC-TCDs monitor hydrogen, nitrous oxide, and methane, and the IR monitor detects ammonia. The GCS, an upgraded GMS, has an IR monitor and two GC-TCDs. Hedengren et al. (2000) report the measurement accuracies and ranges.

The historic BDGRE tanks have had various gas monitoring systems installed, (b) some of which are not sufficiently accurate to completely quantify the gas release events. In addition, headspace ventilation rates were not continuously monitored except in SY-101. Thus, the most quantifiable gas release event parameter is the measured peak hydrogen concentration. Gas generation rates are typically not accurately quantifiable from field data, if available at all.

(a) Whittaker is a trademark of Whittaker Corporation, Garden Grove, California.

(b) SY-101 and SY-103 have had gas monitoring since 1992, while AN-103, AN-104, AN-105, and AW-101 have had gas monitoring since 1994. 


\subsection{Other Data Sources}

Other pertinent data sources may be available on a tank-by-tank basis. As mentioned above, headspace ventilation rates are beneficial to gas generation and release analyses but are typically not measured. Evaporation effects on a tank may be quantified with headspace humidity, typically not measured, and ventilation rates. Headspace pressure, if available, may be related to ventilation rates and gas release events.

Records for any in-tank activity, instrument installation or cleaning, waste transfers, waste surface video or photos, have historically been scrutinized for useful information regarding tank behavior. For example, the multi-gob phenomenon of BDGREs was first identified by in-tank video from SY-101. Waste rheological data have been gleaned from such obscure sources as estimated operator effort for in-tank instrument deployment (estimating the shear strength of the crust in SY-101, Rassat et al. 2000), and horizontal core sample extrusion videos (Gauglitz and Aikin 1997, Rassat et al. 2003). 


\subsection{Model Basis}

The task at hand is to derive a model to predict tank headspace hydrogen concentration resulting from spontaneous gas releases based on available data that describe the waste configuration and properties. Ideally, a model should be a close mathematical representation of well-understood processes in a well-defined physical system for which a large body of experimental or observational data is available to validate it. Using the scientific principle, the data suggest relationships or principles forming a model that is then tested against a different set of data. However, it is often difficult to apply the scientific principle rigorously when data are few and systems incompletely understood.

Only six tanks have recorded BDGREs, and the events vary widely in size and frequency. The corresponding waste data have a relatively large uncertainty. At the same time, our knowledge of the processes involved is incomplete. While a slow gas migration mechanism is required to explain why some tanks have BDGREs and most do not, the exact mechanism is not known. While we have a conceptual model of a BDGRE that is consistent with the tank data, the processes that determine how many gobs go buoyant and how large they are remain unclear. In fact, only the larger releases can be positively identified as BDGREs in the first place. The smaller ones may well be the result of some other mechanism. These hindrances affect the model basis, development, validation and its actual application.

Section 3.1 evaluates several options for the mathematical formulation and analysis method. The data uncertainty distributions are derived and documented in Section 3.2.

\subsection{Mathematical Formulation}

With a less-than-perfect understanding of the physics of BDGREs and a less-than-perfect database to support it, an important question is whether physics or statistics govern model development. Which is most defensible? Because the model will be applied over a wide range of conditions that may not represented by the current data set and model assumptions, it is also important that the basis be clear and simple. A purely physics-based model would be desirable because it would then be clear exactly what physical mechanisms are considered and what assumptions are made. On the other hand, a model is defensible only if it can fit the available data reasonably well. This section presents some options and a justification for the one selected.

\subsubsection{Tank Gas Release Data; Hot and Cold Periods}

Typical gas release volumes of AN-103, AN-104, AN-105, and AW-101 decreased markedly during 1996-1997 after an increase in annulus ventilation rate caused these tanks to begin cooling. The average sediment temperature in these tanks decreased by 5 to $7.5^{\circ} \mathrm{C}$ by 1999 , decreasing the gas generation rate by 30 to 50 percent and, in all tanks but AW-101, the 
buoyancy ratio by 12 to 20 percent. The buoyancy ratio in AW-101 is actually increased in the "cold" state due to an apparent increase in sediment depth, as presented in Section 3.2.4. Because the buoyancy ratio change was consistent with the change in gas release behavior (with the exception of AW-101), the tank and BDGRE data were separated into "hot" and "cold" periods, before and after the ventilation change, respectively.

However, even though the tanks may have reached a reasonably steady thermal state by 1999, their gas release behavior may still have been evolving. The gas generation rates in the "cold" tanks would increase the gas volume fraction only 1 to 1.5 vol\%/year if all of it were retained. With an average neutral buoyancy gas volume fraction of $10 \mathrm{vol} \%$ and a gas release fraction of 0.5 , the time between BDGREs for a typical gob of sediment would be 3 to 5 years. This indicates that, if the temperature decrease began to have a significant effect in 1997, the change gas release behavior would not be complete until 2000 to 2002 at the earliest. There is no gas monitoring data past 2002 to confirm or deny this.

\subsubsection{Buoyancy Ratio as an Indicator of BDGRE Size}

It has long been recognized that the buoyancy ratio of Eq. (1.18) is much larger for SY-101, whose gas releases recorded from late 1989 through July 1993 stand out in magnitude from all other tanks. The derivation of the buoyancy ratio did not consider the size of BDGREs, only whether they occur. Nevertheless, the buoyancy ratio is already incorporated in the safety authorization basis and has a demonstrated ability to separate BDGRE tanks from the rest.

The relationship between the buoyancy ratio (BR) and the release volume calculated directly from the measured peak hydrogen concentration is shown in Figure 3.1. The release volume is considered to eliminate the effects that hydrogen concentration and headspace volume have on the peak hydrogen concentration. The calculated release volume is plotted against BR - 1 so that non-BDGRE tanks have negative values. A quadratic curve fit (forced through zero) to the release volume for all recorded BDGREs is shown as the dashed line. The $\mathrm{R}^{2}$ value for the fit is 0.804. The mean gas release volumes are plotted against BR-1 for hot and cold tanks. The BR-1 values are also shown for the non-BDGRE tanks at a fixed zero release volume. AN-107 is the one non-BDGRE tank with BR-1 $>0$ as is addressed in Section 5.

The safety hazard of a BDGRE is not measured by the calculated release volume but by the elevation of the headspace hydrogen concentration resulting from the release, which is the fundamental quantity measured in-tank. In Eq. (1.2), the peak headspace hydrogen concentration is equal to prompt release volume multiplied by the fraction of hydrogen in the retained gas and divided by the headspace volume. Thus, if the buoyancy ratio is related to the prompt gas release volume as $V_{R}=f(B R-1)$, the peak hydrogen concentration should be related to BR-1 as

$$
\left[\mathrm{H}_{2}\right]_{\text {peak }}=\mathrm{f}\left\{(\mathrm{BR}-1)\left[\mathrm{H}_{2}\right]_{\text {gas }} / \mathrm{V}_{\mathrm{HS}}\right\}
$$




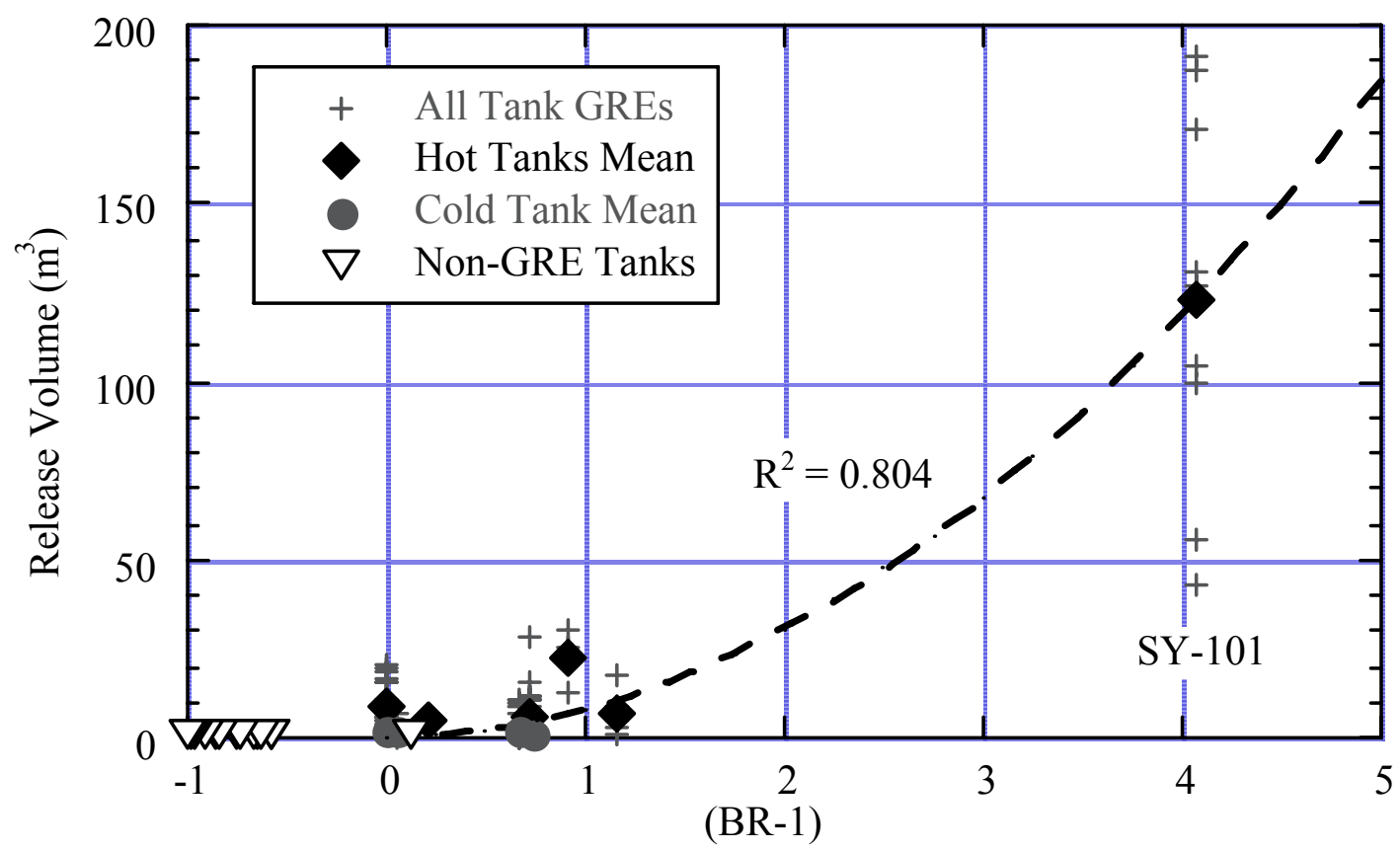

Figure 3.1. Calculated Gas Release Volume Versus BR-1

Eq. (3.1) is plotted versus the measured peak hydrogen concentration in Figure 3.2. The quadratic curve fit (forced through zero) to all the BDGREs, shown as the dashed line, has an $\mathrm{R}^{2}$ value of 0.786 . The position of the tanks has shifted with respect to Figure 3.1 because of differences in headspace and retained gas hydrogen fraction.

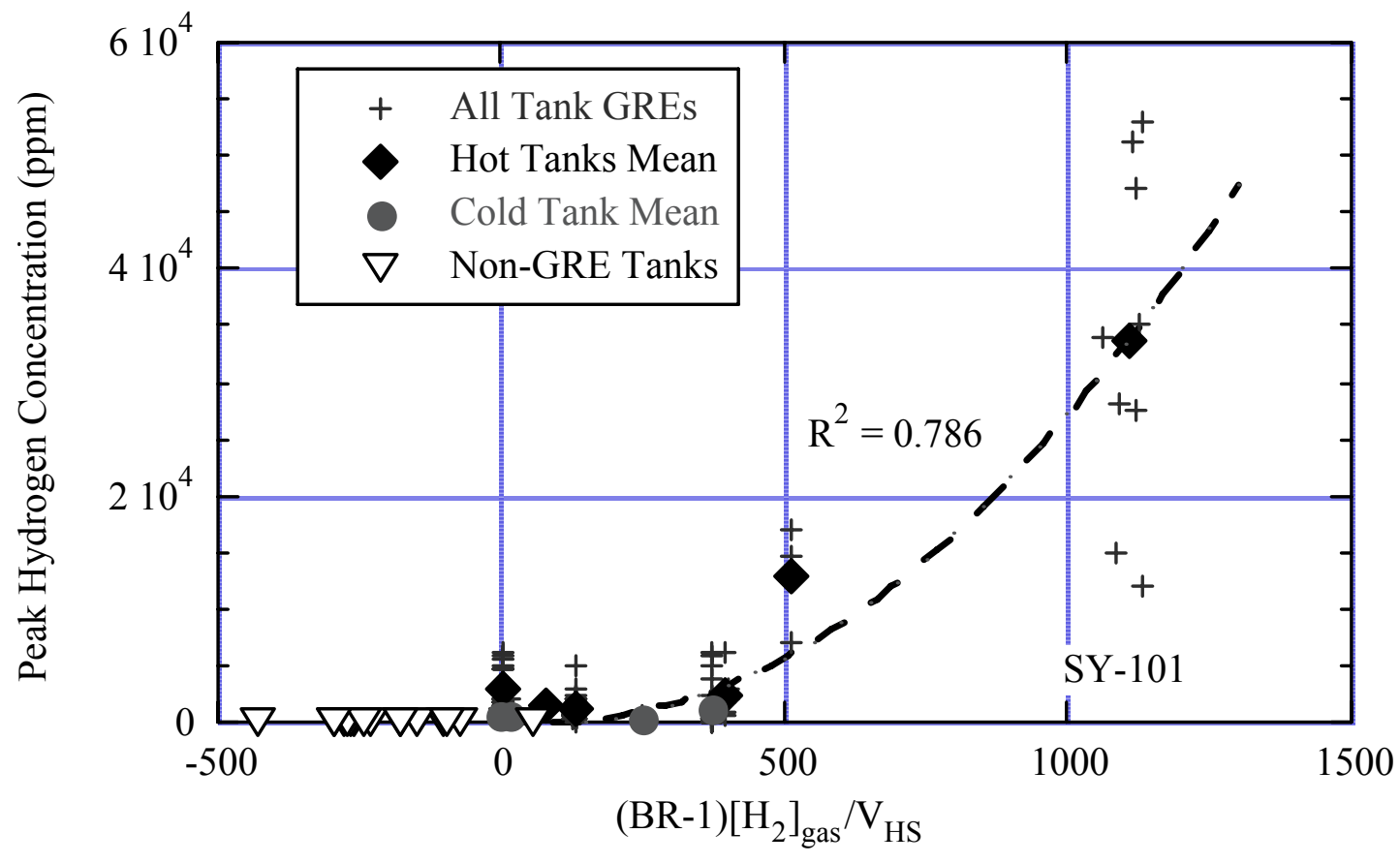

Figure 3.2. Measured Peak Hydrogen Concentration Versus (BR-1) $\left[\mathrm{H}_{2}\right]_{\text {gas }} / \mathrm{V}_{\mathrm{HS}}$ 


\subsubsection{Physical Model Based on Rayleigh-Taylor Instability}

As an alternative to the buoyancy ratio, a model has been developed to predict the size of a gob of sediment undergoing a BDGRE based on the theory of instability of uniform plane layers

of different densities (see Appendix A). The model gives the most probable volume of a fulldepth gob in a weak sediment layer(a) as

$$
V_{\text {gob }}=B \frac{\tau_{y} H_{S}}{\rho_{S}\left(1-\alpha_{N B}\right)}
$$

where B is a proportionality constant and $\tau_{\mathrm{y}}$ is the sediment yield stress. ${ }^{(b)}$ Substituting the gob volume from Eq. (3.2) into Eq. (1.3) for the total gas release volume and including the yield stress in the leading coefficient, $\mathrm{D}$, gives

$$
\mathrm{V}_{\mathrm{R}}=\mathrm{D} \frac{\mathrm{H}_{\mathrm{S}}}{\rho_{\mathrm{S}}} \frac{\alpha_{\mathrm{NB}}}{\left(1-\alpha_{\mathrm{NB}}\right)}\left(\frac{\mathrm{p}_{\mathrm{gas}}}{\mathrm{p}_{\mathrm{HS}}}-1\right)
$$

Though this model most closely represents the actual physics describing a full-depth gob that breaks away from a uniform sediment layer and the process of gob breakup and gas release at the waste surface, it does not fit the data very well. It is obvious from in-tank video that the very large BDGREs that occurred in SY-101 before July 1993 consisted of a number of gobs releasing in series. Eq. (3.3) fits the calculated gas release volume from SY-101 only if each release in this tank is assumed to consist of five gobs.(c) On the other end of the spectrum, the gas release volume predicted for AN-103 is about three times larger than the data show. This might indicate that AN-103's gas releases are not BDGREs or that they occur over only part of the depth of the sediment layer.

The comparison of Eq. (3.3) with the constant $\mathrm{C}$ adjusted to minimize the error for tanks AN104, AN-105, AW-101, and SY-103 is shown in Figure 3.3. Note the logarithmic scale on the release volume. This model would require an additional theory to predict the number of gobs in a release as well as their size.

(a) A gob requires a gas volume fraction slightly higher than the neutral buoyancy value to break away and experience a BDGRE. In the "weak limit" this increase in gas volume fraction is ignored (see Appendix A).

(b) The average yield stress in the sediment is approximately the same in all six BDGRE tanks and thus amounts to a constant that can be subsumed into the leading coefficient.

(c) While the gas monitoring data show that some releases in tanks other than SY-101 occasionally consist of two gobs, assuming a single gob per BDGRE is accurate except in SY-101. 


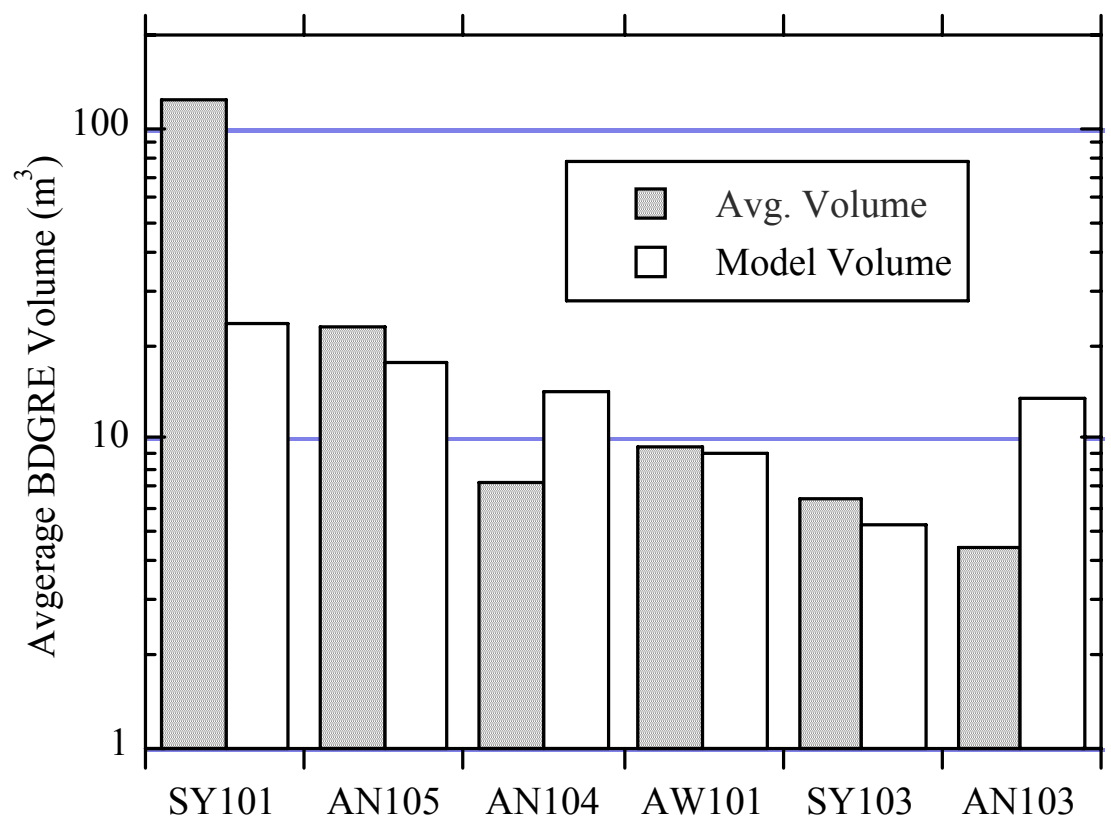

Figure 3.3. Performance of Rayleigh-Taylor Gas Release Model

\subsubsection{Modified Buoyancy Ratio Model}

Because the buoyancy ratio appears to account for the difference between the multiple-gob BDGREs of SY-101 and the mostly single-gob releases of the other tanks, it might be beneficial to use the product of the buoyancy ratio and the gob release model. Multiplying Eq. (1.18) and (3.3) and substituting Eq. (1.1) for $\alpha_{\mathrm{NB}}$ gives the following model for release volume:

$$
\mathrm{BRV}_{\mathrm{R}}=\mathrm{C} \frac{\mathrm{H}_{\mathrm{S}}^{3}}{\rho_{\mathrm{L}} \rho_{\mathrm{S}}}\left(\frac{\mathrm{p}_{\text {gas }}}{\mathrm{p}_{\mathrm{HS}}}-1\right)\left(\frac{\mathrm{GT}_{\mathrm{S}}}{\mathrm{p}_{\text {gas }}}\right)^{1 / 3}
$$

The coefficient $C$ is adjusted so that the minimum value of $\left(B R V_{R}-1\right)$ for the BDGRE tanks is zero. Figure 3.4 plots the measured peak hydrogen concentration versus $\left(B R V_{R}-1\right)$ $\left[\mathrm{H}_{2}\right]_{\text {gas }} / \mathrm{V}_{\mathrm{HS}}$. The quadratic curve fit to all BDGREs, shown as a dashed line, has an $\mathrm{R}^{2}$ of 0.780 .

This formulation is a slightly better fit than the buoyancy ratio and clearly separates all of the non-BDGRE tanks, including AN-107. However, Eq. (3.4) is likely to be overly sensitive to the sediment depth because it appears to the third power. The sensitivity would be exacerbated in practice because the sediment depth is very hard to predict and measurements are relatively uncertain. Also, the conceptual advantage of the mechanistic basis provided by the gob release model is lost when multiplied by the buoyancy ratio, which is not related to the mechanics of gob release. We conclude that there is little advantage (basis and fit) and potentially a serious disadvantage (sensitivity to $\mathrm{H}_{\mathrm{S}}$ ) to application of Eq. (3.4). 


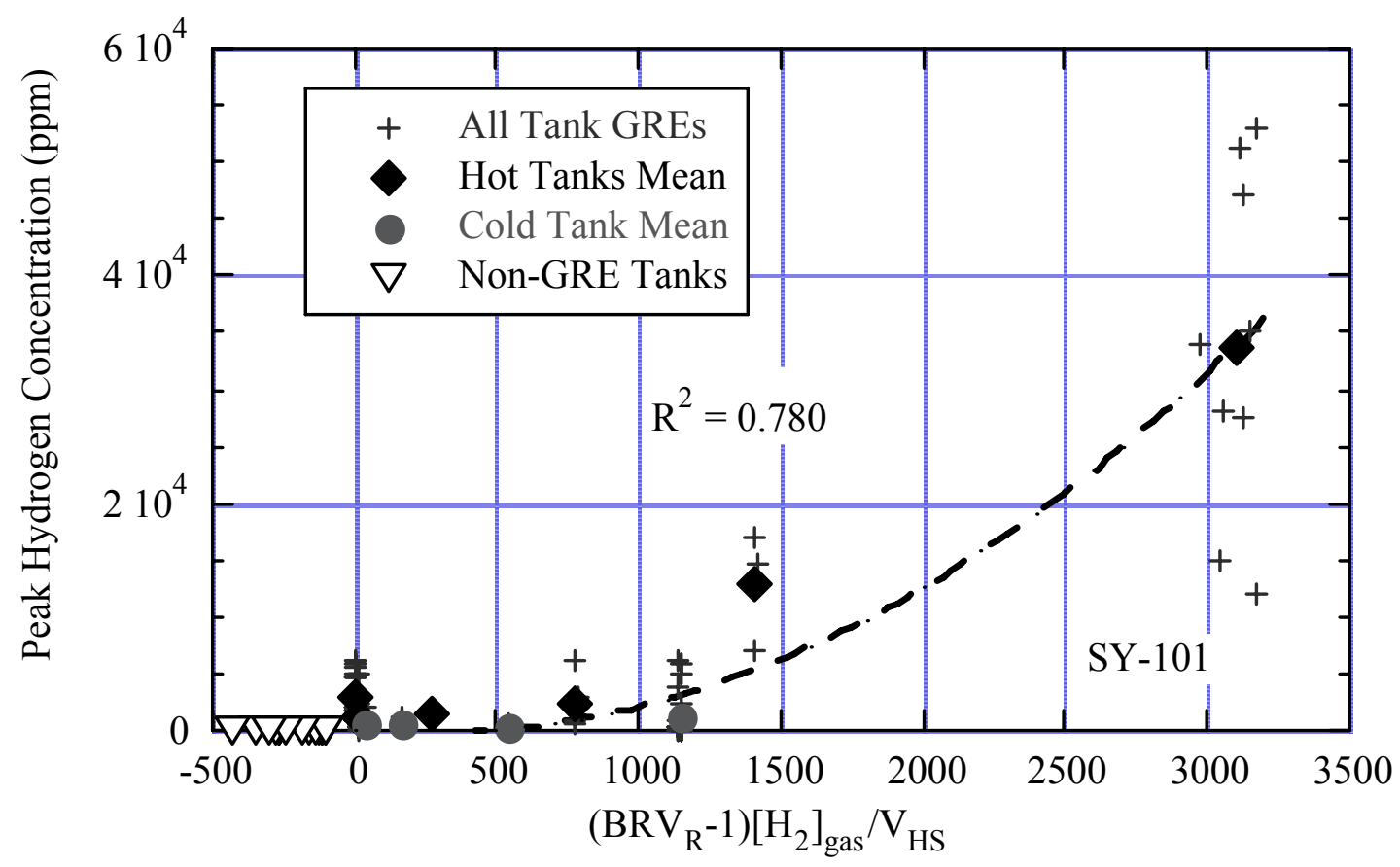

Figure 3.4. Measured Peak Hydrogen Concentration Versus $\left(\mathrm{BRV}_{\mathrm{R}}-1\right)\left[\mathrm{H}_{2}\right]_{\mathrm{gas}} / \mathrm{V}_{\mathrm{HS}}$

\subsubsection{Strictly Empirical Model}

Another option is to simply fit a grouping of the available input variables, such as density, temperature, gas generation rate, etc., to the data. For example, a least squares fit to the gas release volumes for the six hot tanks using the variables found in Eq. (3.4) produces the following linear empirical model:

$$
\left(\mathrm{V}_{\mathrm{R}}\right)_{\mathrm{E}}=-1351+1654 \mathrm{G}+1.7 \mathrm{~T}_{\mathrm{S}}-0.75 \rho_{\mathrm{S}}+1.3 \rho_{\mathrm{L}}+33 \mathrm{H}_{\mathrm{S}}+70\left(\frac{\mathrm{p}_{\text {gas }}}{\mathrm{p}_{\mathrm{HS}}}-1\right)
$$

A plot of the measured peak hydrogen concentration versus $\left(\mathrm{V}_{\mathrm{R}}\right)_{\mathrm{E}}\left[\mathrm{H}_{2}\right]_{\text {gas }} / \mathrm{V}_{\mathrm{HS}}$ for the BDGRE tanks is shown in Figure 3.5. The $\mathrm{R}^{2}$ value for a linear fit to all BDGREs is 0.805 . Eq. (3.5) fits the data somewhat better than the buoyancy ratio, and all of the non-BDGRE tanks are separated by a wide margin (too far to include on the plot). However, the cold tank AN-104 is nonconservatively predicted to have a negative peak hydrogen concentration. Also, though neither the buoyancy ratio nor the empirical model is based on the physics of gas release, the former is at least derived from gas retention theory and is consistent with several in situ measurements. Again, we conclude that the buoyancy ratio is the most appropriate basis model, all things considered. 


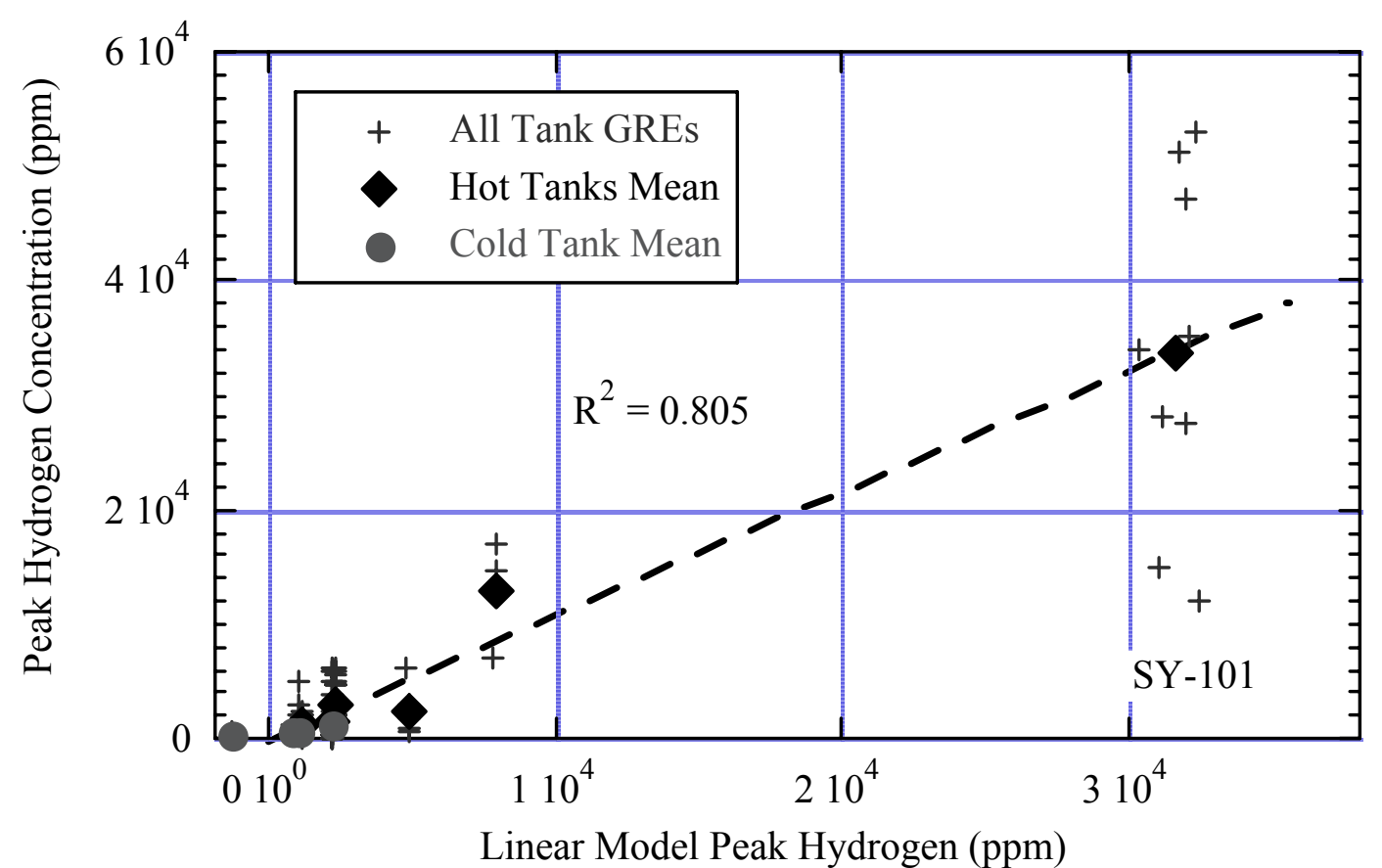

Figure 3.5. Measured Peak Hydrogen Concentration Versus $\left(\mathrm{V}_{\mathrm{R}}\right)_{\mathrm{E}}\left[\mathrm{H}_{2}\right]_{\mathrm{gas}} / \mathrm{V}_{\mathrm{HS}}$

\subsubsection{Hot and Cold Tank Gas Release Data}

The cold tanks are included with the hot tanks in developing the gas release model even though the cold tank data may not represent steady-state behavior. The average gas release volumes of the cold tanks decreased much more than their buoyancy ratios, and the smaller ones may not have been BDGREs at all. One cold tank, AW-101, actually has a higher buoyancy ratio than its hot counterpart. This might indicate that the change in behavior is the result of an unknown process not described by the current waste data and models. On the other hand, the decrease in heat load and gas generation rate by decay of radioactivity over the very long BDGRE cycle times makes all gas release behavior transient in some sense. The gas release behavior of SY-101 appears to have gone through several changes throughout its 13-year BDGRE history (Antoniak 1994). The distinctly different behavior of the cold tanks may even represent the norm.

Separate from the issue of validity, the trend of the gas release data is dominated by two hot tanks, SY-101, whose releases are about an order of magnitude larger than any of the other tanks, and AN-105, which is the only tank besides SY-101 with releases exceeding 10,000 ppm of hydrogen. Compared with these two tanks, the cluster of other hot and cold tanks forms a massive third data point representing very small releases (see Figure 3.2, for example). The result is that including the cold tanks has only a small effect on the resulting model. However, because the cold tanks do represent a distinct behavior described by at least small differences in tank conditions, they will be included in developing the model. 


\subsubsection{Model Development Method}

The model inputs required to calculate buoyancy ratio have significant, quantified uncertainties that must be included in the final result. We must account for all the parameter uncertainties and establish the overall probability distribution for the model predictions. But, rather than performing a deterministic calculation with all inputs set to bounding values, which has no physical or statistical meaning, uncertainties can be propagated through the calculation accurately using a Monte Carlo simulation. In this method, a large number of model simulations are run with input sets selected from their respective distributions. The collection of output values from all the model simulations then forms the overall probability distribution of the result.

A Monte Carlo simulation approach was used to derive the model relating the buoyancy ratio to the peak hydrogen concentration. For each tank modeled with the buoyancy ratio, 10,000 simulations were conducted. The result is a set of 10,000 model outputs that constitutes a probability distribution over those results. This allows us to predict the probability of a given result given the input probability distributions.

Each value used for the inputs is randomly sampled from an infinite population based on the specified distribution, as described in Section 3.2. The commercial code $\mathrm{S}+(\mathrm{a})$ was used to generate these inputs. A Visual Basic code written around the Excel ${ }^{\circledR}$-based model was used to generate results for each of the 10,000 simulations. These buoyancy ratio results from the Monte Carlo simulation are then used, as shown in the single-value examples of Section 3.1.1, to model the trends in gas release volume and peak hydrogen concentrations in the BDGRE tanks.

\subsection{Source Data Uncertainty Distributions}

Specific uncertainty distributions and values describing waste properties and configuration are determined from the data sources presented in Section 2.2. For some measurements such as surface level, data interpretation is limited because there is a single data source and relative ease of measurement, and thus determination of a value is relatively straightforward. For other items such as waste layer density, numerous and sometimes differing data sources are available. Sampling conditions may also play a significant role, further confounding data interpretation.

In the following subsections the data, bases, assumptions, and uncertainties in the form of distributions for each parameter for the historic BDGRE tanks AN-103, AN-104, AN-105, AW-101, and SY-103 are elucidated. Generally, when illustration is required, the analyses for AN-103 are presented in detail with supporting information from the remaining tanks in some instances. Given data uncertainty, no attempt is made to track significant figures. Preremediation SY-101 data are typically taken from Meyer et al. (1997), Rassat et al. (2000) and Hedengren et al. (2000) and presented with a summary of the complete data set in Section 3.2.8.

(a) S+ Version 6.1, Insightful Corporation, Seattle, Washington. 


\subsubsection{Average Sediment Temperature}

The average sediment temperature is used to convert the molar gas generation rate to a local volumetric rate in the buoyancy ratio and is applied through $\mathrm{Hu}$ 's model ( $\mathrm{Hu} 2005)$ to estimate the gas generation rate for the hot and cold tanks, except for SY-101. The average temperature is computed simply as the average of all daily readings of thermocouples in the sediment over the hot and cold periods of each tank. Only the original thermocouple trees were available through 1997, when data from the new multifunction instrument trees began being recorded. Accordingly, the hot tanks generally use data from the original thermocouple trees. The cold tanks also use these data to make the averages consistent for both hot and cold periods. The uncertainty in these averages is low, and the seasonal temperature variation is included in the average. Therefore, the average sediment temperature is taken as a constant, and no uncertainty distribution is applied. The average sediment temperatures are listed in Section 3.2.8.

\subsubsection{Layer Average Densities}

Supernatant and sediment layer density distributions are based on examination of all available data. These data include core and grab sample analyses and ball rheometer results. Uncertainties are developed based on data scatter rather than instrumentation uncertainty, which is typically insignificant by comparison. Indications of changes in layer density due to temperature effects are considered. Core and grab sample data are taken from the Tank Waste Information Network System (TWINS). (a) Ball rheometer data are presented in Hedengren et al. (2000).

\subsubsection{Data Sorting}

In AN-103, the example tank, Cores 166 and 167 were taken in September 1996 and Core 274 in February 2000. The ball rheometer was deployed in AN-103 in 1995. Cores 166 and 167 and the ball rheometer results are deemed applicable to hot tank conditions as described in Section 3.1.1, while Core 274 may be considered applicable to cold tank conditions.

The core sample analyses from TWINS contained 336 results potentially applicable to the current analysis. With three core sampling events of approximately 19 segments apiece, and several distinct analyses for possible multiple components of each segment, data analysis is challenging.

Therefore, for each result, the applicability to determining layer density was initially evaluated by considering the constituent name (e.g., specific gravity, solid density, solid weight) and sample description (e.g., AN-103 Core 166, Segment 11 Drainable Liquid; AN-103 Core 167, Segment 15 Upper Half Solids). Forty-four individual constituent names and 124

(a) TWINS, Tank Waste Information Network System, http://twins.pnl.gov/twins3/twins.htm. 
individual sample descriptions were identified. A 124 by 44 matrix of the data was generated such that, for example, the first instance of the constituent "specific gravity" could be seen for each applicable sample description, and so on.

This analysis retained core sample data not pertinent to waste layer density determination such as "AN-103 Field Blank" data and "Liner Liquid Volume(s)." Thus a secondary filtering step was used to remove entries of this sort and resulting "zero" rows or columns of the matrix (e.g., removal of the "Liner Liquid" constituent name would result in no entry for the "AN-103 Core 166, Segment 1 Liner Liquid"). A 79 by 29 matrix was produced. With this matrix, every analysis result for each sample description pertinent to waste layer density determination was available. Similar analyses were conducted for AN-104, AN-105, AW-101, and SY-103.

\subsubsection{Measurement Technique Effects}

Different measurement techniques on a given sample may yield different results with different expected accuracies. Thus, for example, the constituent liquid density, as computed from a measured sample volume and mass, may differ from the constituent specific gravity of the same sample. Layer density results for AN-103 are summarized in Table 3.1. Anomalous data points (e.g., Specific gravity less than or approaching that of water or greater than the bulk density) were discarded. The ball rheometer results are also listed. As expected, the standard deviation of the specific gravity measurement is less than that of the liquid density measurement, and significant anomalies are observable in densities computed from interstitial liquid (IL) and drainable liquid (DL) mass and volume measurements. Therefore, where both types of measurements are available, the specific gravity data is used instead of the density data.

There are significantly fewer liquid specific gravity measurements for the 2000 data as opposed to the 1996-1997 data. Additionally, the 2000 measurements are available for only two segments. The liquid density results from the Core 274 , as computed from the reported mass, $\mathrm{m}$, and volume, V, of interstitial liquid, IL, and drainable liquid, DL, are not physically plausible (see Table 3.1).

\subsubsection{Sample Handling Effects}

Sample handling may affect sample analysis. For layer density considerations, segment bulk measurements are not expected to be affected (i.e., the mass and volume of the bulk sample). However, for liquid segments or segment portions, some results may be significantly affected. For example, solids were observed in the numerous core segments taken from the supernatant liquid layer in AN-103, AW-101, and SY-103. Possible explanations include solids released from the crust as the core sampling is conducted, solids suspended by gas release activity, and precipitation of dissolved solids as sample temperature cools from in-tank to ex-tank conditions. 
Table 3.1. Layer Density Results for AN-103

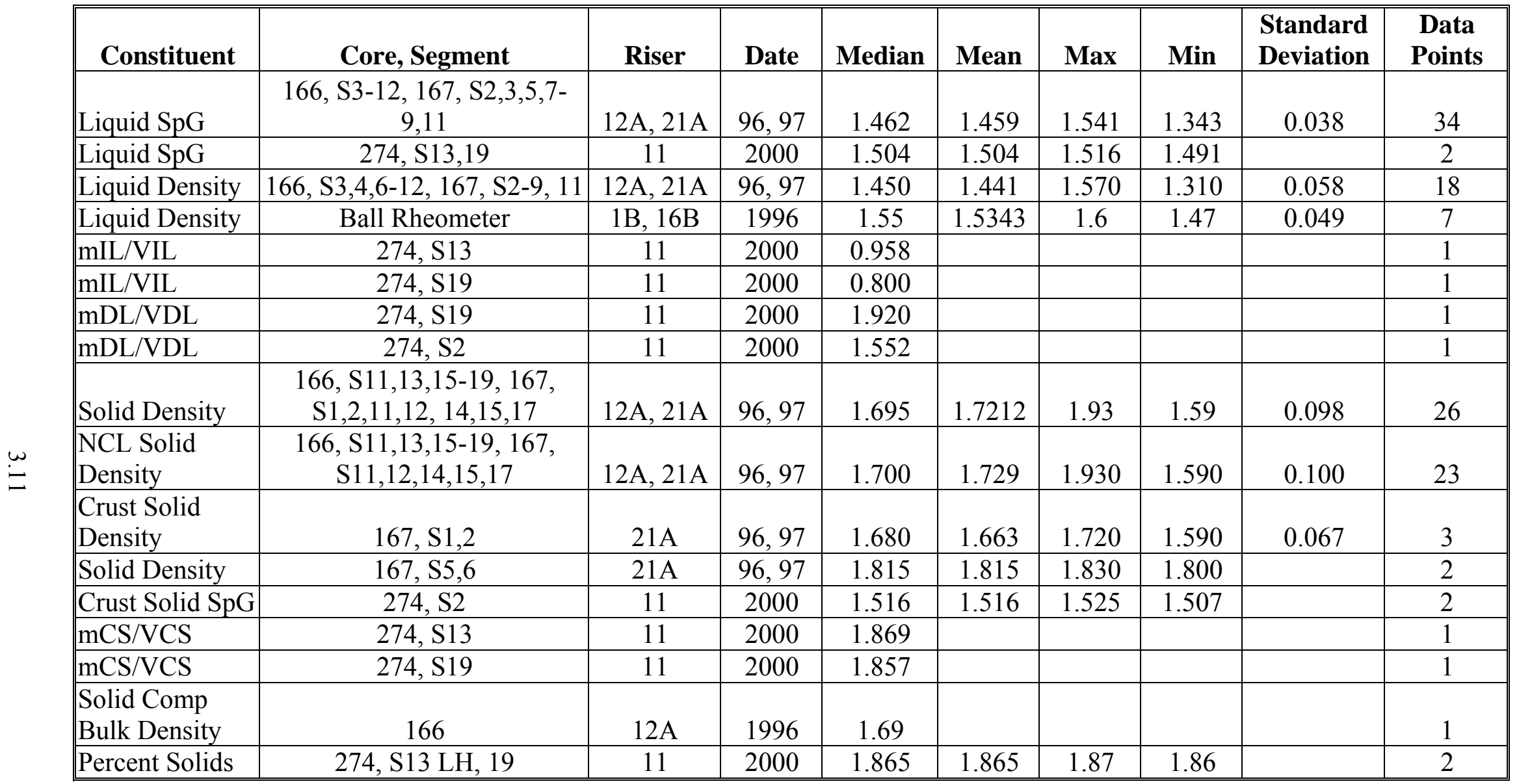


The possibility of a significant quantity of solids released from the crust seems implausible given that the drill string used in core sampling is intended to prevent much subsequent disturbance of an already-sampled elevation. Considering the effects of the initial disturbance may tell a different story, however. The volume of solids potentially initially released from the crust can be easily bounded on the lower end by approximating the sampler diameter, crust depth, gas fraction, and solid fraction. The volume of solids present in the liquid samples, $\mathrm{V}_{\mathrm{S}}$, may be estimated from

$$
\mathrm{V}_{\mathrm{S}}=\sum_{\mathrm{i}=1}^{\mathrm{n}} \frac{\mathrm{w}_{\mathrm{S}, \mathrm{i}} \rho_{\mathrm{B}} \mathrm{V}_{\mathrm{T}}}{\rho_{\mathrm{S}, \mathrm{L}}}
$$

where $\mathrm{n}$ is the number of liquid segments, $\mathrm{w}_{\mathrm{S}}$ is the measured mass fraction of solids (computed in Eq. 3.8), $\rho_{\mathrm{B}}$ is the bulk segment density (includes the liquid and solid components), $V_{\mathrm{T}}$ is the volume of the core sampler, and $\rho_{\mathrm{S}, \mathrm{L}}$ is the density of the solids in the liquid sample. The approximate lower-bound crust-release and core segment total volumes computed for AN-103 for Cores 166 and 167 are roughly equivalent. Although insufficient data are provided to compute solid fractions in the liquid segments of Core 274, it is noted that solids were present.

Core 166 was taken in AN-103 over a period of four days. Therefore, given that the available solids volume is similar to that from the liquid layer core segments, as discussed above, it is of interest to consider the settling time of potentially released solids. The fall velocity, $u$, of a spherical particle of diameter $d$ may be computed from Stokes law as

$$
\mathrm{u}=\frac{\mathrm{gd}^{2}}{18 \mu}\left(\rho_{\mathrm{P}}-\rho_{\mathrm{L}}\right)
$$

where $\mathrm{g}$ is the acceleration of gravity, $\mu$ is the liquid viscosity, and $\rho_{\mathrm{P}}$ is the particle density.

For representative AN-103 conditions with a $10-\mu \mathrm{m}$ particle and $\mu=0.002 \mathrm{~Pa} \mathrm{~s}, \rho_{\mathrm{P}}=$ $2,200 \mathrm{~kg} / \mathrm{m}^{3}$, and $\rho_{\mathrm{L}}=1,500 \mathrm{~kg} / \mathrm{m}^{3}$, the settling velocity is approximately $1.65 \mathrm{~m} / \mathrm{d}$. Thus approximately four days are required for the particles to reach the bottom of AN-103's approximately 6-m-deep liquid layer. The four days of sampling time included the sediment, so the sampler may have reached the bottom of the liquid layer before the solids. This observation is rendered meaningless, however, given the uncertainties in the waste rheology, particle size, and sampling rate as opposed to total time required. These results illustrate that it is possible for a disturbance of the crust to provide solids for the liquid samples.

The ball rheometer data for AN-103 may also support the ability of a crust disturbance to release solids into the liquid layer. The increasing liquid density with depth was attributed to waste disturbance, or crust layer disruption (Hedengren et al. 2000). However, the density gradient and the relatively rapid deployment of the ball rheometer compared with core sampling 
event time (up to months for the additional tanks with solids in the liquid core segments) indicates that the particle setting velocity may be so rapid that a crust disturbance would be unlikely to result in distribution of solids throughout the liquid core segments over the entire core sampling period. The mass fraction of solids in the liquid core segments was thus investigated. It suggests that a clear trend may support crust-solids release, while the lack of a trend or a relatively constant value may indicate solids precipitation or gas release events from the sediment mixing solids into the supernatant.

The mass fraction of solids in the liquid samples may be computed from the measured data by considering that the total mass is equal to the sum of the solid and liquid masses from which

$$
\mathrm{w}_{\mathrm{s}}=\frac{\mathrm{m}_{\mathrm{s}}}{\mathrm{m}_{\mathrm{s}}+\mathrm{m}_{\mathrm{L}}}
$$

where $\mathrm{m}_{\mathrm{S}}$ and $\mathrm{m}_{\mathrm{L}}$ are the measured solid and liquid mass of a segment, respectively. Results for Cores 166 and 167 are shown in Figure 3.6 (no data are available for Core 274). The potential uncertainty in the data may exacerbate identification of similarities or trends. Thus, especially for Core 166, the relatively minimal scatter and lack of clear trends may suggest that either gas release events or solid precipitation from the sample due to temperature changes result in the presence of the solids.

The potential for gas release activity being the cause of the solids is easily investigated by comparing the date of the core sampling to gas release event history. As may be seen in Section 3.2.7, AN-103 had no recorded gas release events in 1996, while the closest event to

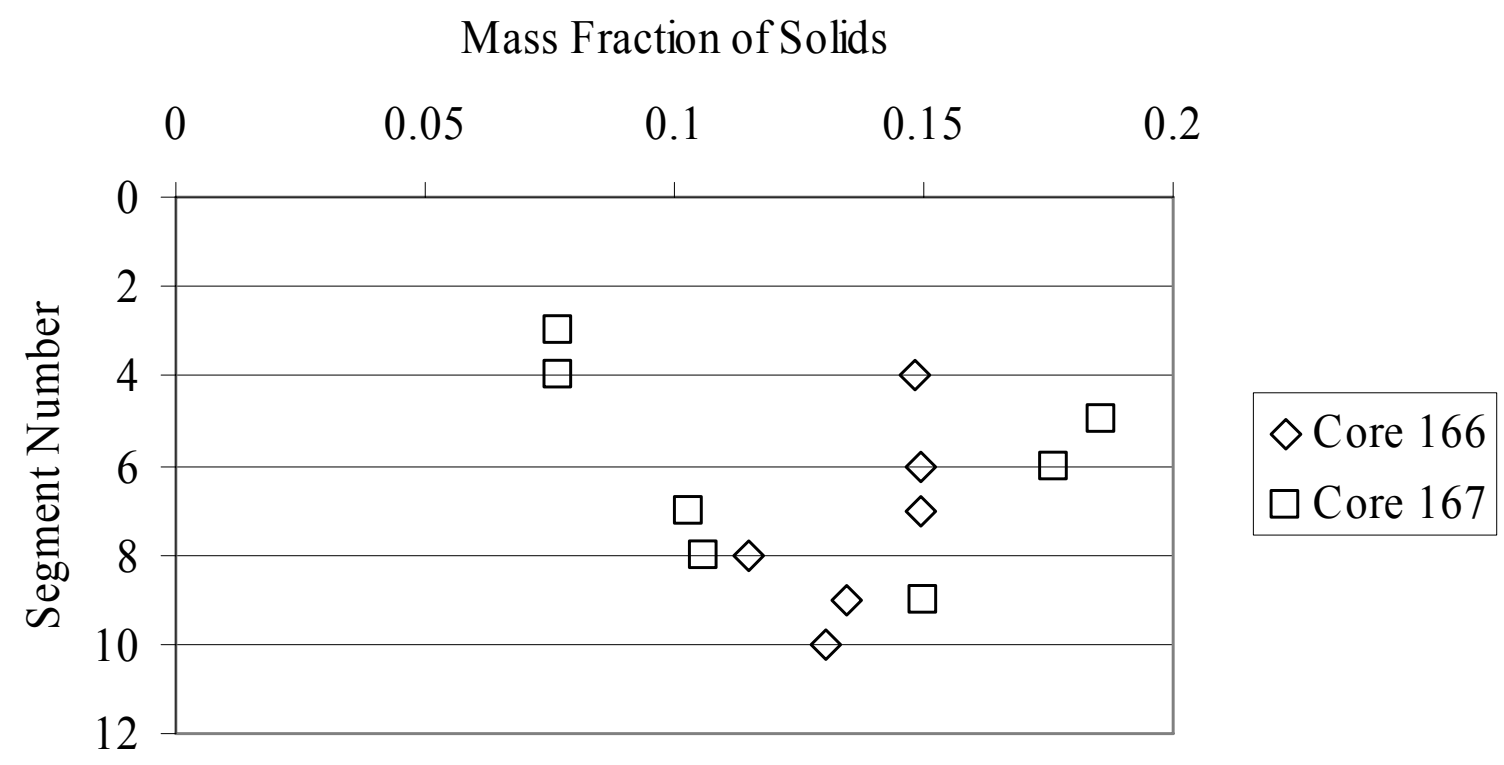

Figure 3.6. Mass Fraction of Solids in Liquid Core Segments from AN-103 
Core 274 (taken in February 2000) was in December 1999. Thus gas release events are not expected to have contributed to the presence of solids in the liquid, rendering solids precipitation the only probable alternative.

Additional insight into the source of solids in the liquid segments may be gained by considering data from tanks AW-101 and SY-103, which both contained solids in some of their liquid core segments. However, unlike AN-103, AW-101 had a subsequent waste core (initiated within two weeks of the previous core) without solids present in a number of the liquid segments. This observation may argue against crust penetration causing the solids. The mass fraction of solids in the liquid samples for AW-101 and SY-103 is shown in Figure 3.7. As with $\mathrm{AN}-103$, there is no definitive indication that the solids are from crust disturbance.

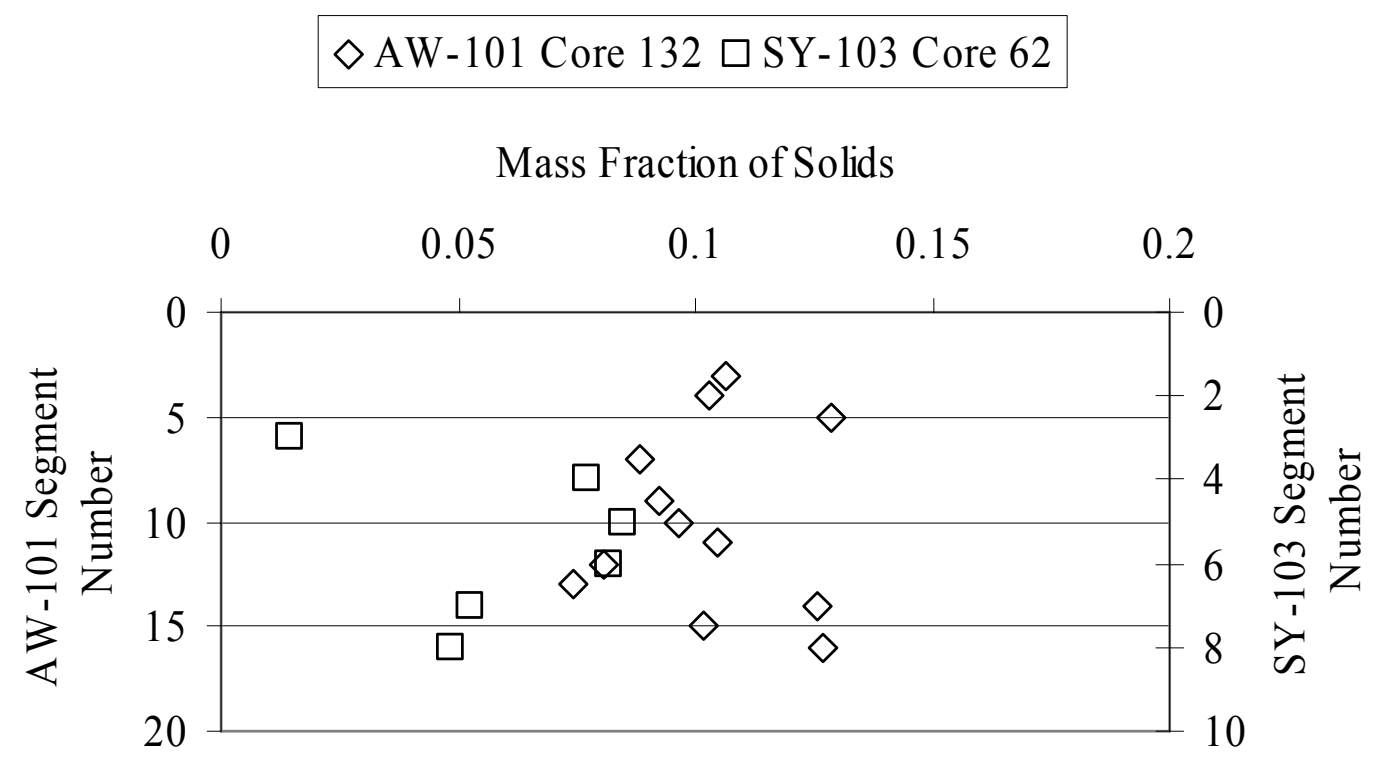

Figure 3.7. Mass Fraction of Solids in Liquid Segments from AW-101 and SY-103

In AW-101, the mass fraction profile is relatively uniform (Figure 3.7). Additionally, Cores 132 and 139, taken within the same three-month period, show distinct liquid density differences. The liquid segment result for Core 132 is $1.401 \mathrm{~g} / \mathrm{mL}$ (without contribution from the contained solids), and $1.472 \mathrm{~g} / \mathrm{mL}$ for Core 139 (see Appendix B). Most of the liquid segments from Core 132 contained solids, while most of those from Core 139 did not. These considerations argue strongly in favor of the solids being the result of precipitation. That is, given the convection present in the supernatant (and the relative equilibrium condition of the tank), it is not expected that significant liquid density gradients exist. Thus, the higher density of the solids-free liquid of Core 139 would suggest that the lower result of solids-containing Core 132 is due to precipitation of those solids. 
Gas release data are not available for the pertinent SY-103 waste core. For AW-101, however, gas release events occurred approximately four weeks, three weeks, and one week before the sampling event. Additionally, an event was recorded on the day the core sampling was initiated. However, as noted above, the uniform mass fraction of solids and the distinct density differences in the cores support solids precipitation.

The phenomenon of solids precipitation from waste samples has been investigated experimentally. For example, Core 257 was taken in March of 1999 while SY-101 was being mixed regularly with its mixer pump. Liquid decanted from centrifuged liquid (as a result of the centrifugation, the decanted sample was not expected to nor observed to contain solids initially) was observed to contain solids after the samples were cooled.(a) Conversely, no solids were observed to precipitate from supernatant liquid samples of $\mathrm{AN}-104$ liquid (nominally $40^{\circ} \mathrm{C}$ intank) after two weeks at $25^{\circ}, 45^{\circ}$, and $65^{\circ} \mathrm{C}$ (Herting 1998).

These observations suggest that the most plausible explanation for solids in supernatant liquid core segments is solids precipitation as the sample is cooled ex-tank. Thus, these solids must be accounted for in determining the in situ liquid density. From Eq. (3.8), we know the mass fraction of the solids in the sample. Further, the summation of the total mass from the solid and liquid portions yields

$$
\rho_{\mathrm{L}}=\frac{1}{\frac{\mathrm{w}_{\mathrm{S}}}{\rho_{\mathrm{PS}}}+\frac{1-\mathrm{w}_{\mathrm{S}}}{\rho_{\mathrm{LM}}}}
$$

where $\rho_{\text {PS }}$ is the density of the precipitated solids, and $\rho_{\mathrm{LM}}$ is the density of the liquid portion of the segment. The precipitated solids, like the sediment or crust core segments, include interstitial liquid. Thus, while consideration of the waste chemistry may indicate the precipitation analyte, the crystal density is not expected to be representative of the precipitate. The sediment layers in the specific tanks of concern contain insoluble solids as well as various soluble species. Thus, the precipitate may not be equivalent in density to the sediment. Further, like attempts to compute a bulk density accounting for interstitial liquid density and solid crystal density, the undissolved solid fraction in the precipitate is unknown and may be different from the sediment or crust. Thus, the most plausible option for precipitate density is sample measurement. For AN-103, two measurements were reported on solids from Segments 5 and 6, Core 167. The resulting liquid specific gravity from 24 data points covering 13 core segments available for Cores 166 and 167 with the solids added is $1.502 \mathrm{~g} / \mathrm{mL}$.

(a) Person JC to NW Kirch. 1999. Dilution Studies of Tank 241-SY-101 Waste. Preliminary Report. Correspondence Number 82100-99-015, Numatec Hanford Corporation, Richland, Washington. 


\subsubsection{Hot and Cold Tank Liquid Density Data}

The similarity in liquid density in the hot and cold tank states in AN-103g/mL) (Table 3.1) agrees with equilibrium chemical modeling for AN-103 liquid in contact with undissolved solids. (a) Core 274 had solids in its liquid segments, so the cold data may not be representative (insufficient data are available to compute the effect of the solids).

Significant discrepancies exist in the hot and cold liquid density data for AN-104, AN-105, and AW-101 (see Appendix B). The cold tank liquid density results for AN-104, AN-105, and AW-101 are approximately 0.09 to $0.04 \mathrm{~g} / \mathrm{mL}$ higher than hot tank results. Like AN-103, the results for cold tanks are significantly less than those for hot tanks, and there are many nonphysical results (e.g., density less than that of water or greater than that of sediment). The liquid density values for the cold tanks are for drainable liquid from core segments in either the crust or sediment layers. Thus, the density of the interstitial liquid is different than that of the supernatant. For AN-105, the solid-segment drainable liquid median density (six data points for three core segments) is equivalent to the supernatant liquid segment median result. The two measurements of a single segment in AN-104 have a median approximately $0.08 \mathrm{~g} / \mathrm{mL}$ greater than the bulk liquid. However, the drainable liquid results from the crust segment are approximately $0.2 \mathrm{~g} / \mathrm{mL}$ less than the supernatant liquid. Drainable liquid results for the crust segment in AN-105 are in agreement with the supernatant liquid. Though the elevated results may be caused by sample location (supernatant liquid or solids-containing layer), based on the extremely limited AN-104 result, the physical reality for the supernatant layer of the liquid density discrepancy between the hot and cold tanks is further considered.

The primary dissolved-solid constituents of the liquid become less soluble with decreased temperature, suggesting that the liquid density will decrease with cooling. Available tank waste data support this (Figure 3.8). However, these data are for liquid in contact with solids during temperature changes. Thus, the increase in liquid density with heating is expected because of solubility variation and the subsequent dissolution of solids. In the absence of solids contact, the heated fluid, like water, may become less dense. In-tank data irrefutably show that the liquid layers are convective. Thus, with heating on the bottom of the layer and cooling on the top and sides, the liquid density can only plausibly behave like the base liquid, water.

The laboratory data depicted in Figure 3.8 support minimal density changes over temperature differences much larger than the $3{ }^{\circ} \mathrm{C}$ indicated for the distinction between the hot and cold tanks. Finally, if the in-tank cooling did cause an increase of approximately $0.09 \mathrm{~g} / \mathrm{mL}$ (without dissolution; solubility effects make cooler liquid less dense), the required tank volume change of nominally $150 \mathrm{~m}^{3}$ would have been readily apparent in the waste surface level ( $0.4 \mathrm{~m}$ or $\left.14 \mathrm{in}\right)$. Level changes of this magnitude have not occurred over the temperature decrease interval (Hedengren et al. 2000).

(a) Personal communication with LA Mahoney, PNNL. 


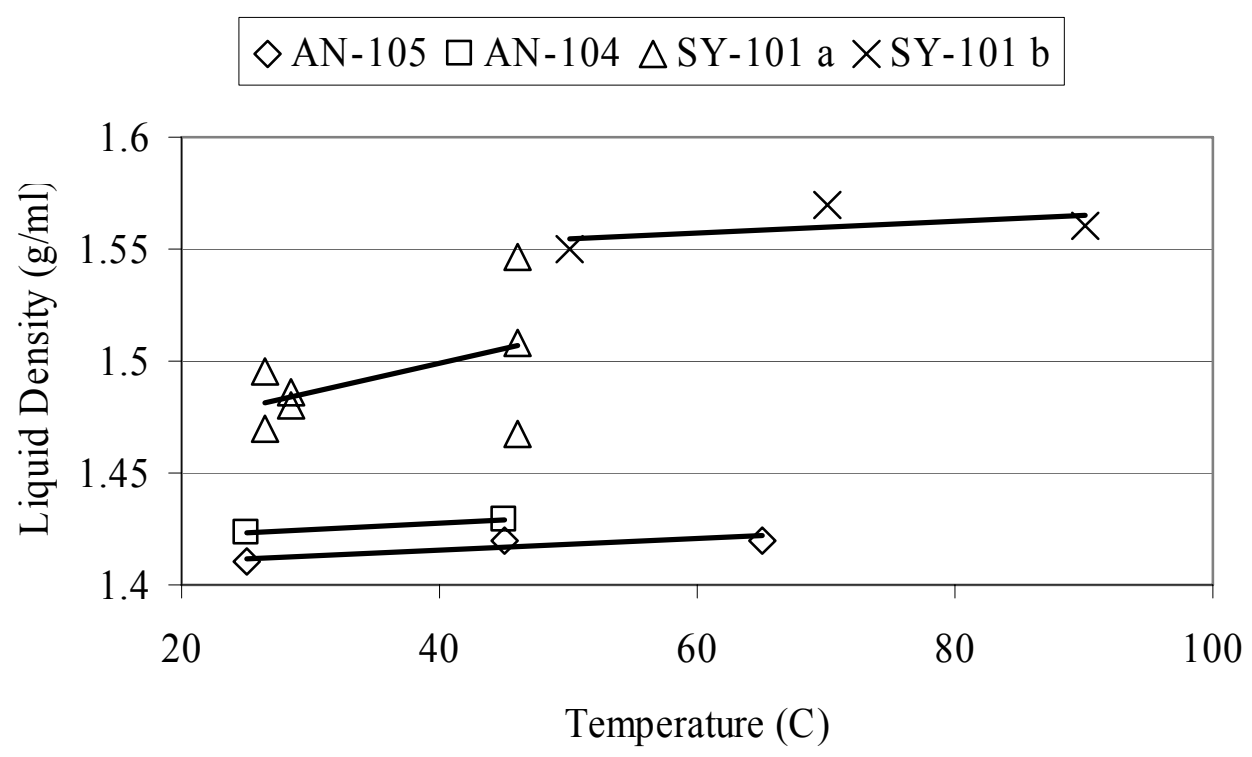

Figure 3.8. $\quad$ Liquid Density as a Function of Temperature. AN-105 (Herting 1997), AN-104 (Herting 1998), SY-101, (a) (Tingey et al. 1994).

\subsubsection{Liquid Density Summary}

The liquid density data for the cold tank states is not used, and the determined supernatant liquid density distributions for the hot tanks are listed in Table 3.2. Like AN-103, solids present in liquid samples are included for AW-101 and SY-103. Ball rheometer results are included in AN-104, AN-105, and AW-101 and neglected in AN-103 and SY-103 due to the expectation that the results were affected by waste disturbance (Hedengren et al. 2000).

Table 3.2. Supernatant Liquid Density Distributions (g/mL)

\begin{tabular}{|c|c|c|c|c|c|c||}
\hline \hline Tank & Median & Mean & Max & Min & $\begin{array}{c}\text { Standard } \\
\text { Deviation }\end{array}$ & $\begin{array}{c}\text { Data } \\
\text { Points }\end{array}$ \\
\hline AN-103 & 1.502 & 1.497 & 1.559 & 1.390 & 0.034 & 24 \\
\hline AN-104 & 1.408 & 1.403 & 1.5 & 1.339 & 0.034 & 48 \\
\hline AN-105 & 1.410 & 1.417 & 1.534 & 1.33 & 0.046 & 59 \\
\hline AW-101 & 1.439 & 1.443 & 1.524 & 1.37 & 0.039 & 67 \\
\hline SY-103 & 1.472 & 1.474 & 1.529 & 1.352 & 0.046 & 11 \\
\hline
\end{tabular}

(a) Person JC to NW Kirch. 1999. Dilution Studies of Tank 241-SY-101 Waste. Preliminary Report. Correspondence Number 82100-99-015, Numatec Hanford Corporation, Richland, Washington. 


\subsubsection{Sediment Gas Content Effect}

The sediment density is relatively unambiguous - the bulk density of the sediment layer segments - until one considers gas retention in this layer. However, the potential for gas content to affect the reported sediment segment density is easily investigated by considering the effect the gas would have on a density measurement. Assuming that the sediment layer is relatively homogeneous, as indicated by core segment chemical analyses and BDGRE behavior, a segment with higher gas content will have lower bulk density than a segment with less gas content.

From Hedengren et al. (2000) the gas fraction profiles in the sediments are either approx. imately linear or parabolic. That is, the gas fraction either increases linearly from the top to the bottom of the sediment or is at a minimum at the top and bottom of the layer and at a maximum roughly around the midpoint of the layer. Thus, if the analyzed samples still contained gas, the density should be at a maximum at the top or at the edges of the layer and at a minimum either at the bottom or at the middle. In fact, the measured densities appear to follow the gas fraction profiles (Figures 3.9 through 3.12), indicating that the in situ retained gas displaced liquid (data not available for SY-103). Additionally, comparison of available centrifuged solid density data for AN-104 (Herting 1998), AN-105 (Herting 1997), AW-101 (Herting et al. 1999), SY-101(a) (Tingey et al. 1994), and SY-103(b) to sediment density values from Hedengren et al. (2000) and results of the current analysis shows a median increase of approximately $4 \%$ for the centrifuged solid data. Thus, gas is not expected to have affected the sediment density results.

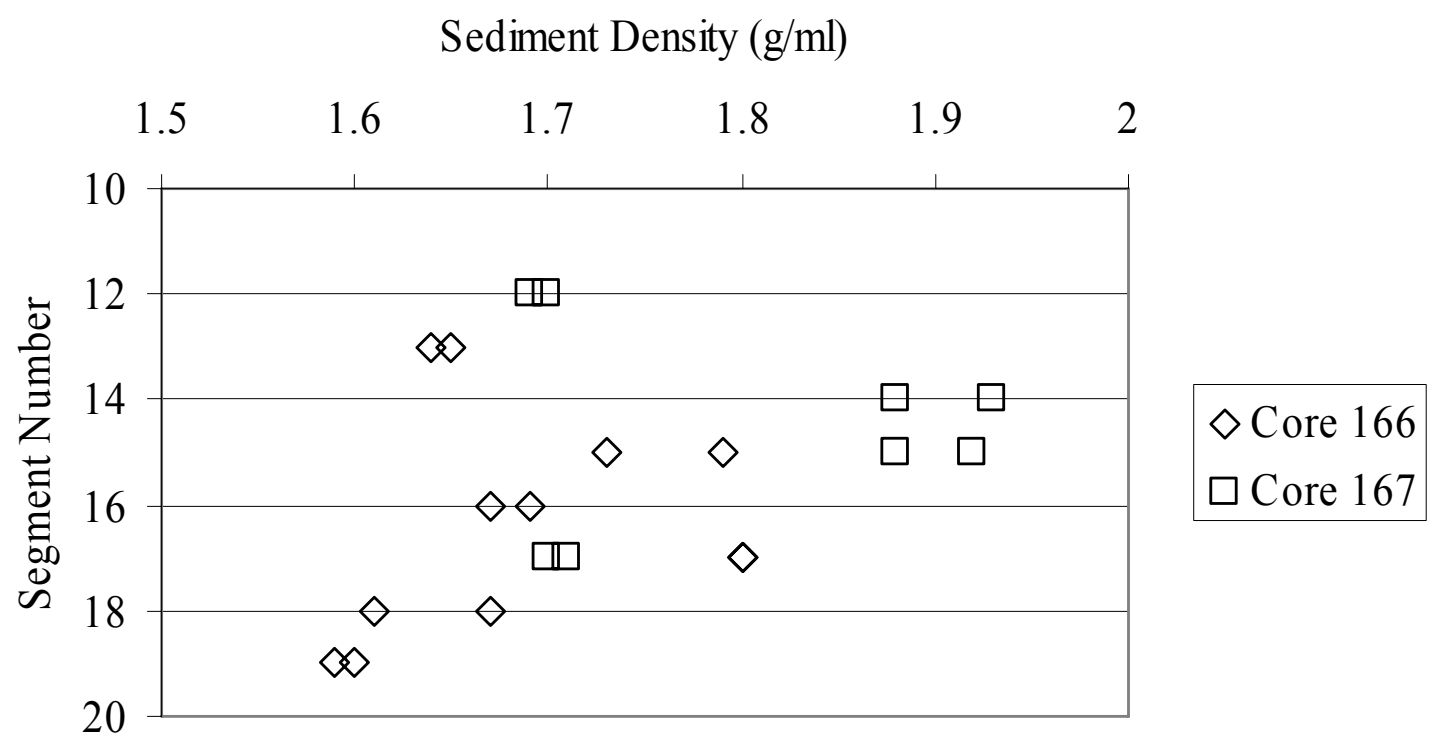

Figure 3.9. Sediment Density in Sediment Core Segments from AN-103

(a) Person JC to NW Kirch. 1999. Dilution Studies of Tank 241-SY-101 Waste. Preliminary Report. Correspondence Number 82100-99-015, Numatec Hanford Corporation, Richland, Washington.

(b) Bredt BR, JD Hudson, and JM Tingey. 1995. Effects of Dilution on the Physical, Rheological and Chemical Properties of Tank 241-SY-103 Waste. PNL MIT 092995, Pacific Northwest Laboratory, Richland, Washington. 


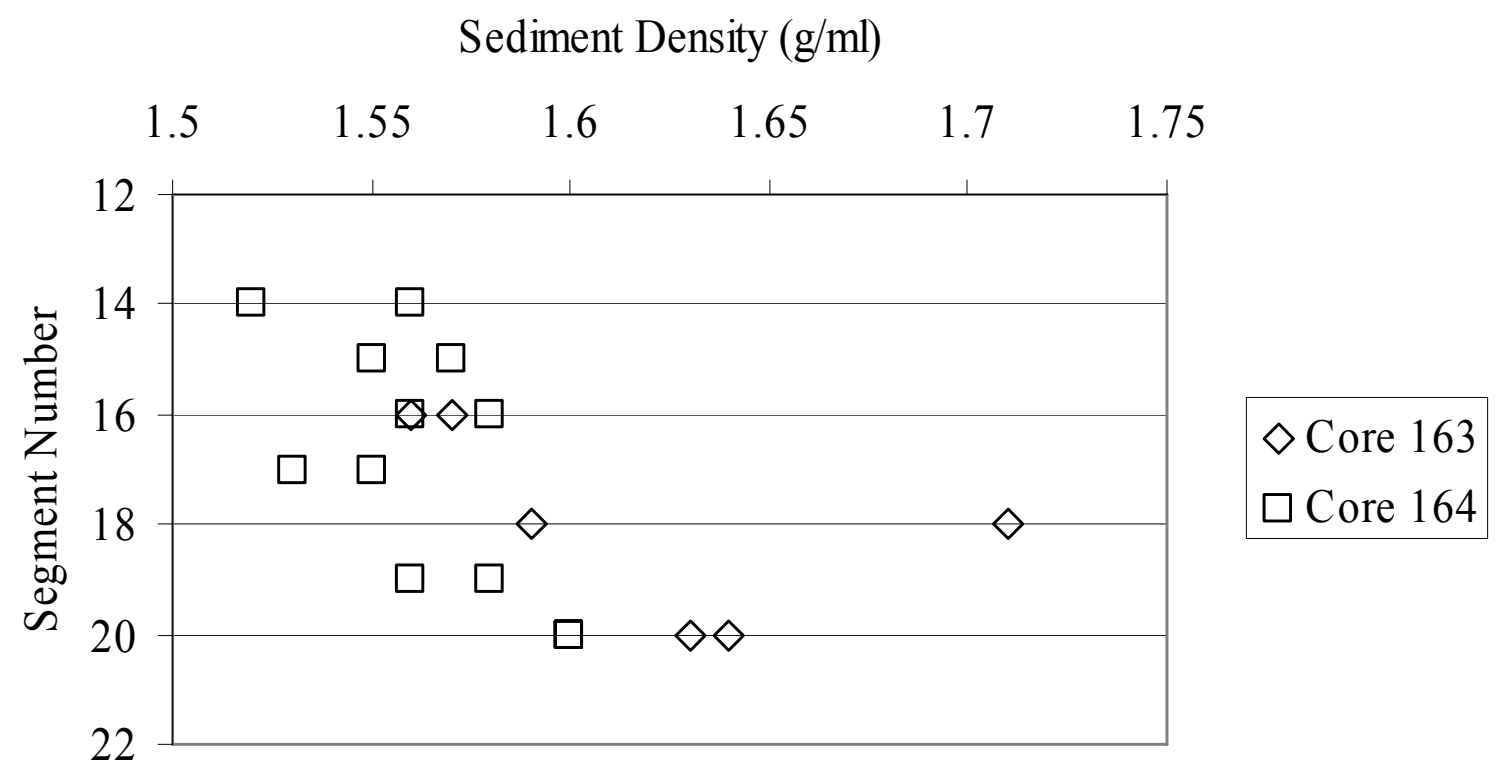

Figure 3.10. Sediment Density in Sediment Core Segments from AN-104

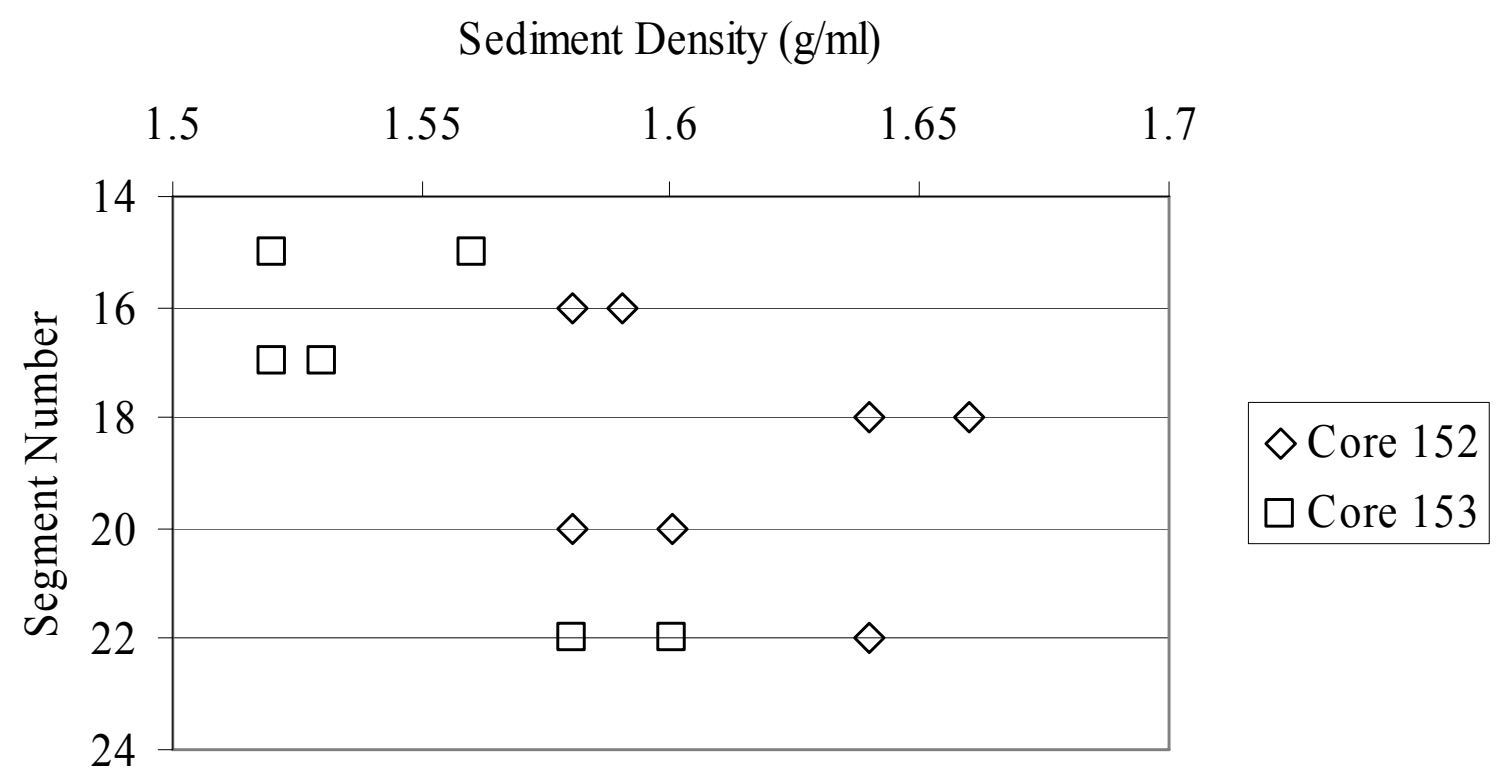

Figure 3.11. Sediment Density in Sediment Core Segments from AN-105 
Sediment Density $(\mathrm{g} / \mathrm{ml})$

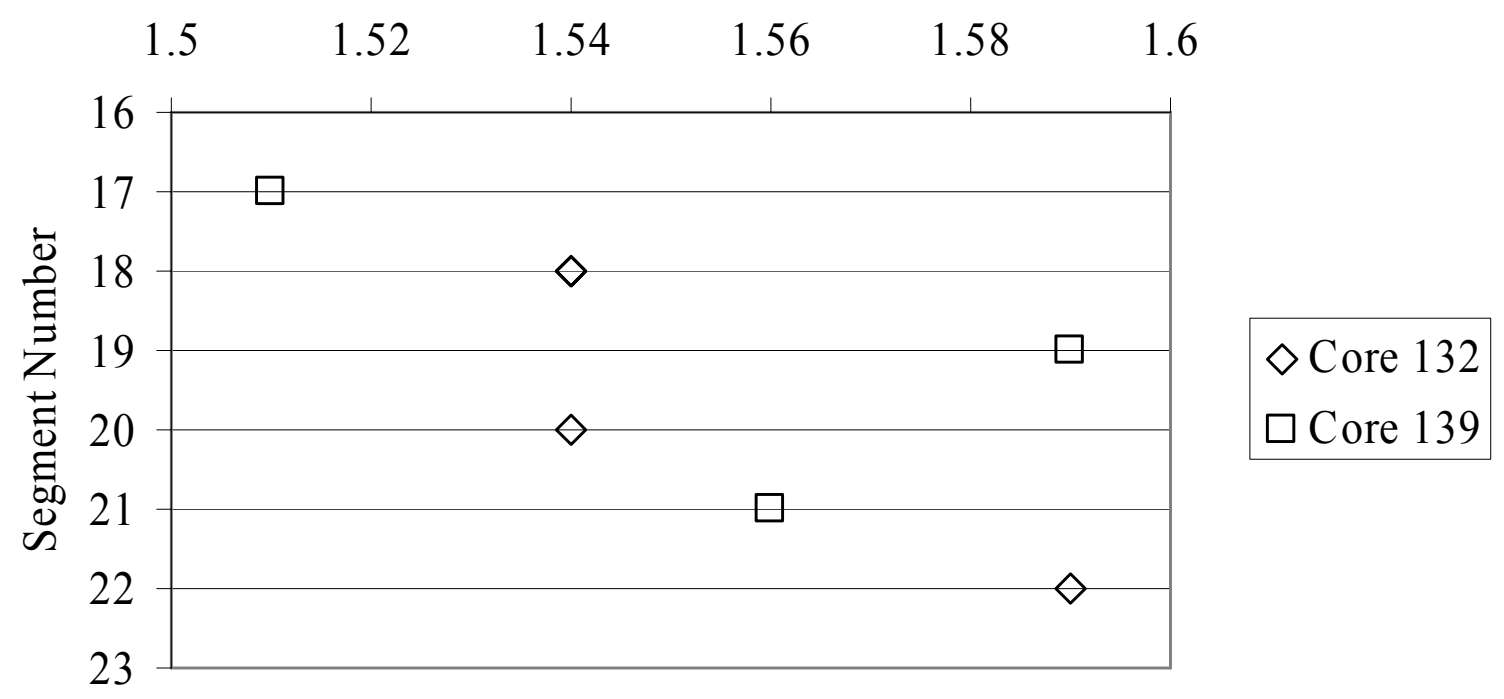

Figure 3.12. Sediment Density in Sediment Core Segments from AW-101

\subsubsection{Sediment Density Summary}

The determined sediment density results are listed in Table 3.3. Sediment segment results with drainable liquid were not included (this exclusion affected less than five measurements in any given tank).

Table 3.3. Sediment Density Distributions ( $\mathrm{g} / \mathrm{mL})$

\begin{tabular}{|c|c|c|c|c|c|c|}
\hline Tank & Median & Mean & Max & Min & $\begin{array}{c}\text { Standard } \\
\text { Deviation }\end{array}$ & $\begin{array}{c}\text { Data } \\
\text { Points }\end{array}$ \\
\hline AN-103 & 1.700 & 1.733 & 1.930 & 1.590 & 0.106 & 20 \\
\hline AN-104 & 1.570 & 1.578 & 1.710 & 1.520 & 0.045 & 19 \\
\hline AN-105 & 1.580 & 1.585 & 1.660 & 1.520 & 0.045 & 13 \\
\hline AW-101 & 1.575 & 1.570 & 1.600 & 1.540 & 0.027 & 6 \\
\hline SY-103 & 1.595 & 1.592 & 1.634 & 1.510 & 0.040 & 8 \\
\hline
\end{tabular}

\subsubsection{Liquid and Sediment Density Correlation}

Allowing the supernatant and sediment densities to vary indiscriminately and independently within their respective distributions will produce non-physical combinations. These nonphysical results may be illustrated indirectly through gas retention dictated by neutral buoyancy and controlled directly by solid/liquid fractions in the wastes. 
The tanks in question do indeed retain a quantity of gas and experience buoyant displacements. Therefore, from Eq. (1.1), sediment and liquid density combinations that produce neutral buoyant gas fractions that stray significantly from an expected value (that computed from the median densities) may not be meaningful. To illustrate, a low neutral buoyancy gas fraction would potentially preclude the observed gas release volumes or retained gas volume estimates from in situ measurements or waste surface level histories, while a high neutral buoyancy gas fraction would potentially exceed the estimated gas retention quantities.

Therefore, in addition to the obvious constraint that the supernatant liquid is less dense than the sediment, the sediment density must be correlated with the liquid density. It is assumed that the interstitial liquid present in the sediment is essentially identical to the supernatant liquid. Thus the sediment density, with consideration of the layer properties, may be computed directly from the supernatant density. The in situ sediment is a solid-liquid-gas matrix. In its degassed state, the total mass is the sum of the liquid and solid masses. Similar to Eq. (3.9), the bulk sediment density may then be expressed as

$$
\rho_{\mathrm{NCL}}=\phi \rho_{\mathrm{S}}+(1-\phi) \rho_{\mathrm{L}}
$$

where $\phi$ is the solid volume fraction and $\rho_{\mathrm{S}}$ is the undissolved solid density. We have chosen the solid volume fraction for each tank as that computed with the respective median sediment and liquid densities. That is,

$$
\phi=\frac{\rho_{\mathrm{NCLmed}}-\rho_{\mathrm{Lmed}}}{\rho_{\mathrm{S}}-\rho_{\mathrm{Lmed}}}
$$

A representative undissolved solid density of $2.3 \mathrm{~g} / \mathrm{mL}$ is used for the analysis. With the median liquid and sediment density values from Tables 3.2 and 3.3, the computed solid volume fractions are 0.25 for $\mathrm{AN}-103,0.18$ for $\mathrm{AN}-104,0.19$ for $\mathrm{AN}-105,0.16$ for $\mathrm{AW}-101$, and 0.15 for SY-103. For SY-101, with median liquid and sediment density values of $1.49 \mathrm{~g} / \mathrm{mL}$ (Rassat et al. 2000) and $1.7 \mathrm{~g} / \mathrm{mL}$ (Meyer et al. 1997), respectively, the solid volume fraction is 0.26 . The validity of using the methodology of Eq. (3.10) and (3.11) is discussed below.

Several methods have been used to estimate the undissolved solids density of Hanford waste. Chemical analyses and simulations provide solid species information; the undissolved solids density may be computed by combining the species using handbook density values. This method has uncertainties due to the underlying analyses as well as not accounting for possible agglomeration and/or flocculation. Analyses of dilution studies based on conservation of mass have also been used (Rassat et al. 2000).

An additional approach is to use water content data from the bulk solids and liquid. A similar method is used to determine sample solid/liquid content. As presented in Onishi et al. (2002); however, this solids density methodology is extremely sensitive to the relatively high 
uncertainty (compared with the other parameters) in the measured water content in the settled solids and may produce erroneous results (as determined by comparison to possible species without agglomeration effects).

The combined solid crystal densities for the identified solid species for AN-104, AN-105, and AW-101 are 2.3, 2.4, and $2.1 \mathrm{~g} / \mathrm{mL}$ (Herting 1997, 1998, 1999). Equilibrium chemical modeling for AN-103, AN-104, AN-105, AW-101, and SY-103 indicates $2.2 \mathrm{~g} / \mathrm{mL}$ for all the tanks except AN-104, which has an undissolved solids density of $2.4 \mathrm{~g} / \mathrm{mL}$. (a) Rassat et al. (2000) determined an undissolved solid density of $2.2 \mathrm{~g} / \mathrm{mL}$ for the bulk solids (possibly including agglomeration or other effects). As suggested above, the chemistry estimates may be considered to be an upper bound due to possible agglomeration or associated effects, which have been estimated to cause up to $15 \%$ reduction in the combined solid crystal density. (b) Thus, given the relative uncertainties of the methodologies, $2.3 \mathrm{~g} / \mathrm{mL}$ is considered a reasonably representative value for the undissolved solid density for the tanks of concern. In application, however, the true test of this value is essentially synonymous with the test of a fixed solid volume fraction via Eq. (3.10) for the sediment density; determine whether the methodology, when applied with the liquid density distribution, approximates the sediment density distributions of Table 3.3.

Comparisons of the sediment density computed with Eq. (3.10) and the sediment density distributions of Table 3.3 are favorable, as shown in Table 3.4. Visual comparisons may be made of these data in Appendix C. The larger standard deviations of the data are not unexpected given the apparent effect of gas retention displacing liquid from the sediment. This effect would expand the sediment density range, suggesting an associated large variance in the solids volume fraction. AN-103 is the most extreme example, and this result is not unexpected given its larger retained gas volume (Hedengren et al. 2000). In addition, extreme values are not representative of the average bulk of the sediment. Thus, we believe that the results of Eq. (3.10) are a reasonable representation of the variability of the average bulk degassed sediment density.

An example of the effect of this methodology on the neutral buoyant gas fraction is shown for AN-103 in Figure 3.13. Computation of the neutral buoyant gas fraction indiscriminately using the data density distributions yields results ranging from approximately 0.02 to 0.27 . These results under-predict the reported gas content in the sediment layer from Hedengren et al. (2000) by a factor of 6 and over-predict by a factor of 2.5. Conversely, with the sediment density computed via Eq. (3.11), the neutral buoyant gas fraction ranges from approximately 0.10 to 0.14 , under- and over-predicting by factors of 1.1 and 1.3 , respectively.

(a) Personal communication with LA Mahoney, PNNL.

(b) Wells BE. 2004. Evaluation of Waste Data for Rheological Models Used in Waste Pipeline Transfer

Assessment. PNNL Letter Report TWS05.001, Pacific Northwest National Laboratory, Richland, Washington. 
Table 3.4. Comparison of Sediment Density Distributions $(\mathrm{g} / \mathrm{mL})$

\begin{tabular}{|c|c|c|c|c|c|c||}
\hline \multirow{2}{*}{ Tank } & Source & Median & Mean & Max & Min & $\begin{array}{c}\text { Standard } \\
\text { Deviation }\end{array}$ \\
\hline \multirow{2}{*}{ AN-103 } & Data & 1.700 & 1.733 & 1.930 & 1.590 & 0.106 \\
\cline { 2 - 7 } & Eq. (3.2.5) & 1.701 & 1.696 & 1.743 & 1.617 & 0.027 \\
\hline \multirow{2}{*}{ AN-104 } & Data & 1.570 & 1.578 & 1.710 & 1.520 & 0.045 \\
\cline { 2 - 7 } & Eq. (3.2.5) & 1.570 & 1.570 & 1.645 & 1.513 & 0.021 \\
\hline \multirow{2}{*}{ AN-105 } & Data & 1.580 & 1.585 & 1.660 & 1.520 & 0.045 \\
\cline { 2 - 7 } & Eq. (3.2.5) & 1.581 & 1.585 & 1.680 & 1.516 & 0.036 \\
\hline \multirow{2}{*}{ AW-101 } & Data & 1.575 & 1.570 & 1.600 & 1.540 & 0.027 \\
\cline { 2 - 7 } & Eq. (3.2.5) & 1.575 & 1.578 & 1.646 & 1.517 & 0.019 \\
\hline \multirow{2}{*}{ SY-103 } & Data & 1.595 & 1.592 & 1.634 & 1.510 & 0.040 \\
\cline { 2 - 7 } & Eq. (3.2.5) & 1.595 & 1.596 & 1.644 & 1.509 & 0.021 \\
\hline
\end{tabular}

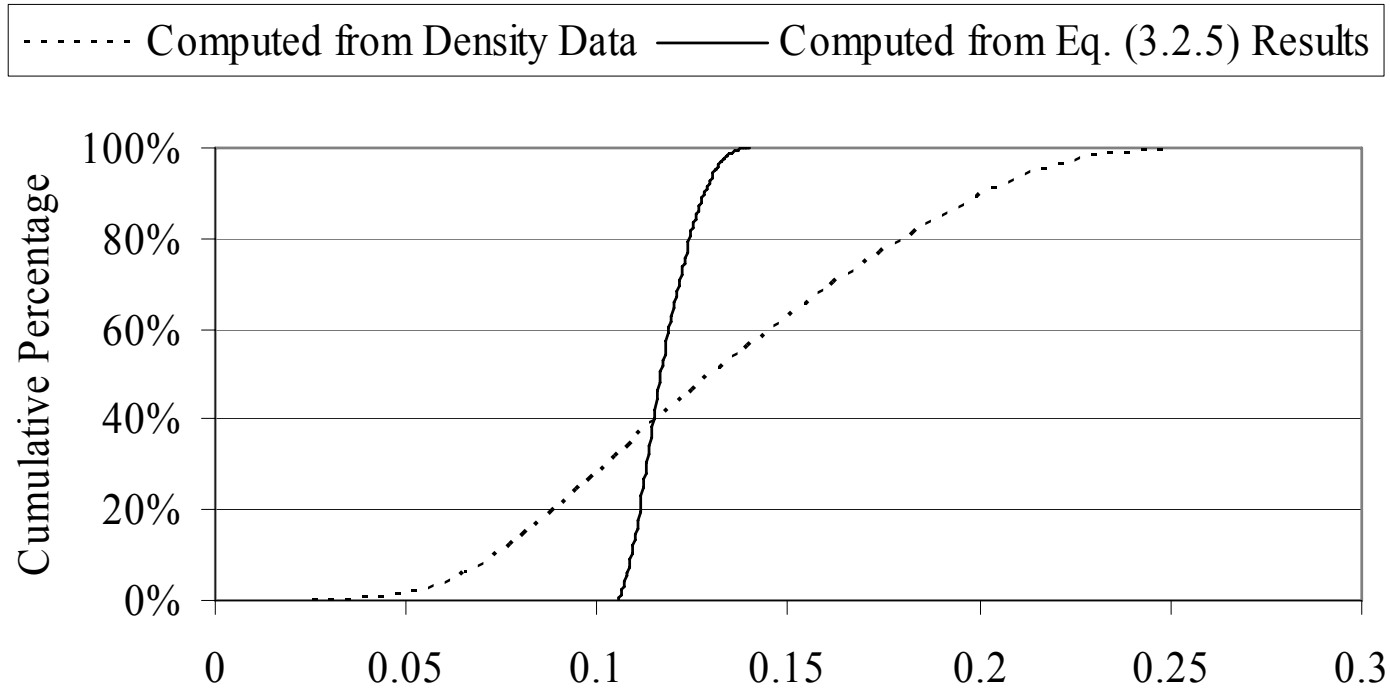

Neutral Buoyant Gas Fraction

Figure 3.13. Example Neutral Buoyant Gas Fractions in AN-103

\subsubsection{Waste Surface Level and Headspace Volume}

The surface level is used to compute local pressure for the buoyancy ratio and to define the headspace volume to form the $(\mathrm{BR}-1)\left[\mathrm{H}_{2}\right]_{\text {gas }} / \mathrm{V}_{\mathrm{HS}}$ quantity for the model and flammability criteria evaluation. The waste level for the buoyancy ratio calculation was computed as the average of the daily Enraf readings during the hot and cold periods of each tank. The implicit assumption in this calculation is that the single-point measurement represents the elevation of the entire waste surface. While significant uncertainty is involved, its effect on hydrostatic pressure is small, and 
variation in the headspace is negligible compared to the potential uncertainty of the measured hydrogen concentration. No uncertainty distribution is applied to waste level.

The headspace was computed separately for each BDGRE from the waste level recorded on the day it occurred. In SY-101, where level drops were significant, the first measurement after the initial large drop was used. The headspace calculation included all the details of the tank geometry (Barker 2003). The average headspace volume calculated for all BDGREs is used to calculate $(\mathrm{BR}-1)\left[\mathrm{H}_{2}\right]_{\text {gas }} / \mathrm{V}_{\mathrm{HS}}$ for each hot and cold tank in the model.

\subsubsection{Layer Depths}

As depicted in Figure 1.1, the tanks under consideration have a crust-liquid-sediment layer configuration. Based on extensive analysis of the large and gaseous crust in SY-101 during mixer pump operation (Rassat et al. 2000), limited freeboard is expected for crust layers. Thus, for this analysis, the non-sediment layers serve only to occupy tank headspace and apply hydrostatic pressure to the retained gas. The crust is treated as part of the liquid layer. This total liquid layer depth may be approximated from the total waste depth minus the sediment depth.

The median sediment depth and uncertainty range for SY-101 for the time of the documented BDGREs (1989-1993) is derived from the reported sediment depth inferred from temperature profiles in Antoniak (1993). The median sediment depth, $5.33 \mathrm{~m}$, is normally distributed with a standard deviation of $0.64 \mathrm{~m}$. Sediment depth data for the remaining tanks are determined from three different sources: 1) ball rheometer data, 2) core sample data, and 3) validation probe temperature measurements in the multifunction instrument trees. As presented in Appendix D, the most accurate measurements are thought to be from the ball rheometer, followed by the validation probes; the core sample data are judged to be much less accurate.

Ball rheometer data are taken from Hedengren et al. (2000) and summarized in Table 3.4. Sediment depth data are inferred from core sample profiles available from TWINS(a) for AN-103, AN-104, AN-105, AW-101, and SY-103; the estimates are presented in Table 3.5.

Temperature validation probe data are taken from Hedengren et al. (2000) and documents 2E-99-00124/P, 2E-99-00125/P, 2E-99-00126/P, 2E-99-00127/P, 2E-00-00675/P, 2W-0000736/P, 2E-00-01245/P, 2E-00-01397/P, 2W-00-01236/P, 2W-00-01397/P, and 2W-01000202/P. As discussed in Hedengren et al. (2000), the layer interface is visually identified by determining the intersection of the uniform temperature profile of the convective liquid and the parabolic temperature profile of the sediment. Example temperature profiles of the liquidsediment interface region for AN-103 are provided in Figure 3.14. The sediment layer depth is determined as $3.64 \mathrm{~m}$ for the 1996 validation probe and $3.71 \mathrm{~m}$ for the 1999 readings. The determined values for each tank are listed in Table 3.4.

(a) TWINS, Tank Waste Information Network System, http://twins.pnl.gov/drawings/hanford_drawings.asp. 
Table 3.5. Sediment Layer Depth Data

\begin{tabular}{|c|c|c|c|}
\hline \multirow[b]{2}{*}{ Tank } & \multicolumn{3}{|c|}{ Sediment Depth (m) } \\
\hline & $\begin{array}{c}\text { Ball Rheometer } \\
\text { [mo/yr, riser] }\end{array}$ & $\begin{array}{l}\text { Core Samples } \\
\text { [mo/yr, riser] }\end{array}$ & $\begin{array}{l}\text { MIT Validation Probe } \\
\text { [mo/yr, riser] }\end{array}$ \\
\hline \multirow{4}{*}{ AN-103 } & $3.74[5 / 96,1 \mathrm{~B}]$ & $2.92[9 / 96,12 \mathrm{~A}]$ & $3.64[8 / 96,15 \mathrm{~A}]$ \\
\hline & $3.86[5 / 96,16 \mathrm{~B}]$ & $3.86[2 / 00,11]$ & $3.71[3 / 99,15 \mathrm{~A}]$ \\
\hline & & & $3.71[5 / 00,15 \mathrm{~A}]$ \\
\hline & & & $3.71[7 / 00,15 \mathrm{~A}]$ \\
\hline \multirow{4}{*}{ AN-104 } & $3.72[3 / 96,16 \mathrm{~B}]$ & $4.4[9 / 96,10 \mathrm{~A}]$ & $4.47[8 / 96,15 \mathrm{~A}]$ \\
\hline & $4.12[4 / 96,1 \mathrm{~B}]$ & $3.65[8 / 96,12 \mathrm{~A}]$ & $4.17[4 / 99,15 \mathrm{~A}]$ \\
\hline & & $4.6[8 / 00,22]$ & $4.17[6 / 00,15 \mathrm{~A}]$ \\
\hline & & & $4.25[7 / 00,15 \mathrm{~A}]$ \\
\hline \multirow{5}{*}{ AN-105 } & $4.55[12 / 95,1 \mathrm{~B}]$ & $3.95[6 / 96,12 \mathrm{~A}]$ & $4.93[8 / 96,15 \mathrm{~A}]$ \\
\hline & $4.05[12 / 95,16 \mathrm{~B}]$ & $3.98[6 / 96,7 \mathrm{~B}]$ & $4.78[4 / 99,15 \mathrm{~A}]$ \\
\hline & & & $5.01[6 / 00,15 \mathrm{~A}]$ \\
\hline & & & $4.93[6 / 00,15 \mathrm{~A}]$ \\
\hline & & & $4.93[7 / 00,15 \mathrm{~A}]$ \\
\hline \multirow{5}{*}{ AW-101 } & $2.59[9 / 95,1 \mathrm{C}]$ & $2.9[5 / 96,24 \mathrm{~B}]$ & $2.57[6 / 97,15 \mathrm{~A}]$ \\
\hline & $2.78[9 / 95,13 \mathrm{~A}]$ & & $2.95[1 / 99,15 \mathrm{~A}]$ \\
\hline & & & $2.95[5 / 00,15 \mathrm{~A}]$ \\
\hline & & & $2.87[6 / 00,15 \mathrm{~A}]$ \\
\hline & & & $3.1[7 / 00,15 \mathrm{~A}]$ \\
\hline \multirow{10}{*}{ SY-103 } & $3.2[7 / 95,17 \mathrm{C}]$ & $3.37[9 / 94,14 \mathrm{~A}]$ & $2.57[4 / 96,17 \mathrm{~B}]$ \\
\hline & $3.29[8 / 95,22 \mathrm{~A}]$ & $2.9[3 / 00,17]$ & $3.556[4 / 97,17 \mathrm{~B}]$ \\
\hline & & $2.84[3 / 00,21]$ & $3.71[10 / 97,17 \mathrm{~B}]$ \\
\hline & & & $2.95[4 / 98,17 \mathrm{~B}]$ \\
\hline & & & $3.25[10 / 98,17 \mathrm{~B}]$ \\
\hline & & & $4.09[4 / 99,17 \mathrm{~B}]$ \\
\hline & & & $3.64[10 / 99,17 \mathrm{~B}]$ \\
\hline & & & $3.1[3 / 00,17 \mathrm{~B}]$ \\
\hline & & & $3.56[11 / 00,17 \mathrm{~B}]$ \\
\hline & & & $3.71[4 / 01,17 \mathrm{~B}]$ \\
\hline
\end{tabular}

The three data sources for sediment depth estimates are radial locations within the tank. To evaluate the potential for different sediment layer depths in hot and cold tank conditions, care must be taken to ensure that apparent depth variations with time are not just attributable to steady-state (with respect to bulk conditions, e.g. temperature) radial variations. Hot and cold tank data are available only at constant radial locations from the validation probes. 


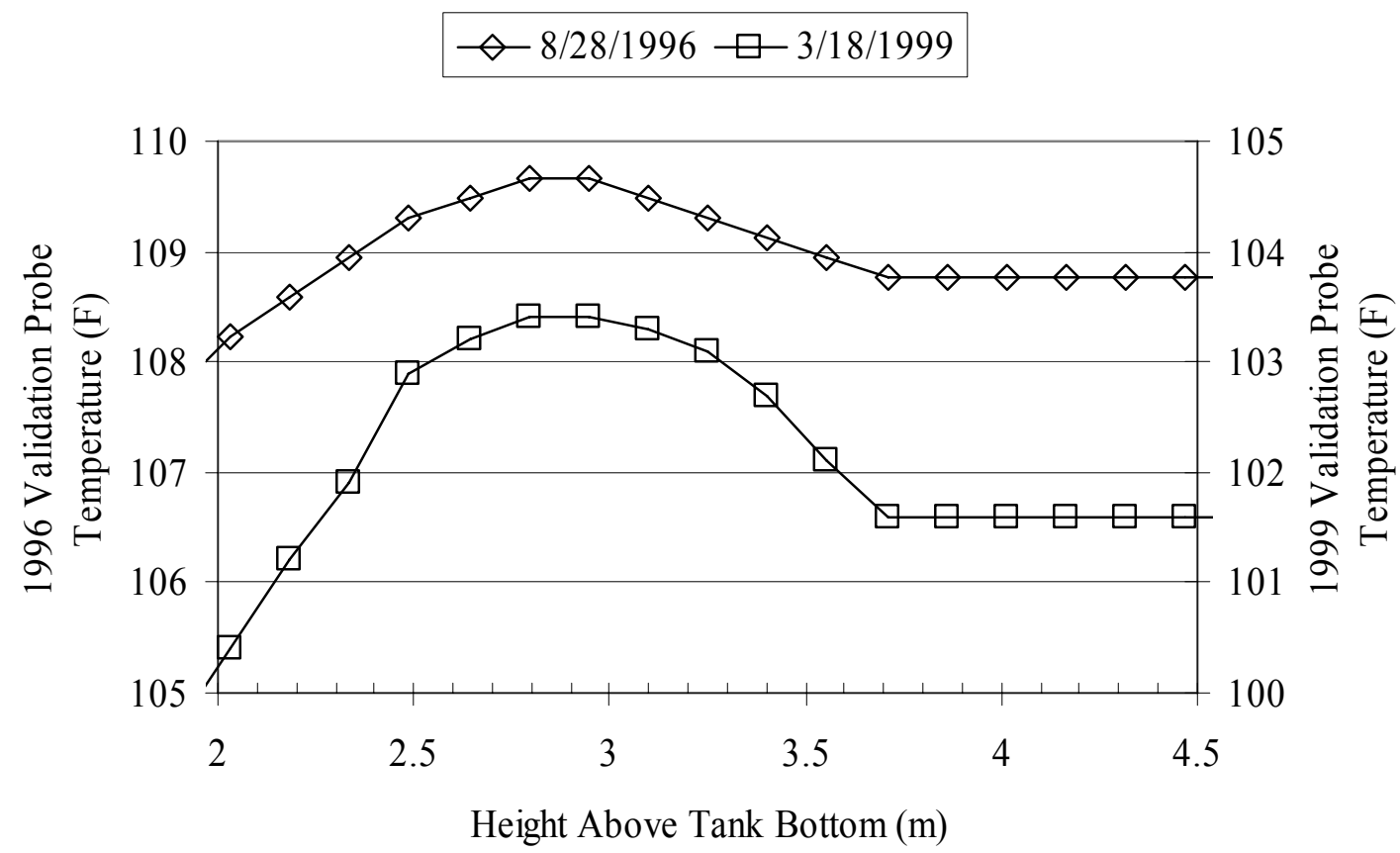

Figure 3.14. AN-103 Sediment-Liquid Interface Validation Probe Temperature Profile Examples

Aside from the level fluctuations apparent in SY-103, the only tank with an arguably significant change and trend in sediment depth over time from hot to cold conditions is AW-101 at approximately $0.53 \mathrm{~m}$ (Figure 3.15). The earliest validation probe data in AW-101 were taken after the hot-to-cold transition. The level fluctuations in SY-103 in the absence of significant temperature changes are not unexpected given the size and frequency of BDGREs in the tank (Hedengren et al. 2000).

Two additional arguments can be made in support of the observations from Figure 3.15 and Table 3.5. First, crust layer depth changes suggest solids precipitation from or transport to the layer were investigated. The crust layer thicknesses identified over time by the validation probe are presented in Figure 3.16. The thickness of the SY-103 crust fluctuates with time. The crusts in AN-104 and AN-105 appear to have slightly increased. AN-103's crust appears to have increased by approximately $0.31 \mathrm{~m}$, and the crust in AW-101 appears to have decreased by approximately $0.80 \mathrm{~m}$. Thus, the apparent sediment level increase in AW-101 may be attributable to solids settling from the crust layer. Hedengren et al. (2000) observed a thick (approaching $4 \mathrm{~m}$ ) convection-inhibiting layer from the base of the crust downward in AW-101. The 1996 validation probe data from AN-104 also showed this phenomenon as did most of the data from SY-103. Second, liquid density changes required to provide the necessary solids to increase the AW-101 sediment by approximately $0.53 \mathrm{~m}$ are considered. If the required density changes are very large, the apparent sediment depth change, neglecting potential crust effects, may not be plausible. 


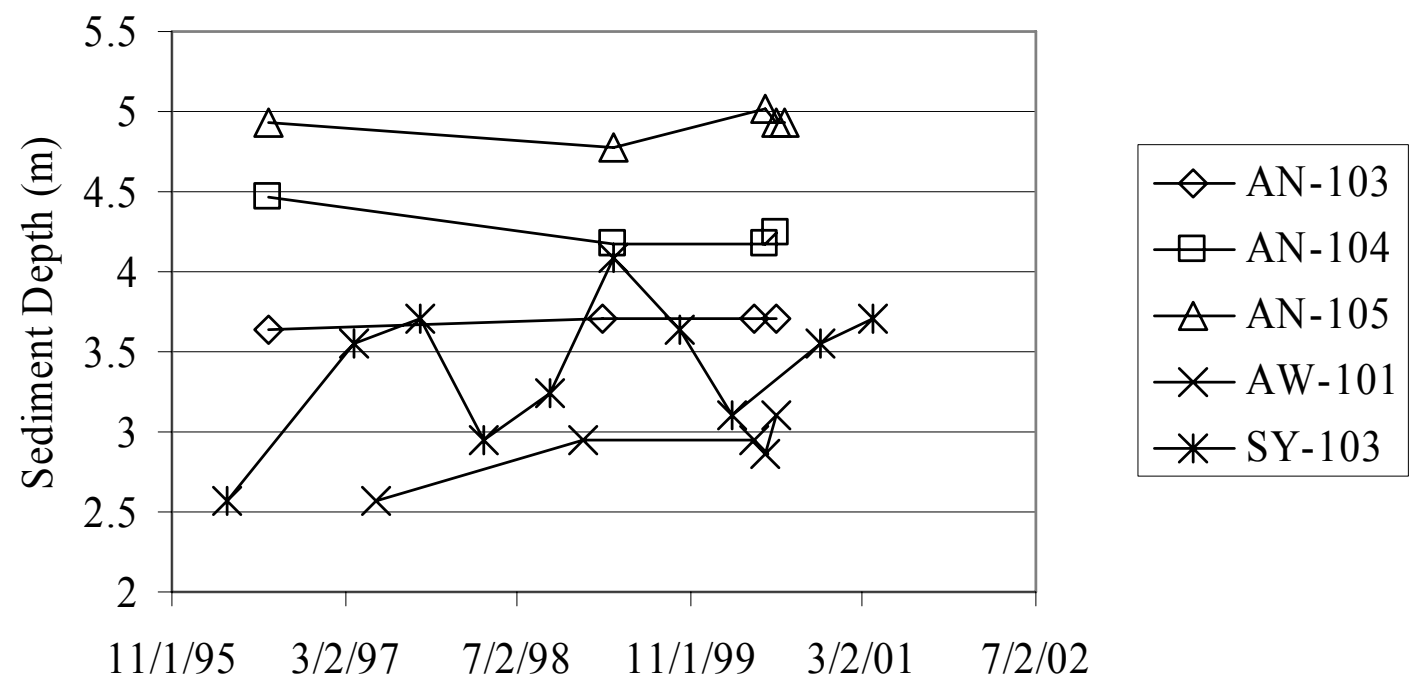

Validation Probe Date

Figure 3.15. Sediment Level Depth from Validation Probe Data as a Function of Time

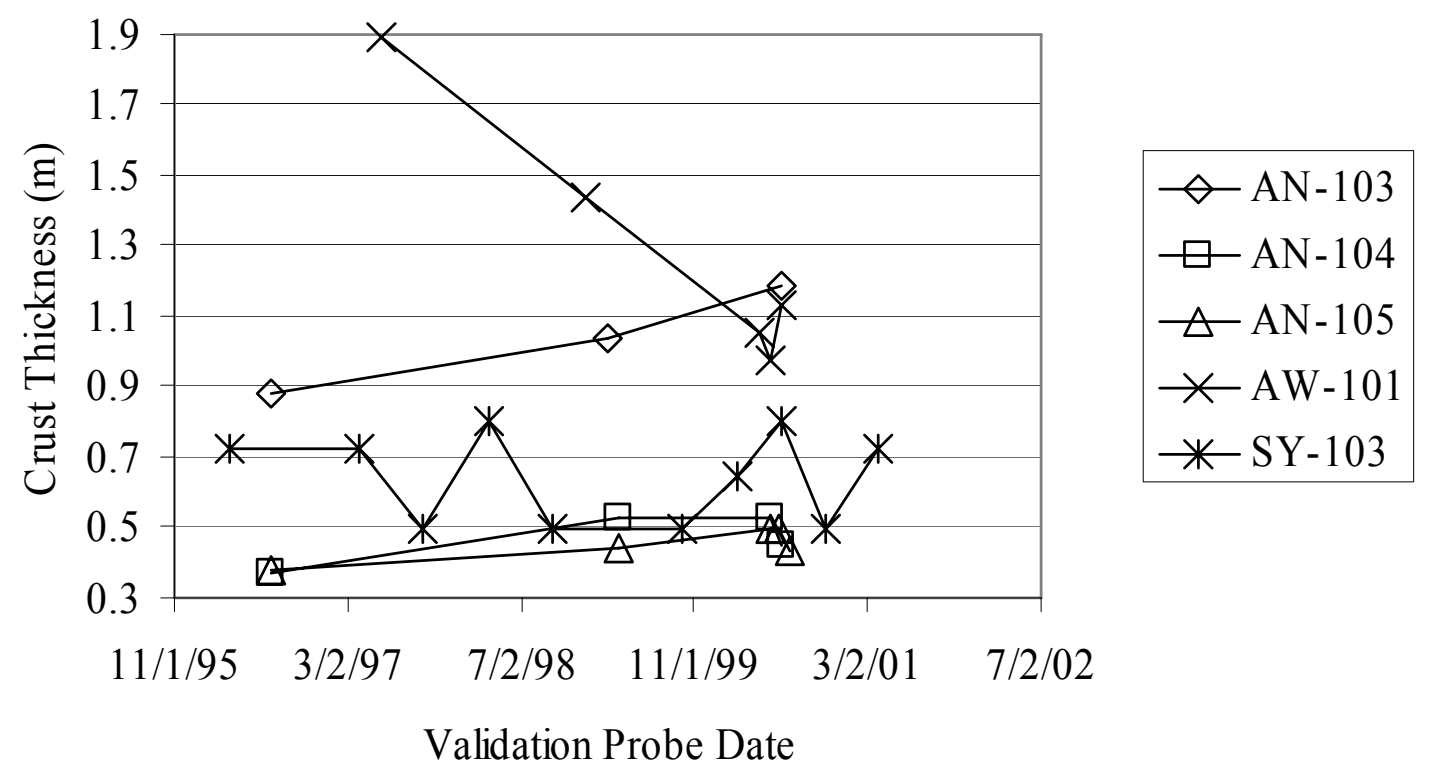

Figure 3.16. Crust Layer Thickness from Validation Probe Data as a Function of Time

It is assumed that the volume the solids occupy in their dissolved and undissolved states is constant. Thus, the required volume of solids to raise the sediment depth is equal to the volume of solids removed from the liquid. Considering only the liquid in the supernatant layer, the resulting decreased liquid density is 


$$
\rho_{\mathrm{L} 2}=\frac{\mathrm{H}_{\mathrm{L} 1} \rho_{\mathrm{L} 1}-\Delta \mathrm{H}_{\mathrm{S}} \phi_{2} \rho_{\mathrm{S}}}{\mathrm{H}_{\mathrm{L} 1}-\Delta \mathrm{H}_{\mathrm{S}} \rho_{\mathrm{S}}}
$$

where the subscripts 1 and 2 denote tank conditions before and after the sediment depth is increased by $\Delta H_{S}$. The liquid layer depth is denoted by $H_{L}$. Eq. (3.12) applied to AW-101 conditions with $\Delta \mathrm{H}_{\mathrm{S}}=0.53 \mathrm{~m}, \rho_{\mathrm{S}}=2.3 \mathrm{~g} / \mathrm{mL}, \mathrm{H}_{\mathrm{L} 1}=7.7 \mathrm{~m}$, and $\rho_{\mathrm{L} 1}=1.439 \mathrm{~g} / \mathrm{mL}$ results in $\rho_{\mathrm{L} 2}=$ $1.430 \mathrm{~g} / \mathrm{mL}$ with the solid volume fraction $\phi_{2}$ in $\Delta \mathrm{H}_{\mathrm{S}}$ set to that of the original sediment $(0.16$, Section 3.2.2). It is not expected that the solids volume fraction will be increased; decreasing $\phi_{2}$ results in a higher $\rho_{\mathrm{L} 2}$. The liquid density difference of $0.009 \mathrm{~g} / \mathrm{mL}$ is negligible. Thus, as supported by the crust thickness data, there is no plausible basis to argue against the apparent sediment level increase in AW-101. AW-101, therefore, is the only tank evaluated with different sediment depths for the hot and cold conditions.

As elucidated in Appendix D, sediment layer depth distributions were made based on combination of measurements, resulting in the weighted mean and median estimates presented in Table 3.6. The maximum and minimum values are the weighted median \pm 3 standard deviations. The standard deviations for hot and cold AW-101 are affected by the available data sources. This artifact is negated by assigning the average standard deviation of the other tanks. The difference in the median and mean in $\mathrm{AW}-101 \mathrm{C}$ is neglected given the altered standard deviation and assuming a normal distribution.

Table 3.6. Sediment Layer Depth Distributions

\begin{tabular}{||c|c|c|c|c|c|c||}
\hline Tank $^{(a)}$ & $\begin{array}{c}\text { Weighted } \\
\text { Median }\end{array}$ & $\begin{array}{c}\text { Weighted } \\
\text { Mean }\end{array}$ & Max & Min & $\begin{array}{c}\text { Standard } \\
\text { Deviation }\end{array}$ & Data Points \\
\hline AN-103 & 3.787 & 3.785 & 4.65 & 2.92 & 0.29 & 8 \\
\hline AN-104 & 3.954 & 3.957 & 4.89 & 3.03 & 0.31 & 9 \\
\hline AN-105 & 4.360 & 4.358 & 4.82 & 3.90 & 0.154 & 9 \\
\hline AW-101H & 2.687 & 2.687 & 3.55 & 1.83 & 0.287 & 3 \\
\hline AW-101C & 2.950 & 2.888 & 3.75 & 2.03 & 0.287 & 5 \\
\hline SY-103 & 3.273 & 3.260 & 4.44 & 2.08 & 0.395 & 15 \\
\hline (a) "H" or "C" with tank name indicates "hot"or "cold" tank state. \\
\hline
\end{tabular}

The standard deviations are such that application in a normal distribution results in sediment depths that are expected to bound the measurements (compare Tables 3.4 and 3.5). The exception is the maximum $\mathrm{AN}-105$ depth, a result of the weighting methodology that takes into account the relative accuracy of the measurements (see Appendix D). 


\subsubsection{Gas Generation Rate}

The volumetric gas generation rate in moles $/ \mathrm{m}^{3} /$ day in the sediment layer is determined from the hydrogen generation rate by

$$
\mathrm{G}=\frac{\mathrm{G}_{\mathrm{H}}}{\left[\mathrm{H}_{2}\right]_{\mathrm{gas}}}
$$

where $\mathrm{G}_{\mathrm{H}}$ is the volumetric hydrogen generation rate, and $\left[\mathrm{H}_{2}\right]_{\mathrm{gas}}$ is the hydrogen fraction of the retained gas. Tank-specific values for the hydrogen fraction in the retained gas are presented in Section 3.2.6.

Hydrogen generation rates in the Hanford waste tanks are reported in $\mathrm{Hu}$ (2004). Given that the tank conditions for $\mathrm{Hu}$ (2004) were altered from the historic states considered in this evaluation, the rates were recomputed with Hu's model.(a) Required inputs include specific chemical analytes, sediment and liquid densities, liquid fraction in the sediment, waste temperature, and sediment depth. The sediment and liquid densities, waste temperatures, and sediment depths are taken from Sections 3.2.1, 3.2.2, and 3.2.4. The remaining inputs were taken from the Best Basis Inventory (BBI).(b) Generation rates were computed for both the hot and cold tanks. The cold tank gas generation rates range from 54\% (AN-104) to 68\% (AW-101) of the hot tank results.

Combining BBI data with the above-determined layer density values (Section 3.2.2) may cause inconsistencies in the liquid fraction in the sediment. As discussed in Section 3.2.2, determining solid/liquid content in a solid-liquid waste matrix with water content data from the bulk solid and liquid may produce results that are not in agreement with the waste chemistry. However, this potential effect is not thought to be important in the current analysis given the uncertainties associated with the hydrogen generation rate, specifically of the sediment, as well as the overall uncertainty of the model itself. Further, the $\mathrm{Hu}$ (2004) model was calibrated to waste data using water content data to determine the sediment liquid fraction, so application of a different methodology may be inconsistent.

The uncertainty of the calculated hydrogen generation rate is determined using the methodology presented in Barker and Hedengren (2004). A skewed-normal distribution of $1.1 \mathrm{G}_{\mathrm{H}}$ to $\mathrm{G}_{\mathrm{H}} / 2$ is used for each tank. This distribution varies on the lower end from that given in Barker and Hedengren (2004) because the model and field-estimated hydrogen generation rates are only considered for the specific tanks of concern.

(a) Personal communication from TA Hu, CH2M HILL, Richland, Washington.

(b) Best Basis Inventory, http://twins.pnl.gov/twins3/twins.htm. Personal communication from TA Hu, CH2M HILL, Richland, Washington. 
Estimates for the total gas generation rate in SY-101 during the time of BDGRE behavior vary from $140 \mathrm{ft}^{3} /$ day (Babad et al. 1991) to $110 \mathrm{ft}^{3} /$ day (Alleman et al. 1994). Gas generation estimates from after mixer pump installation and operation are approximately $100 \mathrm{ft}^{3} /$ day. (a) Assuming a uniform volumetric gas generation rate for the supernatant and sediment layers (although the sediment, which is approximately $50 \%$ of the waste by volume, has less liquid content, it has an elevated temperature, so the generation rate is assumed volumetrically uniform) and a hydrogen concentration of 0.29 (see Section 2.2.6), the volumetric hydrogen generation rate is taken to be 0.0113 to $0.0081 \mathrm{moles} / \mathrm{m}^{3} /$ day. A normal distribution is assumed, with a median of $0.0097 \mathrm{moles} / \mathrm{m}^{3} /$ day. The calculated volumetric hydrogen generation rates in the sediment layer are included in the data summary in Section 3.2.8.

\subsubsection{Retained Gas Composition}

As specified in Section 3.2.5, the total volumetric gas generation rate in the sediment is determined from the hydrogen generation rate and concentration in the retained gas. Direct in situ measurements for retained gas composition in the sediment of AN-103, AN-104, AN-105, AW-101, and SY-101 are available from Mahoney et al. (1999). Sullivan (1995) provides a best estimate of the hydrogen concentration in SY-101 (0.29, ranging from 0.29 to 0.31 ). This result is corroborated by Alleman et al. (1994) and Mahoney et al. (1999) and is applied in this analysis as most representative of tank conditions during SY-101's BDGRE period.

No measurements are available for the retained gas composition in SY-103. The hydrogen fraction in AN-103, AN-104, AN-105, AW-101, and SY-101 is normally distributed. Therefore, the hydrogen fraction in SY-103 was assumed to be normal with maximum and minimum values taken from the combination of the other tanks. The hydrogen concentrations in the retained gas in the sediment layer are included in the data summary in Section 3.2.8.

\subsubsection{Peak GRE Hydrogen Concentrations}

Gas release events in tanks AN-103, AN-104, AN-105, AW-101, SY-103, and SY-101 were recorded by headspace gas monitoring equipment through September 2002 (Hedengren 2003). Headspace gas concentrations after this date are not evaluated due to stand-by status, disconnection, or lack of maintenance of the gas monitoring equipment. (b) A detailed analysis of these data for AN-103, AN-104, AN-105, and AW-101 through June 30, 2001 was conducted in Wells et al. (2002), where it was identified that, similar to the historic BDGREs in SY-101, many of the events were multiple BDGREs, though only one was usually significant. Wells et al.

(a) Antoniak ZI, ME Brewster, EJ Eschbach, JG Fadeff, JR Friley, NB Gallagher, JD Hudson, JJ Irwin, JA Lechelt, FE Panisko, RM Riensche, CW Stewart, and NE Wilkins. 1995. Preliminary Quarterly Review of 241-SY-101 Mixer Pump Data. Pacific Northwest Laboratory, Richland, Washington.

(b) Personal communication from DC Hedengren, CH2M HILL, Richland, Washington. 
(2002) determined the number of BDGREs per event, time to peak gas release rate, and total gas release volume for each event. Similar analyses have been conducted for SY-103.

The hazard from a GRE is determined by the peak hydrogen concentration in the headspace. As presented in Section 3.3.1, the gas release volumes are computed from the peak headspace hydrogen concentration as a function of the headspace and hydrogen fraction in the released gas. (a) The peak hydrogen concentrations for GREs are listed in Table 3.7.

The data from the analyses in Wells et al. (2002) are supplemented by Hedengren (2003). Hedengren et al. (2000) note that, in general, the GRE volumes appear to decrease over time. Further, some tanks appear to experience a decreasing GRE frequency over the years, while others show an increase. There is a distinct scarcity of GREs in 1996 and 1997, with activity picking up again in 1998. Some tanks had no events at all in 1996 or 1997 but multiple events in 1995 and 1998. There is considerable variation in the release volume and frequency from tank to tank. SY-103 is the exception and appears to maintain consistent GRE behavior. The observed distinctions (AN-103, AN-104, AN-105, and AW-101) and similarities (SY-103) are consistent with the hot and cold tank periods (Section 3.2.1). The GREs identified from headspace gas concentration data for different temperature periods are denoted as such in Table 3.7. In subsection 3.2.7.1, distributions representing the tank's GRE history are presented, and the largest reasonably expected BDGRE is identified.

In addition to the headspace gas concentration data, GREs may, in theory, be identified by sudden waste surface level drops as gas is released from the waste. In practice, however, irregularities in the waste surface (the historic BDGRE tanks all have floating crust layers), level instrument maintenance and different instrumentation make the waste surface level history an imprecise indication of gas release. Further, there has historically been no correlation between level drop and GREs in AN-103, AN-104, and AW-101 (Hedengren et al. 2000). The level drop method for identifying GREs has therefore been discarded except in the case of very large GREs. Though not able to provide quantifiable results, the absence of gas concentration data since September 2002 suggests that the waste surface level history should at least be considered. This consideration is made in subsection 3.2.7.2.

(a) The gas release volume is computed directly from the peak hydrogen concentration and thus represents the gas release volume necessary to reach the indicated hydrogen concentration. Gas release volumes computed in this fashion will thus underestimate the total gas release volume for a BDGRE. 
Table 3.7. GRE Peak Hydrogen Concentrations

\begin{tabular}{|c|c|c|c|c|c|c|c|c|c|c|c|}
\hline Tank & GRE Date & $\begin{array}{c}\operatorname{Max~H}_{2} \\
(\mathrm{ppm})\end{array}$ & Tank & GRE Date & $\begin{array}{c}\mathrm{Max} \mathrm{H}_{2} \\
\text { (ppm) }\end{array}$ & Tank & GRE Date & $\begin{array}{c}\operatorname{Max~H}_{2} \\
\text { (ppm) }\end{array}$ & Tank & GRE Date & $\begin{array}{c}\operatorname{Max} \mathrm{H}_{2} \\
\text { (ppm) }\end{array}$ \\
\hline $\mathrm{AN}-103 \mathrm{H}$ & $8 / 22 / 95$ & 1,600 & \multirow[t]{25}{*}{$\mathrm{AN}-105 \mathrm{C}$} & $9 / 26 / 97$ & 770 & \multirow[t]{22}{*}{ AW-101H } & $10 / 2 / 94$ & 5,500 & \multirow[t]{37}{*}{ SY-103 } & $1 / 22 / 95$ & 1,090 \\
\hline \multirow[t]{9}{*}{$\mathrm{AN}-103 \mathrm{C}$} & $4 / 11 / 98$ & 960 & & $11 / 22 / 97$ & 1,030 & & $10 / 4 / 94$ & 6,200 & & $3 / 2 / 95$ & 2,210 \\
\hline & $2 / 1 / 99$ & 540 & & $12 / 31 / 97$ & 3,940 & & $10 / 21 / 94$ & 2,980 & & $5 / 3 / 95$ & 1,430 \\
\hline & $9 / 4 / 99$ & 1,080 & & $9 / 2 / 98$ & 2,230 & & $11 / 28 / 94$ & 4,900 & & $8 / 24 / 95$ & 1,260 \\
\hline & $12 / 30 / 99$ & 580 & & $10 / 26 / 98$ & 5,040 & & $2 / 23 / 95$ & 4,600 & & 9/7/95 & 1,890 \\
\hline & $8 / 6 / 00$ & 210 & & $3 / 7 / 99$ & 760 & & $5 / 8 / 95$ & 1,800 & & $12 / 3 / 95$ & 206 \\
\hline & $10 / 3 / 00$ & 260 & & $5 / 2 / 99$ & 182 & & $5 / 17 / 95$ & 1,000 & & $6 / 7 / 96$ & 1,090 \\
\hline & $10 / 20 / 00$ & 340 & & $7 / 29 / 99$ & 6,208 & & $7 / 8 / 95$ & 2,000 & & $7 / 14 / 96$ & 2,110 \\
\hline & $8 / 13 / 01$ & 760 & & $8 / 2 / 99$ & 3,839 & & $7 / 12 / 95$ & 900 & & $12 / 20 / 96$ & 5,110 \\
\hline & $10 / 20 / 01$ & 480 & & $10 / 27 / 99$ & 180 & & $8 / 2 / 95$ & 3,300 & & $11 / 28 / 97$ & 1,990 \\
\hline & $2 / 6 / 02$ & 230 & & $12 / 31 / 99$ & 186 & & $9 / 14 / 95$ & 1,930 & & $12 / 7 / 97$ & 2,060 \\
\hline & $11 / 6 / 94$ & 3,050 & & $7 / 21 / 00$ & 146 & & $9 / 23 / 95$ & 1,460 & & $8 / 15 / 98$ & 1,620 \\
\hline \multirow{7}{*}{ AN-104H } & $2 / 16 / 95$ & 2,089 & & $10 / 28 / 00$ & 204 & & $9 / 24 / 95$ & 4,660 & & $10 / 23 / 98$ & 100 \\
\hline & $8 / 4 / 99$ & 480 & & $11 / 27 / 00$ & 151 & & $10 / 16 / 95$ & 1,750 & & $12 / 25 / 98$ & 2,854 \\
\hline & $10 / 2 / 95$ & 3,068 & & $12 / 14 / 00$ & 164 & & $12 / 12 / 95$ & 2,110 & & $1 / 10 / 99$ & 580 \\
\hline & $10 / 5 / 95$ & 1,000 & & $2 / 23 / 01$ & 82 & & $12 / 29 / 95$ & 6,000 & & $3 / 9 / 99$ & 570 \\
\hline & $10 / 8 / 95$ & 1,800 & & $3 / 21 / 01$ & 869 & & $1 / 13 / 96$ & 1,170 & & $5 / 30 / 99$ & 950 \\
\hline & $5 / 4 / 96$ & 6,109 & & $3 / 22 / 01$ & 589 & & $1 / 25 / 96$ & 1,830 & & $10 / 25 / 99$ & 1,130 \\
\hline & $5 / 2 / 97$ & 2,250 & & $3 / 24 / 01$ & 166 & & $2 / 5 / 96$ & 3,200 & & $10 / 26 / 99$ & 1,020 \\
\hline \multirow[t]{15}{*}{ AN-104C } & $8 / 25 / 98$ & 310 & & $5 / 26 / 01$ & 120 & & $2 / 14 / 96$ & 940 & & $11 / 26 / 99$ & 450 \\
\hline & $9 / 26 / 98$ & 310 & & $6 / 24 / 01$ & 5,735 & & $5 / 14 / 96$ & 1,381 & & $12 / 3 / 99$ & 570 \\
\hline & $12 / 2 / 98$ & 550 & & $10 / 22 / 01$ & 329 & & $6 / 4 / 96$ & 2,519 & & $1 / 16 / 00$ & 1,100 \\
\hline & $4 / 26 / 99$ & 135 & & $11 / 22 / 01$ & 213 & \multirow[t]{15}{*}{ AW-101C } & $4 / 21 / 98$ & 398 & & $2 / 12 / 00$ & 390 \\
\hline & $6 / 1 / 99$ & 151 & & $12 / 13 / 01$ & 191 & & $5 / 1 / 98$ & 530 & & $3 / 13 / 00$ & 1,090 \\
\hline & $7 / 2 / 99$ & 241 & & $2 / 7 / 02$ & 144 & & $6 / 24 / 98$ & 450 & & $9 / 22 / 00$ & 1,290 \\
\hline & $8 / 1 / 99$ & 181 & SY-101 & $4 / 19 / 90$ & 35,000 & & $7 / 4 / 98$ & 480 & & $10 / 28 / 00$ & 1,070 \\
\hline & $9 / 4 / 99$ & 152 & & $8 / 5 / 90$ & 12,000 & & $1 / 16 / 00$ & 205 & & $11 / 2 / 00$ & 880 \\
\hline & $5 / 3 / 00$ & 265 & & $10 / 24 / 90$ & 47,000 & & $4 / 22 / 00$ & 2,094 & & $10 / 23 / 01$ & 1,900 \\
\hline & $6 / 2 / 00$ & 264 & & $2 / 16 / 91$ & 400 & & $9 / 17 / 00$ & 225 & & $11 / 7 / 01$ & 540 \\
\hline & $11 / 4 / 00$ & 122 & & $5 / 16 / 91$ & 28,000 & & $9 / 19 / 00$ & 212 & & $11 / 18 / 01$ & 500 \\
\hline & $11 / 6 / 00$ & 370 & & $8 / 27 / 91$ & 3,800 & & $9 / 22 / 00$ & 704 & & $11 / 27 / 01$ & 1,000 \\
\hline & $1 / 3 / 01$ & 119 & & $12 / 4 / 91$ & 53,000 & & $10 / 30 / 00$ & 259 & & $1 / 10 / 02$ & 580 \\
\hline & $2 / 28 / 01$ & 351 & & $4 / 20 / 92$ & 14,800 & & $11 / 19 / 00$ & 567 & & $2 / 4 / 02$ & 370 \\
\hline & $2 / 19 / 02$ & 128 & & $9 / 3 / 92$ & 51,200 & & $12 / 10 / 00$ & 1,162 & & $2 / 9 / 02$ & 860 \\
\hline \multirow[t]{2}{*}{$\mathrm{AN}-105 \mathrm{H}$} & $8 / 21 / 95$ & 17,000 & & $2 / 2 / 93$ & 27,400 & & $11 / 13 / 01$ & 200 & & $2 / 11 / 02$ & 700 \\
\hline & $5 / 30 / 96$ & 14,500 & & $6 / 26 / 93$ & 34,000 & & $11 / 26 / 01$ & 160 & & $2 / 21 / 02$ & 380 \\
\hline & $4 / 6 / 97$ & 6,980 & & & & & $12 / 3 / 01$ & 490 & & $2 / 25 / 02$ & 660 \\
\hline
\end{tabular}




\subsubsection{Peak Hydrogen Concentration Distributions}

The current analysis considers the flammability hazard of BDGREs. Thus, the most probable peak hydrogen concentration, or, more conservatively, that which represents a specific confidence bound, must be identified. The $95^{\text {th }}$ percentile value is selected as the largest reasonably expected BDGRE and is identified by determining a distribution for each data set.

The raw data from Table 3.7 were used to generate histograms to identify plausible distribution types. With this basis, the distributions specified in Table 3.8 were developed to represent the data. These distributions are presented as cumulative probabilities with the BDGRE data for each tank in Figures 3.17 through 3.25. AN-103H, with its single identified BDGRE, is not analyzed.

Table 3.8. Peak Headspace Hydrogen Concentration Distributions (ppm)

\begin{tabular}{||l|c|c|c|l||}
\hline \multicolumn{1}{|c|}{ Tank $^{(\text {a) }}$} & Median & Mean & 95th Percentile & \multicolumn{1}{c|}{ Distribution } \\
\hline AN-103C & 510 & 554 & 1,040 & Manipulated Beta \\
\hline AN-104H & 2,169 & 2,412 & 5,013 & Manipulated Beta \\
\hline AN-104C & 241 & 262 & 486 & Manipulated Beta \\
\hline AN-105H & 14,431 & 14,505 & 23,054 & Truncated Normal \\
\hline AN-105C & 1,338 & 1,591 & 3,915 & Manipulated Beta \\
\hline AW-101H & 2,054 & 2,249 & 4,194 & Truncated Normal \\
\hline AW-101C & 502 & 560 & 1,180 & Truncated Normal \\
\hline SY-101 & 27,950 & 28,002 & 52,999 & Truncated Normal \\
\hline SY-103 & 1,020 & 1,141 & 2,564 & Uniform \\
\hline (a) "H" or "C" with tank name indicates "hot" or "cold" tank state. \\
\hline \multicolumn{5}{|l}{} \\
\hline
\end{tabular}

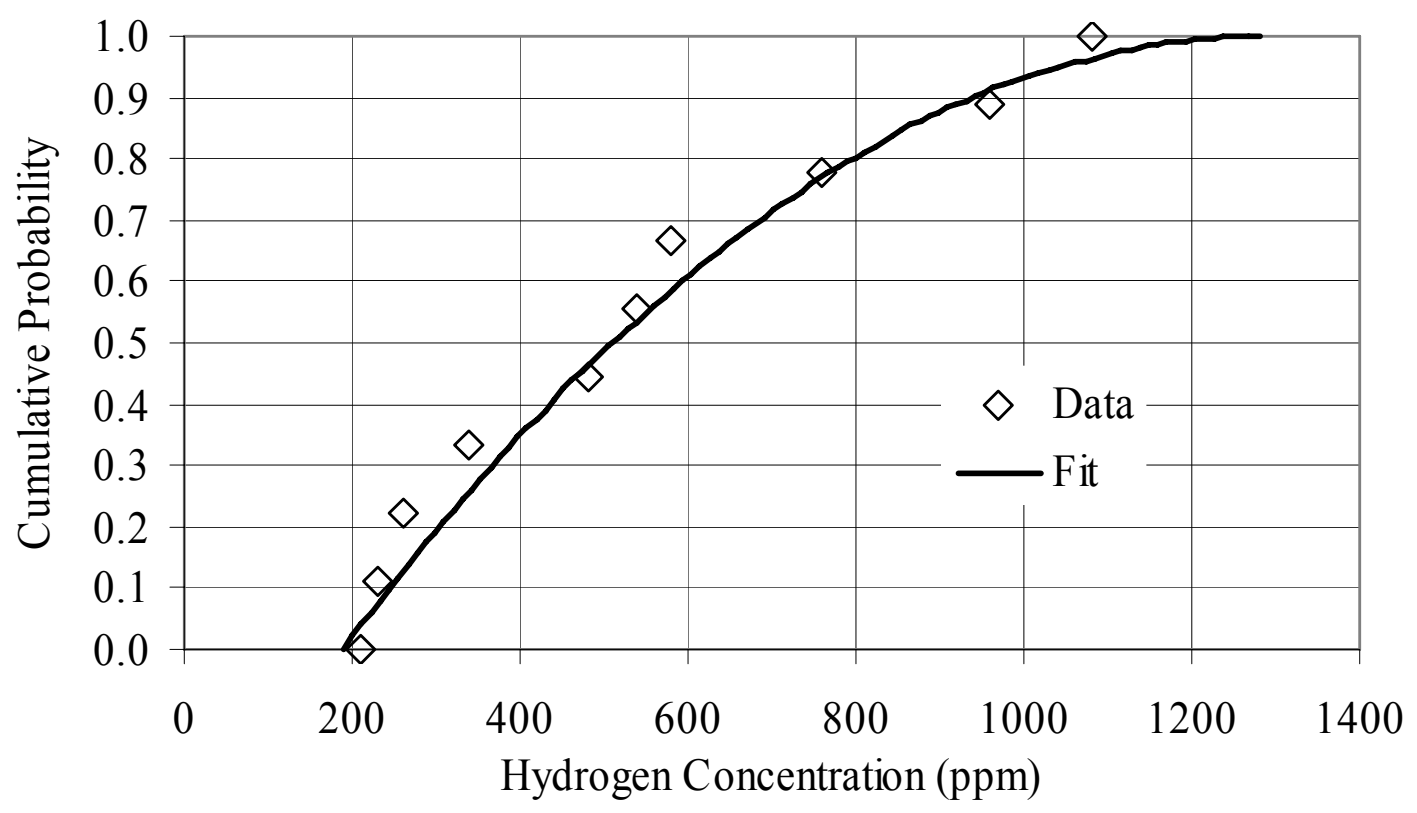

Figure 3.17. AN-103C BDGRE Data and Distribution 


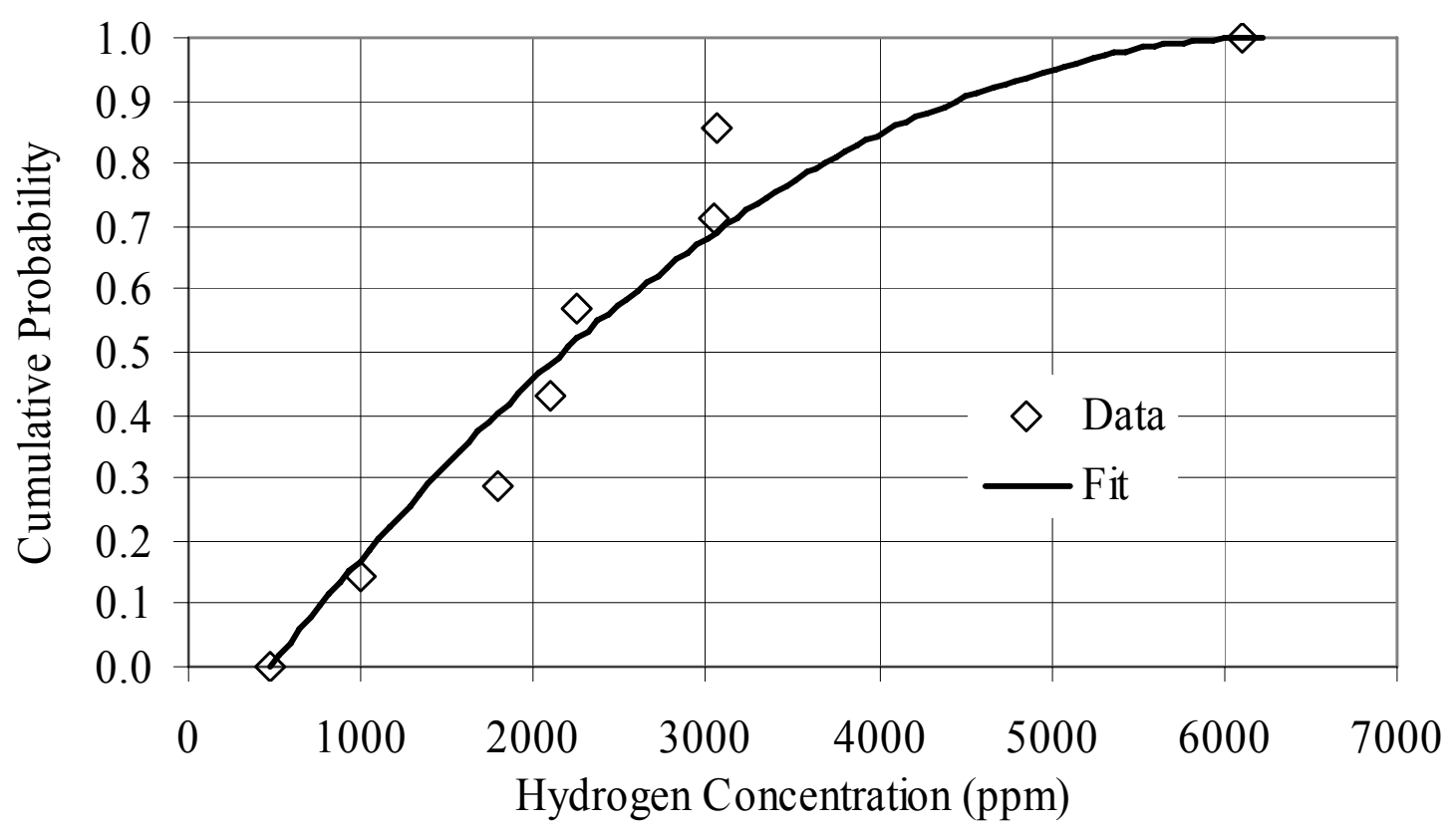

Figure 3.18. AN-104H BDGRE Data and Distribution

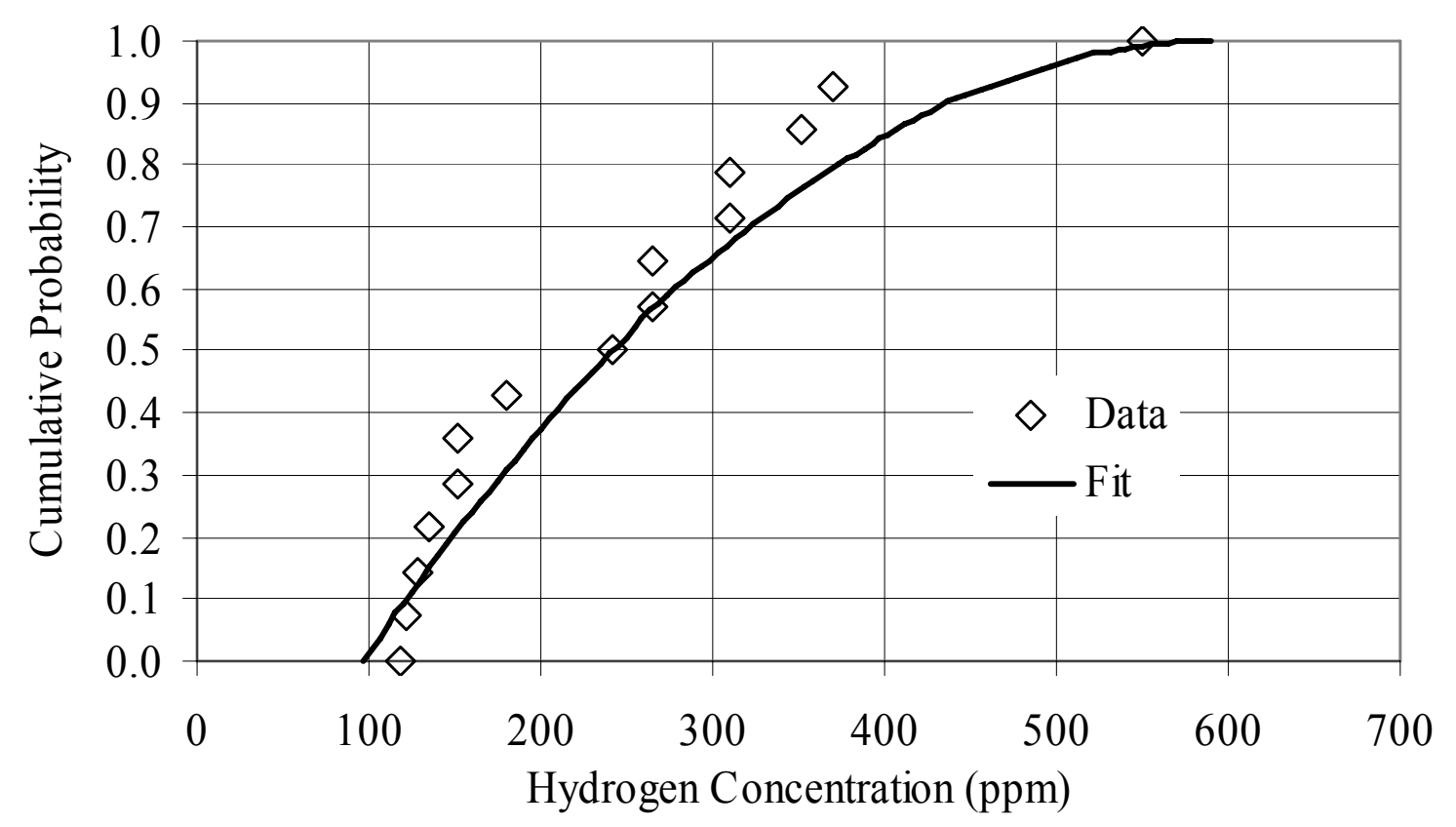

Figure 3.19. AN-104C BDGRE Data and Distribution 


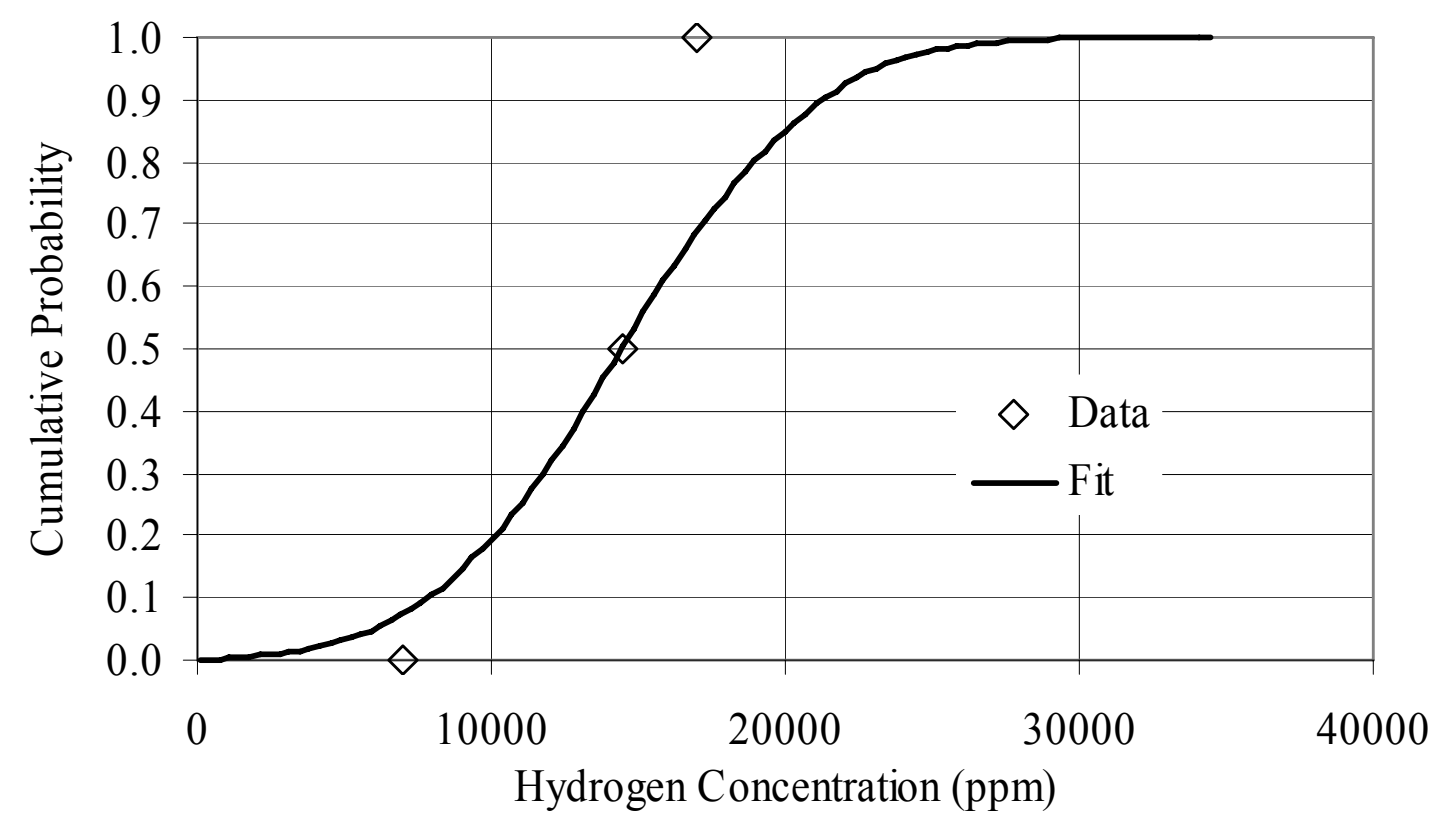

Figure 3.20. AN-105H BDGRE Data and Distribution

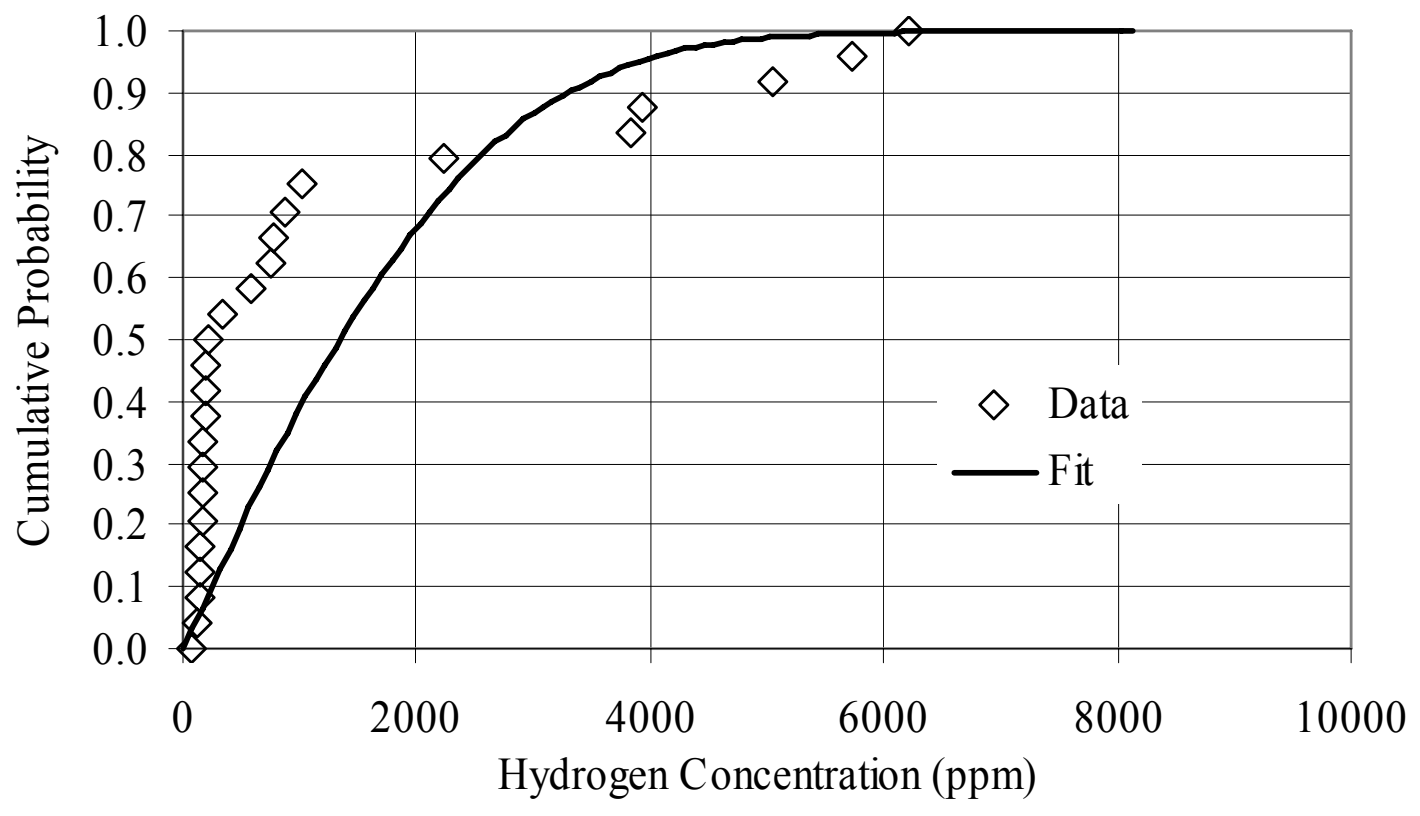

Figure 3.21. AN-105C BDGRE Data and Distribution 


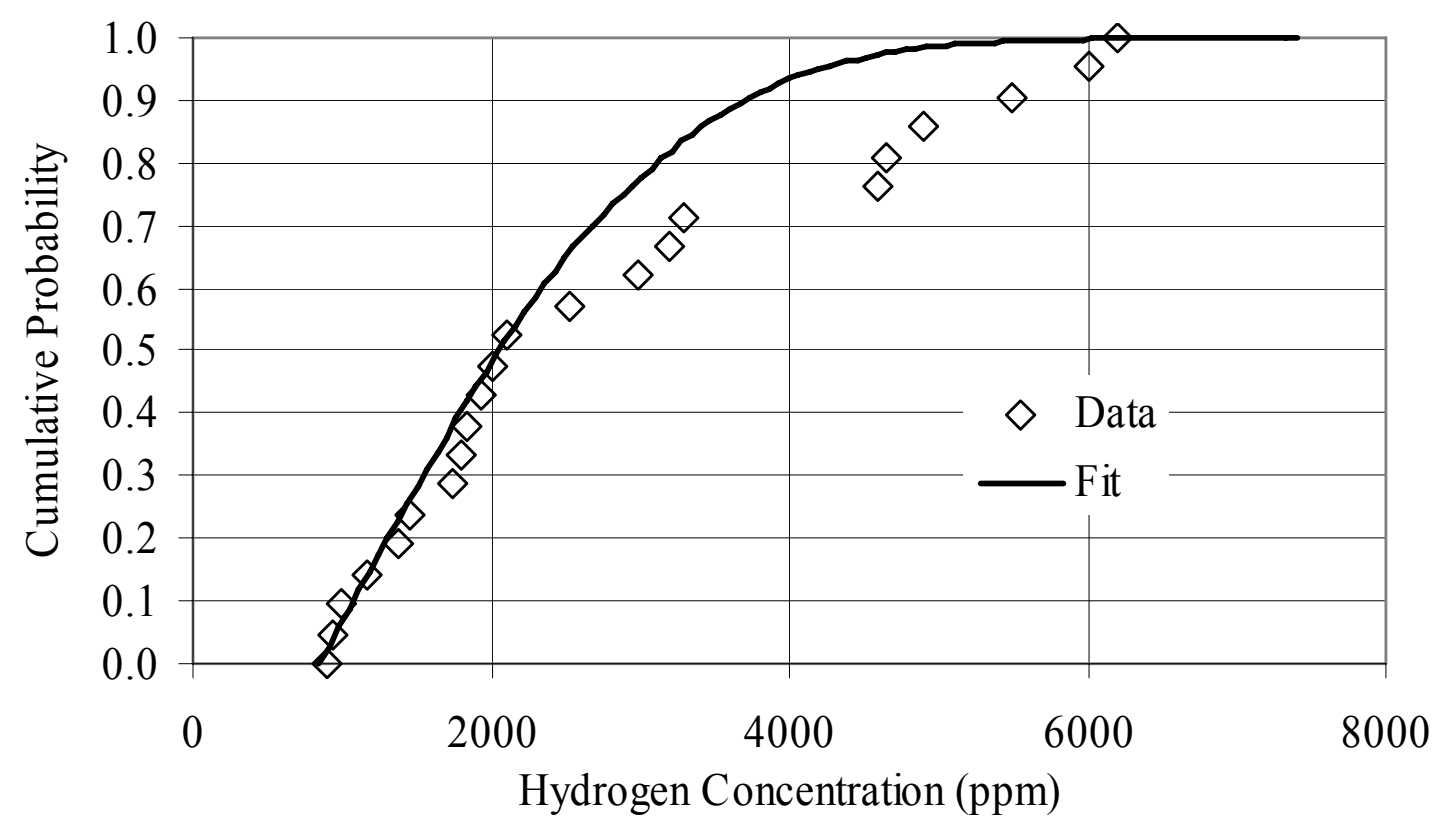

Figure 3.22. AW-101H BDGRE Data and Distribution

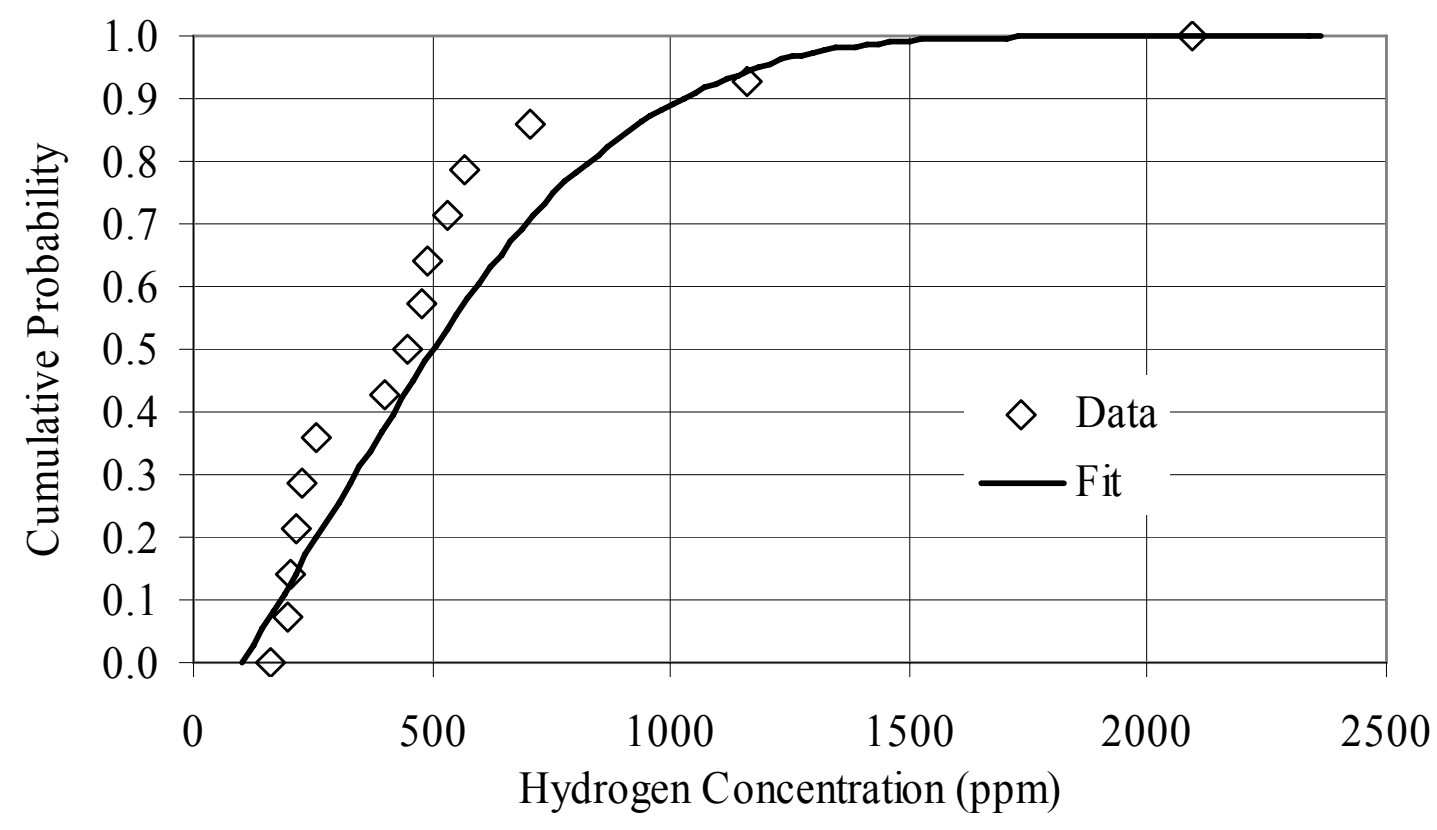

Figure 3.23. AW-101C BDGRE Data and Distribution 


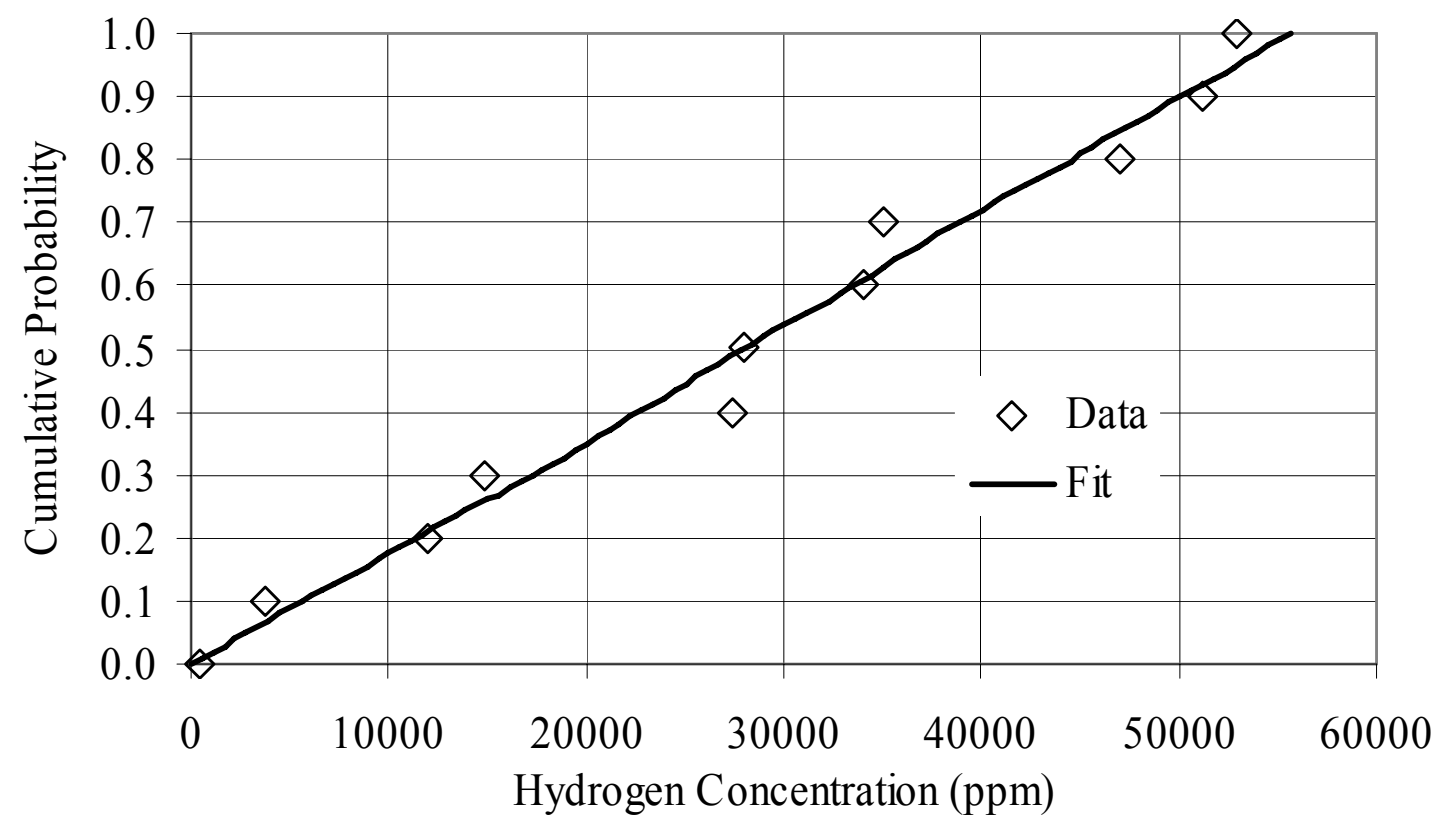

Figure 3.24. SY-101 BDGRE Data and Distribution

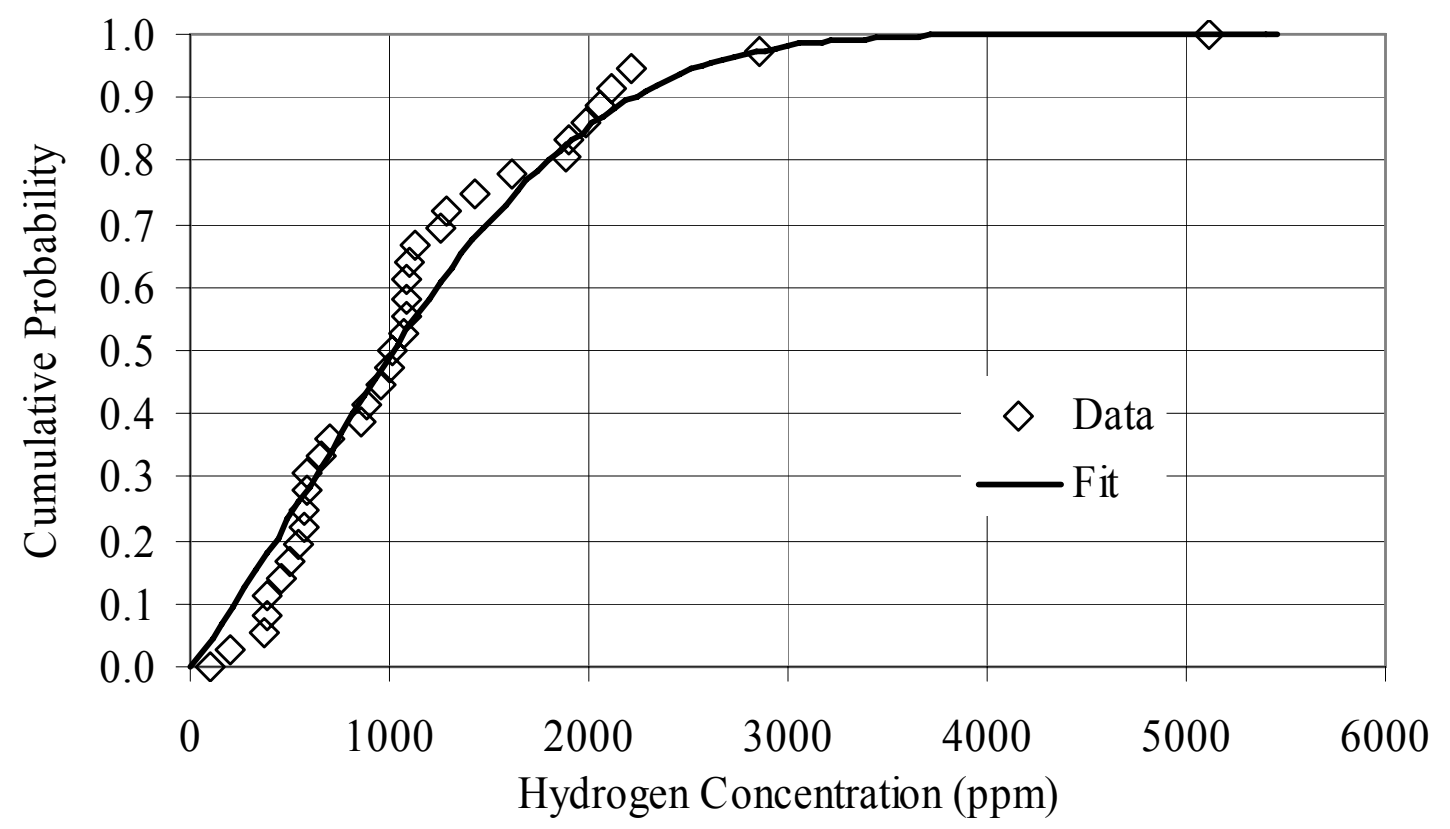

Figure 3.25. SY-103 BDGRE Data and Distribution 
For AN-105C, AW-101H, and AW-101C, the $95^{\text {th }}$ percentile of the data is apparently greater than that of the distribution fits (Figures 3.26 and 3.27). This is due to using a distribution that best fits the data as a whole rather than a combination of distributions to match the specific quantiles. This approach was used given the relatively sparse data sets. Further, the specific tanks in question have minimal effect on the final results.

\subsubsection{Surface Level History Observations}

The surface level histories to April 25, 2005 were qualitatively investigated for BDGRE behavior, as shown in the following figures. For each tank, the initial plot is similar to that of Hedengren et al. (2000). GRE histories from Table 3.8 are shown as the vertical lines. The surface level history for AN-103 is shown in Figures 3.26 and 3.27. It can be argued, as is physically plausible and observed specifically in SY-103, that BDGREs have historically occurred in AN-103 only after a period of level growth, or gas retention (Figure 3.26). This cyclic behavior continued in 2000 and 2001, and the level history suggests possible BDGREs in 2002 (Figure 3.27). The surface level since 2002 has not continued its historical cyclic behavior, possibly indicating a behavioral change in gas retention and release characteristics. A level drop is noted from May 26 to May 27, 2003, possibly indicating a BDGRE, though there is no historical correlation of level drops and BDGREs in AN-103.

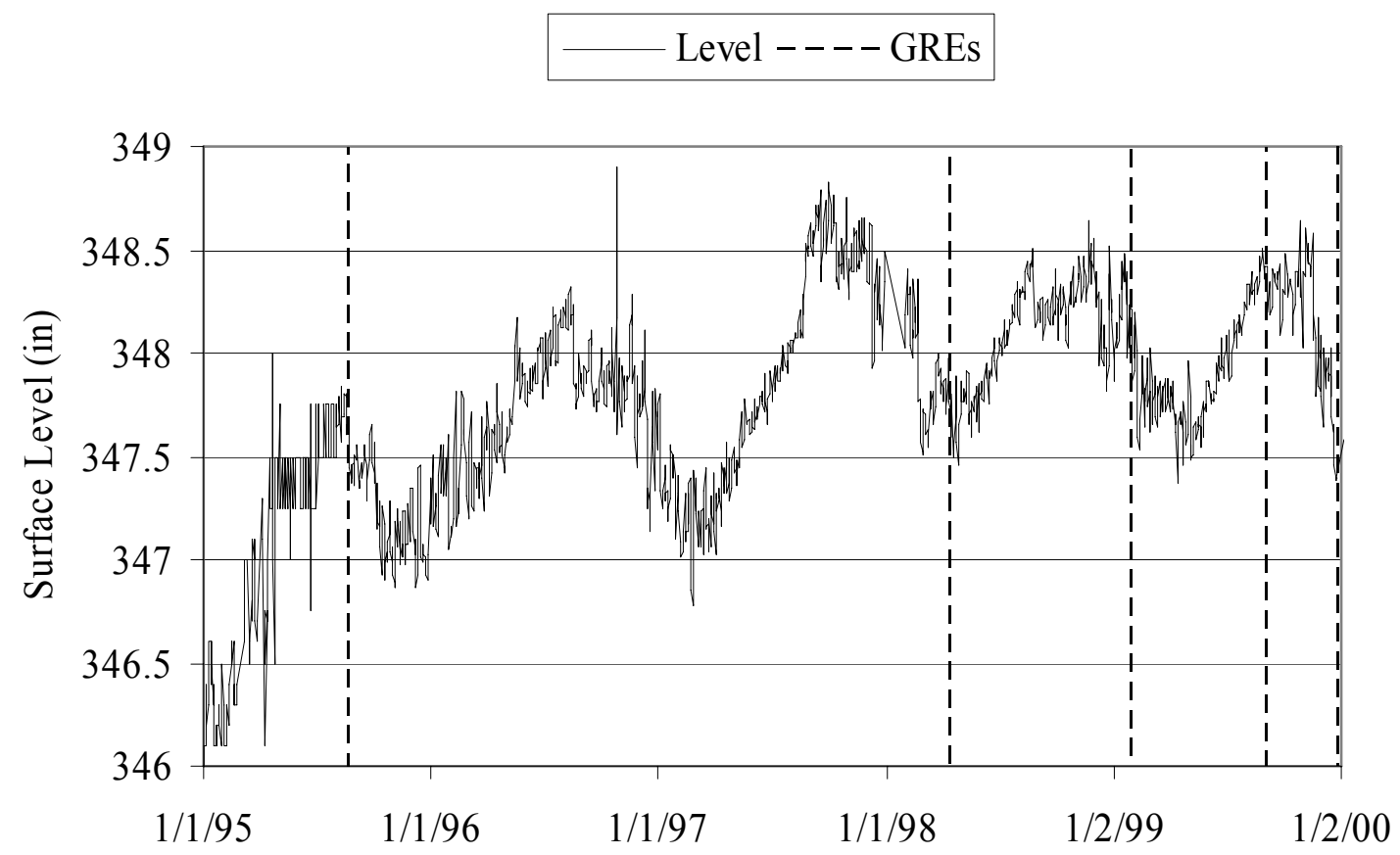

Figure 3.26. AN-103 Surface Level and GRE History, 1/95-1/00 


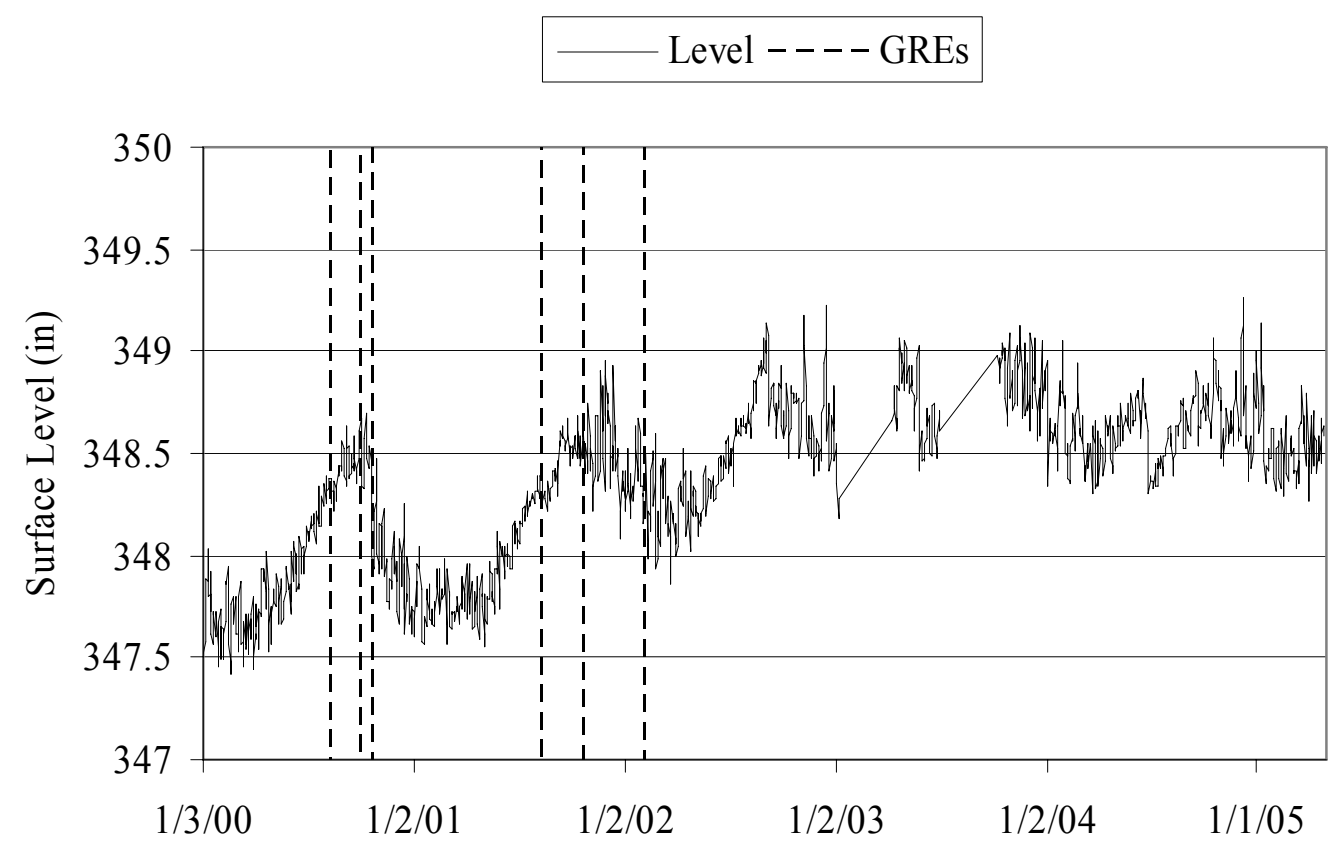

Figure 3.27. AN-103 Surface Level and GRE History, 1/00-4/05

The level trend change observed in AN-104 is a cessation of the slow decrease (Figures 3.28 and 3.29). Though several abrupt level drops appear that were not associated with BDGREs, level drops did occur with the 5/4/96 and 12/2/98 BDGREs. Thus, the level drop from 1/2/04 to 1/6/04 may indicate a BDGRE.

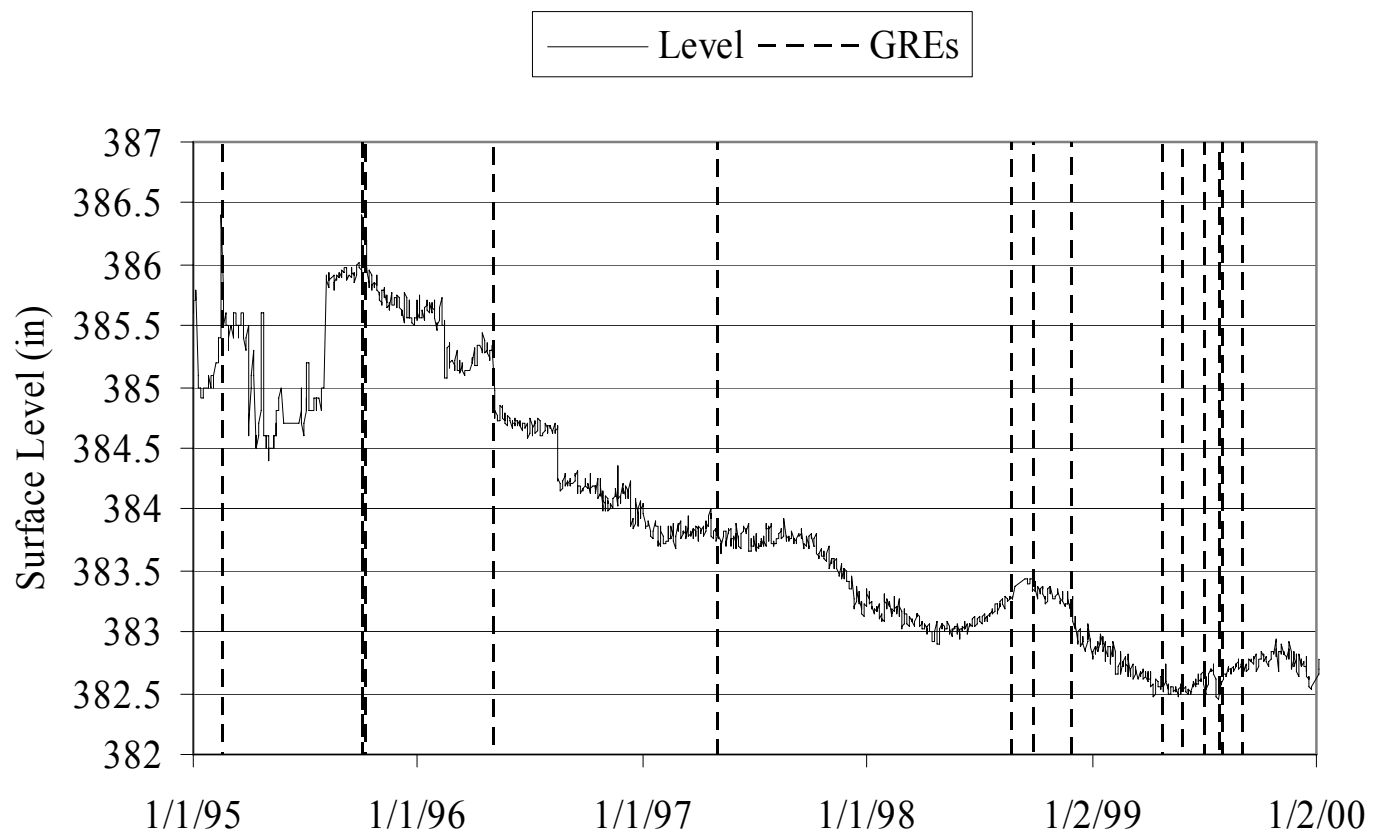

Figure 3.28. AN-104 Surface Level and GRE History, 1/95-1/00 
Level - - - GREs

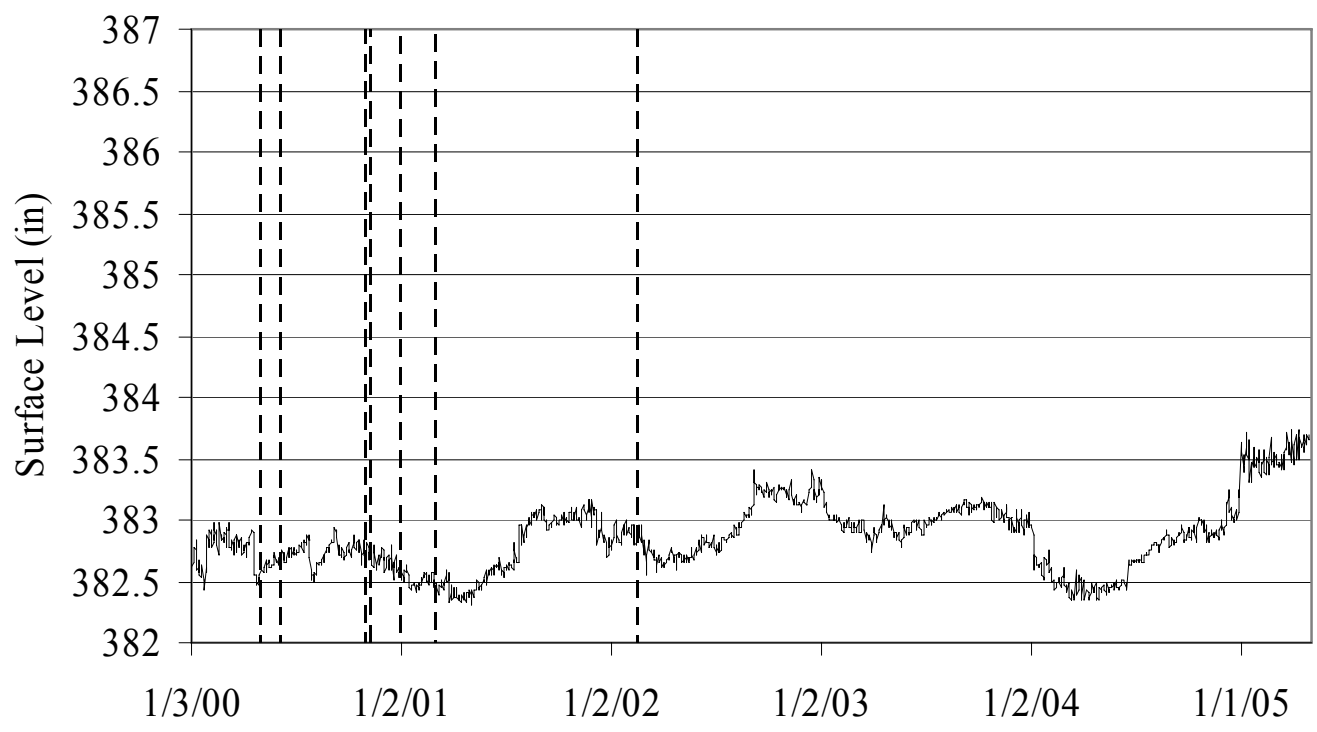

Figure 3.29. AN-104 Surface Level and GRE History, 1/00-4/05

The waste surface in AN-105 has historically had a marked response to some large BDGREs (Figures 3.30 and 3.31; see Table 3.8 for BDGRE size). Level behavior peculiar to AN-105 was an apparent sharp rise followed at a later date by a BDGRE. The numerous small BDGREs in 2000 and 2001 do not appear in the surface level history, but a sizable drop occurred within three weeks of the large release of $6 / 24 / 01$. Thus, keeping in mind the qualitative nature of the surface level response, the surface level drops shown in Figure 3.31 (on 3/24/03, 4/25/03, 8/6/03, 12/22/03, 6/15/04, and 7/18/04) may indicate larger BDGREs than those of 2000 and 2001. The sharp level increases noted on earlier BDGREs are not evident. There is no notable change in the general surface level trend.

Like AN-103 and AN-104, the waste surface level in AW-101 has typically not shown a response to BDGREs (Figure 3.32). Multiple BDGREs occurred in AW-101 from 1995 through mid-1996. After that, the level changed from relatively constant to cyclic (Figures 3.32 and 3.33. Although the level continued to trend up and down after 2001, the behavior was less cyclic. Sudden level drops occurred on 10/5/04 and 3/9/05. These observations may suggest BDGRE behavior is continuing in $\mathrm{AW}-101$, and it may be becoming more frequent (less cyclic) and larger (level drops observed).

The possible BDGRE behavioral changes discussed for AN-103, AN-104, AN-105, and AW-101, which may be attributable to changing tank conditions, may be supported tenuously by 


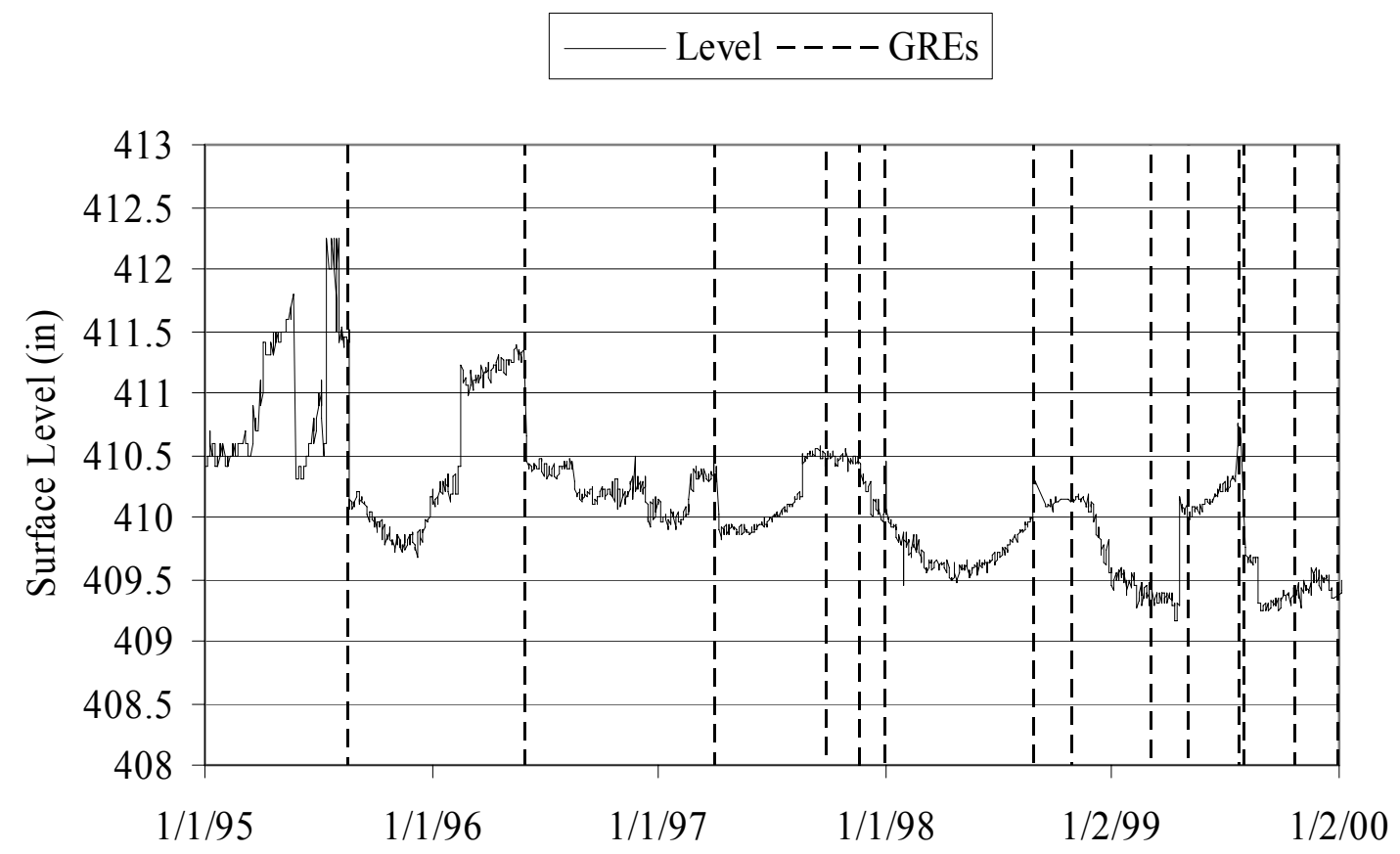

Figure 3.30. AN-105 Surface Level and GRE History, 1/95-1/00

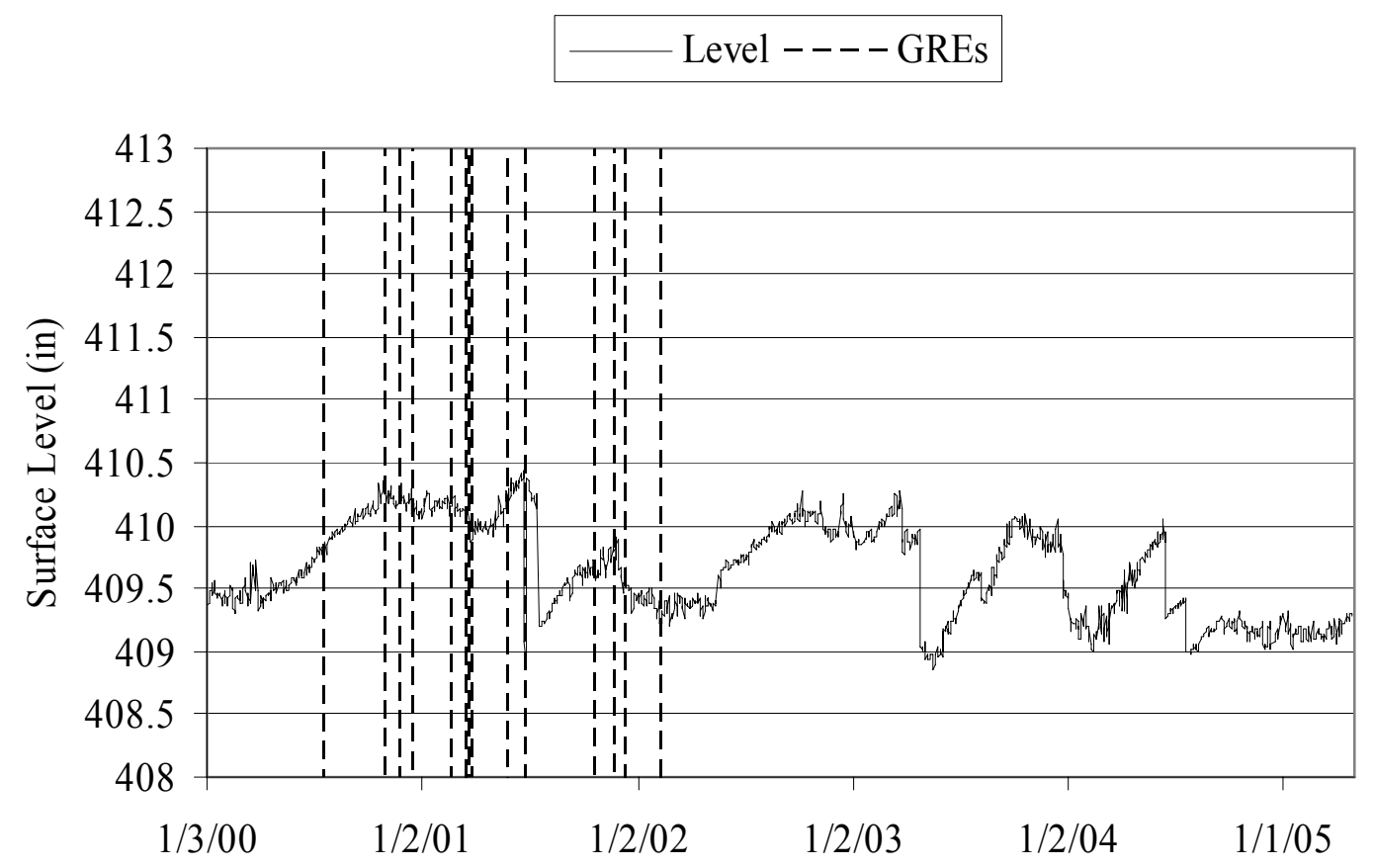

Figure 3.31. AN-105 Surface Level and GRE History, 1/00-4/05 


\section{Level - - - GREs}

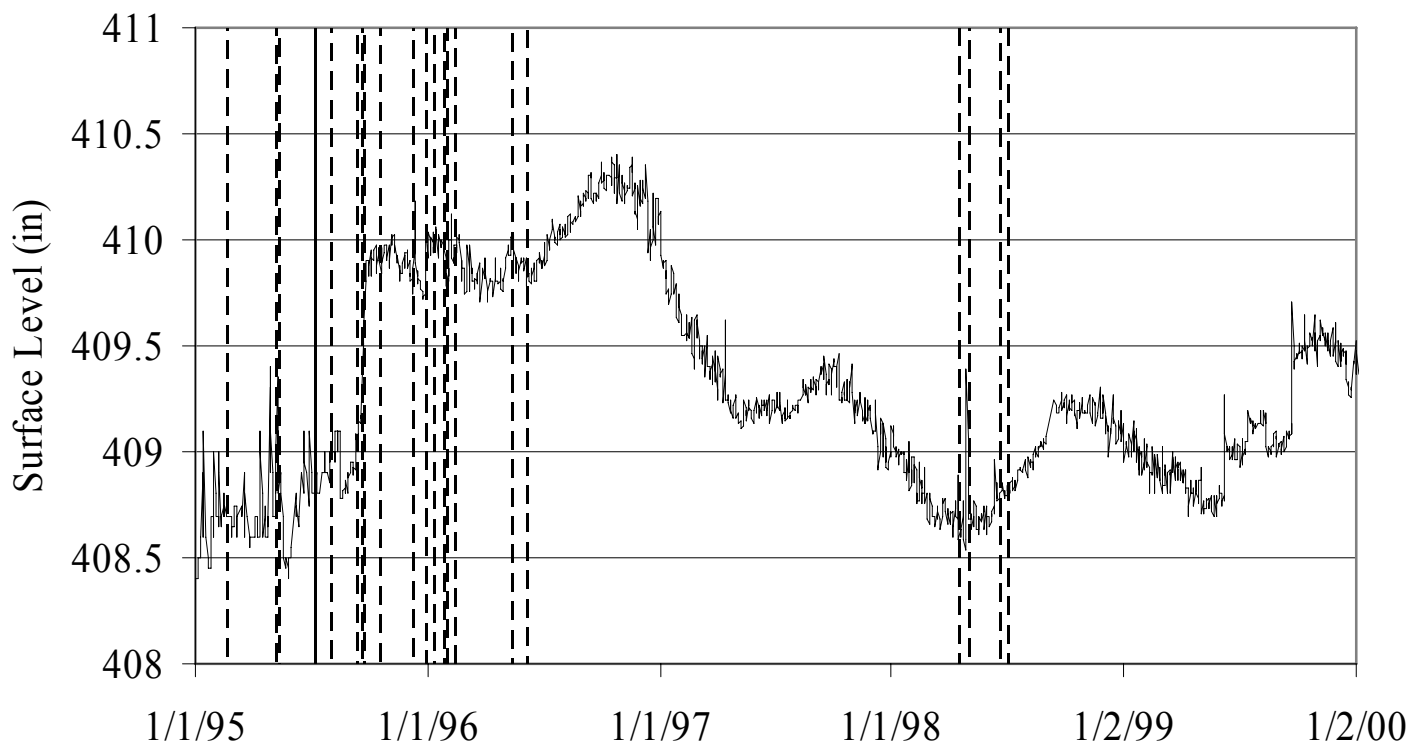

Figure 3.32. AW-101 Surface Level and GRE History, 1/95-1/00

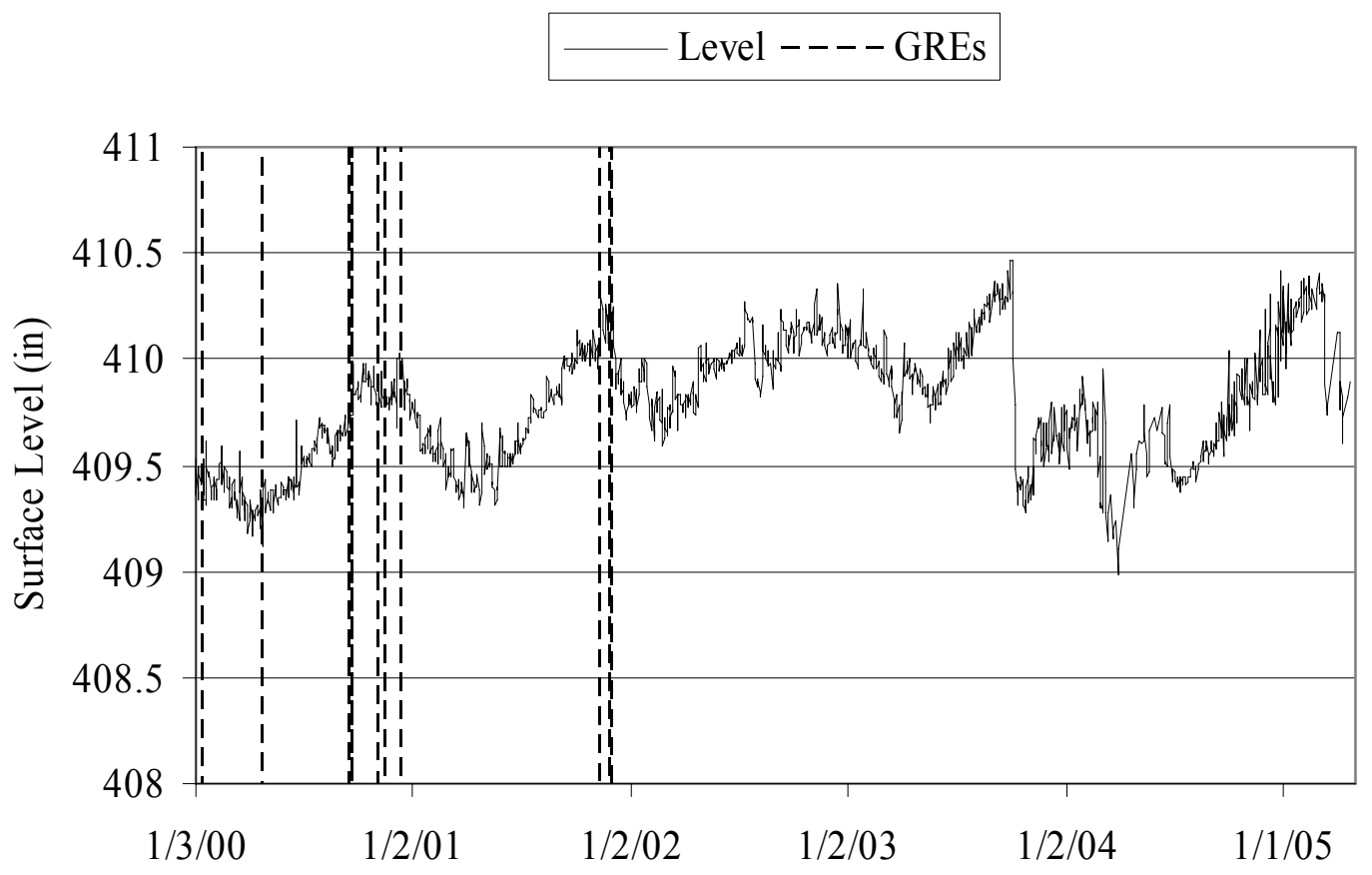

Figure 3.33. AW-101 Surface Level and GRE History, 1/00-4/05 
the observable constant behavior of the relatively unchanged SY-103. (a) The similarity in the level history and BDGRE response for SY-103 is shown in Figures 3.34 and 3.35. The continuing abrupt level changes indicate that BDGRE behavior continues in SY-103. Sharp level increases prior to decreases, similar to those of the large BDGREs in AN-105, are noted.

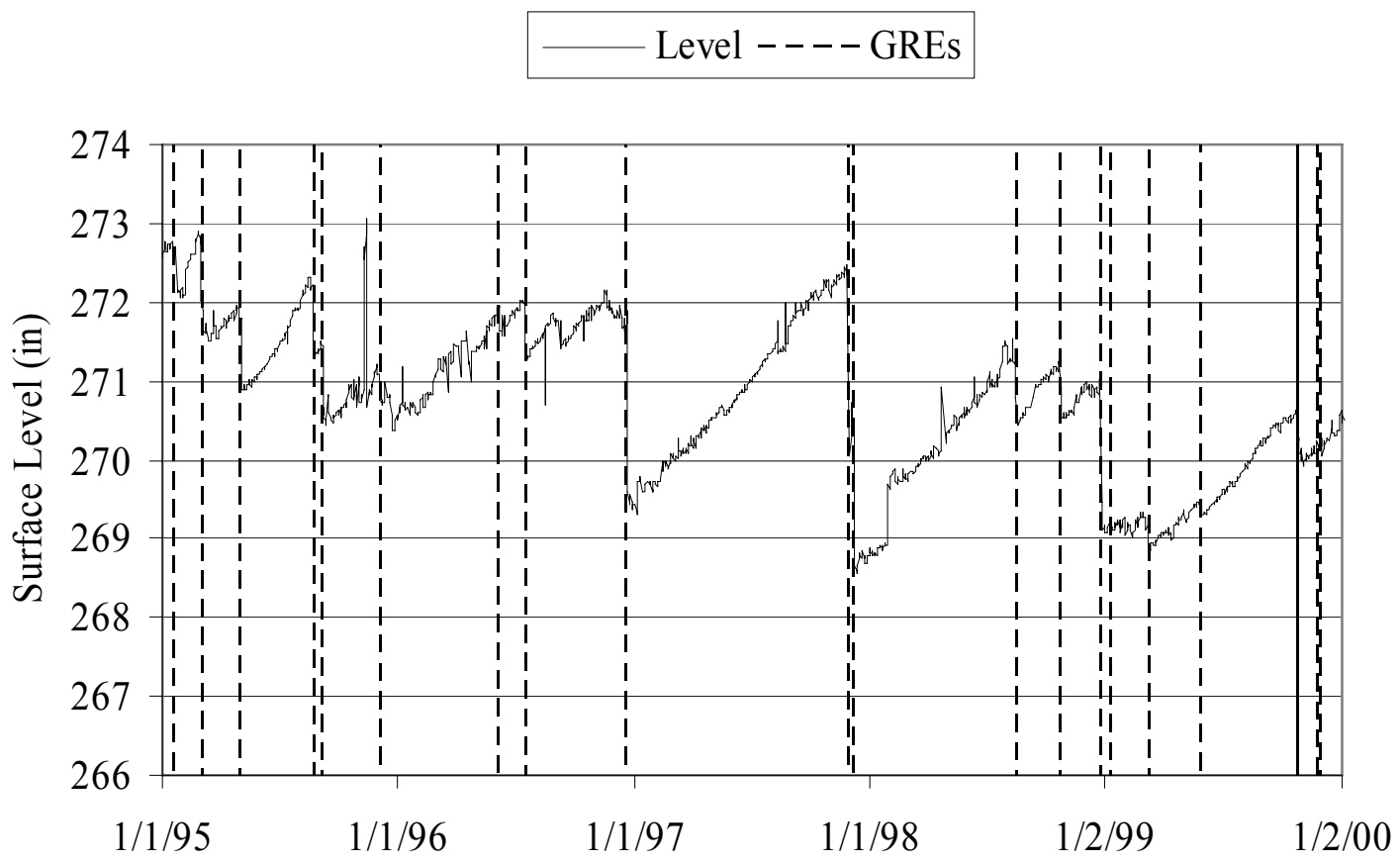

Figure 3.34. SY-103 Surface Level and GRE History, 1/95-1/00

\subsubsection{Input Data Summary}

This subsection summarizes the input data uncertainty distributions in Tables 3.9 through 3.13. Constant values are listed in Table 3.14. Entries that are not denoted either $\mathrm{H}$ or $\mathrm{C}$ for hot or cold respectively are applied to both tank-states. Liquid layer density distributions are limited by the data's maximum and minimum instead of the standard deviation to control the range into a physically plausible (as dictated by the data) state.

(a) The constant BDGRE behavior in SY-103 (Table 3.2.6), which may be expected from the constant waste conditions, has apparently resulted in constant level behavior. The level in tanks AN-103, AN-104, AN-105, and AW-101 could be expected to be constant if BDGRE behavior had not been altered (Table 3.2.6) by changing waste conditions. 
Level - - - GREs

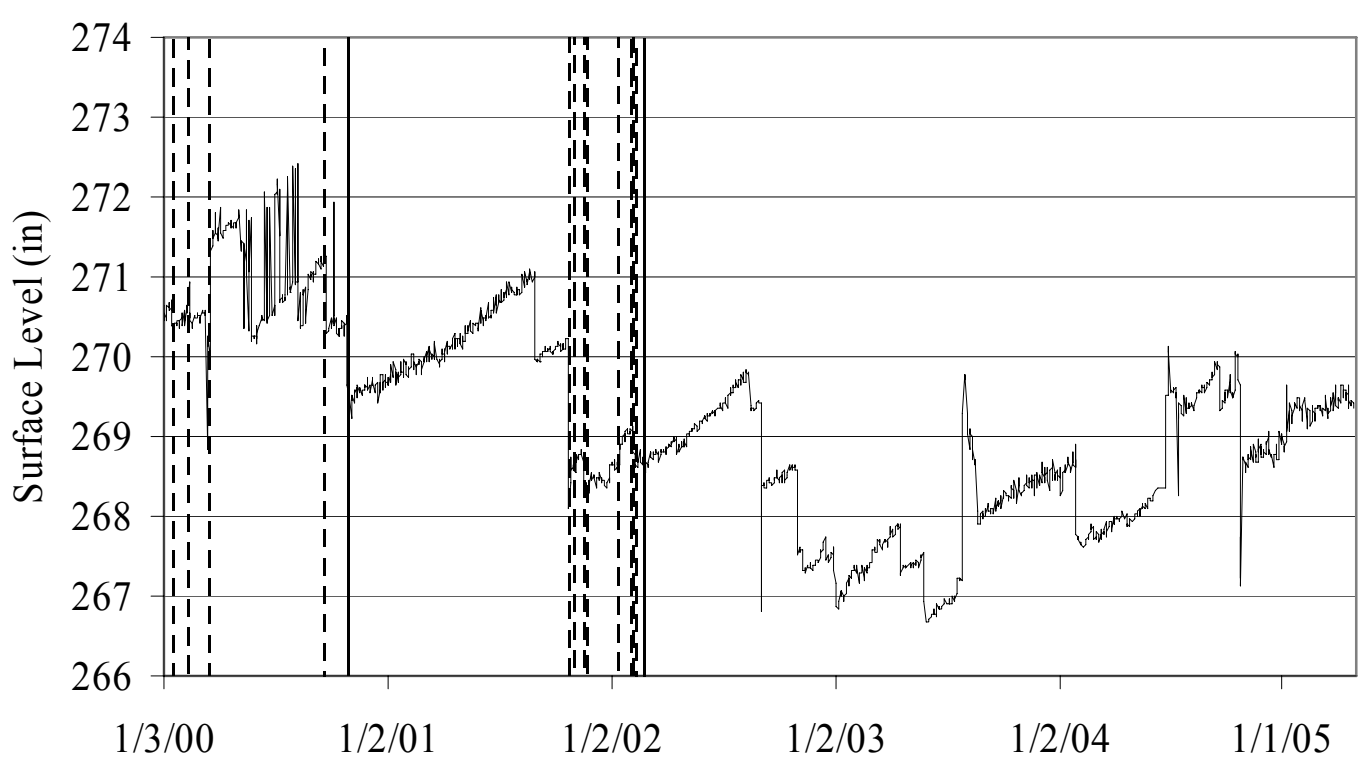

Figure 3.35. SY-103 Surface Level and GRE History, 1/00-4/05

Table 3.9. Liquid Layer Density Uncertainty Distributions $\left(\mathrm{kg} / \mathrm{m}^{3}\right)$ (Section 3.2.3)

\begin{tabular}{|c|c|c|c|c|}
\hline Tank & Median & Mean & Max & Distribution \\
\hline AN-103 & 1,502 & 1,497 & 1,559 & Manipulated Beta \\
\hline AN-104 & 1,408 & 1,403 & 1,500 & Manipulated Beta \\
\hline AN-105 & 1,410 & 1,417 & 1,534 & Manipulated Beta \\
\hline AW-101 & 1,439 & 1,443 & 1,524 & Manipulated Beta \\
\hline SY-101 & 1,490 & 1,488 & 1,559 & Manipulated Beta \\
\hline SY-103 & 1,472 & 1,474 & 1,529 & Manipulated Beta \\
\hline
\end{tabular}

Table 3.10. Sediment Layer Depth Uncertainty Distributions (m) (Section 3.2.4)

\begin{tabular}{|c|c|c|c||}
\hline Tank $^{(a)}$ & Median & Standard Deviation & Distribution \\
\hline AN-103 & 3.787 & 0.29 & Normal \\
\hline AN-104 & 3.954 & 0.31 & Normal \\
\hline AN-105 & 4.360 & 0.154 & Normal \\
\hline AW-101H & 2.687 & 0.287 & Normal \\
\hline AW-101C & 2.950 & 0.287 & Normal \\
\hline SY-101 & 5.33 & 0.395 & Normal \\
\hline SY-103 & 3.273 & 0.29 & Normal \\
\hline (a) "H" or "C" with tank name indicates "hot" or "cold" tank state. \\
\hline
\end{tabular}


Table 3.11. Hydrogen Generation Rate Uncertainty Distributions (mole $/ \mathrm{m}^{3} / \mathrm{d}$ ) (Section 3.2 .5 )

\begin{tabular}{||c|c|c|c|c||}
\hline Tank $^{(\mathrm{a})}$ & Median & Max & Min & Distribution \\
\hline AN-103H & 0.001746 & 0.001921 & 0.000873 & Manipulated Beta \\
\hline AN-103C & 0.000997 & 0.001096 & 0.000498 & Manipulated Beta \\
\hline AN-104H & 0.003122 & 0.003434 & 0.001561 & Manipulated Beta \\
\hline AN-104C & 0.001672 & 0.001840 & 0.000836 & Manipulated Beta \\
\hline AN-105H & 0.001906 & 0.002096 & 0.000953 & Manipulated Beta \\
\hline AN-105C & 0.001276 & 0.001404 & 0.000638 & Manipulated Beta \\
\hline AW-101H & 0.001487 & 0.001635 & 0.000743 & Manipulated Beta \\
\hline AW-101C & 0.001013 & 0.001115 & 0.000507 & Manipulated Beta \\
\hline SY-101 & 0.009700 & 0.011317 & 0.008083 & Normal \\
\hline SY-103 & 0.001909 & 0.002100 & 0.000955 & Manipulated Beta \\
\hline
\end{tabular}

(a) "H" or "C" with tank name indicates "hot" or "cold" tank state.

Table 3.12. Hydrogen Fraction in the Retained Gas Uncertainty Distributions (Section 3.2.6)

\begin{tabular}{|c|c|c|c|c|}
\hline Tank & Median & Max & Min & Distribution \\
\hline AN-103 & 0.61 & 0.687 & 0.533 & Normal \\
\hline AN-104 & 0.45 & 0.519 & 0.381 & Normal \\
\hline AN-105 & 0.59 & 0.644 & 0.536 & Normal \\
\hline AW-101 & 0.32 & 0.352 & 0.288 & Normal \\
\hline SY-103 & 0.46 & 0.687 & 0.27 & Normal \\
\hline SY-101 & 0.29 & 0.31 & 0.27 & Normal \\
\hline
\end{tabular}

Table 3.13. Peak Headspace Hydrogen Concentration Distributions (ppm) (Section 3.2.7)

\begin{tabular}{|c|c|c|c|c||}
\hline Tank $^{\text {(a) }}$ & Median & Mean & 95 $^{\text {th }}$ Percentile & Distribution \\
\hline AN-103C & 510 & 554 & 1,040 & Manipulated Beta \\
\hline AN-104H & 2,169 & 2,412 & 5,013 & Manipulated Beta \\
\hline AN-104C & 241 & 262 & 486 & Manipulated Beta \\
\hline AN-105H & 14,431 & 14,505 & 23,054 & Truncated Normal \\
\hline AN-105C & 1,338 & 1,591 & 3,915 & Manipulated Beta \\
\hline AW-101H & 2,054 & 2,249 & 4,194 & Truncated Normal \\
\hline AW-101C & 502 & 560 & 1,180 & Truncated Normal \\
\hline SY-101 & 27,950 & 28,002 & 52,999 & Truncated Normal \\
\hline SY-103 & 1,020 & 1,141 & 2,564 & Uniform \\
\hline (a) "H" or "C" with tank name indicates "hot" or "cold" tank state. \\
\hline
\end{tabular}


Table 3.14. Constant Input Values

\begin{tabular}{|c|c|c|c|c|}
\hline $\operatorname{Tank}^{(\mathrm{a})}$ & $\begin{array}{c}\text { Sediment } \\
\text { Temperature } \\
(\mathbf{K}) \\
{[\text { Section 3.2.1] }}\end{array}$ & $\begin{array}{c}\text { Sediment Density } \\
\text { for Eq. (3.6) } \\
\left(\mathbf{k g} / \mathbf{m}^{\mathbf{3}}\right) \\
\text { [Section 3.2.2] }\end{array}$ & 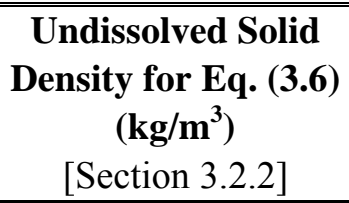 & $\begin{array}{c}\begin{array}{c}\text { Total Waste } \\
\text { Depth } \\
\text { (m) }\end{array} \\
\text { [Section 3.2.3] }\end{array}$ \\
\hline AN-103H & 316 & 1,700 & 2,300 & 8.83 \\
\hline AN-103C & 310 & 1,700 & 2,300 & 8.84 \\
\hline $\mathrm{AN}-104 \mathrm{H}$ & 319 & 1,570 & 2,300 & 9.8 \\
\hline AN-104C & 311 & 1,570 & 2,300 & 9.72 \\
\hline $\mathrm{AN}-105 \mathrm{H}$ & 314 & 1,580 & 2,300 & 10.44 \\
\hline $\mathrm{AN}-105 \mathrm{C}$ & 310 & 1,580 & 2,300 & 10.41 \\
\hline AW-101H & 313 & 1,575 & 2,300 & 10.41 \\
\hline AW-101C & 307 & 1,575 & 2,300 & 10.39 \\
\hline SY-101 & 323 & 1,700 & 2,300 & 10.5 \\
\hline SY-103 & 307 & 1,595 & 2,300 & 6.87 \\
\hline
\end{tabular}




\subsection{Flammability Model and Criteria}

From the discussion in Section 3.1 we concluded that a model based on the buoyancy ratio fit to the data from the hot tank group is the best overall choice to represent the trends in peak hydrogen concentration from BDGREs. The buoyancy ratio is simple, suitable, familiar, and appropriate to the amount and quality of data available. The model is a fit of the (BR-1) $\left[\mathrm{H}_{2}\right]_{\text {gas }} / \mathrm{V}_{\mathrm{HS}}$ quantity to the peak hydrogen concentrations measured during GREs, including the effects of uncertainties in the tank data.

\subsection{Monte Carlo Analysis Results}

Performing 10,000 calculations with input values selected from their respective distributions described in Section 3.2 creates distributions of the output buoyancy ratio for each tank. The resulting cumulative distributions of (BR-1) for the hot tanks are plotted in Figure 4.1, and the statistics for the distributions from all tanks are listed in Table 4.1. Both the plot and the table show that the distributions are quite broad. The 95\% confidence interval of (BR-1) for SY-101 is from 2.2 to 6.4 and in SY-103 from -0.1 to 1.8 . The median is generally skewed toward the lower values.

The final flammability criterion must account for both the uncertainty in the buoyancy ratio calculation described by the Monte Carlo simulation results and the variation of BDGRE size. It is neither practical nor proper to model all 10,000 Monte Carlo outputs for each of the six hot tanks. It is necessary to randomly choose the smaller number that represents the population to get a meaningful model. If all 10,000 data points are used, the model will arbitrarily show a low variability and high fit, due to the uncommonly large number of degrees of freedom available for the model (degrees of freedom are associated with the number of records in the data, in this case, 10,000). As the number of records becomes larger, the variability in the model and the data becomes smaller. Because these records were arbitrarily chosen to be 10,000 long, it would not be appropriate to form a model based on all 10,000 (the resulting models would be falsely good). Instead, a more reasonable number of records, 30, were randomly chosen. According to the Central Limit Theorem, the mean of a distribution is asymptotically normally distributed when $\mathrm{n}$, the number of records, is large enough. "Large enough" has been proven to be at least 30 (Mendenhall et al. 1990).

Because the waste conditions under which each BDGRE occurs are described by the same quantities (e.g., density, sediment depth, temperature) the same 30 Monte Carlo output values for the buoyancy ratio are repeated for each BDGRE. This creates a "cloud" of data wherein each tank's BDGRE behavior and conditions are represented by a rectangular region whose vertical dimension is the range of peak hydrogen concentrations for all BDGREs recorded for the tank and whose horizontal dimension is the range of the function $\mathrm{f}(\mathrm{BR})=10^{6}(\mathrm{BR}-1)\left[\mathrm{H}_{2}\right]_{\text {gas }} / \mathrm{VHS}$ of the 30 records selected from the Monte Carlo output (see Figure 4.2). 


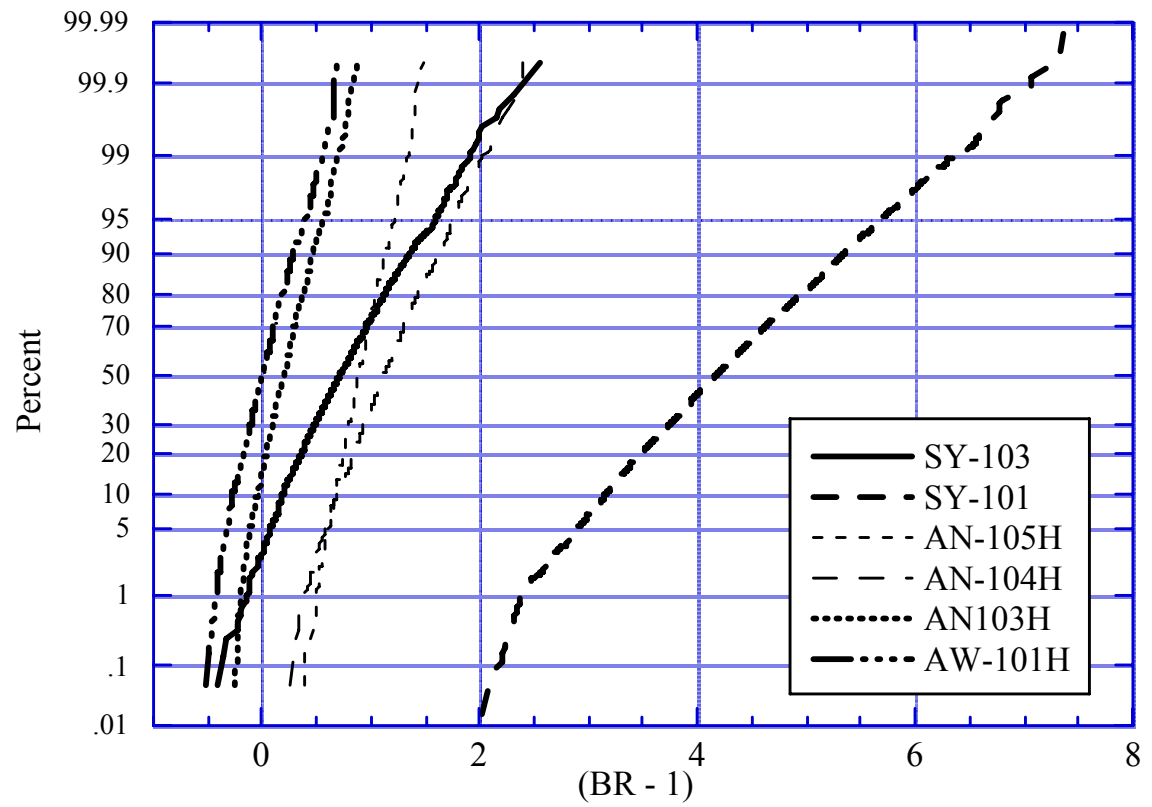

Figure 4.1. Cumulative Distributions of BR-1

Table 4.1. Statistics of BR-1 Output Distributions from the Monte Carlo Simulation

\begin{tabular}{|c|c|c|c|c|c|c|c|}
\hline \multirow{2}{*}{ Tank $^{\text {(a) }}$} & \multirow{2}{*}{ Average } & \multirow{2}{*}{ St. Dev. } & \multirow{2}{*}{ Max. } & \multicolumn{2}{|c|}{ 95\% Confidence Band } & \multirow{2}{*}{ Min. } \\
\cline { 5 - 7 } SY-101 & 4.22 & 0.87 & 7.36 & 6.02 & 4.18 & 2.62 & 1.76 \\
\hline AN-104H & 1.14 & 0.45 & 2.47 & 1.87 & 1.13 & 0.49 & 0.14 \\
\hline AN-105H & 0.90 & 0.18 & 1.62 & 1.27 & 0.89 & 0.56 & 0.32 \\
\hline SY-103 & 0.75 & 0.45 & 3.27 & 0.48 & 0.71 & -0.38 & -0.42 \\
\hline AN-103H & 0.20 & 0.20 & 0.99 & 0.61 & 0.19 & -0.16 & -0.38 \\
\hline AW-101H & 0.01 & 0.22 & 0.88 & 0.48 & 0.00 & -0.38 & -0.57 \\
\hline AN-104C & 0.72 & 0.29 & 1.74 & 1.33 & 0.71 & 0.20 & -0.10 \\
\hline AN-105C & 0.65 & 0.16 & 1.28 & 0.98 & 0.65 & 0.36 & 0.14 \\
\hline AW101C & 0.07 & 0.21 & 0.86 & 0.52 & 0.05 & -0.31 & -0.51 \\
\hline AN-103C & -0.01 & 0.16 & 0.64 & 0.33 & -0.02 & -0.31 & -0.47 \\
\hline (a) "H" or "C" with tank name indicates "hot" or "cold" state. \\
\hline
\end{tabular}

Fitting a model to this cloud of data would provide estimates of the mean value of the peak BDGRE hydrogen concentration as a function of $\mathrm{f}(\mathrm{BR})=10^{6}(\mathrm{BR}-1)\left[\mathrm{H}_{2}\right]_{\text {gas }} / \mathrm{V}_{\mathrm{HS}}$. But the flammability criterion must be derived around the largest reasonably expected BDGRE, not the average. This value is estimated by fitting a probability distribution to the recorded BDGREs and calculating the 95 percentile value, as described in Section 3.2.7. The headspace volume used for calculating the corresponding $\mathrm{f}(\mathrm{BR})$ value is the average over all BDGREs. The result is a single line of data for each tank representing the $95^{\text {th }}$ percentile BDGRE as shown relative to the cloud of all of the BDGRE data for hot and cold tanks in Figure 4.2. 


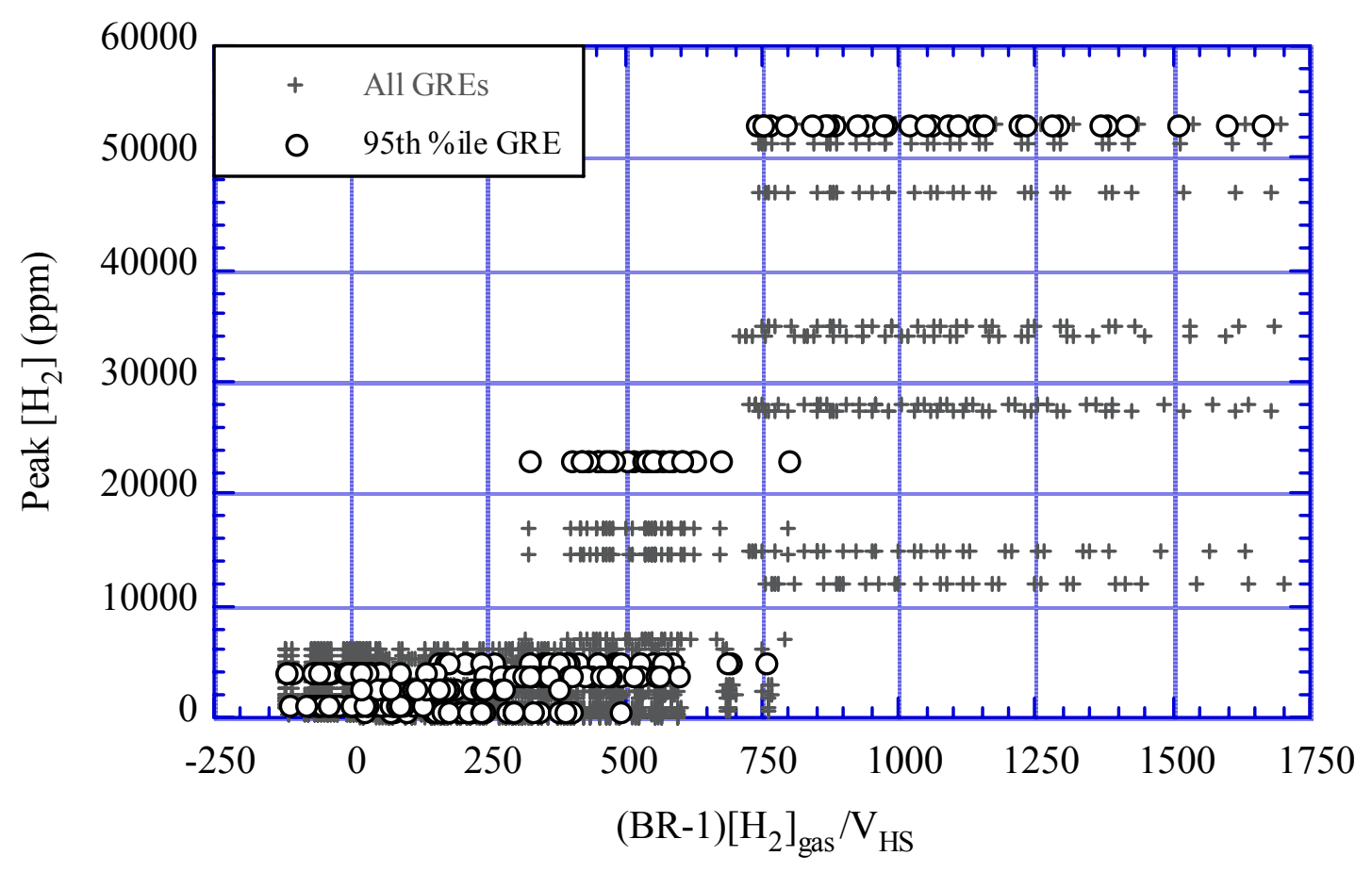

Figure 4.2. $95^{\text {th }}$ Percentile BDGREs Compared to BDGRE Data

To develop a model for the relationship between the $95^{\text {th }}$ percentile of the recorded peak hydrogen concentrations and the quantity $(\mathrm{BR}-1)\left[\mathrm{H}_{2}\right]_{\mathrm{gas}} / \mathrm{V}_{\mathrm{HS}}$, we considered both linear and quadratic functions. The model predictions and the upper and lower bounds of the $95 \%$ confidence interval for the set of values that gave the best fit using data from hot and cold tanks are shown in Figure 4.3. The quadratic model gave the best fit in terms of both $\mathrm{R}^{2}(0.83)$ and RMS error. The linear model had $\mathrm{R}^{2}=0.79$ and $12 \%$ greater RMS error. The quadratic model also captures the $95^{\text {th }}$ percentile BDGRE of SY-101 much better than the linear model.

To ensure that 30 values chosen at random from the Monte Carlo results adequately represent the output distribution of all 10,000 values, the quadratic and linear models were fit with ten sets of 30 different values each for each tank. The resulting range in the quadratic model fits at the upper bound of the 95\% confidence interval is illustrated in Figure 4.4. The best fit occurs for the set of 30 values at the right-hand boundary of the range labeled "minimum" on the figure. This plot illustrates that the most variability in the possible models occurs between the AN-105 and SY-101 data, above 20,000 ppm peak hydrogen concentration. 


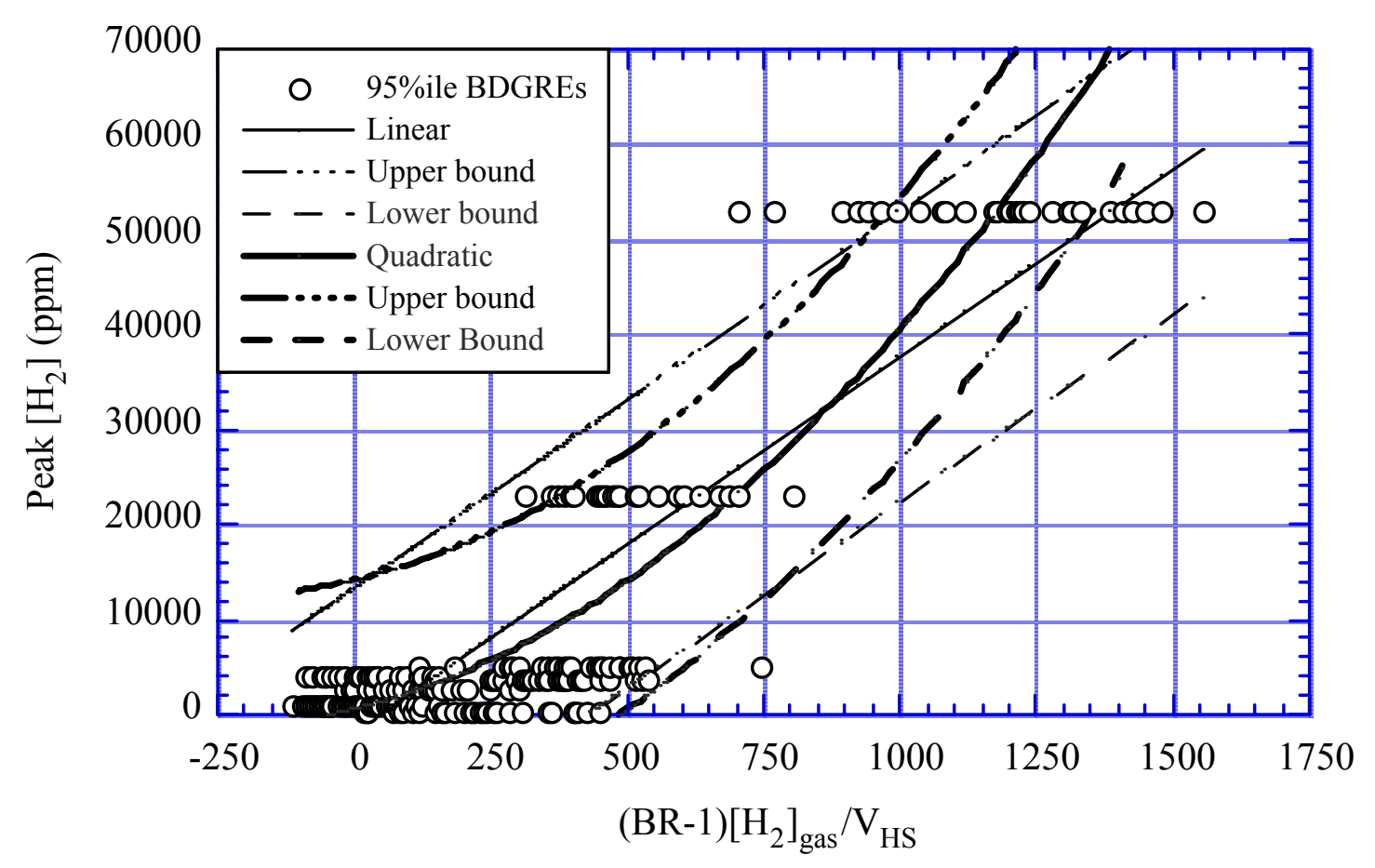

Figure 4.3. Quadratic and Linear Models for $95^{\text {th }}$ Percentile Peak $\left[\mathrm{H}_{2}\right]$

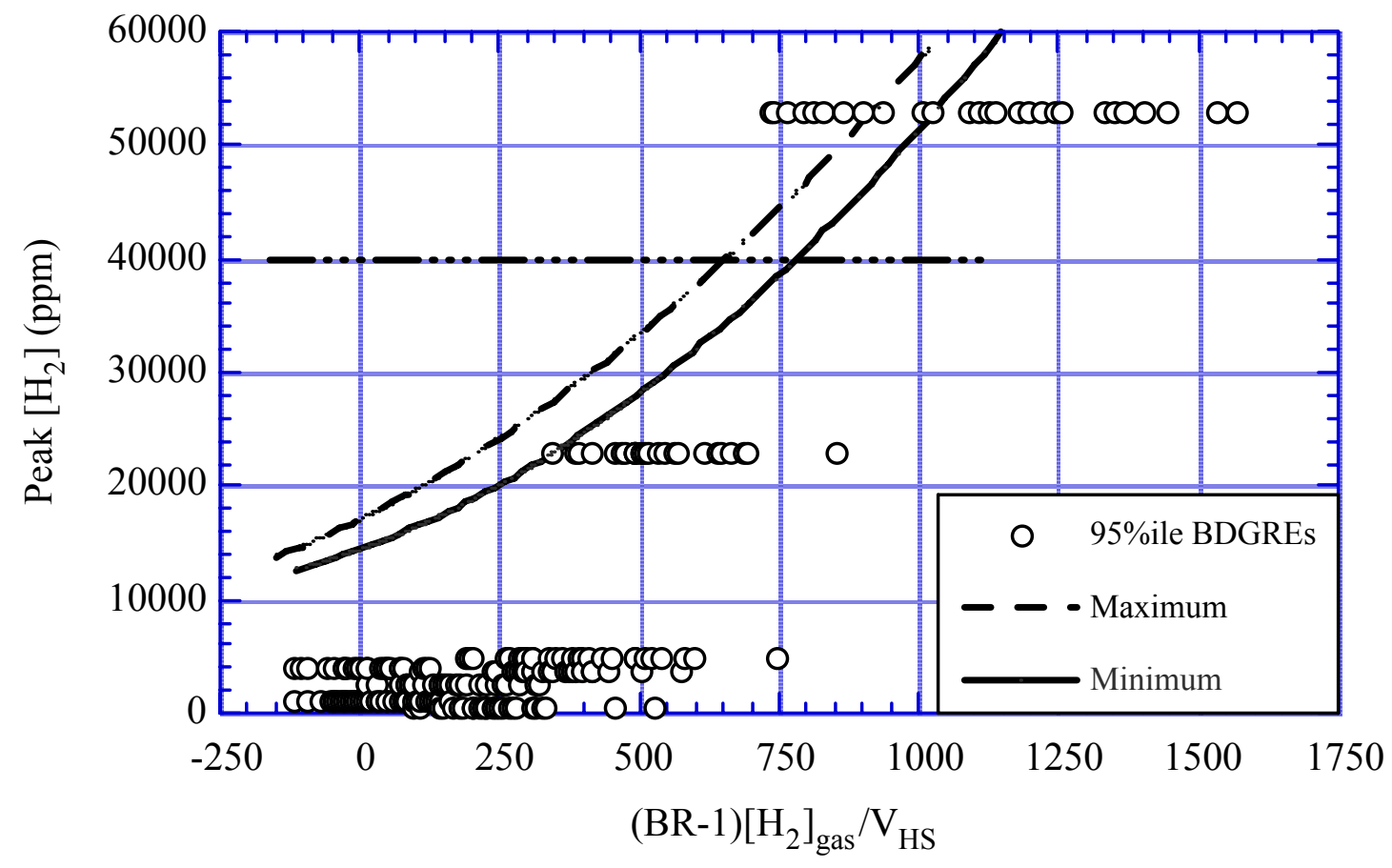

Figure 4.4. Range of Quadratic Models at Upper Bound of the $95 \%$ Confidence Interval 


\subsection{Limiting Buoyancy Ratio Criterion}

The limiting buoyancy ratio criterion is derived from the upper bound of the $95 \%$ confidence interval of the best quadratic model fit to the $95^{\text {th }}$ percentile peak hydrogen concentration estimates shown in Figure 4.3. The equation describing the upper bound of the $95 \%$ confidence interval of the quadratic model for the best set of 30 Monte Carlo output values, which represents the best fit, is given by

$$
0.01895 f(B R)^{2}+18.01 f(B R)+14432=\left[\mathrm{H}_{2}\right]_{\text {peak }}
$$

where $\mathrm{f}(\mathrm{BR})=10^{6}(\mathrm{BR}-1)\left[\mathrm{H}_{2}\right]_{\mathrm{gas}} / \mathrm{V}_{\mathrm{HS}}$. Solving Eq. (4.1) for the buoyancy ratio gives

$$
\mathrm{BR}=1+\frac{\mathrm{V}_{\mathrm{HS}}}{3.79\left(10^{4}\right)\left[\mathrm{H}_{2}\right]_{\text {gas }}}\left\{-18.01+\sqrt{324.18-0.0758\left(14432-\left[\mathrm{H}_{2}\right]_{\text {peak }}\right)}\right\}
$$

Eq. (4.2) defines the limiting value of the buoyancy ratio for a given maximum hydrogen concentration $\left[\mathrm{H}_{2}\right]_{\text {peak }}$. The limit can be set to the lower flammability limit (LFL) for hydrogen, $40,000 \mathrm{ppm}$, a value accounting for the presence of other fuel gases calculated via LeChatelier's law, or some lower concentration like 25\% LFL. However, the minimum value for the hydrogen concentration, which gives a buoyancy ratio of 1.0 , is $14,432 \mathrm{ppm}$. Besides the limiting hydrogen concentration, the limiting buoyancy ratio depends on the retained gas hydrogen volume fraction as well as the tank headspace. (a)

Figure 4.5 shows the limiting buoyancy ratio as a function of headspace for a limiting hydrogen concentration of $40,000 \mathrm{ppm}$, the LFL for hydrogen in air, with the retained gas hydrogen concentration set to 0.5 . A headspace of $530 \mathrm{~m}^{3}$ corresponds to a waste level of 460 inches, while a $2,500 \mathrm{~m}^{3}$ headspace represents a 270 -inch waste level. The limiting buoyancy ratio for a headspace of $1,000 \mathrm{~m}^{3}$ is 2.6 from the figure. For comparison, AN-105, with a headspace of $1,050 \mathrm{~m}^{3}$ has a median buoyancy ratio of 1.91 and a $95^{\text {th }}$ percentile BDGRE hydrogen concentration of $23,000 \mathrm{ppm}$.

Eq. (4.2) can be re-cast in terms of the gas release volume, $V_{R}$, by substituting Eq. (1.2) for $\left[\mathrm{H}_{2}\right]_{\text {peak. }}$. The revised equation is

$$
\mathrm{BR}=1+\frac{\mathrm{V}_{\mathrm{HS}}}{3.79\left(10^{4}\right)\left[\mathrm{H}_{2}\right]_{\mathrm{gas}}}\left\{-18.01+\sqrt{324.18-0.0758\left(14432-10^{6}\left[\mathrm{H}_{2}\right]_{\mathrm{gas}} \frac{\mathrm{V}_{\mathrm{R}}}{\mathrm{V}_{\mathrm{HS}}}\right)}\right\}
$$

(a) The retained gas hydrogen fraction is unknown except for the 6 BDGRE tanks and is assigned a distribution with a median value of 0.5 in Barker and Hedengren (2004). 


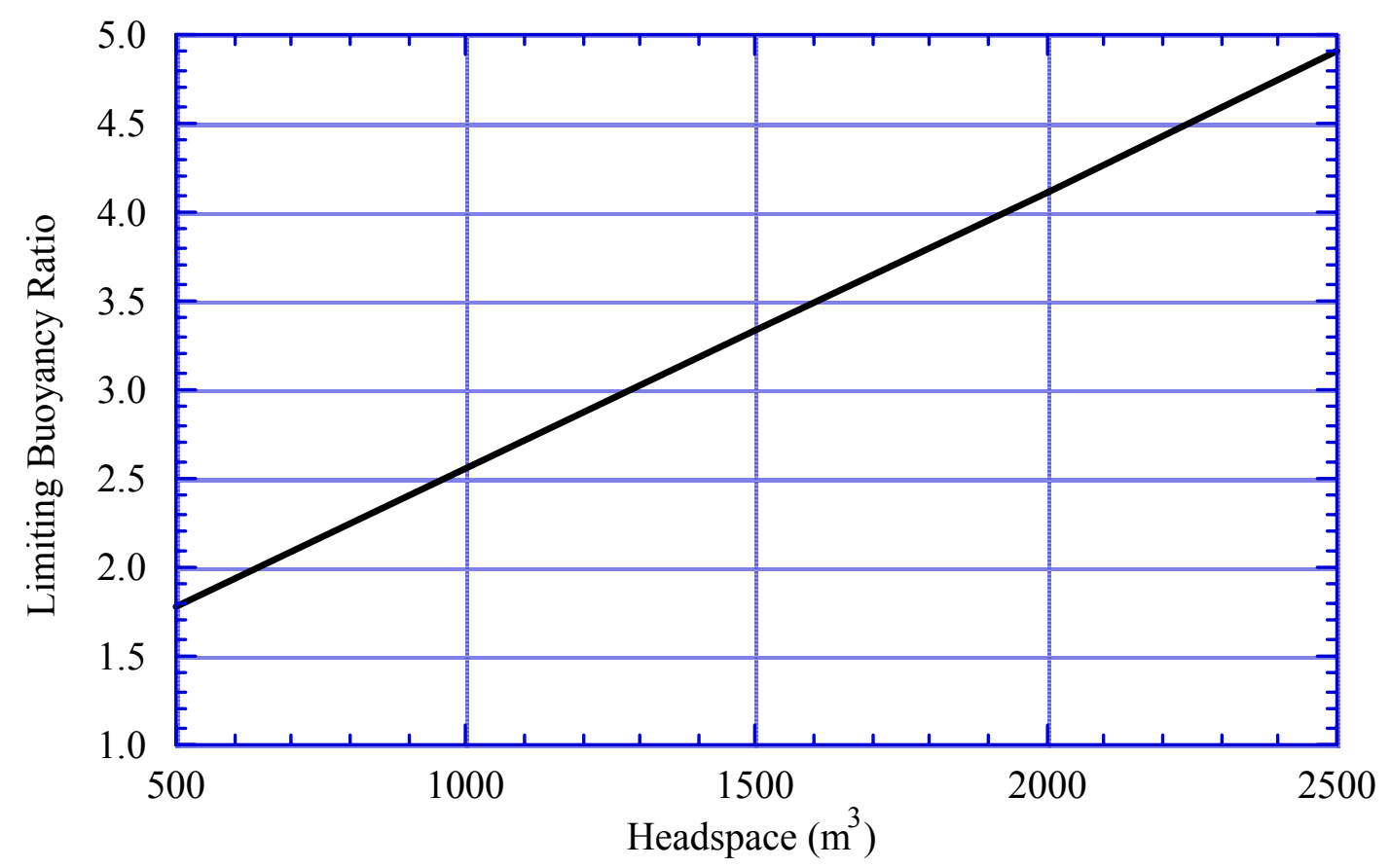

Figure 4.5. Limiting Buoyancy Ratio for $\left[\mathrm{H}_{2}\right]_{\text {peak }}$ of $40,000 \mathrm{ppm}$

\subsection{Recommendations for Applying the Flammability Model}

The limiting buoyancy ratio for specific flammability conditions defined by the tank headspace, retained gas hydrogen fraction, and limiting hydrogen concentration via Eq. (4.2) can be used exactly like the current limit of $\mathrm{BR}<1$ as described by Barker and Hedengren (2004). However, for planning operations, the CH2M HILL specialty assessment recommended a "factor of safety" of 2 be applied to ensure that later adjustments to property values or new measurements will not push the buoyancy ratio above the limit (Kirch and Meacham 2004). This means that the result of Eq. (4.2) should be divided by the factor of safety.

Alternatively, the flammability model of Eq. (4.2) could be established as the safety limit while the original $\mathrm{BR}<1$ condition set as the operating limit. Based on the numerical values of the example shown in Figure 4.5, this would represent a factor of safety of at least 2 except for the smallest headspace volumes.

The derivation of the expression for the limiting buoyancy ratio included the major uncertainties in the tank data as well as variation in BDGRE size, and it would be reasonable to use the median value of the buoyancy ratio calculated for other tanks to compare with the limiting value. However, this requires that the uncertainty range for the other tanks be similar to that of the six BDGRE tanks on which the limit is based. Because the data for these six tanks is likely to be much better defined than other tanks for which fewer measurements are available, the uncertainties of the other tanks are probably larger. Therefore, a larger safety-margin may be 
achieved by using something like the $95^{\text {th }}$ percentile value of the buoyancy ratio calculated in a Monte Carlo simulation to compare against the limit as is currently done (Barker and Hedengren 2004).

The buoyancy ratio for the six BDGRE tanks calculated in developing the limiting criteria uses a leading constant, $\mathrm{C}=21.8293$ in Eq. (1.18). This constant value makes the minimum buoyancy ratio for the six BDGRE tanks exactly equal to 1 (the minimum tank is AN-103 cold) based on an exhaustive evaluation of all available tank data (see Section 3.2). This constant must be used in calculating the buoyancy ratios to be compared with the limit from Eq. (4.2), even though current and future tank inputs give different buoyancy ratios for the BDGRE tanks for which the minimum will not be equal to one.

The most important requirement is that these results not be used in any safety-related calculation without a full understanding of the model basis, data set, and limits of applicability described in Sections 1, 3, and 5. Additionally, to apply the models in accordance with the limitations described in Section 5, the sediment must be characterized in terms of yield stress, the trend of yield stress with depth, and brittleness, or reasonable assumptions made about these properties. 


\subsection{Applicability of the BDGRE Model Suite}

In the ideal world, the range of applicability of a mathematical model of a process can be defined more or less precisely based on the range of data used to develop and validate it and the assumptions made in deriving the equations used. However, the world of gas retention and release in radioactive waste tanks is far from ideal. The data are sparse and uncertain and the physical processes are not fully understood. It is impossible to formally specify a precise range of applicability in terms of tank data values. Nevertheless, the model can be shown to be applicable to specific waste conditions, either because the conditions conform to the model bases or because the model's predictions in some nonconforming conditions are conservative. Likewise, the model is clearly not applicable in other specific cases. This section will provide as much guidance as possible on these boundaries and limitations given the data available. Model validation is discussed in Section 5.1, and its applicability and limitations are described in Section 5.2. Guidance on using tank data to determine model applicability is given in Section 5.3, and conclusions and recommendations are offered in Section 5.4.

\subsection{Validation Status and Prospects}

Formal validation of a mathematical model is normally accomplished by comparing the model's predictions to an independent data set that was not used to develop the model. However, in developing models involving the occurrence or size of BDGREs, the only data available are from six tanks that have historically exhibited BDGREs. (a) These data must be used to derive the model, leaving no independent data to validate it. Theoretically, half the data could be used for development and the other half reserved for validation. However, AN-105H, a very important data set, has only three BDGREs, which cannot reasonably be split in half. Neither is it reasonable to divide the six tanks into two groups. Also, because the same values representing tank conditions must be applied to all BDGREs in that tank, the data are not truly independent.

Large-scale or small-scale simulant tests could theoretically provide additional data on BDGREs. Small-scale experiments, in fact, form the basis for the energy ratio criterion. But realistic experiments that could be claimed to represent the long-term waste behavior treated by the buoyancy ratio model are very difficult or impossible to do.

There are two specific independent applications of the buoyancy ratio that help to validate negative predictions of whether a waste condition would exhibit BDGREs. The buoyancy ratio was evaluated for estimated conditions following transfer of C-106 waste into AY-102. The analysis predicted that AY-102 would not have BDGREs. (b) No BDGREs have been identified

(a) Surface level data imply that other tanks may have exhibited large gas releases in the past, but no gas monitoring data are available to quantify the releases or to assess whether they were, in fact, BDGREs.

(b) Cuta JM, DL Lessor, SA Bryan, CM King, LR Pederson and CW Stewart. July 1999. Assessment of Flammable Gas Retention Potential for WRSS Campaign \#3. Letter Report C106S99.03 Rev. 1, PNNL, Richland, Washington. 
in AY-102 six years after sluicing was completed. The volume of transfer and water dilution necessary to stop BDGREs in SY-101 was estimated based on the buoyancy ratio among other considerations (Johnson et al. 2000). The buoyancy ratio calculated from estimated and later on measured waste conditions after remediation predicted that SY-101 would be a non-BDGRE tank, and it apparently remains so after five years.

In addition to these two tanks where conditions were drastically changed over a short period, effectively creating a "new" tank for a fresh evaluation, there are many existing tanks that have been in a nearly constant state (except for the gradual cooling from radiation decay) for a decade or more. The bona fide non-BDGRE tanks in this group all have buoyancy ratios less than unity, again validating the negative prediction of the model. These tanks include some with much higher specific gas generation rates than SY-101 though with shallow sludge sediment (AY-101, AZ-101, and AZ-102) and tanks with sediments almost as deep as AW-101 but consisting of sludge rather than saltcake and having low gas generation rates (AW-103, AW-106, and AW-105), as well as a few with moderate gas generation and relatively low neutral buoyancy gas fraction (AW-104 and AN-102). At the same time, it is pointed out in Section 5.2 that many or most of these tanks fall outside the specific technical or empirical basis of the models.

On the other hand, the data listed for AN-107 indicate that BDGREs should occur but apparently have not.(a) AN-107 has a relatively high estimated gas generation rate due to his high total organic carbon (TOC) concentration. A small difference between the liquid and sediment densities creates a very low neutral buoyancy gas volume fraction. These two effects predict a buoyancy ratio exceeding unity. The AN-107 density data are discussed in Appendix E. Similarly, in the cold condition of Tank AW-101, an increase in sediment depth overcompensates for the reduction in gas generation rate due to lower waste temperatures, actually producing a slightly higher buoyancy ratio than in hot condition. However, the recorded gas releases, while gas monitoring data was being recorded up to September 2002, were much smaller in the cold tank.

In summary, the buoyancy ratio model has successfully predicted that BDGREs would not occur as a result of major operations in two tanks, and all but one of the existing non-BDGRE tanks have buoyancy ratios much less than unity. The one tank predicted to have BDGREs apparently has not had any, and another tank predicted to have larger BDGREs has had small ones. There have been no cases where BDGREs occurred in a tank with a buoyancy ratio less than unity. Therefore, while a decade of tank experience has not formally validated the model, neither has it been disqualified. The validity of the buoyancy ratio must rest on how it separates the tanks in which BDGREs are observed from the others and how its magnitude represents the size of the observed BDGREs. Formal validation is simply not possible with existing data.

(a) No gas monitoring data are available for the period where BR $>1$ in AN-107, but there have been no indications of large BDGREs in its recent level history. 


\subsection{General Applicability Assessment}

To the knowledge of the authors, this is the first detailed, focused analysis of the applicability of these models. We recognize that the buoyancy ratio and energy ratio models have been used for many years without specific case-by-case determination of applicability. They were used because they were available, based on models derived from plausible physical mechanisms, and correctly identified the existing BDGRE and non-BDGRE tanks. Detailed information on waste physical properties was available only for the BDGRE tanks, and, in the absence of data to the contrary, the other tanks with similar waste configurations were assumed to have similar properties.

Recent information casts doubt on some of our original assumptions about waste properties in tanks where such data were not initially available. Nevertheless, the most certain range of applicability of the models is still represented by the tanks currently exhibiting BDGREs, and potentially hazardous waste conditions that might produce BDGREs are also most likely to resemble these tanks. Thus, the database from which these models were developed covers the conditions where their application is likely to be most important.

Over the next decade, the necessities of double-shell tank waste management will create new tanks with waste conditions and properties different from the current BDGRE tanks. Retrieval of single-shell tank waste into the double-shell tanks will create distinct layers of waste with different properties. The need to fill tanks to higher levels with increasingly concentrated waste may create conditions different than those of the current BDGRE tanks.

While these changes are creating tanks with ever more widely varying conditions, the range of data representing the six BDGRE tanks is quite narrow. Their waste physical properties are very similar (Hedengren et al. 2000). The sediment yield stress is relatively low, increasing from zero at the top to a few hundred Pascals. The difference between sediment and liquid densities is small and the waste composition represents concentrated saltcake. While there is not yet definitive or complete data, an increasing number of observations indicate that sludge sediments may have a much higher yield stress, on the order of 1,000 $\mathrm{Pa}$, and be more brittle than previously assumed.

The waste configurations of the six BDGRE tanks cover a wider range that is probably adequate to represent most conditions created by retrieval and other operations. The sediment depth varies by more than a factor of 2 from 2.7 to $5.8 \mathrm{~m}$, and the total waste depth ranges from 6.9 to $10.5 \mathrm{~m}$ with headspace volumes from 1,050 to $2,500 \mathrm{~m}^{3}$. The sediment was initially added in layers, as anticipated for future transfers, including a relatively strong, nonuniform heel on the bottom. The calculated total gas generation rates differ by about an order of magnitude, from 0.003 to $0.033 \mathrm{~mole} / \mathrm{m}^{3}-\mathrm{d}$. However, the high value is unique to SY-101. The next highest is AN-104 with $0.007 \mathrm{~mole} / \mathrm{m}^{3}-\mathrm{d}$. 
The discussions in this section apply to both the use of the buoyancy ratio to identify BDGRE tanks and the use of buoyancy ratio-based flammability model and criteria described in Section 4.2 to assess whether a tank has the potential to reach a limiting hydrogen concentration (i.e., 40,000 ppm hydrogen) as a result of spontaneous BDGREs. The evaluation of both applications of the buoyancy ratio assumes sufficient depth of supernatant liquid to allow an efficient gas release via an energy ratio screening. The limitations of the energy ratio model and its gas release criterion are also discussed.

\subsubsection{BDGRE Model Summary}

This section briefly summarizes the basis and major assumptions of the buoyancy ratio model, the buoyancy ratio based flammability model, and the energy ratio. The flammability model derivation is described in Section 4.2 and the details of the buoyancy ratio and energy ratio are given in Section 1.

\subsubsection{Buoyancy Ratio Basis and Assumptions}

The buoyancy ratio model addresses only spontaneous BDGREs and gives no information on gas releases other than BDGREs. (a) It is built around the characteristics of the six BDGRE tanks as revealed by core samples and measurements by the ball rheometer and void fraction instrument. The tanks are AN-103, AN-104, AN-105, AW-101, SY-103, and SY-101, and their properties are listed in Section 3.2.8. The buoyancy ratio criterion that states BDGREs are possible for $\mathrm{BR} \geq 1$ also is based on the behavior of these six BDGRE tanks. The criterion is realized by adjusting the leading coefficient of the model Eq. (1.18) so the minimum buoyancy ratio calculated for these tanks is exactly 1.0 .

Variations in the waste layer depth, density, temperature, and gas generation rate are explicitly included in the model equation. Except for the yield stress, the properties of sediment layer are assumed homogeneous. The yield stress is assumed sufficiently low and the sediment sufficiently ductile that gas is retained as discrete, round bubbles. The yield stress is also assumed to increase linearly with depth from zero at the top of the sediment. The equation for the gas volume profile that is integrated to form the buoyancy ratio represents the steady state, which carries the implicit assumption that the tank has reached that state.

\subsubsection{Flammability Model Basis and Assumptions}

The empirical buoyancy ratio-based flammability model derived in Section 4.2 applies only to the prompt, spontaneous, initial gas release that creates the peak headspace hydrogen

(a) Because the buoyancy ratio implies a specific gas volume fraction profile, is used by Stewart et al. (2003) to estimate the initial quantity of retained gas in studying releases induced by global waste disturbances. This is not a prediction of whether induced BDGREs will occur. 
concentration measured in the six historic BDGRE tanks. This restriction carries with it the assumption that a tank is actively ventilated so that long-term gas accumulation can be ignored. The model gives no information on induced releases.

The range of this application is somewhat narrower than assessing BDGRE potential because the data on BDGRE size comes only from the six tanks in which they historically occur. There is no physical basis in using the buoyancy ratio in a flammability model to predict peak hydrogen concentration. It is solely an empirical model and the data range from which the model was developed determines its applicability.

\subsubsection{Energy Ratio Basis and Assumptions}

The energy ratio is defined as the amount of energy available in the rising gob divided by that required to liquefy a gob of sediment rising during a BDGRE. The energy ratio model was derived under the assumption that trapped gas bubbles are released from a gob undergoing a BDGRE when they expand sufficiently to mobilize the sediment around them. Bubble expansion is assumed to be due to reduced hydrostatic pressure in a rising gob and the material being mobilized is assumed to be ductile so that it deforms to high strains at a relatively constant stress. This assumption about the material properties is the primary source of limitations on the applicability of the energy ratio model and criterion.

The energy ratio criterion for gas release (ER $>3$ ) was derived from small-scale $(27-\mathrm{cm}-$ diameter) experiments in bentonite clay and confirmed by the energy ratio values of the five BDGRE tanks (23-m diameter). This indicates that the energy ratio formulation and criterion is fundamentally independent of scale. Unlike the buoyancy ratio, the energy ratio model and criterion are applicable to both spontaneous and induced BDGREs but give no information for other types of gas release.

The remainder of this section discusses the theoretical and empirical basis for applying the buoyancy ratio model, the flammability model, and the energy ratio criterion to a wide variety of waste conditions. The discussion focuses on the large 100-series double-shell waste tanks but extends to some other conditions where appropriate.

\subsubsection{Deep Sediment}

To support retrieval of single-shell tank wastes, the waste levels in the double-shell tanks may be increased as much as possible up to the limit of double-shell containment at 460 inches, presumably with a corresponding increase in sediment depth. The only aspect of waste depth itself that might compromise application of the buoyancy ratio and flammability models would be wall effects. In vessels much taller than their diameter, the wall effect would tend to amplify the influence of the sediment yield stress and increase the excess buoyancy required for a gob to break free. However, with a tank diameter of 900 inches ( 75 feet), wall effects are minimal even 
at the highest reasonable sediment depth. The small effect of increased hydrostatic pressure is included explicitly in the models.

The assumed linear increase in sediment yield stress with depth does imply a high average strength in very deep sediment. If the measured yield stress at the bottom of the 4-m sediment in AN-105 is 200 to $250 \mathrm{~Pa}$ (Hedengren et al. 2000), an 8-m sediment would have a maximum yield stress of $500 \mathrm{~Pa}$. However, this is still probably within the realm of the weak sediment assumption (see Section 5.2.4).

The sediment depth of the quintessential BDGRE tank, SY-101, has been as high as $6 \mathrm{~m}$ (Antoniak 1993). A reasonable upper limit might be the upper bound of the $99 \%$ confidence interval for the sediment depth distribution listed for SY-101 in Table 3.2.8. The distribution is normal with a mean of $5.33 \mathrm{~m}$ and a standard deviation of $0.42 \mathrm{~m}$. The upper bound of the $99 \%$ confidence interval (mean plus three standard deviations) of this distribution is $6.6 \mathrm{~m}$.

This result probably supports the application of both the buoyancy ratio model and the flammability model to sediment layers up $7 \mathrm{~m}$ in depth. However, the energy ratio model is theoretically and empirically independent of scale and is not limited to any maximum sediment depth.

\subsubsection{Uniform Sediment Yield Stress}

A uniform sediment yield stress would likely occur in a material whose strength does not depend directly on the lithostatic load between solid particles, such as clay simulant or strong sediments where the solid particles were bonded together in some manner. Based on available information, sludge may have a uniform or linearly increasing yield stress with depth.

The fundamental assumption of the buoyancy ratio model is that the yield stress increases linearly with depth. However, the gas volume fraction profile and an equivalent buoyancy ratio criterion for a uniform yield stress can be obtained by again deriving the gas volume fraction profile, Eq. (1.17), with a constant sediment yield stress, $\tau_{0}$. This gives the following expression for the gas volume fraction as a function of $\eta=\mathrm{z} / \mathrm{H}_{\mathrm{S}}$ :

$$
\alpha(\eta)=\frac{\tau_{0}}{\mathrm{SK} \rho_{\mathrm{S}} \mathrm{g}} \mathrm{N}^{2 / 3}\left(\frac{\mathrm{RT} \mathrm{S}}{\mathrm{p}_{\text {gas }}}\right)^{1 / 3} \mathrm{G}^{1 / 3} \mathrm{H}_{\mathrm{S}} \eta
$$

Assuming the same bubble migration mechanism and rate expression that produces a parabolic gas volume fraction profile for a linearly increasing yield stress, Eq. (5.1) predicts that the gas volume fraction increases linearly from the bottom with its maximum at the top of the sediment layer. This profile is compared to the parabolic profile of Eq. (1.17) in Figure 5.1. Because the nonbuoyant layer near the top must be supported by excess buoyancy from below, the parabolic profile (left side of the figure) requires most of the sediment to be buoyant if any is 

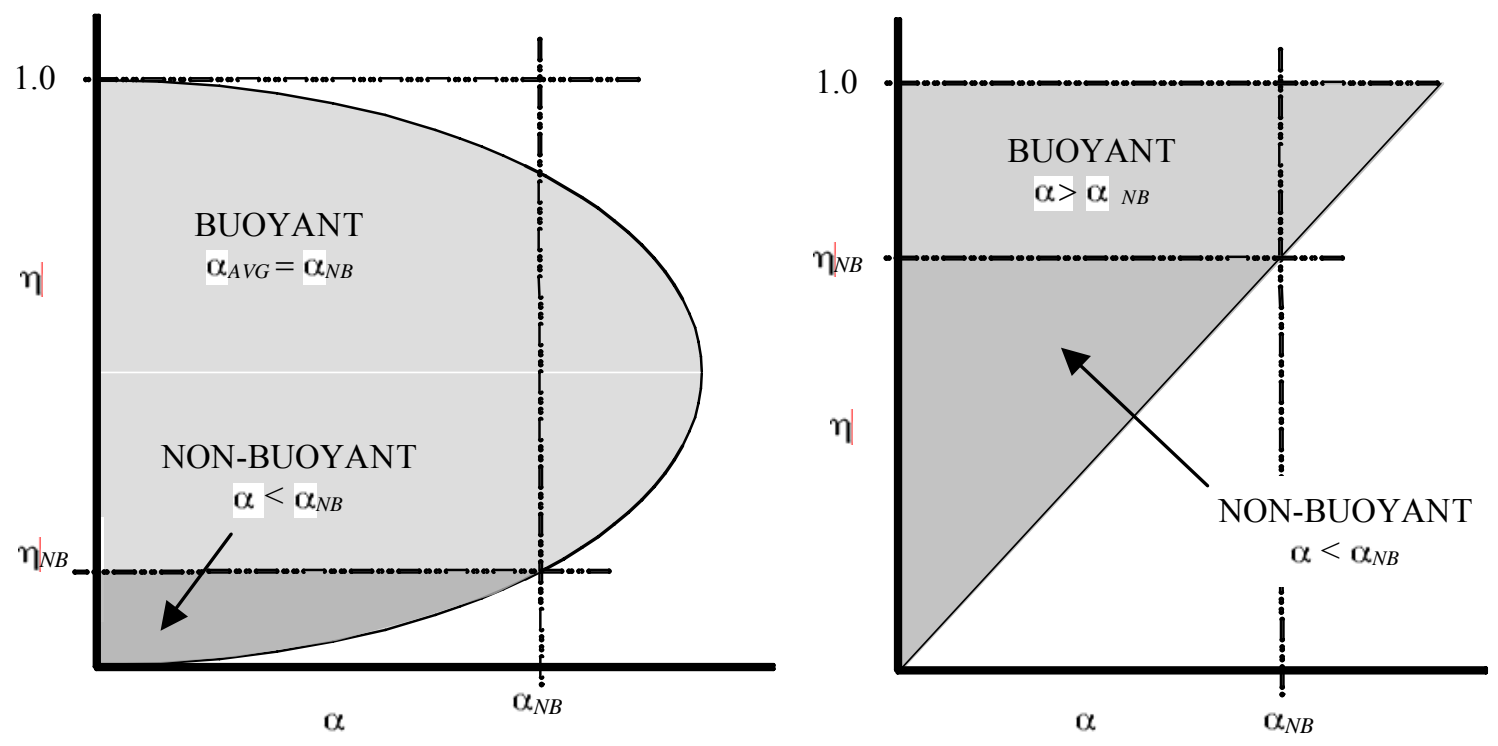

Figure 5.1. Comparison of Gas Fraction Profiles

buoyant. But only a shallow layer at the top is buoyant with a linear profile (right side). In fact, as the profile establishes itself, the first material to become buoyant at the very top of the sediment will release immediately because it has no nonbuoyant overburden to support. However, it is conceptually possible for BDGREs to involve sediment down to the depth where the local gas volume fraction is equal to the neutral buoyancy value.

Because the parabolic profile requires the peak gas volume fraction to exceed $\alpha_{N B}$ by a relatively large fraction to achieve net buoyancy, use of the buoyancy ratio to determine whether BDGREs can occur in sediment with a uniform yield stress is technically nonconservative, though any unpredicted BDGREs will be small.(a) However, no BDGREs can occur at all if the maximum gas volume fraction is less than neutral buoyancy, and the buoyancy ratio limit can be adjusted downward to ensure this.

The maximum gas volume fraction for a uniform yield stress, $\alpha_{\max , \mathrm{U}}$, can be related to that produced by a linearly increasing yield stress, $\alpha_{\max , \mathrm{L}}$, by dividing Eq. (5.1) evaluated at $\eta=1$, where the peak gas volume fraction occurs, by Eq. (1.17) evaluated at $\eta=1 / 2$, where the peak occurs in the parabolic profile, and defining the average yield stress, $\tau_{\text {avg }}=\mathrm{m}_{\tau} \mathrm{H}_{\mathrm{S}} / 2$. The resulting expression is

$$
\alpha_{\max , \mathrm{U}}=\alpha_{\max , \mathrm{L}} \frac{4 \tau_{0}}{\mathrm{H}_{\mathrm{S}} \mathrm{m}_{\tau}}=\alpha_{\max , \mathrm{L}} \frac{2 \tau_{0}}{\tau_{\mathrm{avg}}}
$$

(a) It is not reasonable to postulate a large BDGRE involving a thin layer across a wide area because of natural nonuniformities in waste conditions (e.g., due to temperature distribution). 
A value of $150 \mathrm{~Pa}$ for $\tau_{\text {avg }}$ in Eq. (5.2) represents the six BDGRE tanks. Dividing Eq. (5.2) by $\alpha_{N B}$ and replacing $\alpha_{\max , L}$ with the combination of Eq. (1.18) and Eq. (1.17) evaluated at $\eta=1 / 2$ gives the following expression relating $\alpha_{\max , \mathrm{U}}$ to the buoyancy ratio:

$$
\frac{\alpha_{\mathrm{max}, \mathrm{U}}}{\alpha_{\mathrm{NB}}}=\frac{8}{3} \mathrm{BR} \frac{\tau_{0}}{\tau_{\mathrm{avg}}}
$$

Therefore, to ensure that $\alpha_{\max , U}<\alpha_{N B}$ so that no BDGREs occur in a sediment with uniform yield stress, the limiting buoyancy ratio is given by solving Eq. (5.3) with $\left(\alpha_{\max , U} \backslash \alpha_{N B}\right)=1$ for the BR to give

$$
\mathrm{BR}<\frac{3}{8} \frac{\tau_{\mathrm{avg}}}{\tau_{0}}
$$

Though Eq. (5.4) provides a theoretical basis to apply the original buoyancy ratio model, it is obviously much more restrictive than the $\mathrm{BR}<1$ criterion for waste where the yield stress increases linearly with depth, especially if the uniform yield stress is higher than $\tau_{\text {avg }}(150 \mathrm{~Pa})$. However, it is neither important nor useful to plan operations using Eq. (5.4) to prevent the inconsequential BDGREs resulting from a uniform yield stress. The flammability model provides a more meaningful limit.

Because it is based on the largest observed BDGREs in each tank and because it includes all the important uncertainties in computing the buoyancy ratio, the peak hydrogen concentration predicted by the flammability model is already extremely conservative for the tanks it is based on. The peak hydrogen concentration predicted for a tank with a buoyancy ratio around 1.0 is over $14,000 \mathrm{ppm}$, while the maximum observed for any tank with this low a buoyancy ratio is $6,200 \mathrm{ppm}$ (AW-101 on 10/4/94). Because the linear gas volume fraction profile in tanks with a uniform yield stress demands much smaller BDGREs than from a parabolic profile, a buoyancy ratio of 1.0 would be even more conservative for these tanks in terms of the size of the release.

Therefore, by considering the conservatism of the flammability model, it is clear that the buoyancy ratio model and criterion ensures that no hazardous releases will occur in sediment with uniform yield stress, though small BDGREs will occur at BR $<1$.

The energy ratio model depends only on the average yield stress and is not influenced by its distribution. In fact, the energy ratio criterion was developed from experiments using a simulant with uniform yield stress and is fully applicable to ductile sediments with uniform yield stress.

\subsubsection{Strong Sediment}

Perhaps the most fundamental assumption in analyzing gas retention and release in tanks subject to BDGREs is that the sediment is relatively weak and fluid-like. This assumption 
defines the shape and motion of bubbles migrating through the sediment and how they escape from the sediment during a BDGRE. It provides a physical basis for relating the speed of a bubble to the sediment yield stress and to the gas generation rate through the growing size of the rising bubbles. A strong, structurally robust sediment violates these basic assumptions and is outside the theoretical basis of the models. Nevertheless, the evidence indicates they are conservative under specific conditions.

\subsubsection{Buoyancy Ratio Model in Strong Sediment}

One favorable aspect of a higher waste strength is that the additional buoyancy required by a gob to break free of the surrounding material makes the buoyancy ratio conservative. This overbuoyancy requirement can be ignored in the six BDGRE tanks but becomes more important as the sediment yield stress increases. The critical gas volume fraction, $\alpha_{C}$, required to detach a plane layer of sediment can be computed by (Meyer et al. 1997):

$$
\alpha_{\mathrm{C}}=\alpha_{\mathrm{NB}}+\frac{\beta \tau_{\mathrm{y}}}{\rho_{\mathrm{S}} \mathrm{gH}}
$$

where $1<\beta<\sqrt{3}$ is a factor relating the yield stress in shear to that in tension (the best value appears to be 1.35, as described in Appendix A).

For a sediment $3 \mathrm{~m}$ deep with a density of $1,700 \mathrm{~kg} / \mathrm{m}^{3}$ and $\alpha_{\mathrm{NB}}=11.8 \mathrm{vol} \%, \alpha_{\mathrm{C}}$ is only $4 \%$ larger than $\alpha_{N B}$ for a $200 \mathrm{~Pa}$ yield stress, but 23\% larger at 1,000 Pa. However, because $\alpha_{C}$ is always larger than $\alpha_{\mathrm{NB}}$, use of the buoyancy ratio derived with the weak sediment assumption is conservative (i.e., gives a larger buoyancy ratio) for predicting the occurrence of BDGREs in a stronger sediment.

On the other hand, the buoyancy ratio calculated by Eq. (1.18) is increasingly nonconservative as the yield stress exceeds that of the five BDGRE tanks. The slope of the assumed linear yield stress profile is calibrated to the BDGRE tanks where it averages $130 \mathrm{~Pa} / \mathrm{m}$ for AN-103 and AW-101, accounting for the effects of the heel layer (Hedengren et al. 2000). In a hypothetical strong sediment, where the yield stress varies from zero to 1,000 Pa over a depth of $1.5 \mathrm{~m}$, the slope is $670 \mathrm{~Pa} / \mathrm{m}$, a factor of 5 greater than the calibrating BDGRE tanks. To account for this steep slope, the buoyancy ratio criterion for Eq. (1.18) should be corrected as follows:

$$
\mathrm{BR}<(130 \mathrm{~Pa} / \mathrm{m})\left\lfloor\frac{\mathrm{H}_{\mathrm{S}}}{\tau_{\max }}\right\rfloor_{\text {strong }}
$$

where $\mathrm{H}_{\mathrm{S}}$ and $\tau_{\max }$ are the sediment depth and maximum yield stress for the strong sediment.

For the hypothetical tank with strong sediment above, the buoyancy ratio criterion prescribed by Eq. (5.6) would be $1 / 5=0.20$. For a uniform yield stress, the corrected buoyancy ratio limit 
is given by Eq. (5.4). While these criteria for preventing BDGREs maintain the calibration basis of the buoyancy ratio, they are extremely restrictive, and it is more practical to apply the flammability model instead.

Notwithstanding these corrections, application of the buoyancy ratio model to strong sediments is questionable in the first place. The buoyancy ratio model was derived under the assumption that the slow bubble migration responsible for background gas release can be represented by small, approximately spherical bubbles rising in accordance with the proportionalities of Stokes flow (Eq. 1.13). The exact bubble migration mechanism is unknown, and Stokes equation strictly applies only to Newtonian fluids. But we do know that sediments with the range of properties represented by the six BDGRE tanks retain gas as small, round (though not exactly spherical) bubbles. We also find that applying Stokes equation with the linearly increasing yield stress versus depth measured by the ball rheometer produces a parabolic gas volume fraction profile that is consistent with in situ retained gas measurements in these tanks. An approximately round bubble configuration is required to apply the buoyancy ratio model in accordance with its physical basis, and the predictions of the model are consistent with data for wastes in which approximately round bubbles exist.

These conditions have been relatively well defined by past gas retention research. A bubble maintains an approximately spherical shape if surface tension forces are larger than the forces due to the strength of the surrounding material. The diameter, $\mathrm{d}_{\mathrm{B}}$, of the largest round bubble for a given surface tension, $\sigma$, and material yield stress can be estimated by (Gauglitz et al. 1996)

$$
\mathrm{d}_{\mathrm{B}}=\frac{\sigma}{\tau_{\mathrm{y}}}
$$

The maximum bubble diameter of Eq. (5.7) is not an abrupt transition but a gradual distortion from spherical through ellipsoidal to an irregular dendritic shape. These elongated bubbles individually move through sediment in a series of abrupt jumps by a process called percolation, rather than the steady rise represented by Stokes flow (Stewart et al. 1996a). However, it is unclear whether this mechanism or another phenomenon represents the slow background migration of dendritic bubbles.

At the same time, a bubble that grows past the size at which it has sufficient buoyant force to rise through the surrounding material is no longer retained. This limiting bubble size also depends on the yield stress of the material via (Gauglitz et al. 1996)

$$
d_{B}=\frac{\tau_{y}}{\rho_{S} g_{G}}
$$

where $Y_{G}$ is a critical gravity-yield number estimated at 0.2 for incipient motion of a solid sphere in an unsheared viscoplastic medium (Stewart et al. 1996a). The bubble diameter criterion of 
Eq. (5.8) for round bubbles also applies to the maximum height of a dendritic bubble before percolation occurs.

The combination of the criteria expressed by Eq. (5.7) and (5.8) is illustrated in Figure 5.2 as a plot of bubble diameter versus yield stress. Above the solid diagonal line bubbles rise rapidly through the material and are not retained. Retained bubbles in the triangular area below both the dashed and solid lines are spherical to ellipsoidal while those above and to the right of the dash line are ellipsoidal to dendritic. The approximate bubble size and configuration of the BDGRE tanks, based on measured sediment properties and bubble sizes observed in core sample extrusions, plot as relatively small ellipsoidal bubbles. While the transition to a dendritic shape is not precise, the experimental observations of bubbles in both bentonite clay and waste samples show that a $200 \mathrm{~Pa}$ yield stress produces somewhat distorted ellipsoidal bubbles with diameters of a few millimeters, while those existing in clay or waste at $1,000 \mathrm{~Pa}$ are fully dendritic (Gauglitz et al. 1996). A reasonable estimate for the transition threshold is $500 \mathrm{~Pa}$.

The physical basis of the buoyancy ratio model would hold if dendritic bubbles in stronger sediments exhibit some kind of slow migration with functional dependencies similar to the Stokes flow-based mechanism assumed for round bubbles in weak sediment, and if it is at least as fast or faster. The detailed physics of dendritic bubble migration is not known, but some very indirect evidence that it is faster than in round bubbles might be inferred from early gas retention tests in clay simulants and actual waste samples. These tests showed that the maximum gas retention of about $40 \mathrm{vol} \%$ occurs in materials with a yield stress of around $100 \mathrm{~Pa}$ (Gauglitz et

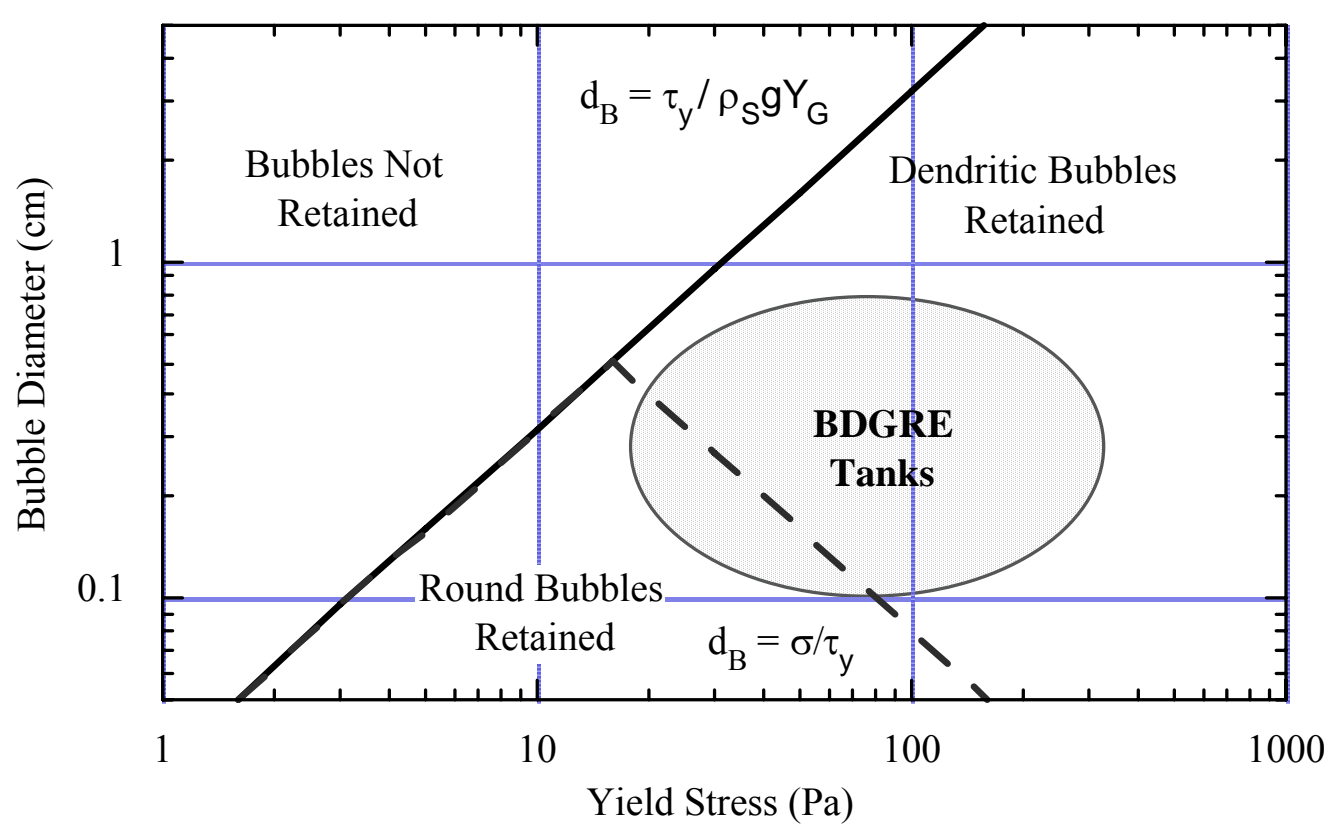

Figure 5.2. Bubble Shape and Retention Criteria Versus Yield Stress 
al. 1996). In stronger materials, the gas bubbles formed long, irregular dendritic channels that tended to stay open and allow gas to escape continuously, reducing the maximum gas retention to around $30 \mathrm{vol} \%$. (a)

Whether or not it has a sound physical basis, experience indicates that application of the buoyancy ratio to strong, even brittle, sediment may be conservative, or at least not nonconservative. Analysis of core sample extrusion video from AY-102 core samples taken approximately three months after it received the final hot sludge from C-106 indicates its sediment has a nearly uniform yield stress over 1,000 Pa (Onishi and Wells 2004).

The buoyancy ratio calculated via Eq. (1.18) for AY-102 from the median tank conditions and waste properties listed by Barker and Hedengren (2004) is 0.37. This strong sludge waste apparently has a uniform yield stress, so we apply the limiting buoyancy ratio from Eq. (5.4), which is 0.06. Even assuming a linearly increasing yield stress, the slope of the yield stress versus depth in AY-102 would be about six times that of the BDGRE tanks $(1000 \mathrm{~Pa} / 1.4 \mathrm{~m}=$ $710 \mathrm{~Pa} / \mathrm{m}$ versus $130 \mathrm{~Pa} / \mathrm{m}$ ), and the corresponding buoyancy ratio limit via Eq. (5.6) would be 0.17. Yet no obvious BDGREs have been observed in this tank, either in the waste level history or hydrogen monitoring through 2002 (three years after the transfer).

Though not conclusive, these results show that the predictions of the buoyancy ratio model with the limiting criterion defined by Eq. (5.4) for uniform yield stress or (5.6) for yield stress increasing linearly with depth are conservative, at least for AY-102. The preponderance of evidence listed in Table 5.1 supports its application to strong ductile sediments with these revised criteria. Strong sediments are defined as having a yield stress greater than $500 \mathrm{~Pa}$.

Table 5.1. Evidence for Applying Buoyancy Ratio to Strong Sediment

\begin{tabular}{||l|l||}
\hline \multicolumn{1}{|c|}{ Supporting Evidence } & \multicolumn{1}{c|}{ Opposing Evidence } \\
\hline $\begin{array}{l}\text { Faster migration of dendritic bubbles implied } \\
\text { by lower maximum gas retention in strong } \\
\text { simulant (Gauglitz et al. 1996) }\end{array}$ & $\begin{array}{l}\text { Bubbles are dendritic at } \tau_{\mathrm{y}}>\sim 500 \mathrm{~Pa}, \\
\text { migration mechanism and speed unknown } \\
\text { and likely different from round bubbles }\end{array}$ \\
\hline $\begin{array}{l}\text { BR increasingly conservative with } \tau_{\mathrm{y}} \text { because } \\
\alpha_{\mathrm{C}}>\alpha_{\mathrm{NB}} \text { (Eq. 5.5) }\end{array}$ & $\begin{array}{l}\text { BR non-conservative for constant } \tau_{\mathrm{y}} \text { slope } \\
\text { calibrated to BDGRE tanks [unless criterion } \\
\text { corrected per Eq. (5.4) or (5.6)] }\end{array}$ \\
\hline $\begin{array}{l}\text { Calculated BR demonstrated conservative for } \\
\text { strong sludge in AY-102 [with criteria } \\
\text { revised per Eq. (5.4) or (5.6)] }\end{array}$ & \\
\hline
\end{tabular}

(a) These tests were performed with no supernatant liquid so no buoyancy-induced release occurred. 


\subsubsection{Flammability Model in Strong Sediment}

As described in Section 5.2.3, strong waste is theoretically more likely to have uniform yield stress, which in weak sediments creates a linear gas volume fraction profile (Figure 5.1) that makes BDGREs small. If this also occurs for uniform yield stress in strong sediments, the predictions of the flammability model remain conservative. But there are no data to compare the size of BDGREs from strong sediments with the six historic BDGRE tanks, and Eq. (5.1), which shows that a uniform yield stress produces a linear gas volume fraction profile, requires the bubble migration mechanism to follow the Stokes flow analogy as assumed for weak waste.

While it is not possible to prove mathematically that the gas fraction increases with elevation with a uniform yield stress in strong sediment, there is some evidence that this is the case. The maximum vertical length of a dendritic bubble at percolation is uniform for a uniform yield stress via Eq. (5.8). This results in a uniform pressure difference across the bubble, which probably also implies at least a roughly uniform effective gas velocity and causes the gas volume fraction to increase with elevation. (a) Data from the detailed gas volume fraction measurements in AN-103 also help confirm that strong sediments have a uniform yield stress that produces a gas volume fraction that increases with elevation. This tank has a relatively deep heel layer of strong sediment with a relatively uniform yield stress of about 1,000 Pa (Gauglitz and Aiken 1997). As shown in Figure 5.3, the measured gas volume fraction in the heel layer appears to increase approximately linearly in the two risers (1B and 16B) in which measurements were taken. As the yield stress abruptly reverts to a linear decrease with elevation, the gas volume fraction also decreases roughly in accordance with Eq. (1.17). Additional discussion of heel layers in BDGRE tanks is given in subsection 5.2.7.2.

If the yield stress in strong sediment is not uniform but increases with depth like in the BDGRE tanks, and if the bubble migration mechanism is at least approximately the same in strong as in weak sediments, the same gas generation rate and sediment depth will produce a higher average gas volume fraction via Eq. (1.18). To conservatively account for this, the buoyancy ratio used to evaluate the flammability model must be adjusted by the inverse of Eq. (5.6) as follows:

$$
\mathrm{BR}_{\text {strong }}=\mathrm{BR}_{[\text {Eq.1.18] }} \frac{\left[\tau_{\max } / \mathrm{H}_{\mathrm{S}}\right]_{\text {strong }}}{(130 \mathrm{~Pa} / \mathrm{m})}
$$

If AY-102 had a yield stress increasing linearly with depth, for example, its buoyancy ratio for use in the flammability model would be $0.37 *(1000 / 1.4) / 130=2.0$.

(a) The gas velocity can be shown to increase with elevation and still produce an increasing gas volume fraction as long as the rate of velocity increase with elevation is slightly less than the specific volumetric gas generation rate. 


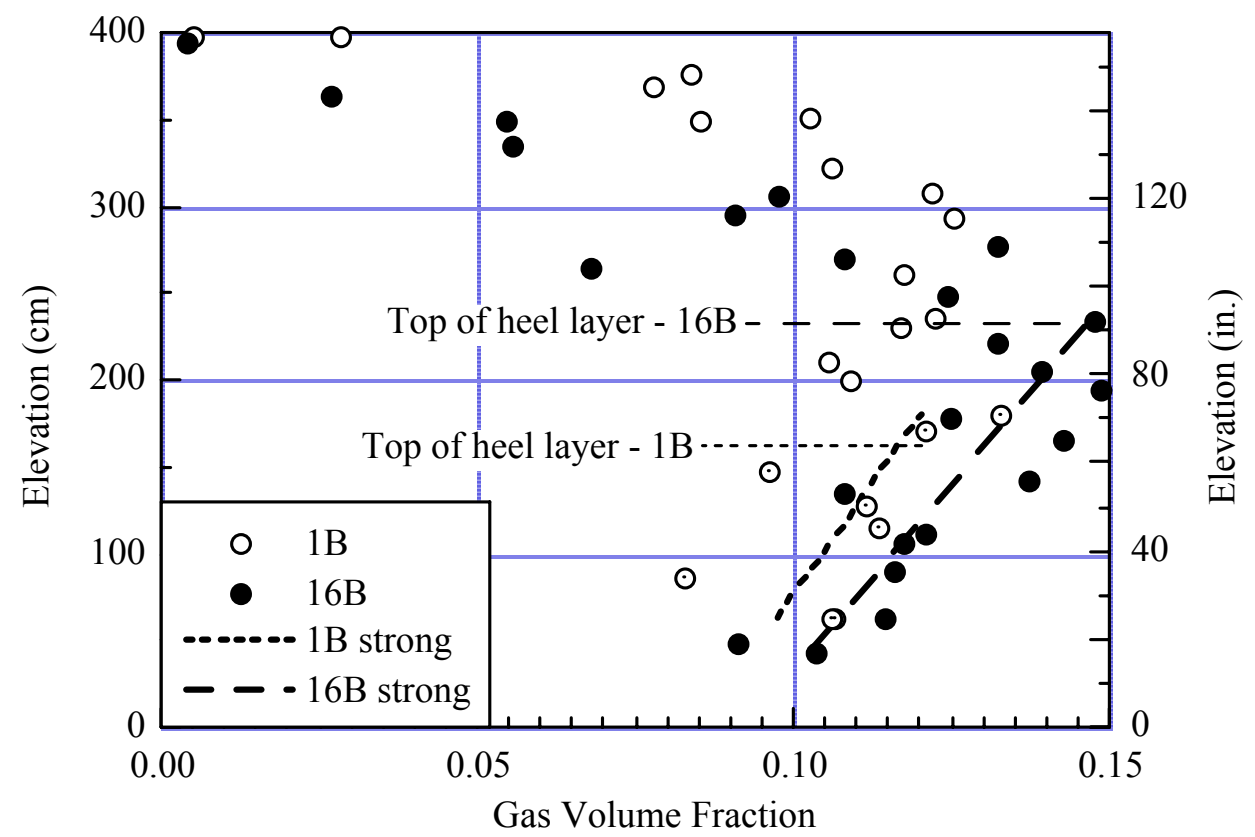

Figure 5.3. Gas Volume Fraction Profile in AN-103

There is some potentially opposing evidence for applying the flammability model to strong sediments, however. First, uniform yield stress, which apparently produces an increasing gas volume fraction with depth as it does in weak sediments, is not universal in strong sediments. A study of core sample extrusion video from T-110 and several 200-series tanks in B and T Farms by Rassat et al. (2003) shows that most have a yield stress in the range 500 to 2,000 Pa increasing approximately linearly with depth. One tank (B-204) had one core with a uniform yield stress and another in which it increased with depth. Although the waste in all these tanks is transuranic sludge, which may have different properties than other sludges or strong saltcake, it does show that a high yield stress is not automatically uniform.

Second, the most probable gob size based on instability analysis described in Appendix A is approximately proportional to the yield stress. Thus, stronger sediment would foster larger gobs and larger BDGREs. However, the analysis is a first-order perturbation to the classic solution for Rayleigh-Taylor instability in a liquid. The sediment is, in fact, assumed to become a liquid when motion due to the instability begins. Yield stress is an additional stress that affects the required density difference and gas fraction just prior to the instability. The gob size model does not apply in strong or brittle sediments that would not effectively act as liquid. This conclusion does not imply the effect is not present but that Appendix A cannot prove or quantify it.

Finally, because an increasing average yield stress increases the gas volume fraction necessary to initiate a BDGRE via Eq. (5.5), the gas release volume would be larger, at least for a yield stress increasing with sediment depth. However, this trend would be more than 
compensated for by the additional energy required to mobilize the gob and release the gas according to the energy ratio of Eq. (1.8).

In summary, while we have no data that describe BDGREs in strong sediments, the flammability model can be shown conservative for uniform yield stress or made conservative for nonuniform yield stress. If the yield stress is uniform in strong sediments, and this causes the gas volume fraction to increase with elevation, only very small BDGREs are possible. However, high yield stress is not necessarily uniform, so BDGREs are not necessarily limited in strong sediments, and the buoyancy ratio should be corrected by Eq. (5.9) if the yield stress is not known or known to increase with depth. The preponderance of evidence summarized in Table 5.2 supports the application of the flammability model for strong ductile sediments with this correction.

Table 5.2. Evidence for Applying the Flammability Model to Strong Sediment

\begin{tabular}{|l|l||}
\hline \multicolumn{1}{|c|}{ Supporting or Facilitating Evidence } & \multicolumn{1}{c|}{ Opposing or Constraining Evidence } \\
\hline $\begin{array}{l}\text { Uniform yield stress theoretically likely in } \\
\text { strong sediments which limits BDGRE size } \\
(\text { Section } 5.2 .3) .\end{array}$ & $\begin{array}{l}\text { Data show } \tau_{\mathrm{y}} \text { increases with depth in strong } \\
\text { sludge from some 200-series tanks, non- } \\
\text { conservative unless corrected by Eq. (5.9). }\end{array}$ \\
\hline $\begin{array}{l}\text { AN-103 data shows heel layer with uniform } \\
\tau_{\mathrm{y}} \sim 1,000 \text { Pa and linearly increasing } \alpha(\mathrm{z})\end{array}$ & $\begin{array}{l}\text { No BDGRE data to validate the empirical } \\
\text { model for strong sediment }\end{array}$ \\
\hline $\begin{array}{l}\text { Energy ratio decreases with } \tau_{\mathrm{y}} \text {, indicating a } \\
\text { lower fraction of gas released in a BDGRE }\end{array}$ & $\begin{array}{l}\text { BR somewhat nonconservative with } \tau_{\mathrm{y}} \\
\text { because } \alpha_{\mathrm{C}}>\alpha_{\mathrm{NB}}(\text { Eq. 5.5) causes larger } \\
\text { BDGRE }\left(\text { applies if } \tau_{\mathrm{y}} \text { increases with depth) }\right.\end{array}$ \\
\hline $\begin{array}{l}\text { Maximum dendritic bubble height (Eq. 5.8) is } \\
\text { uniform for uniform } \tau_{\mathrm{y}}, \text { implies increasing } \\
\alpha(\mathrm{z}) \text { with elevation, small BDGREs. }\end{array}$ & \multicolumn{2}{|l}{} \\
\hline
\end{tabular}

\subsubsection{Energy Ratio Model in Strong Sediment}

The yield stress is included explicitly in the denominator of the energy ratio expression of Eq. (1.8). Higher yield stress lowers the energy ratio in direct proportion and indicates a reduced gas release volume. For example, if the energy ratio were 10, indicating a rapid, efficient gas release in a waste with an average yield stress of $100 \mathrm{~Pa}$, it would drop to 2, indicating little or no gas release, if the yield stress were $500 \mathrm{~Pa}$. Beyond this the energy ratio is independent of scale, bubble size and shape limitations, or the yield stress profile, and it is not limited to any maximum yield stress.

\subsubsection{Brittle Sediment}

Though we think of strong sediment as being hard and brittle weak ones as soft and ductile, ductility really is not related directly to strength. When a force is applied to a ductile material it 
deforms elastically (returns to its original dimension when the force is released) until the stress reaches the elastic limit, and then plastically (experiences a permanent deformation) through much larger strains while the stress remains relatively high. A brittle material also deforms elastically up to a limiting stress but has a much smaller plastic strain before failure.

There have been no gas retention tests on brittle simulants and there are few or no data on gas retention or release in tanks that might have brittle sediments. In fact, the only way sediment can be determined to be brittle or ductile is to study core extrusion video tapes, which have been analyzed for only a few tanks. Thus it is difficult to justify the application of the BDGRE models to brittle sediments or to tanks with sediments of unknown properties that might be brittle, as explained below.

\subsubsection{Buoyancy Ratio and Flammability Model in Brittle Sediment}

There is no information available on the shape, size, or behavior of retained gas bubbles in a brittle material. There is no basis for conjecture about whether a uniform yield stress produces a gas volume fraction increasing with depth in a brittle sediment or whether the yield stress is likely to be uniform or not. Rassat et al (2003) shows a series of photographs of core extrusions from B-203 that indicates the sludge becomes both increasingly brittle and stronger with depth. On the other hand, the core extrusions from AY-102 show a uniform yield stress with different portions showing both brittle and ductile characteristics.

While brittle sediment clearly violates the fundamental assumptions of the buoyancy ratio model, and the data do not reveal a consistent behavior pattern in brittle sediments, there is the relatively strong evidence of conservatism of the buoyancy ratio for the uniform strength, partially brittle sediment in AY-102. This is the only item of evidence that supports application of the buoyancy ratio or flammability model to brittle sediments, and this would only apply to those with uniform yield stress.

\subsubsection{Energy Ratio Model in Brittle Sediment}

The fundamental assumption used to derive the energy ratio is that the sediment is ductile as explained in the following explanation of this assumption which is extracted from Stewart et al. (1996a). Salt slurries typical of DST waste behave differently than brittle, linear-elastic materials. Figure 5.4 shows stress-strain data for Bentonite clay simulant, with gas retention properties similar to sediments in the BDGRE tanks, at two different strain rates. (a) For strains less than about $10 \%$ the material is linear-elastic. As the stress increases above a certain critical value (about $190 \mathrm{~Pa}$ for the low strain-rate case and about 230 for the high strain-rate case), there is a sudden failure or relaxation. Beyond this point, a plastic region exists where the stress is approximately constant with strain. Finally, at a strain of about 1.6 for the low strain-rate case

(a) Phillips JR. "A Basic Survey of Simulant Materials for the Ball Rheometry Project." Internal letter report, Pacific Northwest National Laboratory, Richland, Washington. 

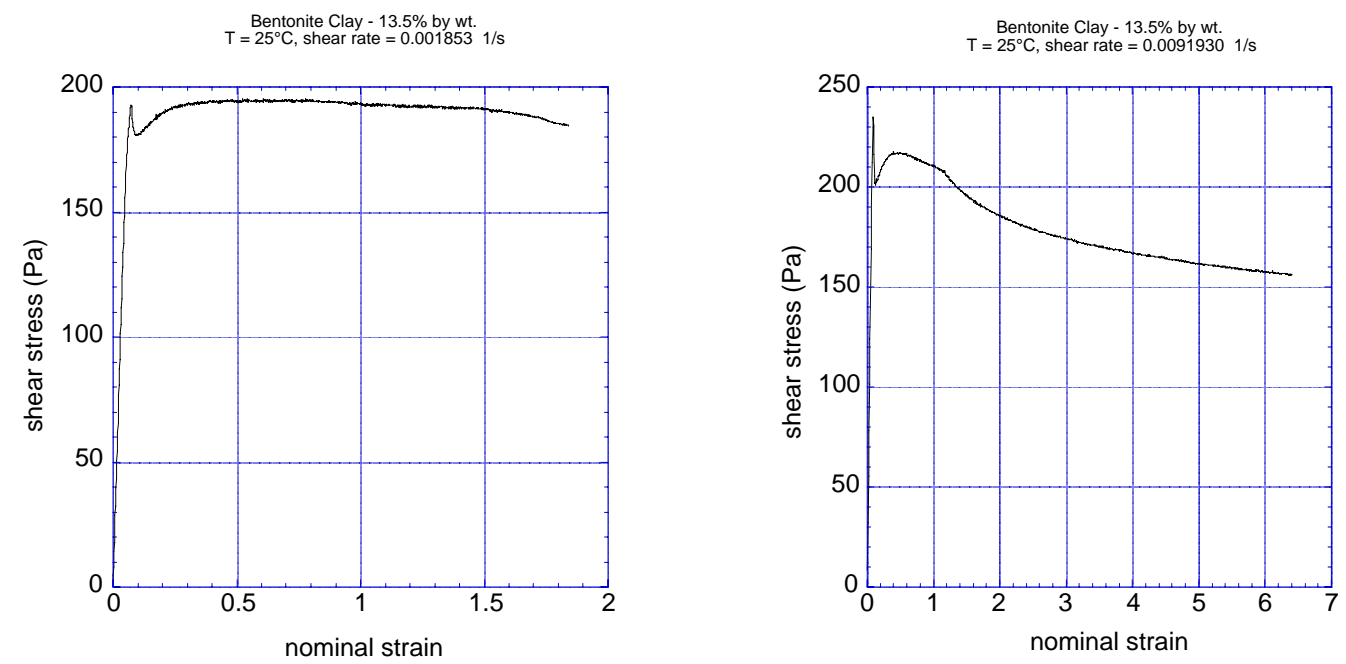

Figure 5.4. Stress-Strain Relationships for Bentonite Clay

and 1.2 for the high strain-rate case, a thixotropic region is encountered where the material is essentially flowing and no longer possesses solid-like properties.

The thixotropic region beginning at a nominal strain of approximately 1 is significant. At this strain, solid particles are displaced approximately one diameter. In doing this, they lose their memory of their initial state, so there is no longer a restoring force. With a small linear-elastic strain and a relatively constant stress in the plastic region, the energy required to yield the sediment is approximately $\mathrm{E}_{\mathrm{L}}=\varepsilon_{\mathrm{y}} \mathrm{V}_{\mathrm{s}} \tau_{\mathrm{y}}$, where $\varepsilon_{\mathrm{y}} \approx 1$ is the strain at thixotropic transition. With $V_{S}=\left(1-\alpha_{N B}\right) V_{\text {gob }}$, the required yield energy is expressed as Eq. (1.7).

In a more brittle material the stress would drop rapidly following the yield point and would not exhibit the wide, constant-stress plastic region. The energy input required to yield a brittle gob would be much less than given by Eq. (1.7) and the resulting value of energy ratio would be non-conservatively low. Therefore, the energy ratio does not provide useful information on the potential for gas release in brittle sediments. A large gas release should be assumed from BDGREs in brittle sediments unless the depth of supernatant liquid is truly minimal.

\subsubsection{Multiple Sediment Layers}

The new tanks being created by retrieval of single-shell tank wastes, evaporation of supernatant liquid, or transfer of waste from one double-shell tank to another will necessarily result in layers of sediment in the absence of mixing. It is probable that layered wastes will be come the rule in gas retention and release analysis. With some conservative adjustments on their inputs, application of the BDGRE models can be justified in most multiple-layer scenarios. 


\subsubsection{Buoyancy Ratio Model for Multiple Layers}

If weak saltcake sediment exists in two or more layers with somewhat different properties but that otherwise satisfy the primary assumptions of the model, the bubble migration process will apply in every layer, producing an approximately parabolic gas volume fraction profile with gas retention and release characteristics consistent with those of a single layer. The gas volume fraction profile in $\mathrm{AN}-103$ is a possible example (subsection 5.2.4.2). However, the inputs to the model should be modified to correctly accommodate the differences.

In the left frame of Figure 5.1, gas accumulation is greatest near the bottom of the sediment where the yield stress is highest and bubble migration velocity lowest. Therefore, the lower layer determines the average gas volume fraction, even if the upper layers have a higher gas generation rate. At the same time, the density of the upper layer describes the weight of the overburden and sets the requirement for buoyancy. This means that the buoyancy ratio, Eq. (1.18), should be evaluated with the gas generation rate of the lower layer and the sediment density from the upper layer. The sediment depth must equal the sum of the layers. However, if the upper layer makes up more than $50 \%$ of the sediment and has the higher gas generation rate, it should supply both the density and gas generation rate. In doubtful cases, using the maximum gas generation rate and minimum bulk density to represent the entire sediment layer in Eq. (1.18) ensures conservative results.

An extension of the layering logic above is where weak sediment is added on top of a hot relatively thin layer (less than $25 \%$ of the total sediment depth) with a very high gas generation rate. Buoyancy ratio predictions would be conservative in this case, even if the hot layer were very strong, even brittle. The hot layer provides a source of bubbles that must migrate through the weak sediment in addition to those generated there. (a) Therefore, as long as the entire layer is assumed to have the gas generation rate of the hot layer, and the hot layer makes up no more than about $25 \%$ of the total sediment, (b) buoyancy ratio predictions of BDGRE potential will be conservative.

\subsubsection{Flammability Model for Multiple Layers}

The flammability model will give conservative results for multiple layers of weak sediment, including the "hot" bottom layer described in above, provided that the buoyancy ratio used in the flammability model is evaluated with the maximum gas generation rate and the maximum sediment density (not the minimum as in the buoyancy ratio model). These choices create the maximum buoyancy ratio for evaluating the flammability model.

(a) Bubbles that might accumulate between the layers would soon become large enough to rise through the weak sediment without being retained in contrast to small bubbles that migrate directly into the weak layer.

(b) In Figure 5.1 , the bottom $25 \%$ of the sediment remains nonbuoyant and does not participate in a BDGRE. 
According to Section 5.2.4, the flammability model is conservative for layers of strong waste if the yield stress is uniform or the buoyancy ratio is adjusted via Eq. (5.9). This restriction does not apply to the hot bottom layer beneath a weak sediment or to a thin $(<25 \%$ of the total sediment depth) layer of strong waste on top of weak waste. In these cases, the buoyancy ratio can be calculated using the maximum gas generation rate and maximum density, as recommended above. Only a thin bottom layer, however, can be allowed to be brittle.

There are actually data to support application of the flammability model to these kinds of layered sediments (Hedengren et al. 2000). In addition to AN-103, as discussed in subsection 5.2.4.2, the bottom 15 to $60 \%$ of the sediment layer in several BDGRE tanks was much stronger $\left(\tau_{\mathrm{y}}>900 \mathrm{~Pa}\right)$ than the material above in measurements obtained in 1995-1996. The strong layer roughly corresponds to the depth of an initial heel left before final filling. Besides this heel, AN-104, SY-101 and SY-103 were filled in steps over a number of years, creating additional layers of sediment. In SY-103, the third and last layer (including the heel) consisted of sludge. In all cases the initial gas releases following the final fill were smaller than those that developed later, after the layers were mixed by the earlier BDGREs. (a) This history supports that application of the flammability model to layered sediments, as might follow a series of transfers, will yield conservative results.

\subsubsection{Energy Ratio Model for Multiple Layers}

The energy ratio model and criterion are based on uniform sediment properties. Like the buoyancy ratio and flammability models, the energy ratio can be conservatively applied to multiple layers provided the criterion is evaluated using the minimum yield stress and maximum neutral buoyancy gas volume fraction.

Because the energy ratio model is nonconservative for brittle sediments, the presence of brittle layers presents a problem. The central portion of the sediment carries most of the retained gas to be released and the energy ratio would be nonconservative if this region were brittle. However, if the brittle layer makes up the bottom or top $25 \%$ with ductile sediment in the central region, using the energy ratio is justified because the sediment with the highest gas volume will meet its requirements.

\subsubsection{Induced BDGREs}

The buoyancy ratio and flammability models provide information only about a long-term steady state or well-established cycle of spontaneous BDGREs. The models do not simulate the mechanisms at work during transient operations that might induce BDGREs such as decanting the supernatant liquid layer, starting a mixer pump, or otherwise globally disturbing the waste

(a) Because the postulated heel layer is probably not uniform and may participate in the larger BDGREs, the sediments in the six historic BDGRE tanks are treated as a single layer (see Section 5.3.1). 
and cannot be applied there. (a) BDGREs induced by decanting the liquid layer and mixing are analyzed in detail by Wells et al. (2002) and those induced by other globally waste disturbing activities by Stewart et al. (2003).

However, once a BDGRE begins, induced or spontaneous, the mechanisms expressed by the energy ratio still occur, and the energy ratio model and criteria remain applicable to assessing the gas release potential, provided it would have been applicable to the specific sediment in a spontaneous release.

\subsubsection{Liquid Added to Previously Dry Waste}

This limitation is already included in the methodology for assigning tanks to waste groups (Barker and Hedengren 2004). It was originally intended for single-shell tanks in which a deep layer of liquid was transferred into a tank with an existing layer of drained waste (i.e., with an interstitial liquid level below the waste surface level). Without the buoyant force of liquid surrounding them, the solid particles in this waste are compressed under a high lithostatic load. We believe this creates a hard, strong, brittle or granular waste with a very different character than assumed in deriving the buoyancy ratio model or observed in the BDGRE tanks. Adding liquid on top of this waste is not expected to make its properties similar to the BDGRE tanks on which the three models are based.

\subsubsection{Briefly Uncovered Sediment}

A normally submerged sediment layer may be uncovered briefly in a transfer operation before a supernatant liquid layer is re-established. As long as the interstitial liquid level remains approximately congruent with the average waste surface so the solids are not subject to a large, prolonged lithostatic load, the physical properties and gas retention characteristics of the waste are not be expected to change. If application of the buoyancy ratio, flammability model, or energy ratio were justified before uncovery, it should be justified afterward.

\subsubsection{Very Weak Sediment}

There is one gas release mechanism different from the buoyancy ratio but potentially producing similarly large gas releases. A cascading bubble disengagement gas release mechanism has been observed in small-scale experiments. In bentonite clay with yield stress of $10 \mathrm{~Pa}$, Gauglitz et al. (1996) observed a case where a single bubble became large enough (several millimeters) to break free and rise through the simulant. This small disturbance appeared to cause a cascade that eventually released essentially all bubbles present. The speed of the release

(a) Because the buoyancy ratio implies a specific gas volume fraction profile, it was used by Stewart et al. (2003) to estimate the initial quantity of retained gas in studying releases induced by global waste disturbances. This is not a prediction of whether induced BDGREs will occur. 
was similar to a buoyant displacement, and the release fraction was essentially $100 \%$. This kind of release was observed in simulants with yield stress up to $67 \mathrm{~Pa}$. The absence of this release behavior in stronger simulants $(200 \mathrm{~Pa})$ was postulated to be caused by continuous slow gas release from surface cracks which self-healed in the weaker simulant. However, these tests were conducted without a supernatant liquid layer and, except for the weakest simulant, the gas volume fractions at release were higher than neutral buoyancy and a BDGRE would have occurred before the bubble disengagement.

In sediment with a lower yield stress than the five BDGRE tanks, the buoyancy ratio with Eq. (1.18) is conservative except for the possibility of a bubble disengagement gas release when the average yield stress is less than $10 \mathrm{~Pa}$. However, possibly excepting a colloidal gel formed from phosphate precipitation, no tank waste is expected to have yield stress this low. Also, the experiments in which the bubble disengagement release was observed necessarily had an extremely high and uniform gas generation rate compared to a waste tank. With typical slow, nonuniform volumetric gas generation rates in the tanks, it would be very unlikely that a large group of bubbles would reach the size required to disengage from the sediment at the same time. Nevertheless, there are insufficient data to eliminate the possibility of a massive bubble disengagement in very weak sediments (yield stress $<\sim 10 \mathrm{~Pa}$ ).

\subsubsection{Changing Waste Temperatures}

The waste layer temperatures and temperature profiles decrease slowly year by year as radioactivity decays, reducing the heat load in the waste, or change somewhat more rapidly with adjusted annulus or headspace ventilation rates. (a) The primary case in point is the increase in annulus and headspace ventilation in AN-103, AN-104, AN-105, and AW-101 in mid-1996 that began a significant cooling trend that reached an approximate steady state about 1999-2000. The gas releases from these tanks generally became much smaller beginning in 1997. Some tanks had few or no releases at all until 1999. The decrease in gas generation rate from the resulting cooling reduces the calculated buoyancy ratio slightly, but not as much as the smaller gas releases seem to require. The buoyancy ratio for AW-101 actually increased slightly because of an increase in sediment depth. It may be that these cold tanks have not reached their final steady gas retention-release state where BDGREs will be larger, or that the subtle shift in the temperature profiles changed the gas retention behavior by some unknown mechanism. New in situ measurements could help confirm and define this possibility. In any case, the change in gas release volume predicted by the change in buoyancy ratio has so far shown to be conservative with respect to a temperature decrease.

The existing database biases the model toward a hot condition, and its predictions would likely be more consistent with a temperature increase. However, there are no data to define the effect of a reduction in ventilation, for example, increasing the waste temperature and

(a) The thermal inertia of the waste means that time constants for temperature changes are on the order of years. 
exacerbating BDGREs. Thus there is no basis for applying the flammability model to predict the result of increasing sediment temperatures. It is not clear to what extent reversing the temperature decrease in the four cold tanks would also reverse the reduction in BDGRE size. This limitation does not apply to new tanks with initially high-temperature waste. As summarized in Section 5.2.7, the experience of the historic BDGRE tanks is that the gas releases start small and increase in size as the waste cools and becomes more homogeneous.

\subsubsection{Small-Diameter Tanks}

Application of the buoyancy ratio-based criterion for flammability is predicated on the relationship of headspace to gob size defined by the six historic BDGRE tanks. The size of the unstable gob depends on the diameter and depth of the sediment, and the peak hydrogen concentration depends on the headspace volume relative to the release. It would be highly unlikely for the relationship of gob size to headspace in a small tank to be similar to the 100series waste tanks. There is no basis for using the buoyancy ratio-based model to predict peak hydrogen concentrations in any tank except those it is based upon.

This limitation does not apply to using the buoyancy ratio as an indicator of potential BDGRE behavior. The buoyancy ratio model is one-dimensional and theoretically does not depend on gob size, headspace, or tank aspect ratio. If the buoyancy ratio can be applied to sediment in a large tank, it can also be applied to the same sediment in a small tank. The energy ratio has been demonstrated independent of scale and should be valid in tanks of any size, as long as the sediment is not brittle.

\subsection{Determining Model Applicability from Waste Data}

The discussions and analyses above are summarized in Table 5.3. Each of the three models is listed: the buoyancy ratio for identifying BDGRE tanks (BR $<1)$, the buoyancy ratio-based flammability model for the limiting peak hydrogen concentration $\left\{\mathrm{BR}<\mathrm{f}\left(\mathrm{V}_{\mathrm{HS}},\left[\mathrm{H}_{2}\right]_{\text {limit }}\right)\right\}$, and the energy ratio $(\mathrm{ER}<3)$. For each sediment condition the basis for the application of the models is described as follows:

- $\mathrm{T}$ : supported by theory - application is in accordance with all the assumptions used in deriving the model.

- D: supported by data - application within range of experimental or tank data used to develop the model.

- P: supported by the preponderance of evidence described in tables in the appropriate section.

- C+, C-: conservative or nonconservative with respect to flammable gas hazard with conservatism or nonconservatism mathematically quantified or demonstrated by data or logical argument. 
Table 5.3. Basis for Applying Buoyancy Ratio, Flammability and Energy Ratio Models ${ }^{(a)}$

\begin{tabular}{|c|c|c|c|c|}
\hline Sediment Condition & BR $<1$ & \begin{tabular}{|c|}
$\mathbf{B R}<\mathbf{f}\left(\mathbf{V}_{\mathrm{HS}}\right.$ \\
$\left.\left[\mathbf{H}_{2}\right]_{\text {limit }}\right)$ \\
\end{tabular} & $\mathbf{E R}<\mathbf{3}$ & Comment \\
\hline $\begin{array}{l}\text { Weak, ductile, linearly increasing } \\
\tau_{\mathrm{y}} \text { vs. z }\end{array}$ & $\mathrm{T}, \mathrm{D}$ & $\mathrm{D}$ & $\mathrm{T}$ & $\begin{array}{l}\text { Basic assumption for models, data } \\
\text { from historic BDGRE tanks }\end{array}$ \\
\hline Deep sediment (not brittle) & $\mathrm{T}, \mathrm{D}$ & $\mathrm{D}$ & $\mathrm{T}$ & $\begin{array}{l}\text { Data from SY-101 justifies } 7 \mathrm{~m} \\
\text { depth (Sec. 5.2.2) }\end{array}$ \\
\hline Weak, ductile, uniform $\tau_{\mathrm{y}}$ vs. $\mathrm{z}$ & $\mathrm{T}^{(\mathrm{b})}$ & $\mathrm{C}+$ & $\mathrm{T}, \mathrm{D}$ & $\begin{array}{l}\text { Bubble migration mechanism } \\
\left.\text { same as linear } \tau_{\mathrm{y}} \text { vs. } \mathrm{z} \text { (Sec. } 5.2 .3\right)\end{array}$ \\
\hline $\begin{array}{l}\text { Strong, ductile, linearly increasing } \\
\tau_{\mathrm{y}} \text { vs. } \mathrm{z}\end{array}$ & $\mathrm{T}^{(\mathrm{c})}$ & $\mathrm{P}^{(\mathrm{d})}$ & $\mathrm{T}$ & Sec. 5.2.4 \\
\hline Strong, ductile, uniform $\tau_{\mathrm{y}}$ vs. $\mathrm{z}$ & $\mathrm{T}^{(\mathrm{b})}$ & $\mathrm{P}$ & $\mathrm{T}$ & Sec. 5.2 .4 \\
\hline Brittle (weak or strong) & $-{ }^{(e)}$ & - & $\mathrm{C}-$ & Sec. 5.2 .5 \\
\hline $\begin{array}{l}\text { Multiple layers, including hot } \\
\text { bottom layer }\end{array}$ & $\mathrm{C}+{ }^{(\mathrm{f})}$ & $\mathrm{C}+{ }^{(\mathrm{f})}$ & $\mathrm{T}$ & Bottom layer $<25 \% \mathrm{H}_{\mathrm{S}}(\mathrm{Sec} .5 .2 .6)$ \\
\hline Induced BDGREs & - & - & $\mathrm{T}$ & Sec. 5.2.7 \\
\hline Liquid added to dry waste & - & - & - & Sec. 5.2.8 \\
\hline Briefly uncovered sediment & $\mathrm{T}$ & $\mathrm{T}$ & $\mathrm{T}$ & $\begin{array}{l}\text { Brief uncovery doesn't change } \\
\text { properties of sediment (Sec. 5.2.9) }\end{array}$ \\
\hline Very weak sediment & - & - & - & $\begin{array}{l}\text { Criteria for bubble disengagement } \\
\text { unknown (Sec. 5.2.10) }\end{array}$ \\
\hline Cooling & $\mathrm{T}, \mathrm{D}$ & $\mathrm{D}, \mathrm{C}+$ & $\mathrm{T}$ & Data are cold tanks (Sec. 5.2.11) \\
\hline Heating & $\mathrm{T}$ & $-(\mathrm{g})$ & $\mathrm{T}$ & Sec. 5.2.11. \\
\hline Small-diameter tanks & $\mathrm{T}$ & - & $\mathrm{T}$ & Sec. 5.2.12. \\
\hline & \multicolumn{4}{|c|}{$\begin{array}{l}\text { D: supported by data P: supported by preponderance of evidence } \\
\text { C-: shown non-conservative }-: \text { no entry }\end{array}$} \\
\hline \multicolumn{5}{|c|}{$\begin{array}{l}\text { Notes: } \\
\text { (a) Gray shading indicates conditions where application of the models is not supp } \\
\text { the preponderance of evidence, or specifically shown conservative } \\
\text { (b) BR criterion adjusted by Eq. (5.4). } \\
\text { (c) BR criterion adjusted Eq. (5.6). } \\
\text { (d) BR adjusted by Eq. (5.9) } \\
\text { (e) Observations show that the model bounds the BDGRE behavior in AY-102. } \\
\text { (f) Using conservative calculation inputs specified in Section 5.2.6. } \\
\text { (g) Does not imply a limitation on initially high-temperature sediment. }\end{array}$} \\
\hline
\end{tabular}

\subsubsection{Brittle Sediment Issue}

Brittle sediment violates the basic assumptions of the buoyancy ratio and energy ratio models and is outside the range of the flammability model represented by the six historic BDGRE tanks. Strong ductile sediments (yield stress $>500 \mathrm{~Pa}$ ) are also outside the bases of the buoyancy ratio and flammability models, but the preponderance of evidence supports their application with some adjustments (Section 5.2.4). In either case there are few data available to determine sediment rheology, and what information is available indicates many tanks may contain strong and/or brittle sediments. Scanning electron microscopy (SEM) of AY-102 sludge samples revealed large agglomerates of manganese and iron oxy-hydroxide. It may be that aluminum- 
and chromium-rich waste sludge would tend to be ductile while iron- and manganese-rich sludge would tend to be brittle, but this has not yet been demonstrated.(a)

In addition to this possibility, there are no reliable rheology data for most tanks and no proven methodology to predict the rheology for new sediments (e.g., those resulting from transfers, concentration). Only in situ measurements (e.g., with the ball rheometer) can correctly capture the combined effects of the lithostatic load and interparticle attraction on the sediment rheology at the actual waste temperature (Barker et al. 1999). The only in situ data available are from the ball rheometer deployments in the five current BDGRE tanks (Stewart et al. 1996b).(b) Yield stress estimates based on core sample extrusions (Gauglitz and Aiken 1997, Rassat et al. 2003, Onishi and Wells 2004) appear to provide values similar to the ball rheometer on the average, at least for tanks with relatively low waste temperatures, where solids precipitation is not likely when the sample is cooled. Core extrusions provide the only available indication of brittleness versus ductility. Yield stresses from ex-tank rheometer measurements on waste samples do not compare well consistently with ball rheometer or core sample extrusion estimates except possibly in sludge waste.(c)

In the past we tacitly assumed that all the saltcake and sludge sediments in double-shell tanks, except possibly the very hot, shallow sediments in tanks like AZ-101, were weak and behaved like those in the six BDGRE tanks. The reasoning was that, because they all formed from settled solids of similar particle size and existed under deep layers of liquid, they should have similar properties. Additionally, gas retention was apparently similar in sludge and saltcake (Barker et al. 1999). However, some recent data imply that the weak waste in the six BDGRE tanks may be the exception, and most waste, particularly sludge, may be stronger and more brittle. Core extrusions from AY-102, one of the tanks that we claim to validate the predictions of the buoyancy ratio model, indicate uniform yield stress of about 1,100 $\mathrm{Pa}$, and portions of the material may also be brittle (Onishi and Wells 2004). Core extrusions from B-203 indicate TRU sludge becomes both increasingly brittle and stronger with depth (Rassat et al. 2003). Photos of SY-103 core extrusions from 1986, before the last waste addition in 1988 when BDGREs began, indicate a very stiff, possibly brittle sludge (Stewart et al. 1996b). However, extrusions from 1994 indicate a weak, ductile material to within $2 \mathrm{ft}$ of the tank bottom.

At the same time, the in situ rheology measurements in the six BDGRE tanks show they also contain strong, but probably not brittle, heel layers (Sections 5.2.4 and 5.2.7). In eight of the ten ball rheometer deployments in five of these tanks (one run in each of two risers) the ball stopped above the tank bottom, indicating a yield stress exceeding $900 \mathrm{~Pa}$ (the strength calculated

(a) Personal communication with G Cooke, CH2M HILL interoffice memo 7S110-DLH-03-007, from Analytical Process Development to KG Carothers, October 17, 2003. "Caustic Demand Test Results, Tank 241-AY-102 Sludge."

(b) The ball rheometer was also run in SY-101 two years after the mixer pump was installed. Thus these data give no information directly related to BDGREs in this tank.

(c) Wells BE and SA Barker. 2003. Summary of Yield Stress in Shear Data for Hanford Waste. Letter report TWS03.044, Pacific Northwest National Laboratory, Richland, Washington (Appendix F in Barker and Hedengren 2004). 
necessary to support the net weight of the ball). The elevation at which the ball stopped in these eight cases averaged $30 \%$ of the sediment depth and ranged from 15 to $60 \%$ of the depth, roughly corresponding to the depth of the waste heel left in the tank prior to the final fill. However, this stronger layer is quite nonuniform and may sometimes participate in BDGREs, as evidenced by changes in sediment temperature profiles. Current in situ rheology measurements would show whether these strong layers have remained stable or have grown as the sediment temperatures decreased.

The property that makes a material brittle is the ease of crack propagation. In a ductile material, the tip of a crack tends to "blunt out" and form a void instead of continuing to propagate. In a brittle material, the crack continues to propagate, eventually becoming a fracture. (a) Gas retention in ceramic nuclear fuel pellets in commercial power reactors appears consistent with this behavior. Gas buildup has been observed to cause general fracturing of fuel pellets, allowing gas to pressurize the fuel tube cladding. If brittle waste storage tanks behave this way, gas produced by radiolysis and thermolysis would eventually form cracks that would eventually allow the gas to escape continuously, preventing BDGREs altogether. On the other hand, no evidence of pre-existing cracks is visible in core sample extrusions that we would classify as brittle. However, even brittle sludge samples show a high liquid fraction compared with a dry ceramic fuel pellet. The liquid content might allow cracks to heal under a large applied strain such as during core sampling. A similar healing mechanism might close cracks formed by gas accumulation. This area needs much more study before any meaningful conclusions are possible.

\subsubsection{BDGRE Database Issue}

While it is fortunate that BDGRE data come from a very small number of tanks and that number is growing smaller rather than larger, an empirical model like the flammability model of Section 4.2 is severely limited by the narrow database. However, the database representing the current BDGRE tanks can and should be improved. The fundamental need is to be able to relate tank behavior to the tank's waste configuration and properties. This requires both gas monitoring data to describe the BDGRE behavior and waste data from the same time period to describe tank conditions.

Fortuitously, we have more than just six tanks to analyze. There are two demonstrably different waste conditions in four cold tanks coupled with a BDGRE behavior much different from their prior hot state. We also have one hot tank (SY-103) that did not change either

conditions or behavior (as far as we know) and one tank (SY-101) that actually defined the conditions required for BDGREs large enough to create a flammable headspace. ${ }^{(b)}$

(a) Personal communication with Dr. Fred Simonen, PNNL Laboratory Fellow in structural mechanics.

(b) No BDGREs have occurred in SY-101 since mixer pump operation began in 1993. The potential for BDGREs in SY-101 was removed by waste transfer and dilution in 2000 . 
The data describe the conditions and BDGRE behavior in the hot tanks relatively well, but the data for the cold tanks is less adequate. Except for the sediment temperature, waste level, and, to some extent, the sediment depth, cold tank conditions and waste properties are assumed to be the same as their previous hot state. This assumption is highly suspect for the sediment density and yield stress, but no data are available to replace the assumption. (a) Likewise, the BDGRE record for the cold tanks based on gas monitoring data from 1996 through September 2002 spans what might be a transitional period leading up to a "mature" behavior that might have been revealed by more recent data if gas monitoring systems had not been shut down (see Section 3.2.7).

(a) Densities derived from recent core samples are inconsistent with the temperature trend and previous density measurements, as discussed in Section 3.2.2. 


\subsection{Conclusion and Recommendations on Model Applicability}

A coherent body of knowledge and theory to explain the mechanisms relating gas release behavior to tank conditions exist only for the six historic BDGRE tanks, and adequate data on gas release behavior, waste configuration, and properties are available to represent only the period from 1994 to 1996 (SY-101 from 1989 to 1993). The buoyancy ratio and flammability models represent only these tanks and others that can be shown to be very much like them, though application to a wider range of conditions can be justified by theory, data, or the preponderance of evidence. Such conditions include sediments with uniform yield stress, strong ductile sediments, and multiple layers. However, there is no basis for applying the BDGRE models to tanks with brittle sediments, very weak sediments, or cases where liquid is added to previously dry waste, among others.

Because brittle sediments are outside the models' basis, the yield stress profile is an important consideration, and adjustments need to be applied to calculations involving strong sediments to ensure conservatism, it is important to determine which tanks contain strong or brittle sediments and how these properties vary with depth. It is particularly important to confirm whether sludges are generally brittle or not. A rough estimation of whether an existing tank has weak, strong, or brittle sediment, and whether or not the yield stress is uniform, can be made relatively quickly and easily by simply completing the review of available video tapes of core sample extrusions. However, only in situ rheology measurements can confirm the extrusion results and reveal the yield stress profile.

Also, in the absence of current data on tank behavior and waste conditions, the knowledge base on the BDGRE tanks is on the verge of becoming obsolete. To confirm whether or describe how their BDGRE behavior has changed, some manner of continuous gas monitoring (at least hydrogen) should be re-established on the current BDGRE tanks and a few borderline tanks like AY-102 and AN-107. Waste descriptions should be updated by in situ rheology and density measurements. This should include SY-101 as well other tanks with gas monitoring restored.

Finally, the demands of storing more and more waste in the same number of DSTs will necessarily create waste configurations different from any we have measured or analyzed in the past. Therefore, a long-term program for waste data collection, management, and analysis should be initiated to maintain the technical basis for flammable gas safety evaluation as conditions evolve. 


\subsection{References}

Allemann RT, ZI Antoniak, WD Chvala, LE Efferding, JG Fadeff, JR Friley, WB Gregory, JD Hudson, JJ Irwin, NW Kirch, TE Michener, FE Panisko, CW Stewart, and BM Wise. 1994. Mitigation of Tank 241-SY-101 by Pump Mixing: Results of Testing Phases A and B. PNL-9423, Pacific Northwest Laboratory, Richland, Washington.

Antoniak ZI. 1993. Historical Trends in Tank 241-SY-101 Waste Temperatures and Levels. PNL-8880, Pacific Northwest Laboratory, Richland, Washington.

Babad H, GD Johnson, JA Lechelt, DA Reynolds, LR Pederson, DM Strachan, D Meisel, C Jonah, and EC Ashby. 1991. Evaluation of the Generation and Release of Flammable Gases in Tank 241-SY-101. WHC-EP-0517, Westinghouse Hanford Company, Richland, Washington.

Barker SA. 2003. Tank Waste Volume Calculator. RPP-13019, CH2M HILL Hanford Group, Richland, Washington.

Barker SA, and DC Hedengren. 2003. Methodology and Calculations for the Assignment of Waste Groups for the Large Underground Waste Storage Tanks at the Hanford Site. RPP-10006 Rev. 0, CH2M HILL Hanford Group, Richland, Washington.

Barker SA and DC Hedengren. 2004. Methodology and Calculations for the Assignment of Waste Groups for the Large Underground Waste Storage Tanks at the Hanford Site. RPP-10006 Rev. 4, CH2M HILL Hanford Group, Richland, Washington.

Barker SA, WB Barton, DR Bratzel, M Epstein, PA Gauglitz, GD Johnson, SN Maruvada, CE Olson, ML Sauer, SE Slezak, CW Stewart, and J Young. 1999. Flammable Gas Safety Analysis Review. SNL-000198, Sandia National Laboratory, Albuquerque, New Mexico.

Bohl WR. 1997. Remarks on Void Development in Hanford Double-Shell-Tank Sludge. LA-UR-97-3823, Los Alamos National Laboratory, Los Alamos, New Mexico.

Brewster ME, NB Gallagher, JD Hudson, and CW Stewart. 1995. The Behavior, Quantity, and Location of Undissolved Gas in Tank 241-SY-101. PNL-10681, Pacific Northwest Laboratory, Richland, Washington.

Estey SD and MD Guthrie. 1996. An Analysis of Parameters Describing Gas Retention and Release Behavior in Double-Shell Tank Waste. WHC-SD-WM-TI-755, Westinghouse Hanford Company, Richland, Washington.

Fan LS and K Tsuchiya. 1990. Bubble Wake Dynamics in Liquids and Liquid-Solid Suspensions. Butterworth Heinemann, Stoneham, Massachusetts.

Fowler KD. 1995. Tank Farm Waste Compatibility Program. WHC-SD-WM-OSD-015 Rev. 1, Westinghouse Hanford Company, Richland, Washington. 
Gauglitz PA, SD Rassat, PR Bredt, JH Konynenbelt, JM Tingey, and DP Mendoza. 1996. Mechanisms of Gas Bubble Retention and Release: Results for Hanford Waste Tanks 241-S-102 and 241-SY-103 and Single-Shell Tank Simulants. PNNL-11298, Pacific Northwest National Laboratory, Richland, Washington.

Gauglitz PA and JT Aikin. 1997. Waste Behavior During Horizontal Extrusion: Effect of Waste Strength for Bentonite and Kaolin/Ludox Simulants and Strength Estimates for Wastes from Hanford Waste Tanks 241-SY-103, AW-101, AN-103 and S-102. PNNL-11706, Pacific Northwest National Laboratory, Richland, Washington.

Hedengren DC, KM Hodgson, WB Barton, CW Stewart, JM Cuta, and BE Wells. 2000. Data and Observations on Double-Shell Flammable Gas Watch List Tank Behavior. RPP-6655 Rev. 0, CH2M HILL Hanford Group, Richland, Washington.

Hedengren DC, TA Hu, MA Kufahl, DJ McCain, CW Stewart, JL Huckaby, LA Mahoney, and KG Rappe. 2001. Data and Observations of Single-Shell Flammable Gas Watch List Tank Behavior. RPP-7249 Rev. 0, CH2M HILL Hanford Group, Richland, Washington.

Hedengren DC. 2003. Flammable Gas Monitoring Results in Single-Shell Tank and DoubleShell Tank Vapor Spaces for the Period of July 1, 2001 Through September 30, 2002. RPP15997, CH2M HILL Hanford Group, Richland, Washington.

Herting DL. 1997. Results of Dilution Studies with Waste from Tank 241-AN-105. HNF-SD-WM-DTR-046 Rev. 0, Fluor Daniel Hanford, Inc., Richland, Washington.

Herting DL. 1998. Results of Dilution Studies with Waste from Tank 241-AN-104. HNF-3352 Rev. 0, Fluor Daniel Hanford, Inc., Richland, Washington.

Herting DL, JF O'Rourke, DW Edmonson, JW Chenault, and JC Person. 1999. Results of Dilution Studies with Waste from Tank-AW-101. HNF-4964 Rev. 0, Fluor Daniel Hanford, Inc., Richland, Washington.

Hodgson KM, RP Anantatmula, SA Barker, KD Fowler, JD Hopkins, JA Lechelt, DA Reynolds, DC Hedengren, RE Stout, and RT Winward. 1996. Evaluation of Hanford Tanks for Trapped Gas. WHC-SD-WM-ER-526 Rev. 1, Westinghouse Hanford Company, Richland, Washington.

Hopkins JD. 1995. Methodology for Flammable Gas Evaluations. WHC-SD-WM-TI-724, Westinghouse Hanford Company, Richland, Washington.

Hu TA. 2004. Empirical Rate Equation Model and Rate Calculations of Hydrogen Generation for Hanford Tank Waste. RPP-3851 Rev. 1, CH2M HILL Hanford Group, Richland, Washington.

Hu TA. 2005. Steady State Flammable Gas Release Rate Calculation and Lower Flammability Level Evaluation for Hanford Tank Waste. RPP-5926 Rev. 4A, CH2M HILL Hanford Group, Inc, Richland, Washington. 
Johnson GD. 1996. Evaluation of Recommendation for Addition of Tanks to the Flammable Gas Watch List. WHC-SD-WM-ER-594 Rev. 0, Westinghouse Hanford Company, Richland, Washington.

Johnson GD, NW Kirch, RE Bauer, JM Conner, CW Stewart, BE Wells, and JM Grigsby. 2000. Evaluation of Hanford High-Level Waste Tank 241-SY-101. RPP-6517 Rev. 0, CH2M HILL Hanford Group, Richland, Washington.

Johnson GD, DC Hedengren, JM Grigsby, CW Stewart, JJ Zach and LM Stock. 2001. Flammable Gas Safety Issue Resolution. RPP-7771, CH2M HILL Hanford Group, Inc., Richland, Washington.

Kirch NW and JE Meacham. 2004. Flammable Gas Waste Group Assignment FY2004-ENG-S0133. RPP-21336, CH2M HILL Hanford Group, Inc., Richland, Washington.

Mahoney LA, ZI Antoniak, JM Bates, and ME Dahl. 1999. Retained Gas Sampling Results for the Flammable Gas Program. PNNL-13000, Pacific Northwest National Laboratory, Richland, Washington.

Mendenhall W, D Wackerly, and R Scheaffer. 1990. Mathematical Statistics with Applications. PWS-KENT Publishing Company, Boston.

Meyer PA, ME Brewster, SA Bryan, G Chen, LR Pederson, CW Stewart, and G Terrones. 1997. Gas Retention and Release Behavior in Hanford Double Shell Waste Tanks. PNNL-11536 Rev. 1, Pacific Northwest National Laboratory, Richland, Washington.

Meyer PA and BE Wells. February 2000. "Understanding Gas Release Events in Hanford Double-Shell Tanks." Proceedings of Waste Management 2000, Tucson, Arizona.

Onishi Y, BE Wells, SA Hartley, and SK Cooley. 2002. Pipeline Cross-Site Transfer Assessment for Tank 241-SY-101. PNNL-13650, Pacific Northwest National Laboratory, Richland, Washington.

Onishi Y and BE Wells. 2004. Feasibility Study on Using Two Mixer Pumps for Tank 241-AY102 Waste Mixing. PNNL-14763, Pacific Northwest National Laboratory, Richland, Washington.

Rassat SD, CW Stewart, BE Wells, JM Cuta, WL Kuhn, ZI Antoniak, KP Recknagle, G Terrones, JH Sukamto, VV Viswanathan, and DP Mendoza. 2000. Dynamics of Crust Dissolution and Gas Release in Tank 241-SY-101. PNNL-13112, Pacific Northwest National Laboratory, Richland, Washington.

Rassat SD LA Mahoney, BE Wells, DP Mendoza, and DD Caldwell. 2003. Assessment of Physical Properties of Transuranic Waste in Hanford Single-Shell Tanks. PNNL-14221, Pacific Northwest National Laboratory, Richland, Washington. 
Slezak SE, DC Williams, W Cheng, F Gelbard and DR Bratzel. 1998. Refined Safety Analysis Methodology for Flammable Gas Risk Assessment in the Hanford Site Tanks. HNF-SD-WMES-410 Rev. 2, Flour Daniel Hanford, Inc., Richland, Washington.

Stewart, CW, JD Hudson, JR Friley, FE Panisko, ZI Antoniak, JJ Irwin, JG Fadeff, LE Efferding, TE Michener, NW Kirch, and DA Reynolds. 1994. Mitigation of Tank 241-SY-101 by Pump Mixing: Results of Full-Scale Testing. PNL-9959, Pacific Northwest Laboratory, Richland, Washington.

Stewart CW, CL Shepard, JM Alzheimer, TI Stokes and G Terrones. 1995. In Situ Determination of Rheological Properties and Void Fraction in Hanford Waste Tank 214-SY-101. PNL-10682, Pacific Northwest Laboratory, Richland, Washington.

Stewart CW, ME Brewster, PA Gauglitz, LA Mahoney, PA Meyer, KP Recknagle, and HC Reid. 1996a. Gas Retention and Release Behavior in Hanford Single-Shell Waste Tanks. PNNL-11391, Pacific Northwest National Laboratory, Richland, Washington.

Stewart CW, JM Alzheimer, ME Brewster, G Chen, RE Mendoza, HC Reid, CL Shepard, and G Terrones. 1996b. In Situ Rheology and Gas Volume in Hanford Double-Shell Waste Tanks. PNNL-11296, Pacific Northwest National Laboratory, Richland, Washington.

Stewart CW, JL Huckaby, and PA Meyer. 2003. Effects of Globally Waste-Disturbing Activities on Gas Generation, Retention, and Release in Hanford Waste Tanks. PNNL-13781, Rev. 2, Pacific Northwest National Laboratory, Richland, Washington.

Stewart CW, Meyer PA, and JL Huckaby. 2003. Effects of Globally Waste Disturbing Activities on Gas Generation, Retention, and Release in Hanford Waste Tanks. PNNL-13781 Rev. 2, Pacific Northwest National Laboratory, Richland, Washington.

Stewart CW and PA Meyer. 2005. Derivation of the Buoyancy Ratio Equation from the Bubble Migration Model. Addendum to PNNL-13337, Preventing Buoyant Displacement Gas Release Events in Hanford Double-Shell Waste Tanks, Pacific Northwest National Laboratory, Richland, Washington.

Sullivan HL. 1995. A Safety Assessment for Proposed Pump Mixing Operations to Migrate Episodic Gas Releases in Tank 241-SY-101: Hanford Site, Richland, Washington. LA-UR-923196 Rev. 14, Los Alamos National Laboratory, Los Alamos, New Mexico.

Tingey JM, PR Bredt, and EH Shade. 1994. The Effects of Heating and Dilution on the Rheological and Physical Properties of Tank 241-SY-101 Waste. PNL-10198, Pacific Northwest National Laboratory, Richland, Washington.

Tran TT. 1993. Thermocouple Status Single-Shell and Double Shell Waste Tanks. WHC-SD-WM-TI-553 Rev. 0, Westinghouse Hanford Company, Richland, Washington. 
Van Vleet RJ. 1996. Summary of Flammable Gas Hazards and Potential Consequences in Tank Waste Remediation Facilities at the Hanford Site. WHC-SD-WM-TI-753 Rev. 0, Westinghouse Hanford Company, Richland, Washington.

Wells BE, JM Cuta, SA Hartley, LA Mahoney, PA Meyer and CW Stewart. 2002. Analysis of Induced Gas Releases During Retrieval of Hanford Double-Shell Tank Waste. PNNL-13782, Pacific Northwest National Laboratory, Richland, Washington.

Whitney PD. 1995. Screening the Hanford Tanks for Trapped Gas. PNL-10281, Pacific Northwest National Laboratory, Richland, Washington. 


\section{Appendix A}

Gas Release from Buoyant Sediment Layers 


\section{Appendix A - Gas Release from Buoyant Sediment Layers}

\section{A.1 Stability of Buoyant Sediment Layers}

At the instant a buoyant eruption begins, the yield strength of the sediment around the buoyant gob is exceeded and the material begins to flow. The buoyant gob begins to rise and liquid begins to flow around and down to replace it. We want to find a length scale that relates the size of the rising gob to the depth and physical properties of the liquid and sediment layers. Instability theory describes such a length scale for adjacent, semi-infinite planar layers of Newtonian fluids of different uniform densities and viscosities. When the density of the lower fluid is less than that of the overlaying one, parcels of the light fluid rise while equal volumes of the heavy fluid fall forming a wave at the interface between the two fluids. The length scale is half the wave length of the disturbance that has the largest growth rate. If the length scale remains approximately constant as the disturbance grows, then it defines the effective size of the parcels of fluid.

Because the sediment layer in waste tanks is non-Newtonian and finite, it is very difficult to perform an exact instability analysis. Instead we relax some of the usual assumptions to explore the basic physical dependencies of the length scale that can be quantified with data. An exact analysis would be expected to produce the same dependencies, with the addition of other nondimensional parameters involving the ratios of layer depths to tank diameter. As long as the disturbances are relatively small compared with the tank diameter and sediment depth, the simplified analysis applies.

The most unstable wave length is found by solving the dispersion relation which is the relationship between wave number, $\mathrm{k},(\mathrm{k}=2 \pi / \lambda$, where $\lambda$ is the wavelength $)$ of the disturbance and its growth rate, $\mathrm{n}$. The dispersion relation is obtained by performing a stability analysis on the governing differential equations of hydrodynamics. We derive a useful relation from Chandrasekhar's (1981) dispersion relation for uniform, semi-infinite, planar layers by taking advantage of two characteristics of buoyant sediment layers: 1) the liquid viscosity is much less than that of the sediment and 2) the densities of the two layers are nearly equal when an eruption begins. The dispersion relation of Chandrasekhar (1981) for a Newtonian fluid with density $\rho_{L}$ and kinematic viscosity $v_{\mathrm{L}}$ overlying a similar layer of lighter fluid (representing the sediment layer including trapped gas bubbles) of density $\rho_{B}$ and kinematic viscosity $v_{B}$, is given as follows: 


$$
\begin{aligned}
& -\mathrm{gk}_{\mathrm{n}^{2}}\left(\mathrm{p}_{\mathrm{B}}-\mathrm{p}_{\mathrm{L}}\right)+1 \mid\left(\mathrm{p}_{\mathrm{L}} \mathrm{q}_{\mathrm{B}}+\mathrm{p}_{\mathrm{B}} \mathrm{q}_{\mathrm{L}}-\mathrm{k}\right)-4 \mathrm{kp}_{\mathrm{B}} \mathrm{p}_{\mathrm{L}}+ \\
& +\frac{4 \mathrm{k}^{2}}{\mathrm{n}}\left(\mathrm{p}_{\mathrm{B}} \mathrm{v}_{\mathrm{B}}-\mathrm{p}_{\mathrm{L}} \mathrm{v}_{\mathrm{L}}\right)\left[\left(\mathrm{p}_{\mathrm{L}} \mathrm{q}_{\mathrm{B}}-\mathrm{p}_{\mathrm{B}} \mathrm{q}_{\mathrm{L}}\right)+\mathrm{k}\left(\mathrm{p}_{\mathrm{B}}-\mathrm{p}_{\mathrm{L}}\right)\right]+ \\
& +\frac{4 \mathrm{k}^{3}}{\mathrm{n}^{2}}\left(\mathrm{p}_{\mathrm{B}} \mathrm{v}_{\mathrm{B}}-\mathrm{p}_{\mathrm{L}} \mathrm{v}_{\mathrm{L}}\right)^{2}\left(\mathrm{q}_{\mathrm{B}}-\mathrm{k}\right)\left(\mathrm{q}_{\mathrm{L}}-\mathrm{k}\right)=0
\end{aligned}
$$

where

$$
\begin{aligned}
& p_{B}=\frac{\rho_{B}}{\rho_{B}+\rho_{L}}, q_{B}=\sqrt{k^{2}+\frac{n}{v_{B}}} \\
& p_{L}=\frac{\rho_{L}}{\rho_{B}+\rho_{L}}, q_{L}=\sqrt{k^{2}+\frac{n}{v_{L}}}
\end{aligned}
$$

The dispersion relation can be non-dimensionalized by introducing the characteristic length and time scales and dimensionless groups as follows:

$$
\begin{gathered}
\mathrm{d}^{3}=8 v_{\mathrm{B}} \mathrm{p}_{\mathrm{B}}^{2} / \mathrm{g}, \quad \mathrm{t}=\mathrm{d}^{2} \mathrm{v}_{\mathrm{B}} \\
\mathrm{k}_{0}=\mathrm{dk}, \quad \mathrm{n}_{0}=\mathrm{tn}, \quad \varepsilon^{2}=\mathrm{v}_{\mathrm{L}} / \mathrm{v}_{\mathrm{B}}
\end{gathered}
$$

Applying Eq. (A.3) and (A.4), the dispersion relation becomes

$$
\begin{aligned}
& -\left[\frac{8 \mathrm{k}_{0} \mathrm{p}_{\mathrm{B}}^{2}}{\mathrm{n}_{0}^{2}}\left(\mathrm{p}_{\mathrm{B}}-\mathrm{p}_{\mathrm{L}}\right)+1\right]\left(\varepsilon \mathrm{p}_{\mathrm{L}} \mathrm{q}_{\mathrm{B}}+\mathrm{p}_{\mathrm{B}} \mathrm{q}_{\mathrm{L}}-\varepsilon \mathrm{k}_{0}\right)-4 \varepsilon \mathrm{k}_{0} \mathrm{p}_{\mathrm{B}} \mathrm{p}_{\mathrm{L}}+ \\
& +\frac{4 \mathrm{k}_{0}^{2}}{\mathrm{n}_{0}}\left(\mathrm{p}_{\mathrm{B}}-\varepsilon^{2} \mathrm{p}_{\mathrm{L}}\right)\left[\left(\varepsilon \mathrm{p}_{\mathrm{L}} \mathrm{q}_{\mathrm{B}}-\mathrm{p}_{\mathrm{B}} \mathrm{q}_{\mathrm{L}}\right)+\varepsilon \mathrm{k}_{0}\left(\mathrm{p}_{\mathrm{B}}-\mathrm{p}_{\mathrm{L}}\right)\right]+ \\
& +\frac{4 \mathrm{k}_{0}^{3}}{\mathrm{n}_{0}^{2}}\left(\mathrm{p}_{\mathrm{B}}-\varepsilon^{2} \mathrm{p}_{\mathrm{L}}\right)^{2}\left(\mathrm{q}_{\mathrm{B}}-\mathrm{k}_{0}\right)\left(\mathrm{q}_{\mathrm{L}}-\varepsilon \mathrm{k}_{0}\right)=0
\end{aligned}
$$

where $\mathrm{q}_{\mathrm{B}}=\sqrt{\mathrm{k}_{0}^{2}+\mathrm{n}_{0}}$ and $\mathrm{q}_{\mathrm{L}}=\sqrt{\varepsilon^{2} \mathrm{k}_{0}^{2}+\mathrm{n}_{0}}$.

Because the sediment layer is much more viscous than the liquid layer above it, we take the limit $\varepsilon=0$. With this simplification, Eq. (A.5) becomes

$$
-8 \mathrm{k}_{0} \mathrm{p}_{\mathrm{B}}^{2}\left(\mathrm{p}_{\mathrm{B}}-\mathrm{p}_{\mathrm{L}}\right)-\mathrm{n}_{0}^{2}-4 \mathrm{k}_{0}^{2} \mathrm{n}_{0} \mathrm{p}_{\mathrm{B}}+4 \mathrm{k}_{0}^{3} \mathrm{p}_{\mathrm{B}}\left(\mathrm{q}_{\mathrm{B}}-\mathrm{k}_{0}\right)=0
$$


At the onset of buoyant instability, the densities of the two layers will be very close by definition. Hence we consider the limit of $p_{L}-p_{B}<<1$. Defining $\delta=p_{L}-p_{B}$, which implies $\mathrm{p}_{\mathrm{B}}=(1-\delta) / 2$, Eq. (A.6) becomes

$$
2 \delta(1-\delta)^{2} \mathrm{k}_{0}-\mathrm{n}_{0}^{2}-2(1-\delta) \mathrm{k}_{0}^{2} \mathrm{n}_{0}+2(1-\delta) \mathrm{k}_{0}^{3}\left(\mathrm{q}_{\mathrm{B}}-\mathrm{k}_{0}\right)=0
$$

A dominant balance occurs for $\delta<<1$ when $\mathrm{k}_{0}=\mathrm{O}\left(\delta^{1 / 3}\right)$ and $\mathrm{n}_{0}=\mathrm{O}\left(\delta^{2 / 3}\right)$. Thus we let

$$
\mathrm{k}_{0}=\delta^{1 / 3} \mathrm{~K} \text { and } \mathrm{n}_{0}=\delta^{2 / 3} \mathrm{~N}
$$

Then the dispersion relation becomes (to leading order)

$$
2 \mathrm{~K}-\mathrm{N}^{2}-2 \mathrm{~K}^{2} \mathrm{~N}+2 \mathrm{~K}^{3}\left(\sqrt{\mathrm{K}^{2}+\mathrm{N}}-\mathrm{K}\right)=0
$$

We solve this equation numerically. There is a unique value of $\mathrm{N}$ for each positive value of $\mathrm{K}$. The maximum value of $\mathrm{N}$ occurs when $\mathrm{K} \approx 1$. This corresponds to the most unstable disturbance. The values observed in the numerical solution of the exact dispersion relation, Eq. (A.7), are in the range of $1.0 \leq \mathrm{K} \leq 1.02$ for $0 \leq \delta \leq 0.06$. This is consistent with the approximation being correct to $\mathrm{O}(\delta)$ and $\mathrm{O}(\varepsilon)$.

By taking the solution approximating Eq. (A.9) to be $\mathrm{K}=\mathrm{c}_{1} \approx 1$, we can solve for the most unstable disturbance wave number, $k_{\max }$. Using the definitions given by Eq. (A.3), (A.4), and (A.8), we have

$$
\mathrm{k}_{\max }=\frac{\mathrm{c}_{1}}{2}\left[\frac{\mathrm{g}\left(\rho_{\mathrm{L}}^{2}-\rho_{\mathrm{B}}^{2}\right)}{\mu_{\mathrm{B}}^{2}}\right]^{1 / 3}
$$

which gives the wave number of the most unstable disturbance in the stratified layer. The wave number depends on the viscosity of the sediment layer, the difference in the squares of the densities of the two layers, and the gravitational constant. Now, if we take the characteristic length scale, $L$, of the gob as one-half the wavelength of the most unstable disturbance, noting that $\lambda=2 \pi / \mathrm{k}$, we have $\mathrm{L}=\pi / \mathrm{k}$ or

$$
\mathrm{L}=\frac{2 \pi}{\mathrm{c}_{1}}\left[\frac{\mu_{\mathrm{B}}^{2}}{\mathrm{~g}\left(\rho_{\mathrm{L}}^{2}-\rho_{\mathrm{B}}^{2}\right)}\right]^{1 / 3}
$$

The stability analysis has provided a simple relationship between the characteristic gob size and average waste properties. 


\section{A.2 Sediment Strength Effects on Initial Instability}

Eq. (A.11) suggests that the characteristic size of a buoyant gob depends on the difference in the densities of the two layers. If the densities are the same, Eq. (A.11) becomes singular, implying that all disturbances are equally unstable. For Eq. (A.11) to be meaningful, the bulk density of the sediment layer must be less than the liquid density. The strength of the sediment requires a slightly higher amount of gas accumulation than just necessary for neutral buoyancy before a buoyant eruption can occur. Thus the density of the sediment will be slightly lower than that of the liquid at the onset of instability.

The effects of strength on the buoyancy of plane gas-retaining layers were examined by Meyer et al. (1997). They found from elementary mechanics that the critical gas fraction required for buoyancy is given by

$$
\alpha_{C}=\alpha_{N B}+\frac{\beta \tau_{y}}{\rho_{S} g H_{S}}
$$

where $\tau_{\mathrm{y}}$ is the shear yield stress of the layer, $\mathrm{H}_{\mathrm{S}}$ is the layer thickness, and $\beta$ is a parameter relating the yield stress in shear to that in tension $(1 \leq \beta \leq \sqrt{ } 3)$.

Eq. (A.12) satisfies the requirement that the critical gas fraction required an eruption is always greater than the neutral buoyancy gas fraction when the sediment has a finite strength. This has been confirmed experimentally by Gauglitz (Stewart et al. 1996) who performed smallscale experiments using a bentonite clay sediment layer covered by water. Gas built up in the clay by decomposition of hydrogen peroxide until an eruption occurred. Table A.1 shows the relevant experimental conditions. In Figure A.1 the increase in gas fraction above neutral buoyancy from Gauglitz's work is plotted against the dimensionless group, $\tau_{y} / \rho_{S} g H_{S}$. The dashed line is Eq. (A.12) with $\beta=1.35$. The data show that the dimensionless group in Eq. (A.12) reasonably captures the elevation in gas fraction resulting from sediment strength. Variations from linear behavior are thought to be due to wall effects in the experiments. At full scale, the stabilizing influence of material strength on a plane layer is much less than in a small vessel because the surface-to-volume ratio is much smaller. This makes the critical gas fraction closer to the neutral buoyancy value.

Table A.1. Critical Void Fractions from Experiments with Clay Sediment Layers

\begin{tabular}{|c|c|c|c|c|c||}
\hline $\begin{array}{c}\mathbf{H}_{\mathbf{S}} \\
{[\mathbf{c m}]}\end{array}$ & $\begin{array}{c}\rho_{\mathbf{S}} \\
{\left[\mathbf{k g} / \mathbf{m}^{3}\right]}\end{array}$ & $\begin{array}{c}\tau_{\mathbf{y}} \\
{[\mathbf{P a}]}\end{array}$ & $\boldsymbol{\alpha}_{\mathbf{N B}}$ & $\boldsymbol{\alpha}_{\mathbf{C}}$ & $\tau / \rho_{\mathbf{S g}} \mathbf{H}_{\mathbf{S}}$ \\
\hline 4.7 & 1087 & 67 & 0.080 & 0.25 & 0.134 \\
\hline 4.8 & 1070 & 14 & 0.065 & 0.14 & 0.028 \\
\hline 1.5 & 1070 & 14 & 0.065 & 0.19 & 0.089 \\
\hline
\end{tabular}




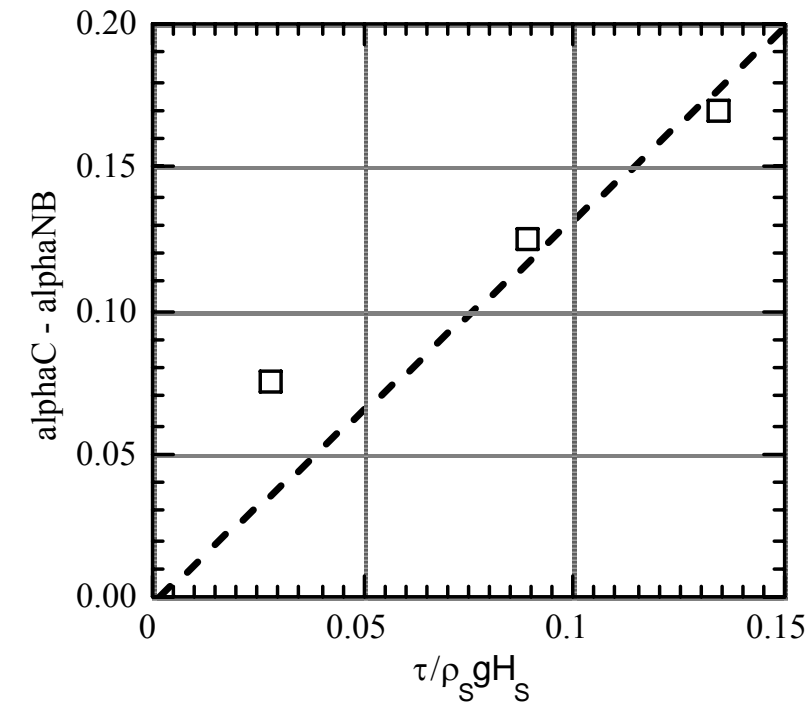

Figure A.1. Void Fraction Increase due to Sediment Strength

\section{A.3 Size of Gas Releases}

Eq. (A.12) gives the initial density difference between the sediment layer and the liquid layer at the onset of instability. This allows us to solve Eq. (A.11) for the gob length scale. Because the bulk sediment density at the onset of an eruption is given by $\rho_{B}=\left(1-\alpha_{C}\right) \rho_{S}$, we can write Eq. (A.12) as

$$
\rho_{\mathrm{B}}=\rho_{\mathrm{S}}\left(1-\alpha_{\mathrm{NB}}-\gamma\right)
$$

where

$$
\gamma=\beta \tau_{\mathrm{y}} / \rho_{\mathrm{S}} \mathrm{gH} \mathrm{S}_{\mathrm{S}}
$$

By using Eq. (A.13) with Eq. (1.1) from Section $1, \rho_{B}\left(1-\alpha_{N B}\right)=\rho_{L}$, we can write

$$
\rho_{\mathrm{L}}^{2}-\rho_{\mathrm{B}}^{2}=\gamma \rho_{\mathrm{S}}^{2}\left[2\left(1-\alpha_{\mathrm{NB}}\right)-\gamma\right]
$$

Substituting Eq. (A.15) into Eq. (A.11) and absorbing $\beta$ into the constant yields

$$
\mathrm{L}=\frac{2 \pi}{\mathrm{c}_{1}}\left[\frac{\mu_{\mathrm{B}}^{2} \mathrm{H}_{\mathrm{S}}}{\rho_{\mathrm{S}} \tau_{\mathrm{y}}}\right]^{1 / 3}\left[2\left(1-\alpha_{\mathrm{NB}}\right)-\gamma\right]^{1 / 3}
$$


Equation (A.16) gives the gob length scale in terms of the properties of the sediment layer. We make the assumption that the gob volume, $\mathrm{V}_{0}$, scales with $\mathrm{L}^{3}$, where the constant of proportionality is determined experimentally. Hence we write

$$
\mathrm{V}_{0} \approx \mathrm{cL}^{3}=\mathrm{c} \frac{\mu_{\mathrm{B}}^{2} \mathrm{H}_{\mathrm{S}}}{\rho_{\mathrm{S}} \tau_{\mathrm{y}}}\left[2\left(1-\alpha_{\mathrm{NB}}\right)-\gamma\right]^{1}
$$

The amount of gas, $V_{R}$, released during the eruption is determined by the amount of gas stored in the gob $\left(\alpha_{c} V_{0}\right)$ and the fraction that is released during the eruption process. The gas release fraction, $\Phi$, can be estimated by assuming that gas is released until the gob, which is now near the surface of the waste, returns to neutral buoyancy and sinks back to rejoin the sediment layer. The eruption begins with a gob of gas fraction $\alpha_{C}$ at a pressure $p$ and ends at a gas fraction of $\alpha_{N B}$ at atmospheric pressure $p_{A}$. Using the definition of the gas volume fraction, the release fraction can be expressed as

$$
\Phi=1-\frac{\alpha_{N B}\left(1-\alpha_{C}\right)}{\alpha_{C}\left(1-\alpha_{N B}\right)} \frac{p_{A}}{p}
$$

Hence the total amount of gas released during a buoyant eruption is

$$
\begin{aligned}
\mathrm{V}_{\mathrm{R}} & =\alpha_{\mathrm{C}} \Phi \mathrm{V}_{0} \frac{\mathrm{p}}{\mathrm{p}_{\mathrm{A}}} \\
& =\mathrm{c}\left(\alpha_{\mathrm{NB}}+\gamma\right)\left[1-\frac{\alpha_{\mathrm{NB}}\left(1-\alpha_{\mathrm{NB}}-\gamma\right)}{\left(\alpha_{\mathrm{NB}}+\gamma\right)\left(1-\alpha_{\mathrm{NB}}\right)} \frac{\mathrm{p}_{\mathrm{A}}}{\mathrm{p}}\right] \frac{\mathrm{p}}{\mathrm{p}_{\mathrm{A}}} \frac{\mu_{\mathrm{B}}^{2} \mathrm{H}_{\mathrm{S}}}{\rho_{\mathrm{S}} \tau_{\mathrm{y}}}\left[2\left(1-\alpha_{\mathrm{NB}}\right)-\gamma\right]^{1}
\end{aligned}
$$

In the weak sediment limit the parameter $\gamma=\beta \tau_{\mathrm{y}} / \rho_{\mathrm{S}} \mathrm{gH} \mathrm{S}_{\mathrm{S}}<<1$. Also, in sediment with a finite strength the apparent viscosity can be assumed proportional to the yield stress. These assumptions allow Eq. (A.19) to be simplified considerably as follows:

$$
\mathrm{V}_{\mathrm{R}} \approx \mathrm{C} \frac{\alpha_{\mathrm{NB}}}{1-\alpha_{\mathrm{NB}}}\left(\frac{\mathrm{p}}{\mathrm{p}_{\mathrm{A}}}-1\right) \frac{\tau_{\mathrm{y}} \mathrm{H}_{\mathrm{S}}}{\rho_{\mathrm{S}}}
$$

It is important to emphasize that the model predicts the most probable gob size, not the largest or smallest. The largest possible gob, in principle, is one with diameter equal to the tank diameter because all scales are potentially unstable. However the historical gas release data indicate a maximum gob size approximately double the most probable size. 


\section{A.4 References}

Chandrasekhar S. 1981. Hydrodynamic and Hydromagnetic Stability. Dover Publications, Inc., New York.

Meyer PA, ME Brewster, SA Bryan, G Chen, LR Pederson, CW Stewart, and G Terrones. 1997. Gas Retention and Release Behavior in Hanford Double Shell Waste Tanks. PNNL-11536 Rev. 1, Pacific Northwest National Laboratory, Richland, Washington.

Stewart CW, JM Alzheimer, ME Brewster, G Chen, RE Mendoza, HC Reid, CL Shepard, and G Terrones. 1996. In Situ Rheology and Gas Volume in Hanford Double-Shell Waste Tanks. PNNL-11296, Pacific Northwest National Laboratory, Richland, Washington. 
Appendix B

\section{Waste Layer Density Data}




\section{Appendix B - Waste Layer Density Data}

Layer density results for $\mathrm{AN}-104, \mathrm{AN}-105, \mathrm{AW}-101$, and $\mathrm{SY}-103$ are summarized in Tables B.1 through B.4. 
Table B.1. Layer Density Results for AN-104

\begin{tabular}{|c|c|c|c|c|c|c|c|c|c|}
\hline Constituent & $\begin{array}{c}\text { Core, } \\
\text { Segment }\end{array}$ & Riser & Date & Median & Mean & Max & Min & $\begin{array}{l}\text { Standard } \\
\text { Deviation }\end{array}$ & Data Points \\
\hline Liquid SpG & $\begin{array}{c}163 \mathrm{~S} 2,3-12 \\
164 \mathrm{~S} 1-13\end{array}$ & $10 \mathrm{~A}, 12 \mathrm{~A}$ & 1996 & 1.406 & 1.400 & 1.49 & 1.339 & 0.033835 & 48 \\
\hline Liquid SpG & $282, \mathrm{~S} 1,15,20$ & 22 & 2000 & 1.499 & 1.500 & 1.515 & 1.479 & 0.014838 & 5 \\
\hline $\begin{array}{l}\text { Liquid } \\
\text { Density }\end{array}$ & $\begin{array}{c}163 \mathrm{~S} 2,4-11,14 \\
164 \mathrm{~S} 2-13\end{array}$ & $10 \mathrm{~A}, 12 \mathrm{~A}$ & 1996 & 1.46 & 1.435 & 1.54 & 1.32 & 0.066228 & 21 \\
\hline $\begin{array}{l}\text { Liquid } \\
\text { Density }\end{array}$ & Ball Rheometer & $1 \mathrm{~B}, 16 \mathrm{~B}$ & 1996 & 1.44 & 1.443 & 1.5 & 1.4 & 0.03199 & 10 \\
\hline $\mathrm{mIL} / \mathrm{VIL}$ & $282, \mathrm{~S} 15$ & 22 & 2000 & 1.247059 & & & & & 1 \\
\hline $\mathrm{mIL} / \mathrm{VIL}$ & 282, S20 & 22 & 2000 & 0.972222 & & & & & 1 \\
\hline $\mathrm{mDL} / \mathrm{VDL}$ & $282, \mathrm{~S} 1$ & 22 & 2000 & 1.511 & & & & & 1 \\
\hline $\mathrm{mDL} / \mathrm{VDL}$ & 282, S01R & 22 & 2000 & 1.512982 & & & & & 1 \\
\hline $\begin{array}{l}\text { NCL Solid } \\
\text { Density }\end{array}$ & $\begin{array}{c}163 \mathrm{~S} 16,18,20 \\
164 \mathrm{~S} 13-17,19,20\end{array}$ & $10 \mathrm{~A}, 12 \mathrm{~A}$ & 1996 & 1.570 & 1.578 & 1.710 & 1.520 & 0.045 & 19 \\
\hline $\begin{array}{l}\text { NCL Solid } \\
\text { Density }\end{array}$ & $282, \mathrm{~S} 11$ & 22 & 2000 & 1.552392 & 1.533603 & 1.577778 & 1.451852 & 0.056219 & 4 \\
\hline $\mathrm{mCS} / \mathrm{VCS}$ & $282, \mathrm{~S} 15$ & 22 & 2000 & 1.6 & & & & & 1 \\
\hline $\mathrm{mCS} / \mathrm{VCS}$ & 282, S20 & 22 & 2000 & 1.630769 & & & & & 1 \\
\hline $\begin{array}{l}\text { Solid Comp } \\
\text { Bulk Density }\end{array}$ & 164 & $12 \mathrm{~A}$ & 1996 & 1.57 & & & & & 1 \\
\hline Cent Solids & $282, \mathrm{~S} 15$ & 22 & 2000 & 1.59 & & & & & 1 \\
\hline Cent Solids & 282, S20 & 22 & 2000 & 1.63 & & & & & 1 \\
\hline
\end{tabular}


Table B.2. Layer Density Results for AN-105

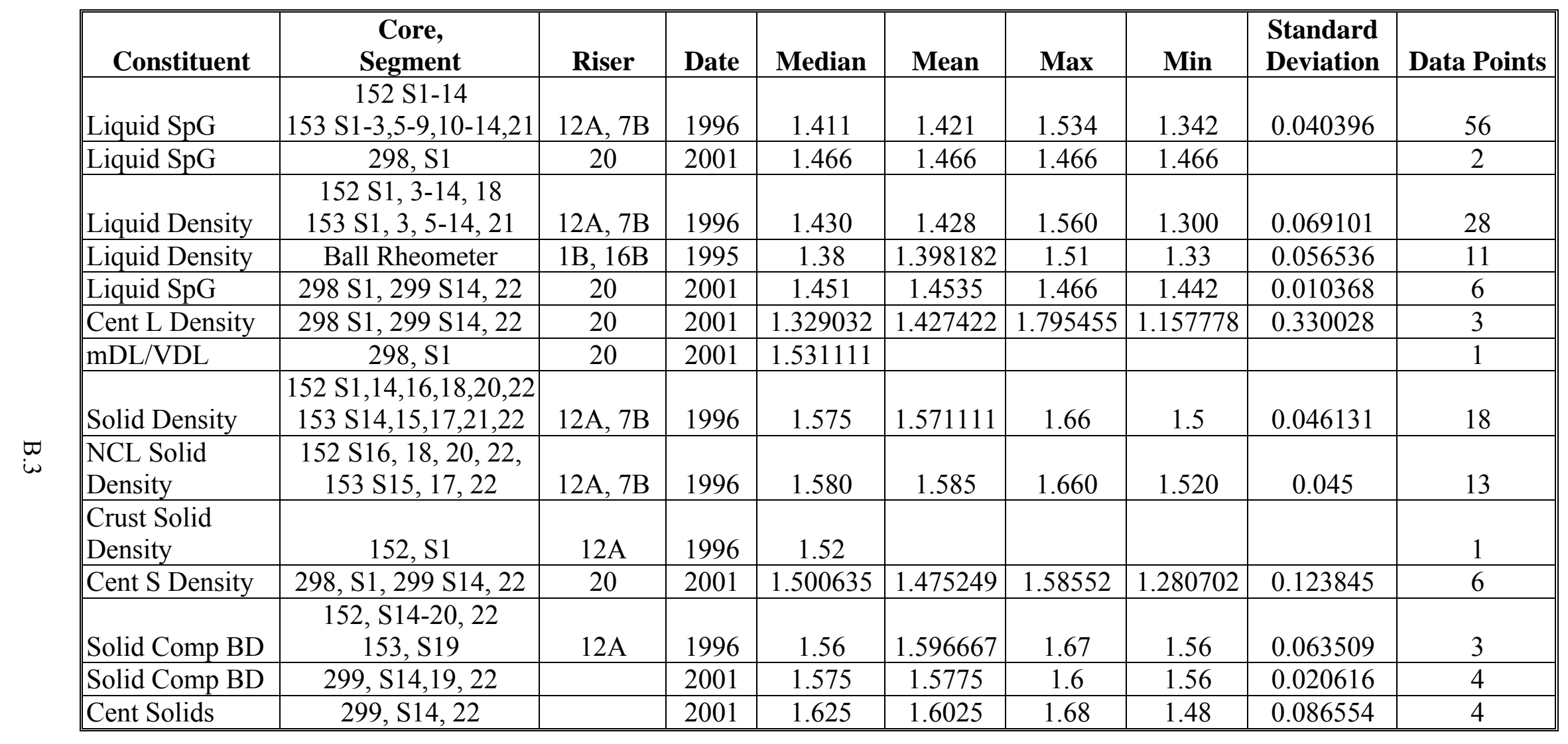


Table B.3. Layer Density Results for AW-101

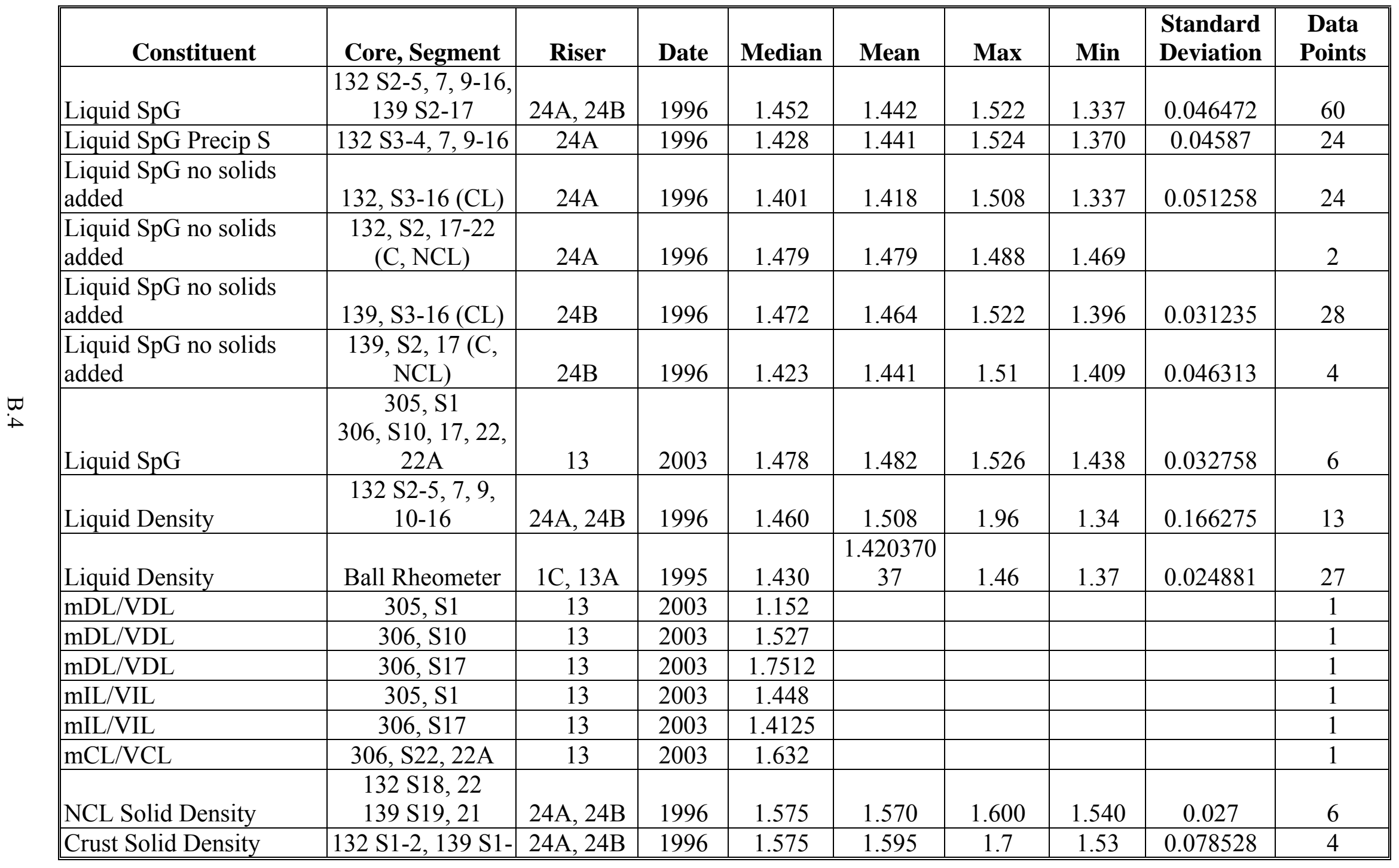


Table B.3. Layer Density Results for AW-101

\begin{tabular}{|c|c|c|c|c|c|c|c|c|c|}
\hline Constituent & Core, Segment & Riser & Date & Median & Mean & Max & Min & $\begin{array}{l}\text { Standard } \\
\text { Deviation }\end{array}$ & $\begin{array}{c}\text { Data } \\
\text { Points }\end{array}$ \\
\hline & 2 & & & & & & & & \\
\hline Precip Solid Density & $\begin{array}{c}132 \mathrm{~S} 5,10,15 \\
139 \mathrm{~S} 5,16\end{array}$ & $24 \mathrm{~A}, 24 \mathrm{~B}$ & 1996 & 1.700 & 1.696 & 1.86 & 1.54 & 0.116748 & 5 \\
\hline mCS/VCS, crust & $305, \mathrm{~S} 1$ & 13 & 2003 & 1.580 & & & & & 1 \\
\hline $\mathrm{mCS} / \mathrm{VCS}, \mathrm{NCL}$ & $306, \mathrm{~S} 17,22,22 \mathrm{~A}$ & 13 & 2003 & 1.555 & 1.555 & 1.56 & 1.55 & & 2 \\
\hline $\begin{array}{l}\text { Settled Solids density, } \\
\text { crust }\end{array}$ & $305, \mathrm{~S} 1$ & 13 & 2003 & 1.540 & & & & & 1 \\
\hline $\begin{array}{l}\text { Settled Solids density, } \\
\text { NCL }\end{array}$ & $306, \mathrm{~S} 17,22,22 \mathrm{~A}$ & 13 & 2003 & 1.540 & 1.540 & 1.57 & 1.51 & & 2 \\
\hline $\begin{array}{l}\text { SpG LAW Supernate } \\
\text { Composite }\end{array}$ & Grab Sample & 22 & 1998 & 1.467 & 1.47 & 1.484 & 1.459 & 0.012767 & 3 \\
\hline SpG WTC & Grab Sample & "NA" & 1991 & 1.54 & 1.54 & 1.54 & 1.54 & & 2 \\
\hline $\begin{array}{l}\text { SpG Grab Sample 1AW- } \\
\text { 00-7 (sup Liq) }\end{array}$ & Grab Sample & 22 & 2000 & 1.4705 & 1.4705 & 1.473 & 1.468 & & 2 \\
\hline Seg solids BD & $132, \mathrm{~S} 7$ & $24 \mathrm{~A}$ & 1996 & 1.54 & & & & & 1 \\
\hline $\begin{array}{l}\text { Stratum Comp. (crust } \\
\text { Comp, saltc Sol) }\end{array}$ & 132 & $24 \mathrm{~A}$ & 1996 & 1.62 & & & & & 1 \\
\hline $\begin{array}{l}\text { Stratum Comp. (CL } \\
\text { Comp, saltc Sol) }\end{array}$ & 132 & $24 \mathrm{~A}$ & 1996 & 1.68 & & & & & 1 \\
\hline $\begin{array}{l}\text { Stratum Comp. (NCL } \\
\text { Comp, saltc Sol) }\end{array}$ & 132 & $24 \mathrm{~A}$ & 1996 & 1.58 & & & & & 1 \\
\hline Liquid Grab Sample & Grab Sample & $16 \mathrm{C}$ & 1990 & 1.51 & & & & & 1 \\
\hline Liquid Grab Sample & Grab Sample & $22 \mathrm{~A}$ & 1990 & 1.57 & & & & & 1 \\
\hline $\begin{array}{l}\text { Settled Solids Grab } \\
\text { Sample }\end{array}$ & Grab Sample & 22 & 2000 & 1.625 & 1.625 & 1.64 & 1.61 & & 2 \\
\hline
\end{tabular}


Table B.4. Layer Density Results for SY-103

\begin{tabular}{|c|c|c|c|c|c|c|c|c|c|}
\hline Constituent & $\begin{array}{c}\text { Core, } \\
\text { Segment }\end{array}$ & Riser & Date & Median & Mean & Max & Min & $\begin{array}{l}\text { Standard } \\
\text { Deviation }\end{array}$ & $\begin{array}{c}\text { Data } \\
\text { Points }\end{array}$ \\
\hline Liquid SpG composite & 280 & 21 & 2000 & 1.47 & 1.469 & 1.471 & 1.466 & 0.002646 & 3 \\
\hline Liquid density & $62 \mathrm{~S} 1-8,15$ & $14 \mathrm{~A}$ & 1994 & 1.48 & 1.462 & 1.53 & 1.31 & 0.075689 & 10 \\
\hline Liquid density precip sol & $62, \mathrm{~S} 2-8$ & $14 \mathrm{~A}$ & 1994 & 1.482 & 1.476 & 1.529 & 1.352 & 0.054616 & 8 \\
\hline Liquid density & $\begin{array}{c}279 \mathrm{~S} 1-8,280 \\
\text { S1-9 }\end{array}$ & 17,21 & 2000 & 1.480333 & 1.464 & 1.544483 & 1.318462 & 0.063313 & 17 \\
\hline Liquid density & Ball rheometer & $17 \mathrm{C}, 22 \mathrm{~A}$ & 1995 & 1.48 & 1.474 & 1.52 & 1.43 & 0.032587 & 7 \\
\hline Stratum A comp. (crust) & $62, \mathrm{~S} 1$ & $14 \mathrm{~A}$ & 1994 & 1.59 & & & & & 1 \\
\hline $\begin{array}{l}\text { Stratum B comp. (sup } \\
\text { seg seeCORE) }\end{array}$ & $62,4-8$ & $14 \mathrm{~A}$ & 1994 & 1.51 & & & & & 1 \\
\hline Stratum C comp. (NCL) & $62, \mathrm{~S} 9$ & $14 \mathrm{~A}$ & 1994 & 1.51 & & & & & 1 \\
\hline Stratum D comp. (NCL) & $62,10-14$ & $14 \mathrm{~A}$ & 1994 & 1.57 & & & & & 1 \\
\hline Comp. solids & $279, \mathrm{~S} 8-14$ & 17 & 2000 & 1.625 & 1.626667 & 1.634 & 1.621 & 0.006658 & 3 \\
\hline $\begin{array}{l}\text { Comp. solids (cent. } \\
\text { solids) }\end{array}$ & $280, \mathrm{~S} 1-10$ & 21 & 2000 & 1.544 & 1.546667 & 1.57 & 1.526 & 0.022121 & 3 \\
\hline Comp. solids & 280, S9-15 & 21 & 2000 & 1.588 & 1.591667 & 1.602 & 1.585 & 0.009074 & 3 \\
\hline $\begin{array}{l}\text { Solubility comp. cent } \\
\text { liquid }\end{array}$ & 279 & 17 & 2000 & 1.499 & & & & & 1 \\
\hline $\begin{array}{l}\text { Solubility comp. cent } \\
\text { solids }\end{array}$ & 279 & 17 & 2000 & 1.68 & & & & & 1 \\
\hline $\begin{array}{l}\text { Solubility comp. cent } \\
\text { liquid }\end{array}$ & 280 & 21 & 2000 & 1.485 & & & & & 1 \\
\hline $\begin{array}{l}\text { Solubility comp. cent } \\
\text { solids }\end{array}$ & 280 & 21 & 2000 & 1.62 & & & & & 1 \\
\hline
\end{tabular}




\section{Appendix C}

Cumulative Distribution Plots for

Sediment Density Comparisons 


\section{Appendix C - Cumulative Distribution Plots for Sediment Density Comparisons}

Sediment layer density data for AN-104, AN-105, AW-101, and SY-103 are compared with the computed density in Figures C.1 through C.5. As described in Section 3.2.2 of the main report, the larger standard deviations of the data are not unexpected given the apparent effect of gas retention displacing liquid from the sediment. This effect would expand the sediment density range, suggesting an associated large variance in the solid volume fraction.

$\mathrm{AN}-103$ is the most extreme example, and this is not unexpected given its large retained gas volume (Hedengren et al. 2000). In addition, extreme values are not expected to necessarily be representative of the bulk of the sediment. Thus, it is postulated that the computed sediment densities are a reasonable representation of the bulk degassed sediment.

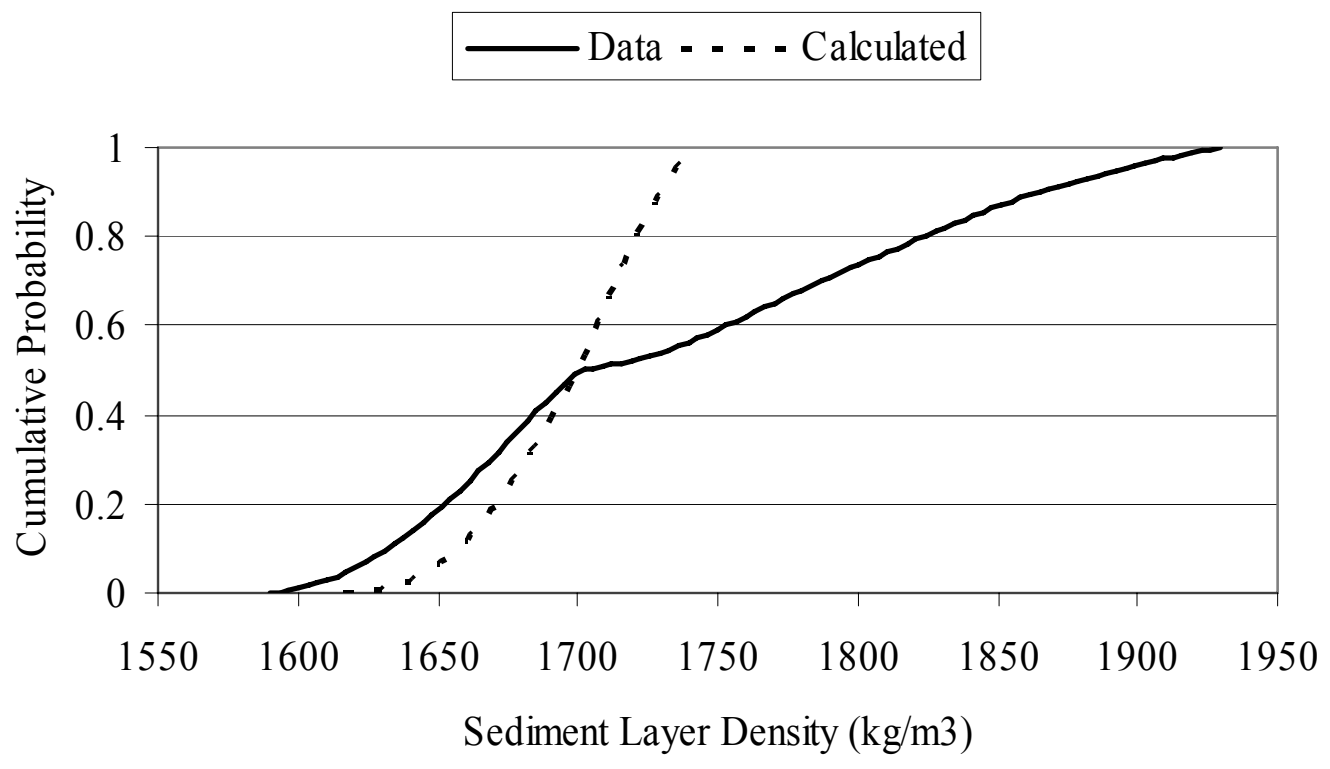

Figure C.1. Data and Calculated Sediment Density Comparison for AN-103 


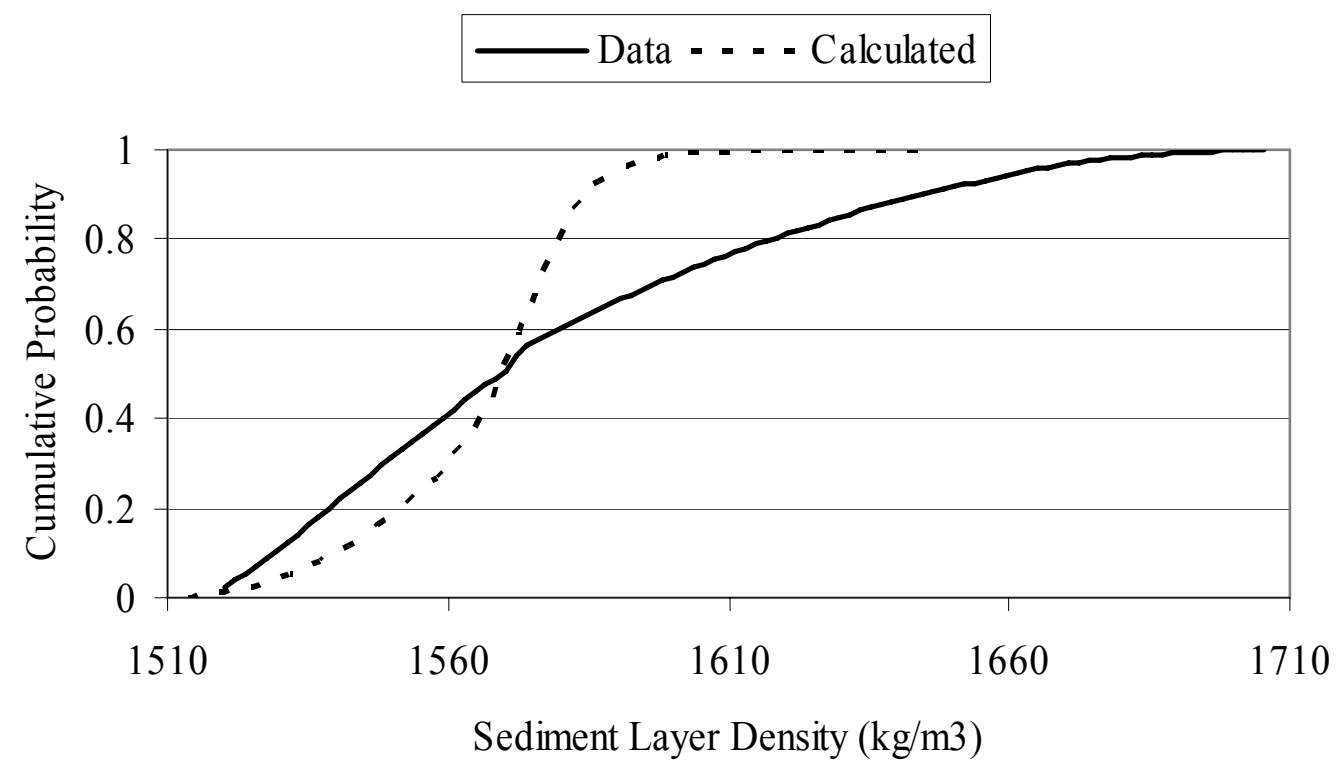

Figure C.2. Data and Calculated Sediment Density Comparison for AN-104

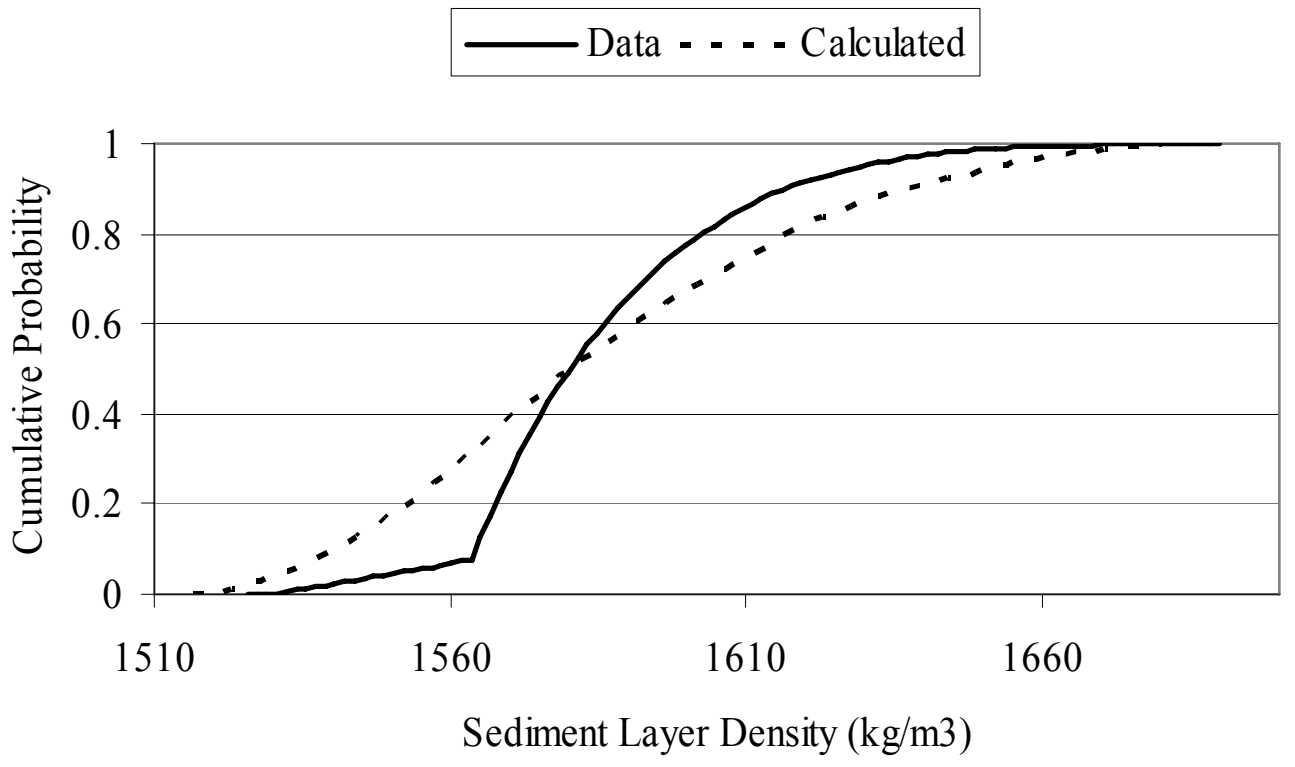

Figure C.3. Data and Calculated Sediment Density Comparison for AN-105 


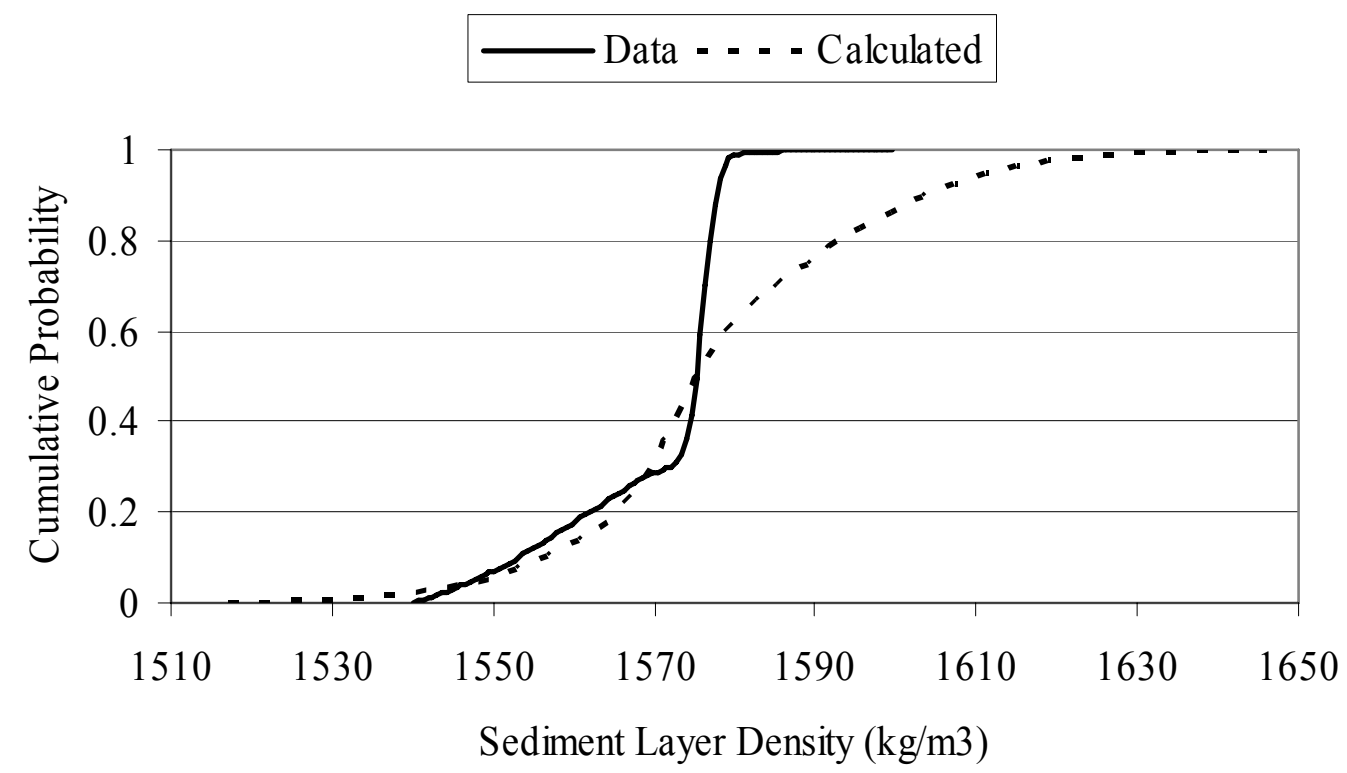

Figure C.4. Data and Calculated Sediment Density Comparison for AW-101

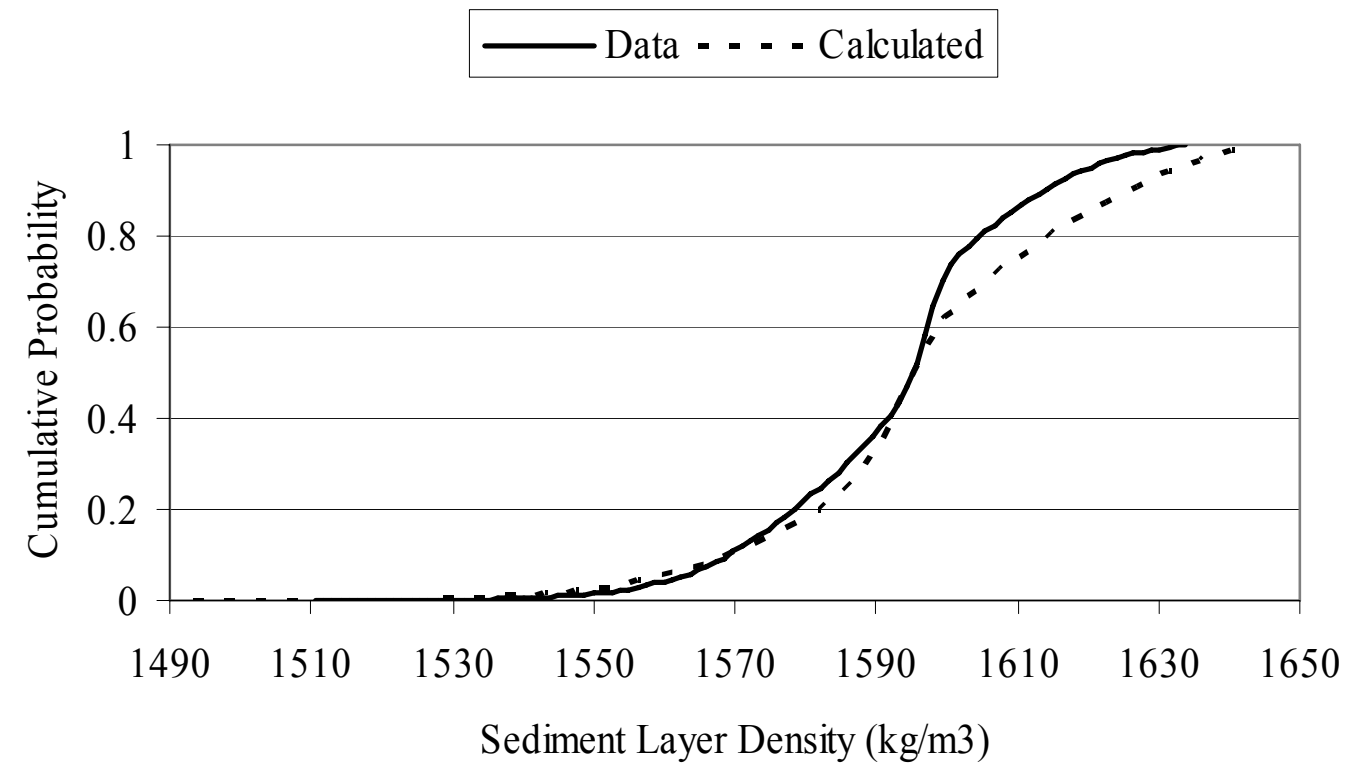

Figure C.5. Data and Calculated Sediment Density Comparison for SY-103

\section{Reference}

Hedengren DC, KM Hodgson, WB Barton, CW Stewart, JM Cuta, and BE Wells. 2000. Data and Observations on Double-Shell Flammable Gas Watch List Tank Behavior. RPP-6655 Rev. 0, CH2M HILL Hanford Group, Richland, Washington. 
Appendix D

Combination, Uncertainty and Data for Sediment Layer Depth 


\section{Appendix D - Combination, Uncertainty and Data for Sediment Layer Depth}

Sediment layer depth data for Hanford tanks AN-104, AN-105, AW-101, and SY-103 are investigated in this appendix. For each tank, we have up to three different measurement methods to evaluate the sediment depth. From sampler configuration and/or application, estimates are provided as to the "believability" or "reliability/repeatability" of that measurement method, denoted as $\sigma_{\mathrm{mi}}$. The three methods are ball rheometer, core samples, and the temperature validation probes deployed in the multifunction instrument trees (MITvip).

For each source, the believability is given as one standard deviation of the total range (assumed to be normally distributed; one standard deviation is $1 / 6$ of the range) of the instrument. Layer interface identification is made with the ball rheometer by the changing buoyancy of the ball. To account for the changing buoyancy as the ball passes through the interface, the ball diameter, $10 \mathrm{~cm}$, is taken to be the range. For the core samples, two 19-inch segment lengths to identify interfaces (range is increased to 40 inches to allow for possible partially full segments). Validation probe temperature measurements are typically taken every 4 inches, and two to three readings are typically required to determine an interface so the range is estimated at 12 inches. The respective $\sigma_{\mathrm{mi}}$ for each method are thus $0.017,0.17$, and $0.051 \mathrm{~m}$.

Given this knowledge, estimated mean and median depths should be weighted according to the believability of each instrument. Determining those weights is as follows:

Let $Q=a_{1} x+a_{2} y+a_{3} z=a_{1} x+a_{2} y+\left(1-a_{1}-a_{2}\right) z$ where $a_{1}, a_{2}, a_{3}$ are the weights be determined.

Assume $x, y, z$ are all unbiased and represent the ball rheometer, core sample, and MITvip measurement methods, respectively. Then $\operatorname{Var}(\mathrm{Q})=\mathrm{a}_{1}{ }^{2} \operatorname{var}(\mathrm{x})+\mathrm{a}_{2}{ }^{2} \operatorname{var}(\mathrm{y})+\left(1-\mathrm{a}_{1}-\mathrm{a}_{2}\right)^{2} \operatorname{var}(\mathrm{z})$.

Our prior assumption is:

$\operatorname{Var}(\mathrm{x})=\sigma_{\mathrm{b}}^{2}(\mathrm{x})=0.017^{2}=0.000289$

$\operatorname{Var}(\mathrm{y})=\sigma_{\mathrm{b}}^{2}(\mathrm{y})=0.17^{2}=0.0289$

$\operatorname{Var}(\mathrm{z})=\sigma_{\mathrm{b}}^{2}(\mathrm{z})=0.051^{2}=0.002601$

We want to minimize $\operatorname{Var}(\mathrm{Q})$ such that:

$$
\begin{aligned}
& \operatorname{Var}(\mathrm{Q})=0.000289 \mathrm{a}_{1}^{2}+0.0289 \mathrm{a}_{2}^{2}+0.002601\left(1-\mathrm{a}_{1}-\mathrm{a}_{2}\right)^{2} \\
& \partial / \partial \mathrm{a}_{1}=2(0.000289) \mathrm{a}_{1}-2(0.002601)\left(1-\mathrm{a}_{1}-\mathrm{a}_{2}\right)=0.000578 \mathrm{a}_{1}-0.005202\left(1-\mathrm{a}_{1}-\mathrm{a}_{2}\right)
\end{aligned}
$$




$$
\partial / \partial \mathrm{a}_{2}=2(0.0289) \mathrm{a}_{2}-2(0.002601)\left(1-\mathrm{a}_{1}-\mathrm{a}_{2}\right)=0.0578 \mathrm{a}_{2}-0.005202\left(1-\mathrm{a}_{1}-\mathrm{a}_{2}\right)
$$

Solving for $\mathrm{a} 1$ and $\mathrm{a} 2, \mathrm{a}_{1}=0.891972$ and $\mathrm{a}_{2}=0.00891972$.

So, our weights are $\mathrm{a} 1=0.89, \mathrm{a} 2=0.01, \mathrm{a} 3=1-0.89-0.01=0.10$.

Using these weights, the estimated weighted means and medians for each tank are determined. In situations were one or more of the measurement methods were not used, the weights were re-distributed according to their relative proportion to $100 \%$.

The formula for each tank is as follows:

$$
\bar{\chi}_{\text {tank }}=\sum .89 *(\overline{\text { BallRheometer }})+.01 *(\overline{\text { CoreSample })}+.10 *(\overline{\text { MITvip })}
$$

where

$\bar{X}_{\text {Tank }}$

BallRheometer

$\overline{\text { CoreSamples }}$

$\overline{\text { MITvip }}$

$=$ weighted mean of a specific tank

$=$ mean of the ball rheometer measurements within a specific tank

$=$ mean of the core sample measurements within a specific tank

$=$ mean of the MITvip measurements within a specific tank.

The estimates for the weighted medians are calculated in the same manner, using the median of each set of measurements rather than their means. Table D.1 shows the results of the weighted mean and median calculations for each tank.

Table D.1. Weighted Mean and Median Sediment Depths by Tank

\begin{tabular}{|c|c|c|c|c|c||}
\hline Tank & $\begin{array}{c}\text { Weighted } \\
\text { Median }\end{array}$ & $\begin{array}{c}\text { Weighted } \\
\text { Mean }\end{array}$ & Tank & Weighted Median & $\begin{array}{c}\text { Weighted } \\
\text { Mean }\end{array}$ \\
\hline AN-103 & 3.79 & 3.79 & AW-101H & 2.69 & 2.69 \\
\hline AN-104 & 3.95 & 3.96 & AW-101C & 2.95 & 2.89 \\
\hline AN-105 & 4.36 & 4.36 & SY-103 & 3.27 & 3.26 \\
\hline
\end{tabular}

To estimate the variability by tank, the prior knowledge of the measurement methods, as wells as the variability inherent in the actual measurements that were taken, are used. In typical situations of this type of data, the measurement error is usually not known separately from the physical variability. That is, the variability in the data provided is composed of both variability types, and is confounded. Because we have an estimate of the measurement error, it is possible to calculate the variability of the sediment depth in each tank accordingly. The structure of the data is shown in Table D.2. 
Table D.2. Sediment Depth Data by Tank

\begin{tabular}{|c|c|c|c|}
\hline Tank & Ball Rheometer & Core Samples & MITvip \\
\hline AN103 & $3.74,3.86$ & $2.92,3.86$ & $3.64,3.71,3.71,3.71$ \\
\hline AN104 & $4.12,3.72$ & $4.4,3.65,4.6$ & $4.47,4.17,4.17,4.25$ \\
\hline AN105 & $4.55,4.05$ & $3.95,3.98$ & $4.93,4.78,5.01,4.93,4.93$ \\
\hline AW101H & $2.59,2.78$ & 2.9 & \\
\hline AW101C & & & $2.57,2.95,2.95,2.87,3.1$ \\
\hline SY103 & $3.2,3.29$ & $3.37,2.9,2.84$ & $2.57,3.556,3.71,2.95,3.25$, \\
& & & $4.09,3.64,3.1,3.56,3.71$ \\
\hline
\end{tabular}

Within each cell (a cell is defined as a Tank/Method combination):

$\sigma^{2}($ Tank, Method $)=\sigma_{l, t, i}^{2}+\sigma_{m, i}^{2}$ where $l=$ location, $t=$ tank, $m, i=$ measurement method

Therefore,

$$
\sigma^{2}(\text { Tank }, \text { Method })=\sigma_{l, t, i}^{2}+\sigma_{m, i}^{2}=\sum_{j} \frac{\left(x_{j}-\bar{x}_{j}\right)^{2}}{n_{j}}
$$

Solving for $\sigma_{l, t, i}^{2}: \sigma_{l, t, i}^{2}==\sum_{j} \frac{\left(x_{j}-\bar{x}_{j}\right)^{2}}{n_{j}}-\sigma_{m, i}^{2}$

$\sigma_{m, i}^{2}$ is given for each tank: $\sigma_{m, 1}^{2}=.017^{2}, \sigma_{m, 2}^{2}=.17^{2}, \sigma_{m, 3}^{2}=.051^{2}$

Pooling the variance of the sediment depth within a tank across measurement methods:

$$
\sigma^{2}(\operatorname{Tank})=\frac{\left(n_{1}-1\right) \sigma_{l, t, 1}^{2}+\left(n_{2}-1\right) \sigma_{l, t, 2}^{2}+\left(n_{3}-1\right) \sigma_{l, t, 3}^{2}}{n_{1}+n_{2}+n_{3}-3}
$$

For each tank, the sediment depth variability calculations are:

$$
\begin{gathered}
\sigma^{2}(\text { AN103 })=\frac{(2-1) \cdot 0069+(2-1) \cdot 4129+(4-1) 0}{2+2+4-3}=.0840 \\
\sigma(A N 103)=. \sqrt{.0840=} .29
\end{gathered}
$$




$$
\begin{aligned}
& \sigma^{2}(A N 104)=\frac{(2-1) \cdot 0797+(3-1) \cdot 2219+(4-1) \cdot 0175}{2+3+4-3}=.0960 \\
& \sigma(A N 104)=. \sqrt{.0960=} .31 \\
& \sigma^{2}(A N 105)=\frac{(2-1) \cdot 1247+(2-1) 0+(5-1) \cdot 0044}{2+2+5-3}=.0237 \\
& \sigma(A N 105)=. \sqrt{.0237}=.154 \\
& \sigma^{2}(A W 101 H)=\frac{.0178}{2-1}=.0178 * \\
& \sigma(A W 101 H)=. \sqrt{.0178}=.1332 * \\
& \sigma^{2}(A W 101 C)=\frac{.0359}{5-1}=.009 * \\
& \sigma(A W 101 C)=. \sqrt{.009=.09} * \\
& \sigma^{2}(S Y 103)=\frac{(2-1) \cdot 0038+(3-1) \cdot 0553+(10-1) \cdot 195}{2+3+10-3}=.1557 \\
& \sigma(S Y 103)=. \sqrt{.1557=} .395
\end{aligned}
$$

Note the "*" at the end of the AW101H and AW101C calculations. For both tanks, the estimates were considered too small due to the lack of data used in the calculations. Therefore, an average variability amongst all the other tanks was calculated and substituted for these two tanks. The variability used for both these tanks in the Monte Carlo simulation is 0.287.

In summary, the sediment depth weighted medians, means, variances, and standard deviations, by tank, are presented in Table D.3. 
Table D.3. Sediment Layer Depth Distributions

( $\mathrm{H}$ and $\mathrm{C}$ with tank name indicate hot and cold tank states)

\begin{tabular}{||c|c|c|c|c|c||}
\hline Tank & $\begin{array}{c}\text { Weighted } \\
\text { Median }\end{array}$ & $\begin{array}{c}\text { Weighted } \\
\text { Mean }\end{array}$ & Variance & $\begin{array}{c}\text { Standard } \\
\text { Deviation }\end{array}$ & Data Points \\
\hline AN-103 & 3.787 & 3.785 & 0.084 & 0.29 & 8 \\
\hline AN-104 & 3.954 & 3.957 & 0.096 & 0.31 & 9 \\
\hline AN-105 & 4.360 & 4.358 & 0.0237 & 0.154 & 9 \\
\hline AW-101H & 2.687 & 2.687 & 0.082 & 0.287 & 3 \\
\hline AW-101C & 2.950 & 2.888 & 0.082 & 0.287 & 5 \\
\hline SY-103 & 3.273 & 3.260 & 0.1557 & 0.395 & 15 \\
\hline
\end{tabular}

For completeness, it should be noted that the variability around the mean in each tank is a different calculation, and not what is needed for the Monte Carlo simulation and subsequent modeling. Specifically, the calculation for the mean of each tank is as follows:

$\bar{x}=\sum_{i} a_{i} \overline{x_{i, t}}$ where $\mathrm{i}=$ measurement method, $\mathrm{t}=$ tank.

$$
\underset{\operatorname{var}(\bar{x})}{=}=\sum_{i} a_{i}^{2} \operatorname{var}\left(\overline{x_{i, t}}\right)=\sum a_{i}{ }^{2} \frac{\left(\sigma_{l, t}^{2}+\sigma_{m, i}^{2}\right)}{n_{i}}=\sigma_{l, t}^{2} \frac{\sum a_{i}^{2}}{n_{i}}+\frac{\sum a_{i}^{2} \sigma_{m, i}^{2}}{n_{i}}
$$

Therefore, the variability associated with the mean of each tank is as follows:

$$
\begin{aligned}
& \sigma^{2}(\overline{\overline{A N 103}})= \\
& \left\{.083 *\left[\left(.89^{2} / 2\right)+\left(.01^{2} / 2\right)+\left(.1^{2} / 4\right)\right]\right\}+\left\{\left(.89^{2} * .017^{2}\right) / 2+\left(.01^{2} * .17^{2}\right) / 2+\left(.10^{2} * .051^{2}\right) / 4\right\}=.0336 \\
& \sigma^{2}(\overline{\overline{A N 104}})= \\
& \left\{.096 *\left[\left(.89^{2} / 2\right)+\left(.01^{2} / 3\right)+\left(.1^{2} / 4\right)\right]\right\}+\left\{\left(.89^{2} * .017^{2}\right) / 2+\left(.01^{2} * .17^{2}\right) / 3+\left(.10^{2} * .051^{2}\right) / 4\right\}=.0383 \\
& \sigma^{2}(\overline{\overline{A N 105}})= \\
& \left\{.0237 *\left[\left(.89^{2} / 2\right)+\left(.01^{2} / 2\right)+\left(.1^{2} / 5\right)\right]\right\}+\left\{\left(.89^{2} * .017^{2}\right) / 2+\left(.01^{2} * .17^{2}\right) / 2+\left(.10^{2} * .051^{2}\right) / 5\right\}=.0096 \\
& \sigma^{2}(\overline{\overline{A W 101 H}})=\left\{.0178^{*}\left[\left(1^{2} / 2\right)\right]\right\}+\left\{\left(1^{2} * .017^{2}\right) / 2\right\}=.009
\end{aligned}
$$




$$
\sigma^{2}(\overline{\overline{A W 101 C}})=\left\{.009 *\left[\left(1^{2} / 5\right)\right]\right\}+\left\{\left(1^{2 *} .051^{2}\right) / 5\right\}=.0023
$$

$$
\begin{aligned}
& \sigma^{2}(\overline{\overline{S Y 103}})= \\
& \left\{.1557 *\left[\left(.89^{2} / 2\right)+\left(.01^{2} / 3\right)+\left(.1^{2} / 10\right)\right]\right\}+\left\{\left(.89^{2} * .017^{2}\right) / 2+\left(.01^{2} * .17^{2}\right) / 3+\left(.10^{2} * .051^{2}\right) / 10^{3}\right\}=.062
\end{aligned}
$$




\section{Appendix E}

\section{AN-107 Layer Density Consideration}




\section{Appendix E - AN-107 Layer Density Consideration}

Historically the buoyancy ratio in AN-107 was less than unity (Barker and Hedengren 2003). The current buoyancy ratio results for AN-107 indicate that the tank should display BDGRE behavior, although BDGRE behavior has not been indicated by tank data (Barker and Hedengren 2004). Thus, an apparent discrepancy exists.

The increase in the buoyancy ratio is attributable to alterations in the layer densities. (a) The liquid and sediment densities in AN-107 have historically been reported as 1.37 and $1.56 \mathrm{~g} / \mathrm{mL}$, respectively (Barker and Hedengren 2003), resulting in a buoyancy ratio of 0.53, with all other inputs set to those of Barker and Hedengren (2004). The density values from Barker and Hedengren (2004), 1.39 and $1.48 \mathrm{~g} / \mathrm{mL}$, result in a buoyancy ratio of 1.12 . The current reported density values, 1.43 and $1.48 \mathrm{~g} / \mathrm{mL}$ of the liquid and sediment, respectively, (b) result in a buoyancy ratio of 2.01, a result on par with that of AN-104 and AN-105.

TWINS(c) data for AN-107 was investigated in a manner similar to that described in the main report, Section 3.2.2. Median liquid and sediment density results are presented in Figure E.1.(d) Also included in the figure is the resulting buoyancy ratio with time [all parameters other than the layer densities are set to those listed in Barker and Hedengren (2004); the buoyancy ratio result for the 1996 data is averaged over the density values] and the caustic additions to the tank. As reflected in the data sources referenced above, the liquid density is apparently increasing with time. Plausible mechanisms for this to occur include evaporation, dissolution of solids caused by temperature increase, the caustic additions to the tank, and changes in the liquid chemical composition. These mechanisms are considered below. The apparent sediment density changes are also considered.

Investigation of the level trend shows that the conservative maximum cumulative loss attributable to evaporation from October 2000 to June 2003 (roughly the period of Figure E.1 excluding the 1996 data) is approximately 2 inches neglecting transfers and fluctuations (Figure E.2). Assuming that this cumulative loss is all attributable to evaporation of water, an initial liquid density of $1.37 \mathrm{~g} / \mathrm{mL}$ would be increased to approximately $1.373 \mathrm{~g} / \mathrm{mL}$. Thus, evaporation is ruled out as a significant cause of the apparent liquid density change of Figure E.1.

(a) The sediment depth has actually been slightly reduced, which lowers the buoyancy ratio.

(b) Best Basis Inventory accessed 4/15/05 at:

http://twins.pnl.gov/data/getLookupFields3.exe?table=bb_published.dbo.v_tank_waste_without_aggregate\&whatsn ew $=$ Best + Basis + Inventory

(c) TWINS, Tank Waste Information Network System, http://twins.pnl.gov/twins3/twins.htm.

(d) The bulk of the liquid reported liquid density results are specific gravity measurements (84\%) and are therefore expected to be accurate (see Section 3.2 of the main report). 


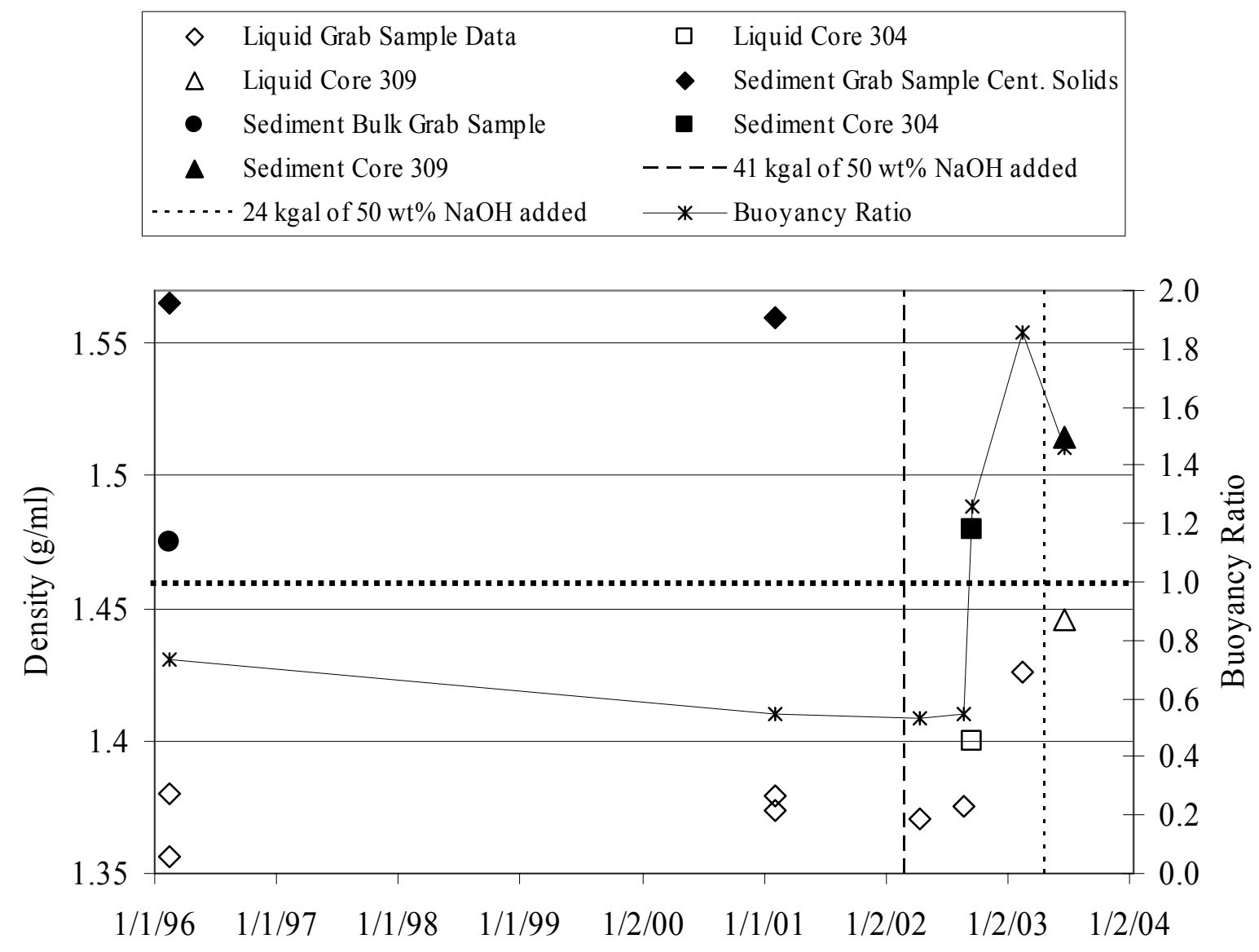

Figure E.1. Median Layer Densities and Buoyancy Ratio in AN-107 as a Function of Time

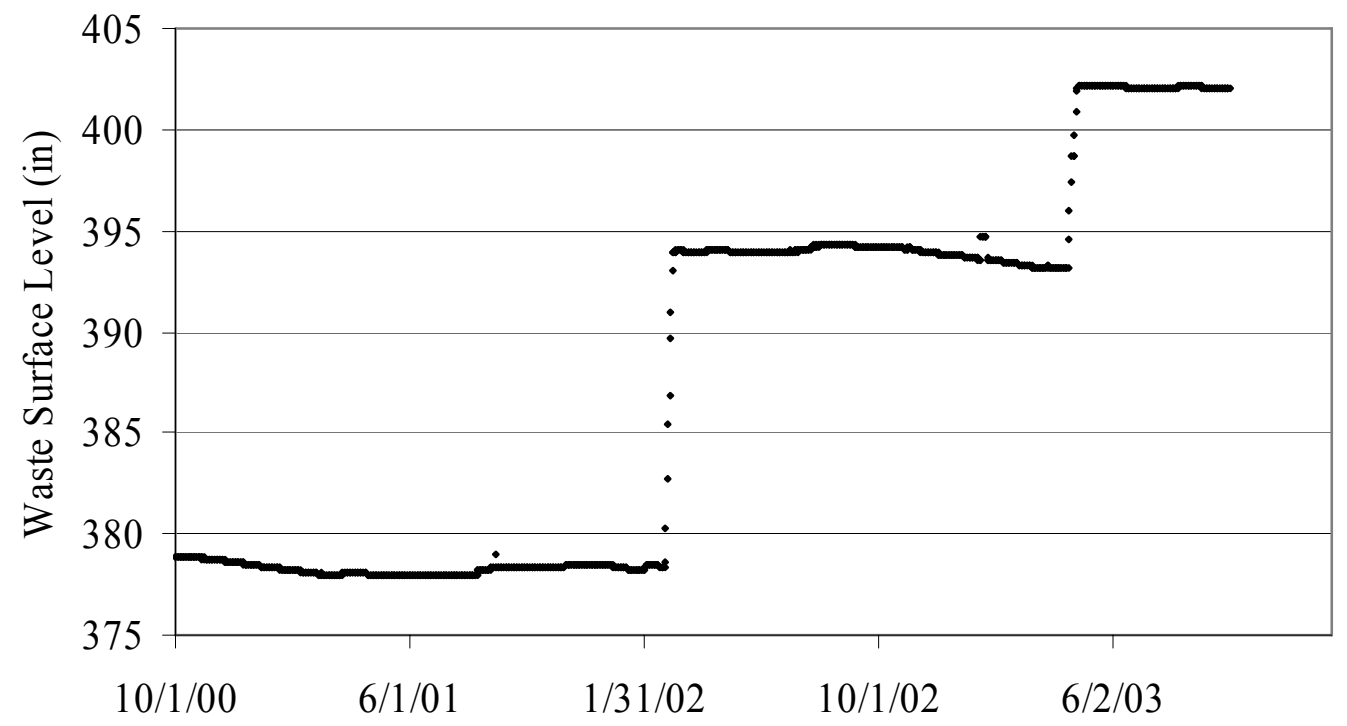

Figure E.2. Waste Surface Level in AN1-07 
Dissolution of solids resulting in the density increase is possibly ruled out by the aforementioned reported sediment level increase. However, the liquid layer temperature has increased by nominally $5^{\circ} \mathrm{F}\left(3^{\circ} \mathrm{C}\right)$. From Figure 3.3 in Section 3.2.2 of the main report, tank waste data indicate that the maximum density increase from $1.37 \mathrm{~g} / \mathrm{mL}$ from a $3^{\circ} \mathrm{C}$ temperature increase would be to $1.375 \mathrm{~g} / \mathrm{mL}$. This change, as with evaporation, is not sufficient to explain Figure E.1.

Two caustic additions of $50 \mathrm{wt} \% \mathrm{NaOH}$ were initiated in AN-107 on 2/28/02 and 4/23/03. A total of $65 \mathrm{kgal}$ of $50 \mathrm{wt} \% \mathrm{NaOH}$ was added. The specific gravity of $50 \mathrm{wt} \% \mathrm{NaOH}$ is nominally 1.52 (extrapolated from Weast 1975). The caustic addition should increase the liquid density in the tank. Assuming complete mixing, the resulting density when added to $1.37 \mathrm{~g} / \mathrm{mL}$ liquid is $1.381 \mathrm{~g} / \mathrm{mL}$. Thus caustic addition also does not provide an explanation of the apparent density increase of Figure E.1.

Consideration of the liquid chemical composition suggests that the liquid density change is real however, regardless of the prior arguments. (a) The liquid data summarized in Figure E.1 were sorted for those samples that also had chemical composition data. The grab sample data through 2002 all have a specific gravity of 1.39 or less, water fraction greater than $60 \mathrm{wt} \%$, and sodium molarity greater than $9.5 \mathrm{M} \mathrm{Na}$. Another sample, the drainable liquid from the 2002 core segment 304:01, also has a low specific gravity and high Na molarity but a lower water content, $52 \mathrm{wt} \%$. It is possible for a salt solution to have a sodium concentration of $10 \mathrm{M} \mathrm{Na}$ and a specific gravity of less than 1.4; for example, $10 \mathrm{M} \mathrm{NaOH}$ has a specific gravity of only 1.33. However, hydroxide concentration is not the key to the Specific gravity because this set of samples includes two samples with minimal hydroxide $(<0.01 \mathrm{M} \mathrm{OH})$ as well as the majority that have free $\mathrm{OH}$ of $0.5 \mathrm{M} \mathrm{OH}$ or greater.

Except for the one core sample mentioned above, all of the "interstitial liquid" samples from cores 304 (9/2002) and 309 (6/2003), as well as the 2/2003 supernatant grab samples, have Specific gravity of 1.39 or greater, sodium molarity less than $9.5 \mathrm{M} \mathrm{Na}$, and water fraction of $55 \mathrm{wt} \%$ or less. The free $\mathrm{OH}$ concentrations in this set range from $<0.01 \mathrm{M} \mathrm{OH}$ to $1.4 \mathrm{M} \mathrm{OH}$.

The change is not merely a matter of tank supernatant versus matrix interstitial liquid, because the supernatant grab samples taken in 2/2003 show the same change. No gross waste composition changes are immediately obvious. In conclusion, the change seems to be real, because there are two indicators besides specific gravity (water fraction and Na molarity).

Data analyses as described above for the liquid density cannot directly be made for the sediment. Instead, we compare the liquid-sediment density relation to other Hanford wastes. Expected Hanford waste behavior would indicate a higher sediment density. To explain, as described in Barker and Lechelt (2000) and Barker et al. (1999), the Hanford wastes may be classified in the following manner:

(a) Personal communication with LA Mahoney, PNNL. 
- $\quad$ Liquid waste (LIQ)

- $\quad$ Saltcake waste with $\geq 1 \mathrm{~m}$ liquid over solids (SC-LIQ)

- $\quad$ Saltcake waste with $<1 \mathrm{~m}$ liquid over solids (SC-NL)

- $\quad$ Sludge waste with $\geq 1 \mathrm{~m}$ liquid over solids (SL-LIQ)

- $\quad$ Sludge waste with $<1 \mathrm{~m}$ liquid over solids (SL-NL)

- $\quad$ Saltcake and sludge waste with $\geq 1 \mathrm{~m}$ liquid over solids (MX-LIQ)

- $\quad$ Saltcake and sludge waste with $<1 \mathrm{~m}$ liquid over solids (MX-NL).

AN-107 may be categorized as SC-LIQ. As may be seen from the available Hanford data in Figure E.3, wastes of this type with liquid densities of $1.4 \mathrm{~g} / \mathrm{mL}$ typically have sediment densities approaching $1.6 \mathrm{~g} / \mathrm{mL}$. AN-107 data from Core 309 appears on the plot as the lowest sediment density for this waste type. The bulk of Hanford wastes, regardless of liquid density value, have higher sediment densities. While certainly not conclusive, this observation may, in conjunction with the apparent lack of BDGREs in AN-107, suggest that the higher reported sediment density values may be more representative of tank conditions.

$\triangle \mathrm{SC}-\mathrm{LIQ} \times \mathrm{SC}-\mathrm{NL} * \mathrm{SL}-\mathrm{LIQ} O \mathrm{SL}-\mathrm{NL} \square \mathrm{MX}-\mathrm{NL}$

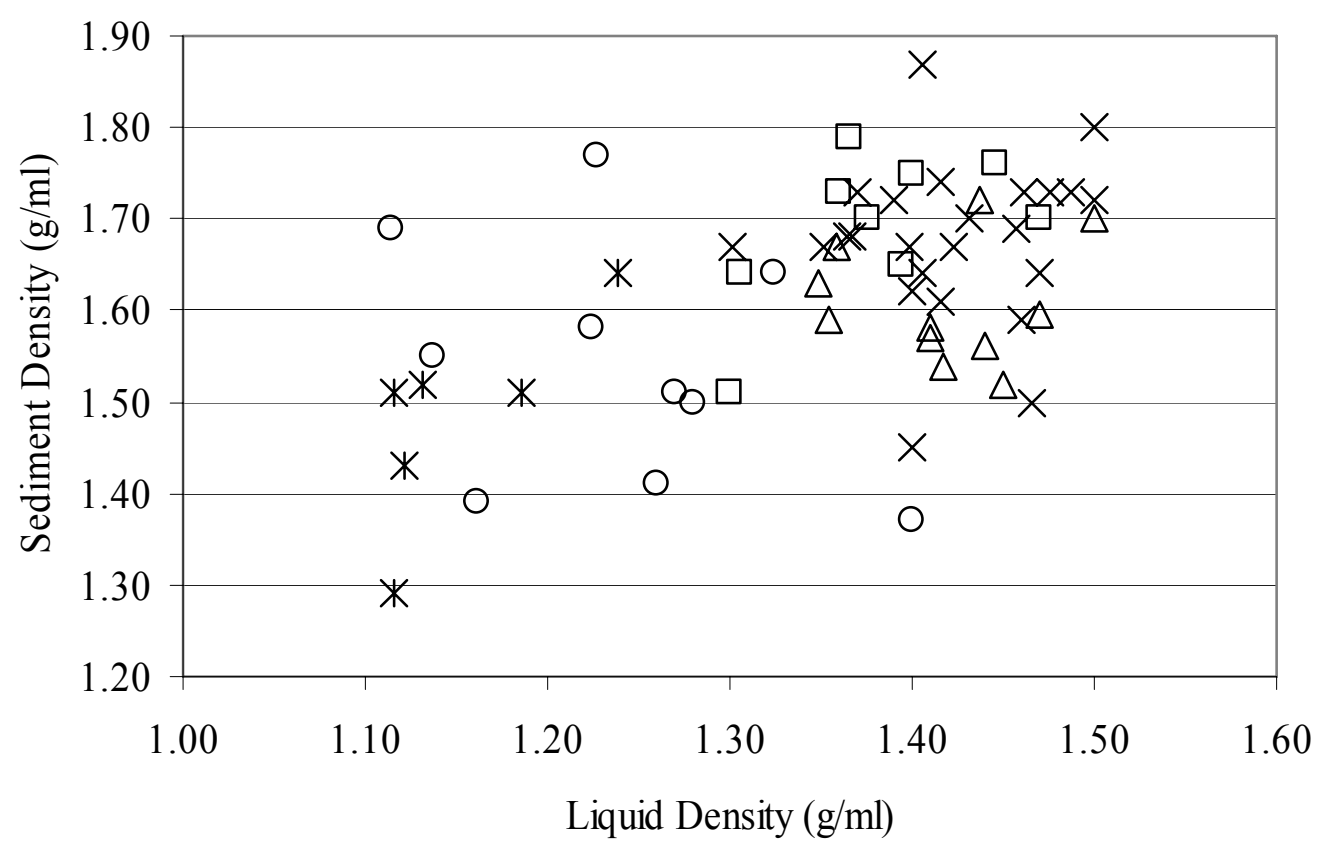

Figure E.3. Hanford Waste Liquid and Sediment Density 


\section{References}

Barker SA and DC Hedengren. 2003. Methodology and Calculations for the Assignment of Waste Groups for the Large Underground Waste Storage Tanks at the Hanford Site. RPP-10006 Rev. 1, CH2M HILL Hanford Group, Richland, Washington.

Barker SA and DC Hedengren. 2004. Methodology and Calculations for the Assignment of Waste Groups for the Large Underground Waste Storage Tanks at the Hanford Site. RPP-10006 Rev. 4, CH2M HILL Hanford Group, Richland, Washington.

Barker SA and AR Lechelt. 2000. Determination of Waste Groupings for Safety Analyses. RPP6171 Rev. 0, CH2M HILL Hanford Group, Inc., Richland, Washington.

Barker SA, WB Barton, DR Bratzel, M Epstein, PA Gauglitz, GD Johnson, SN Maruvada, CE Olson, ML Sauer, SE Slezak, CW Stewart, and J Young. 1999. Flammable Gas Safety Analysis Review. SNL-000198, Sandia National Laboratory, Albuquerque, New Mexico.

Weast RC, ed. 1975. Handbook of Chemistry and Physics. CRC Press, Inc. Cleveland, Ohio. 


\section{Appendix F}

Statistical Model Methodology 


\section{Appendix F - Statistical Model Methodology}

Distributions were created for the fraction of hydrogen in the retained gas, the liquid and sediment densities, and hydrogen generation rate based on the specifications derived from available data as discussed in Section 3.2. Ten thousand realizations for each variable were created in a statistical software, S-plus, and exported to an Excel workbook. These 10,000 realizations were then used to calculate 10,000 realizations of (BR-1) for each tank. This composed the Monte Carlo portion of the exercise.

The output BR-1 from the Monte Carlo portion of the exercise, along with its corresponding hydrogen fraction were then input back into S-plus, which randomly selected 30 representative data pairs from the population of 10,000. It is necessary to randomly choose a smaller number of outputs that represent the population in order to get a meaningful model. If all 10,000 data points are used, the model will arbitrarily show a low variability and high fit, due to the uncommonly large number of degrees of freedom available for the model (degrees of freedom are associated with the number of records in the data, in this case, 10,000). As the number of records becomes larger, the variability in the model and the data become smaller. Because these records were arbitrarily chosen to be 10,000 long, it would not be appropriate to form a model based on all the 10,000 (the resulting models would be falsely good). Instead, a more reasonable number of records, 30, was randomly chosen. According to the Central Limit Theorem, the mean of a distribution is asymptotically normally distributed when $\mathrm{n}$, the number of records, is large enough. "Large enough" has been proven to be at least 30 (Mendenhall et al. 1990). Therefore, 30 are enough to produce adequate models.

The final two variables needed for modeling are $(\mathrm{BR}-1)\left[\mathrm{H}_{2}\right]_{\text {gas }} / \mathrm{V}_{\mathrm{HS}}$ and $\left[\mathrm{H}_{2}\right]_{\text {peak }}$ (see Figure 3.1.2 in the main report). The 30 randomly selected data pairs were used in the appropriate calculations that produce these two needed variables for modeling. The two variables were extracted from an Excel spreadsheet (depending on which scenario was being performed: hot tanks, or mixed hot and cold tanks), and used to create appropriate models. The first model showed decent fit, but indicated a Lack of Fit was present. This means that the model may not be stable, as the variability associated with the model is larger than the variability inherent in the data. However, given that prediction intervals are the goal of this exercise, it may be prudent to allow this model to be used, as the larger variability will cause predictions to be over-estimated, hence, conservative. With this knowledge, it was decided to repeat the modeling several times to ensure that similar models would be constructed. Three to five models would have sufficed for our purposes, but it was decided to randomly select 30 new points from the 10,000 realizations 10 separate times, and construct 10 separate models to provide more assurance in our ultimate predictions. Therefore, 10 models were constructed for each scenario (hot tanks and mixed tanks). If the 10 models constructed varied dramatically, then it could be concluded that our models are not very stable, and our predictions not very reliable. There is nothing scientific about constructing 10 different models; another number could have been used. 
However, it is the expert opinion of the statistical scientist and domain engineers that 10 are more than sufficient to meet our needs.

The model statistics for the linear models of the hot tanks are shown in Table F.1, with the "best" model shown in bold. A good model will have a high p-value for Lack of Fit, a high value for R-square (the correlation), and a low value for RMSE (Residual Mean Squared Error). This best model is representative of what the full data set would have constructed, if real data had been used. As can be seen in Table F.1, Model 9 has the best R-square, Lack of Fit, and RMSE.

Table F.1. Linear Models for Hot Tanks

\begin{tabular}{|c|c|c|c|}
\hline Model & R-square & Lack of Fit p-value & RMSE \\
\hline 1 & 0.7 & 0.0353 & 11300 \\
\hline 2 & 0.71 & 0.0543 & 11146 \\
\hline 3 & 0.7 & 0.0002 & 11293 \\
\hline 4 & 0.66 & 0.0545 & 12073 \\
\hline 5 & 0.65 & 0.2225 & 12155 \\
\hline 6 & 0.7 & 0.0005 & 11354 \\
\hline 7 & 0.7 & 0.1909 & 11197 \\
\hline 8 & 0.7 & 0.0001 & 11354 \\
\hline 9 & 0.75 & 0.5132 & 10335 \\
\hline 10 & 0.66 & 0.0299 & 12022 \\
\hline
\end{tabular}

The model statistics for the quadratic models of the hot tanks are shown in Table F.2, with the "best" model shown in bold. Model 9 has the best R-square, best lack of fit, and best RMSE.

Table F.2. Quadratic Models for Hot Tanks

\begin{tabular}{|c|c|c|c|}
\hline Model & R-square & Lack of Fit p-value & RMSE \\
\hline 1 & 0.7 & 0.0347 & 11327.6 \\
\hline 2 & 0.71 & 0.053 & 11182.42 \\
\hline 3 & 0.71 & 0.0002 & 11267.06 \\
\hline 4 & 0.66 & 0.0536 & 12114 \\
\hline 5 & 0.69 & 0.2798 & 11620.39 \\
\hline 6 & 0.7 & 0.0005 & 11335.9 \\
\hline 7 & 0.72 & 0.2008 & 11087.26 \\
\hline 8 & 0.7 & 0.0001 & 11390.96 \\
\hline 9 & 0.76 & 0.5458 & 10126.36 \\
\hline 10 & 0.66 & 0.0294 & 12060.71 \\
\hline
\end{tabular}


The model statistics for the linear models of the mixed tanks are shown in Table F.3, with the "best" model shown in bold. Note that the LOF p-values for all of the models, except model 5, reject for lack of fit, meaning those models have more variability associated with the model itself than in the data. Model 5 barely makes the cut (the cutoff is .05). The "best" model is the one with the best overall results. Model 9 has the highest R-square and lowest RMSE, while Model 5 has the worst R-square and worst RMSE. Therefore, Model 9 was chosen as the "best," because the improvement in lack of fit for Model 5 resulted in too much of a loss in the other two categories.

Table F.3. Linear Models for Mixed Tanks

\begin{tabular}{|c|c|c|c|}
\hline Model & R-square & Lack of Fit p-value & RMSE \\
\hline 1 & 0.67 & 0.0001 & 10094.97 \\
\hline 2 & 0.67 & 0.0001 & 10148.42 \\
\hline 3 & 0.66 & 0.0001 & 10257.97 \\
\hline 4 & 0.64 & 0.0001 & 10538.87 \\
\hline 5 & 0.61 & 0.0994 & 10963 \\
\hline 6 & 0.67 & 0.0001 & 10117.85 \\
\hline 7 & 0.67 & 0.0001 & 10052.17 \\
\hline 8 & 0.65 & 0.0001 & 10481.49 \\
\hline 9 & 0.71 & 0.0001 & 9558.39 \\
\hline 10 & 0.63 & 0.0001 & 10784.1 \\
\hline
\end{tabular}

The model statistics for the quadratic models of the mixed tanks are shown in Table F.4, with the "best" model shown in bold. Again, Model 9 had the best R-square and RMSE, but not the best lack of fit.

Table F.4. Quadratic Models for Mixed Tanks

\begin{tabular}{|c|c|c|c|}
\hline Model & R-square & Lack of Fit p-value & RMSE \\
\hline 1 & 0.69 & 0.0001 & 9790.469 \\
\hline 2 & 0.69 & 0.0001 & 9884.015 \\
\hline 3 & 0.69 & 0.0001 & 9778.48 \\
\hline 4 & 0.65 & 0.0001 & 10369.25 \\
\hline 5 & 0.69 & 0.3338 & 9836.795 \\
\hline 6 & 0.7 & 0.0001 & 9672.479 \\
\hline 7 & 0.71 & 0.0005 & 9479.057 \\
\hline 8 & 0.67 & 0.0001 & 10162.34 \\
\hline 9 & 0.76 & 0.0031 & 8693.418 \\
\hline 10 & 0.64 & 0.0001 & 10591.22 \\
\hline
\end{tabular}


Once a model is chosen to use for predictions, a 95\% prediction interval is calculated (a 95\% confidence interval on the prediction) based on the chosen model constructed from the randomly selected 30 data points. The prediction interval is calculated as

$$
\mathrm{Y} \pm 1.976122 \sqrt{\mathrm{SE}_{\text {pred }}^{2}+\mathrm{SE}_{\text {ind }}^{2}}
$$

where

$\mathrm{Y}=$ the predicted value from the model

$1.976122=$ the $\mathrm{t}$ statistic associated with $\mathrm{t}$-distribution(a) with $\mathrm{n}-2=28$ degrees of freedom

$\mathrm{SE}_{\text {pred }}^{2} \quad=$ the standard error of the prediction

$\mathrm{SE}_{\text {ind }}^{2} \quad=$ the standard error of the individual.

The standard error of the prediction accounts for all the uncertainty in the parameter estimates. It is calculated by using the covariance matrix associated with the parameter estimates, and a composition vector of the partial derivatives of the model with respect to each parameter.

The standard error of the individual accounts for the uncertainty in predicting the individual Y's. It is calculated by using the covariance matrix associated with the parameter estimates, the composition of the partial derivatives of the model with respect to each parameter, and the estimate of the error variance (MSE).

\section{Reference}

Mendenhall W, D Wackerly, and R Scheaffer. 1990. Mathematical Statistics with Applications. PWS-KENT Publishing Company, Boston.

(a) The t-distribution is, asymptotically, a normal distribution; 1.96, the z-score associated with the normal distribution, could be used instead, as a good approximation. 
PNNL-15238

\section{Distribution}

No. of

Copies

7 CH2MHILL

SA Barker

DC Hedengren

NW Kirch

LJ Kripps

JE Meacham

Correspondence Control

TCSRC
No. of

Copies

11 Pacific Northwest National Laboratory

S5-08

S5-08

$\mathrm{R} 2-58$

S7-90

R2-58

H6-08

R1-10
K6-50

SQ Bennett

K9-69

TM Brouns

P7-27

MS Fountain

K6-08

SA Hartley

K7-15

LA Mahoney

K7-15

K7-15

TE Michener

K7-15

CW Stewart

K7-15

BE Wells

P8-55 